\title{
USING THE TECHNOLOGY ACCEPTANCE MODEL TO DETERMINE STUDENT PERCEPTION OF ELEARNING READINESS IN KAZAKHSTAN
}

\section{by}

Anthony Ray Hetrick

\author{
A dissertation \\ submitted in partial fulfillment \\ of the requirements for the degree of \\ Doctor of Education in Educational Technology \\ Boise State University
}

May 2019 
Anthony Ray Hetrick

\section{SOME RIGHTS RESERVED}

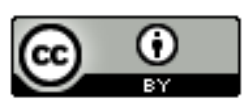

This work is licensed under a Creative

Commons Attribution 4.0 International

License 


\title{
BOISE STATE UNIVERSITY GRADUATE COLLEGE
}

\section{DEFENSE COMMITTEE AND FINAL READING APPROVALS}

\author{
of the Dissertation submitted by
}

Anthony Ray Hetrick

Dissertation Title: Using the Technology Acceptance Model to Determine Student Perception of eLearning Readiness in Kazakhstan

Date of Final Oral Examination: $\quad 07$ December 2018

The following individuals read and discussed the dissertation submitted by student Anthony Ray Hetrick, and they evaluated his presentation and response to questions during the final oral examination. They found that the student passed the final oral examination.

Ross Perkins, Ph.D. Chair, Supervisory Committee

Jui-long Hung, Ed.D. Member, Supervisory Committee

Brett E. Shelton, Ph.D. Member, Supervisory Committee

The final reading approval of the dissertation was granted by Ross Perkins, Ph.D., Chair of the Supervisory Committee. The dissertation was approved by the Graduate College. 


\section{DEDICATION}

I dedicate my dissertation to my loving and supportive wife, Janat, who inspired me to pursue this degree and then encouraged me along the way. 


\section{ACKNOWLEDGEMENTS}

Thank you, Dr. Perkins, for serving as my chair, providing inspiration, support, and guidance as you coached me through this project. You found time to support me at the expense of your schedule. I am deeply appreciative of your sacrifice.

Thank you, Dr. Hung, for the extra support, time, and expertise you gave me throughout this project. I was pursuing a different type of project until I took your Advanced Stats course, which then gave life to this study.

Thank you, Dr. Shelton, for serving on my committee, providing pertinent feedback, and pointing out deficiencies of the study. I appreciated your comments as they fine-tuned and clarified this study.

Thank you, Ms. Saule Kumargazhanova and Ms. Madina Yussubaliyeva, for making it possible to conduct this research at your university. Your support for this project, coordinating the students and the ICT class, and navigating the university's bureaucracy on my behalf turned this project from an idea to a reality. Again, thank you. 


\begin{abstract}
Researchers widely accept the technology acceptance model (TAM) to determine behavioral intention that leads to actual technology use. However, researchers are advised to exercise caution when applying TAM to different cultural contexts. This study used TAM to assess the readiness of students to engage in elearning in Kazakhstan, which is classified as a developing nation. This project then compared the results of the TAM analysis of student perceptions of a learning management system (LMS) to elearning studies in developed countries to ascertain if the determinants are the same. This study determined that TAM was unpredictable, and that perceived ease of use's significant impact on perceived usefulness was the only similarity to studies in developed countries when using the TAM factors of perceived usefulness (PU), perceived ease of use (PEOU), attitude toward using (ATT), and behavioral intention to use (BI).

Contrastingly, TAM functioned with consistency when removing the ATT construct, even though the results were different from developed countries because PEOU did not significantly influence BI.

The scientific merit of this research determined that TAM is unreliable in Kazakhstan and produced different results from developed countries. The global impact of this study provides researchers in other developing countries with data on how the combined cultural dimensions of high power distance, high masculinity, and high collectivism affected TAM.
\end{abstract}




\section{TABLE OF CONTENTS}

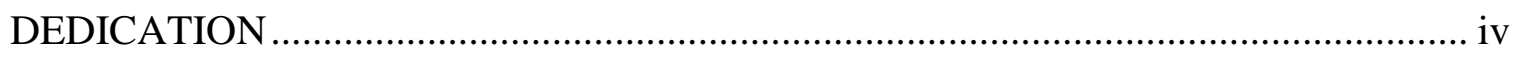

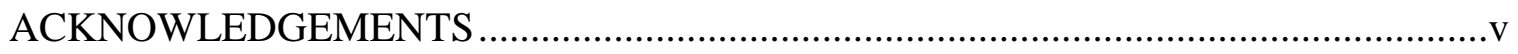

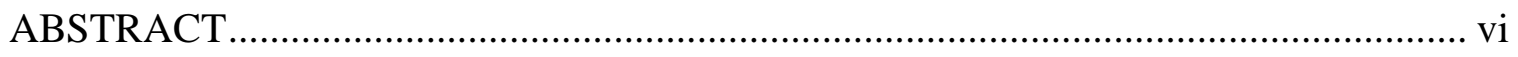

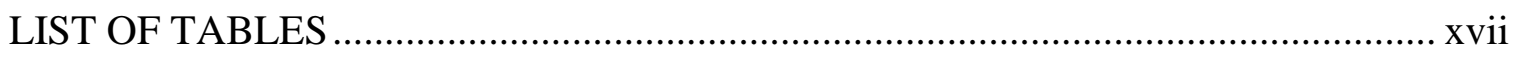

Appendix B Tables .................................................................................. xviii

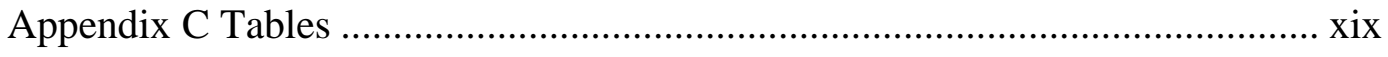

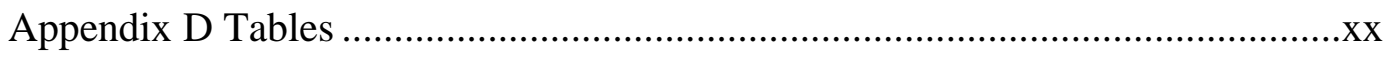

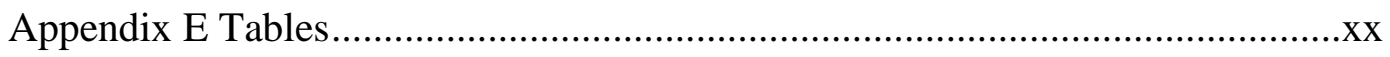

Appendix F Tables ………………………............................................... xxi

Appendix G Tables ................................................................................. xxii

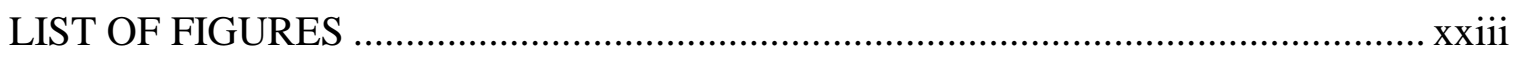

LIST OF ABBREVIATIONS ....................................................................... xxiv

Abbreviations for TAM Factors................................................................. xxiv

Abbreviations for Survey Codes ................................................................... xxiv

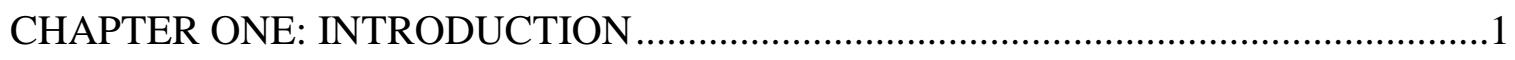

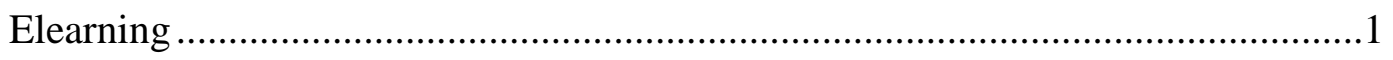

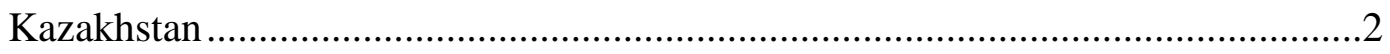

Using TAM to Determine Elearning Readiness ......................................................

Purpose of the Study …………………………….......................................... 
Expected TAM Results ......................................................................6

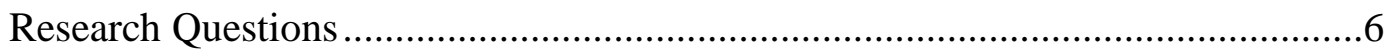

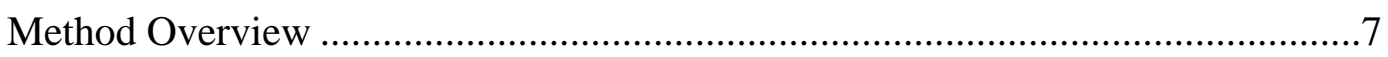

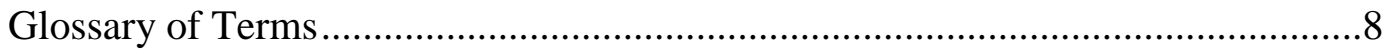

CHAPTER TWO: THE KAZAKHSTAN CONTEXT ……...........................................10

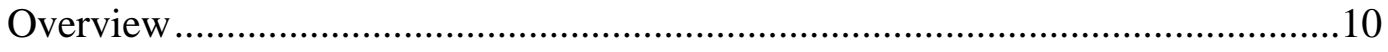

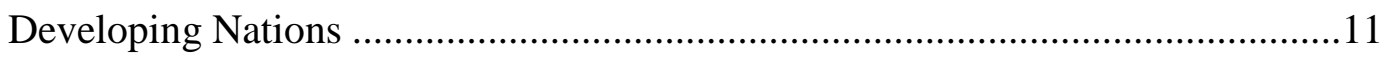

Elearning in Developing Countries..................................................................12

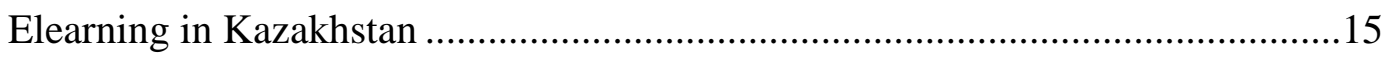

Hofstede's Profile of Kazakhstan ....................................................................17

Problems with Existing Literature ……………………..........................17

Results from Empirical Studies ..........................................................19

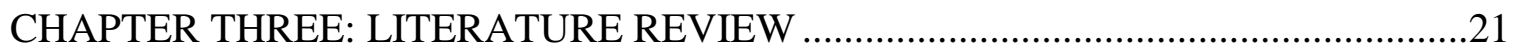

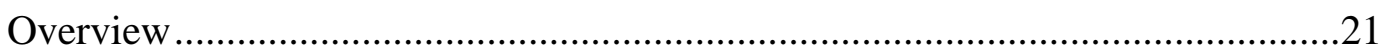

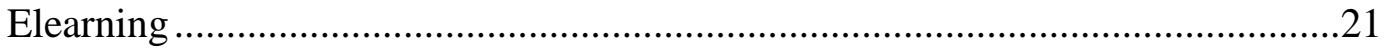

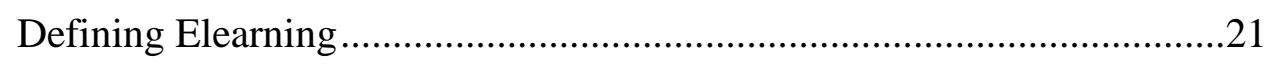

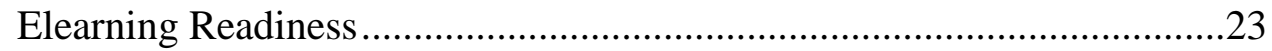

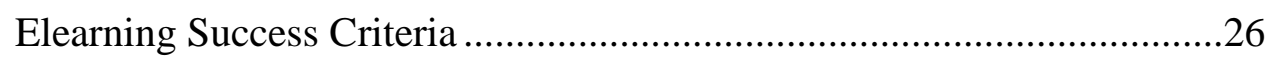

Technology Acceptance Model ............................................................................2

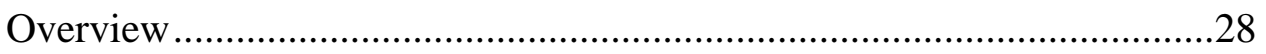

Theoretical Framework ………………………………........................29

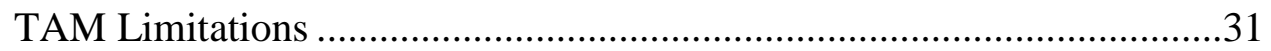

TAM in Elearning Contexts........................................................................43 


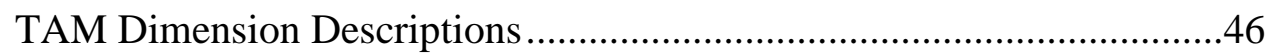

TAM Results from Different Countries .....................................................47

Learning Management System ……………………....................................51

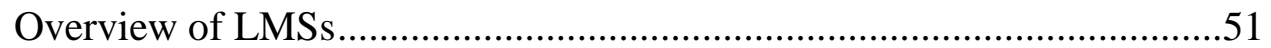

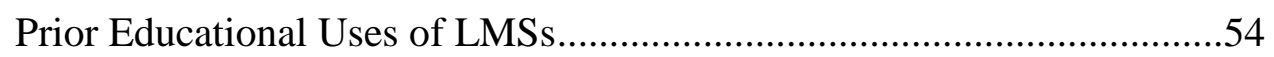

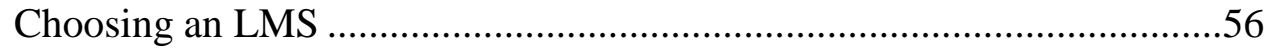

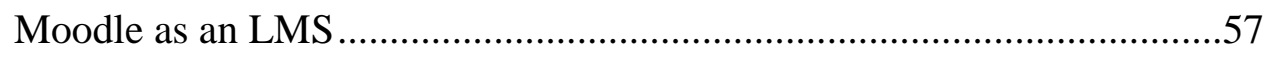

Data Analytics in Learning Management Systems ..............................................59

Data Mining and Moodle …………………………………………........61

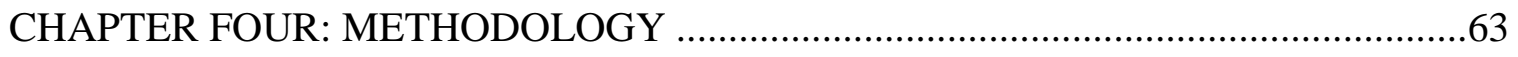

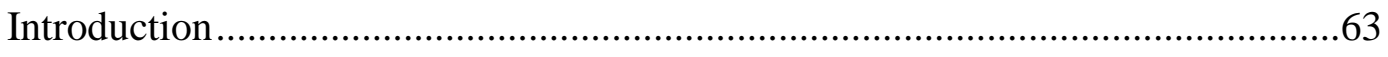

Statement of the Problem and Research Questions ...............................................64

Research Methodology ……………………………...................................64

Principal Component Analysis (PCA) ……………………………….....65

Path Analysis ......................................................................................66

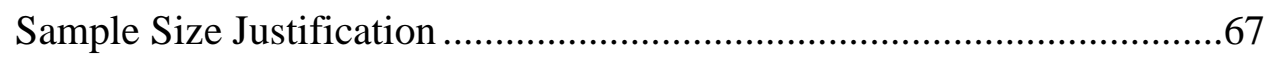

Learning Management System ……………….........................................67

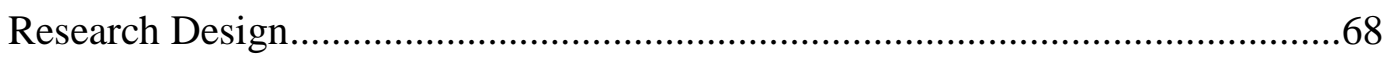

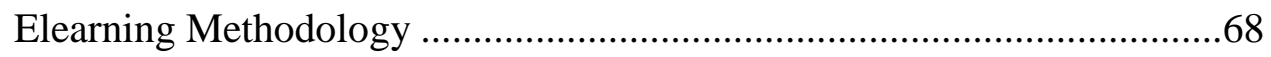

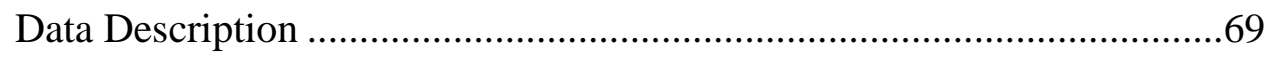

Principal Component Analysis ………………………………………......71

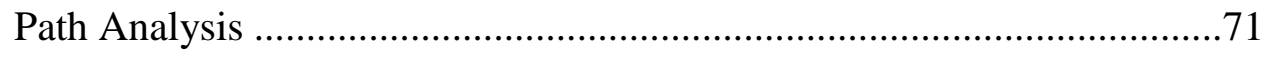

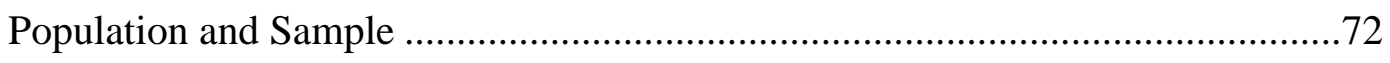




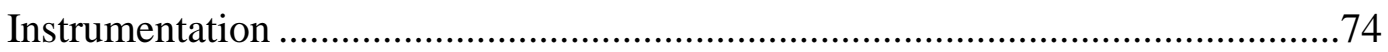

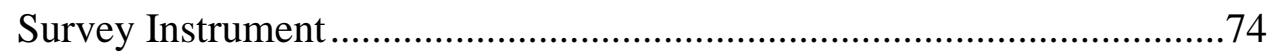

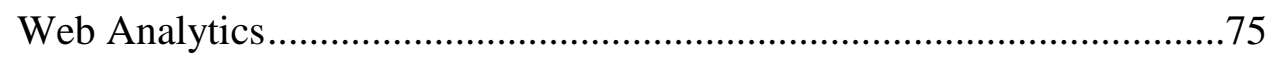

Data Management and Collection..................................................................76

Data Analysis and Procedures ………………...................................................

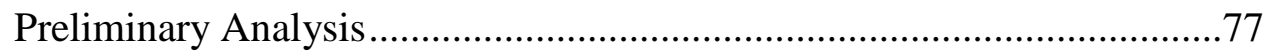

Principal Component Analysis ....................................................................77

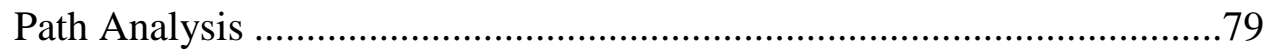

Analyzing External Factors..................................................................8

Answering Research Questions ..............................................................81

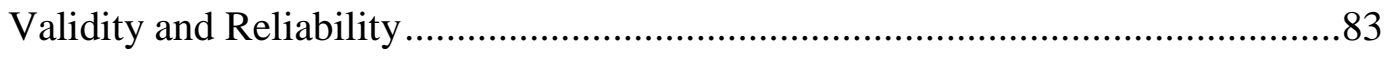

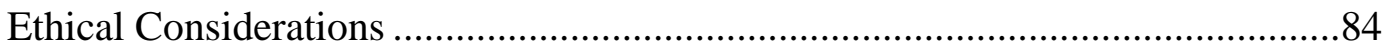

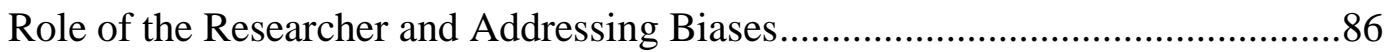

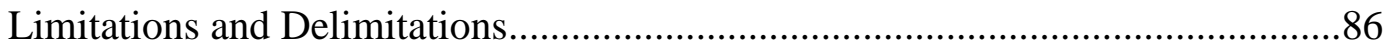

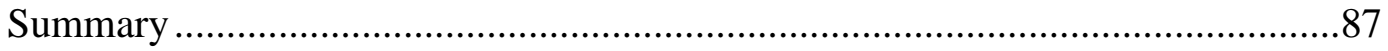

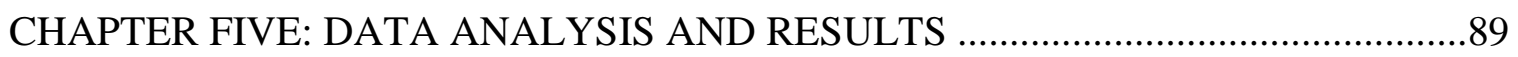

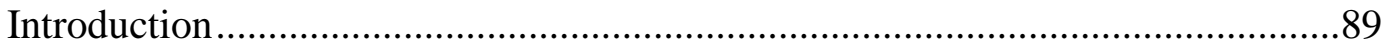

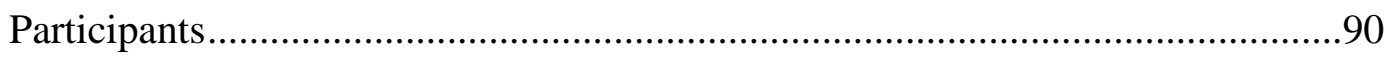

Gender and Language (DEM) .............................................................91

Technical Access and Ease of Use (TEC) ...............................................93

Previous Elearning Experience (PEX) ………………………….............96

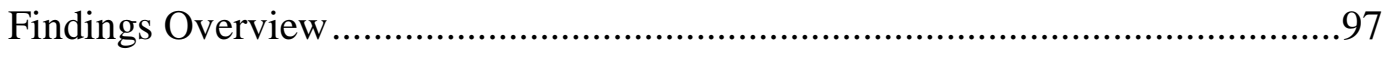

Research Question 1 Results....................................................................97 
Research Question 2 Results.........................................................98

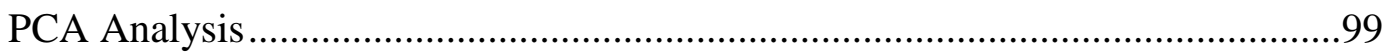

Survey I PCA Results .............................................................100

Survey II PCA Results .................................................................101

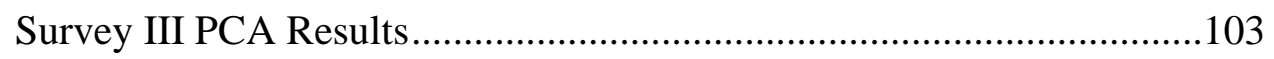

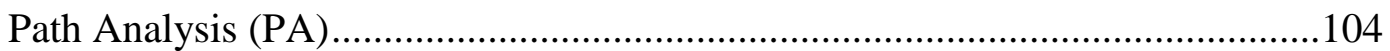

Survey I PA Results .....................................................................10

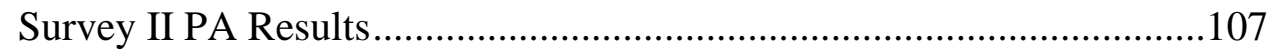

Survey III PA Results .............................................................110

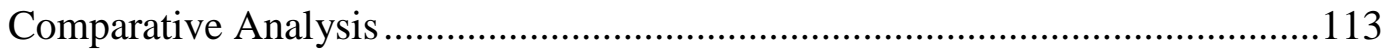

Research Question 1 Analysis ......................................................113

Research Question 2 Analysis ....................................................114

Learning Management System Objective Data ..............................................119

English-Speaking Course Analytics .............................................120

Guest-Access Course Analytics .....................................................123

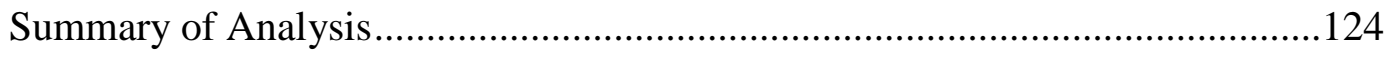

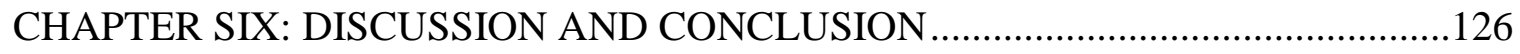

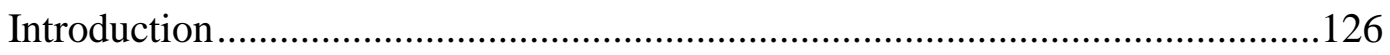

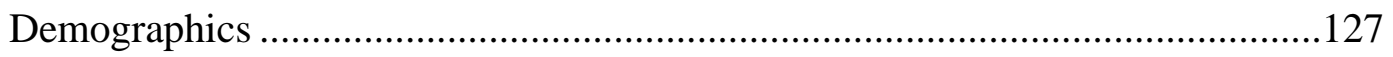

Sample Discrepancies ............................................................127

Evaluating TAM Factors in Kazakhstan....................................................128

Summary of TAM Effects ............................................................128

Evaluating TAM Expectations from Developed Countries ....................129 
Examining Cultural Data

Research Question 1: Does PU have a stronger influence than PEOU on ATT in Kazakhstan?

Research Question 2: How do the significant factors of PU, PEOU, ATT, and BI in Kazakhstan compare to the significant factors in developed countries?

Limitations and Further Research ............................................................ 138

Culture Discussion and Implications ............................................................141

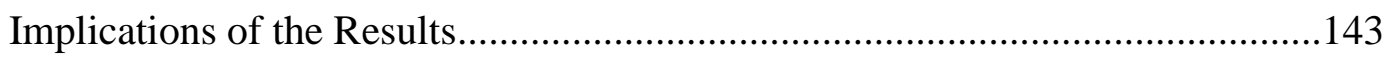

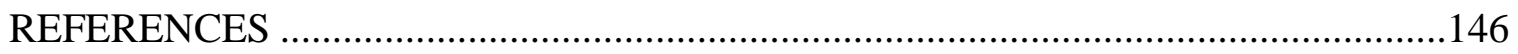

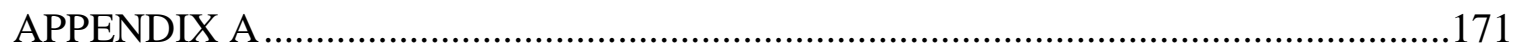

Referenced TAM Questions ..................................................................171

Perceived Usefulness of elearning (PU) ..........................................172

Perceived Ease of Use of elearning (PEOU) .....................................172

Attitude Toward Using elearning (ATT) .........................................172

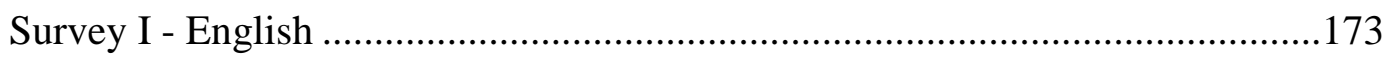

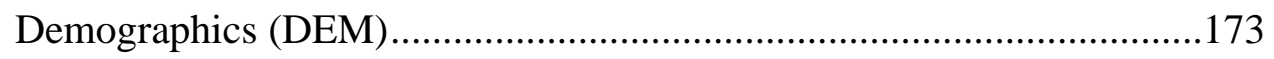

Previous Elearning Experience (PEX) ..........................................173

Perceived Usefulness of elearning (PU) ..........................................174

Perceived Ease of Use of elearning (PEOU) .....................................174

Attitude Toward Using elearning (ATT) .........................................174

Behavioral Intention towards using elearning (BI) ............................175

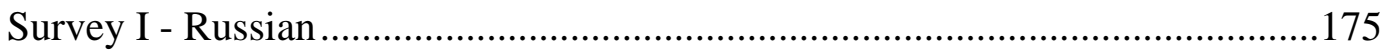

Демографические данные (DEM) ..............................................175

Предыдущий опыт интернет обучения (PEX)..................................176 
Оцениваемая практичность интернет обучения (PU)

Оцениваемая простота использования системы интернет обучения

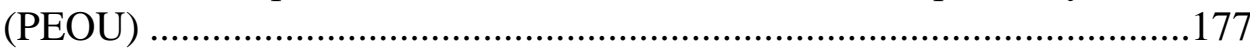

Отношение к использованию интернет обучения (АТT).................177

Намерение использовать электронное обучение (BI) .....................177

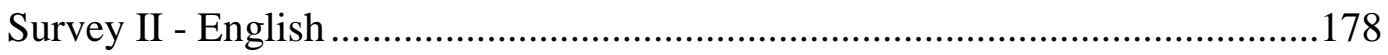

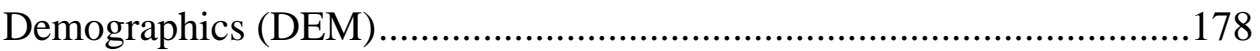

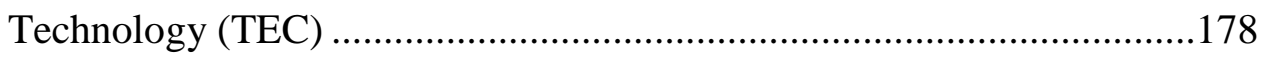

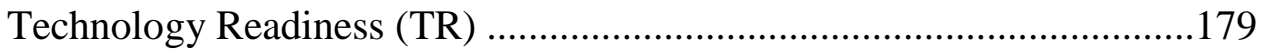

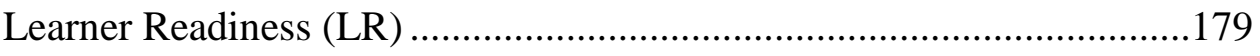

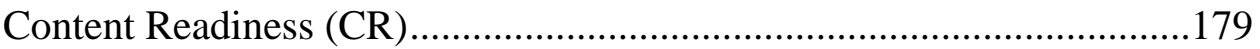

Previous Elearning Experience (PEX) ............................................180

Perceived Usefulness of elearning (PU) ........................................180

Perceived Ease of Use of elearning (PEOU) ......................................180

Attitude Toward Using elearning (ATT) ..........................................181

Behavioral Intention towards using elearning (BI) ............................181

External Factors (only PU) ........................................................181

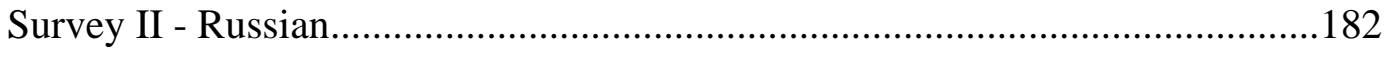

Демографические данные (DEM) ................................................182

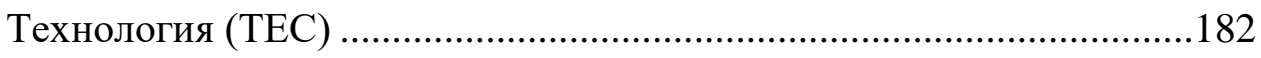

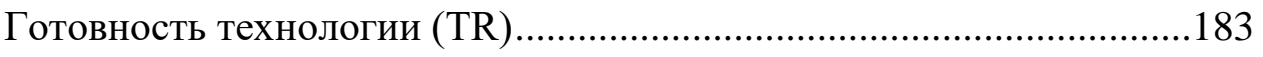

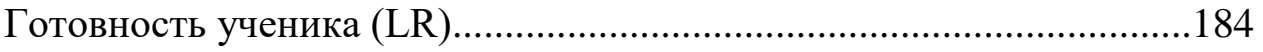

Содержание готовности (CR) ................................................... 184

Предыдущий опыт интернет обучения (PEX)................................184 
Оцениваемая практичность интернет обучения (PU)

Оцениваемая простота использования системы интернет обучения (PEOU) .................................................................................. 185

Отношение к использованию интернет обучения (АТТ).................185

Намерение использовать электронное обучение (BI) .......................186

Внешние факторы (только оцениваемая практичность электронного

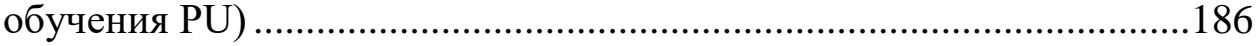

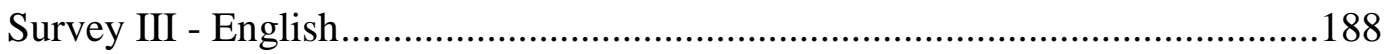

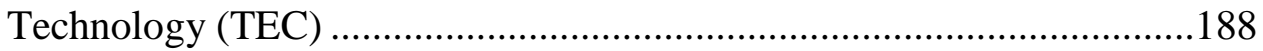

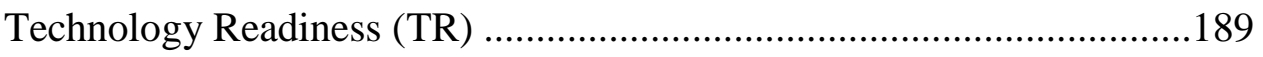

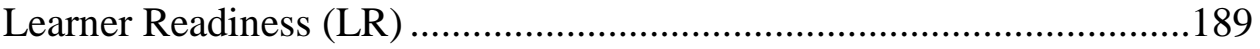

Content Readiness (CR) ..............................................................189

Previous Elearning Experience $(\mathrm{PEX})$............................................189

Perceived Usefulness of elearning (PU) ...........................................190

Perceived Ease of Use of elearning (PEOU) .....................................190

Attitude Toward Using elearning (ATT) ...........................................190

Behavioral Intention towards using elearning (BI)............................190

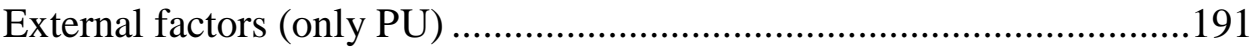

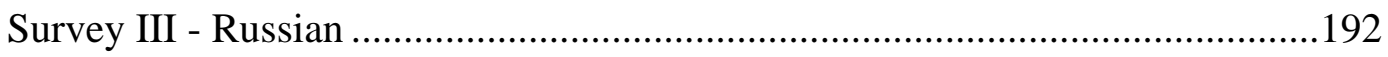

Демографические данные (DEM) ................................................192

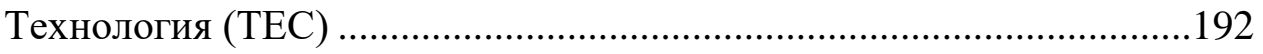

Готовность технологии (TR) .........................................................193

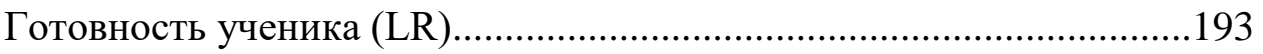

Содержание готовности (CR) .....................................................193 
Предыдущий опыт интернет обучения (PEX).

Оцениваемая практичность интернет обучения (PU)

Оцениваемая простота использования системы интернет обучения (PEOU) 195

Отношение к использованию интернет обучения (АТТ). 195

Намерение использовать электронное обучение (BI) 195

Внешние факторы (только оцениваемая практичность электронного обучения PU) 195

APPENDIX B .198

PCA Analysis Tables: Survey I 198

Original TAM: Survey I PCA. .199

Simplified TAM: Survey I PCA .204

APPENDIX C .208

PCA Analysis Tables: Survey II .... .208

Original TAM: Survey II PCA .209

Simplified TAM: Survey II PCA .212

APPENDIX D .215

PCA Analysis Tables: Survey III..... .215

Original TAM: Survey III PCA .216

Simplified TAM: Survey III PCA. .221

APPENDIX E .224

Path Analysis Tables: Survey I .224

Original TAM: Survey I Path Analysis .225

Simplified TAM: Survey I Path Analysis . .228 


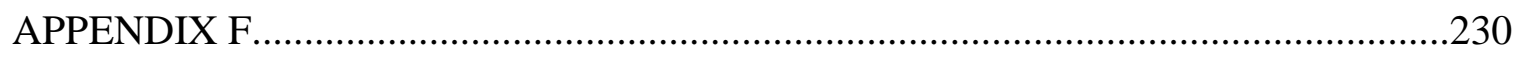

Path Analysis Tables: Survey II................................................................2230

Original TAM: Survey II Path Analysis ...........................................231

Simplified TAM: Survey II Path Analysis........................................234

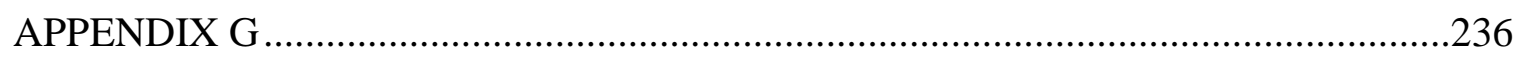

Path Analysis Tables: Survey III ..............................................................236

Original TAM: Survey III Path Analysis ...........................................237

Simplified TAM: Survey III Path Analysis ..........................................240 


\section{LIST OF TABLES}

Table 1: $\quad$ Expected Significant Paths for Kazakhstan (McCoy et al., 2007)............. 6

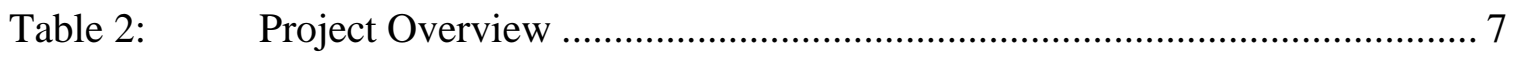

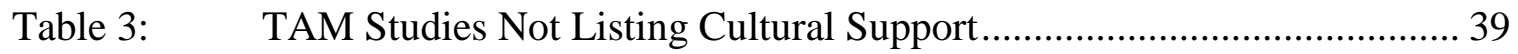

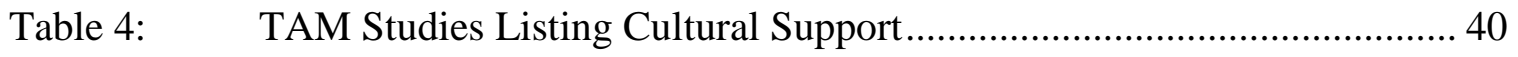

Table 5: $\quad$ Expected Significant TAM Paths (McCoy et al., 2007) .......................... 41

Table 6: $\quad$ Direct Effects using Educational Studies (Original TAM) ...................... 48

Table 7: $\quad$ Direct Effects using non-Educational Studies (Original TAM) .............. 49

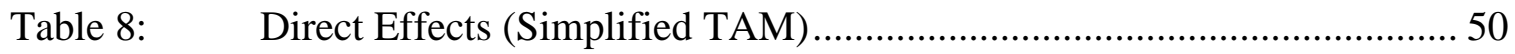

Table 9: $\quad$ Alignment of Research Questions to Data Analysis .............................. 82

Table 10: Demographics (General) DEM1-DEM4 ….......................................... 92

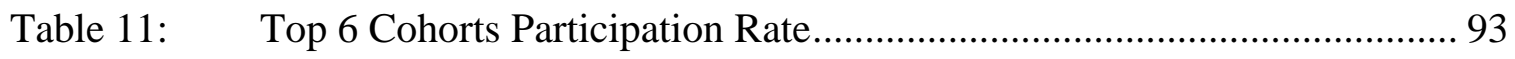

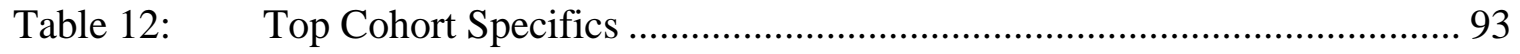

Table 13: $\quad$ Demographics (Technical Access and Ease of Use) TEC1-8................. 95

Table 14: $\quad$ Previous Elearning Experience (PEX1-2) ......................................... 96

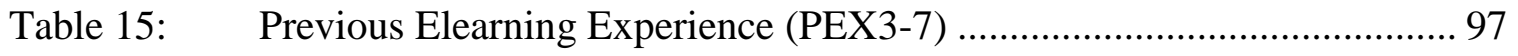

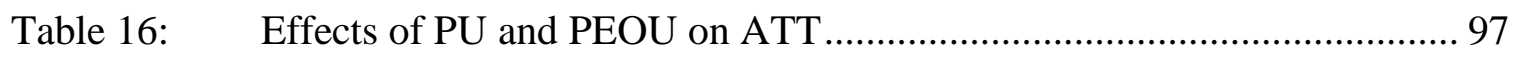

Table 17: $\quad$ Results in Kazakhstan (Original TAM) ............................................ 98

Table 18: $\quad$ Results in Kazakhstan (Simplified TAM).......................................... 99

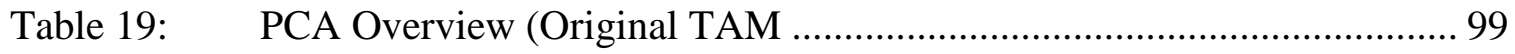


Table 20: $\quad$ PCA Overview (Simplified TAM) ...................................................... 100

Table 21: $\quad$ Survey I Path Analysis Results (Original TAM) …………………........ 105

Table 22: $\quad$ Survey I Path Analysis Results (Simplified TAM).................................. 105

Table 23: $\quad$ Survey II Path Analysis Results (Original TAM) .................................... 108

Table 24: $\quad$ Survey II Path Analysis Results (Simplified TAM) ................................ 108

Table 25: $\quad$ Survey III Path Analysis Results (Original TAM) .................................. 111

Table 26: $\quad$ Survey III Path Analysis Results (Simplified TAM) ................................ 111

Table 27: $\quad$ Number of Significant Paths (Original TAM) ........................................ 115

Table 28: $\quad$ Studies with non-Significant Paths (Original TAM) .............................. 116

Table 29: $\quad$ Number of Significant and Influential Paths (Simplified TAM) ............ 116

Table 30: $\quad$ Studies with non-Significant Paths (Simplified TAM)........................... 117

Table 31: Comparison of Significant Values (Original TAM) .............................. 118

Table 32: $\quad$ Comparison of Significant Values (Simplified TAM)............................ 119

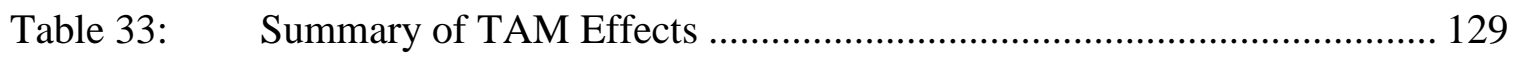

Table 34: $\quad$ Comparison of Expected TAM Results from Developed Countries ...... 131

Table 35: $\quad$ Expected Significant Paths for Kazakhstan (McCoy et al., 2007).......... 133

Table 36: $\quad$ Effects of PU and PEOU on ATT ......................................................... 134

Table 37: Comparison of Paths (Original TAM) ……………………………..... 137

Table 38: $\quad$ Comparison of Paths (Simplified TAM) ................................................ 138

\section{Appendix B Tables}

Table B1: $\quad$ Survey I, Original TAM PCA: Correlation Matrix a ……........................ 199

Table B2: $\quad$ Survey I, Original TAM PCA: Anti-image Correlation ........................... 200

Table B3: $\quad$ Survey I, Original TAM PCA: KMO and Bartlett's Test ....................... 200 
Table B4: $\quad$ Survey I, Original TAM PCA: Communalities .................................. 201

Table B5: $\quad$ Survey I, Original TAM PCA: Total Variance Explained...................... 202

Table B6: $\quad$ Survey I, Original TAM PCA: Structure Matrix ................................. 203

Table B7: $\quad$ Survey I, Original TAM PCA: Component Correlation Matrix ............. 203

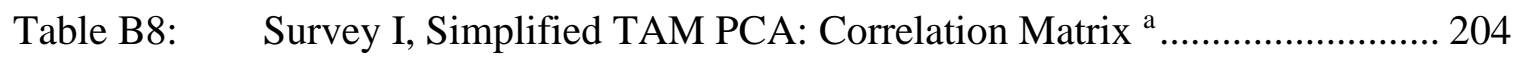

Table B9: $\quad$ Survey I, Simplified TAM PCA: KMO and Bartlett's Test ................... 204

Table B10: $\quad$ Survey I, Simplified TAM PCA: Communalities ................................. 205

Table B11: $\quad$ Survey I, Simplified TAM PCA: Total Variance Explained ................... 206

Table B12: $\quad$ Survey I, Simplified TAM PCA: Structure Matrix............................... 207

Table B13: $\quad$ Survey I, Simplified TAM PCA: Component Correlation Matrix ......... 207

\section{Appendix C Tables}

Table C1: $\quad$ Survey II, Original TAM PCA: Correlation Matrix a ........................... 209

Table C2: $\quad$ Survey II, Original TAM PCA: Anti-image Matrices .......................... 209

Table C3: $\quad$ Survey II, Original TAM PCA: KMO and Bartlett's Test .................... 210

Table C4: $\quad$ Survey II, Original TAM PCA: Communalities ….............................. 210

Table C5: $\quad$ Survey II, Original TAM PCA: Total Variance Explained .................... 211

Table C6: $\quad$ Survey II, Original TAM PCA: Structure Matrix ................................ 211

Table C7: $\quad$ Survey II, Original TAM PCA: Component Correlation Matrix............ 212

Table C8: $\quad$ Survey II, Simplified TAM PCA: Correlation Matrix ${ }^{\mathrm{a}}$.......................... 212

Table C9: $\quad$ Survey II, Simplified TAM PCA: KMO and Bartlett's Test.................. 212

Table C10: $\quad$ Survey II, Simplified TAM PCA: Communalities .............................. 213

Table C11: $\quad$ Survey II, Simplified TAM PCA: Total Variance Explained................. 213

Table C12: $\quad$ Survey II, Simplified TAM PCA: Structure Matrix ............................. 214 
Table C13: $\quad$ Survey II, Simplified TAM PCA: Component Correlation Matrix 214

\section{Appendix D Tables}

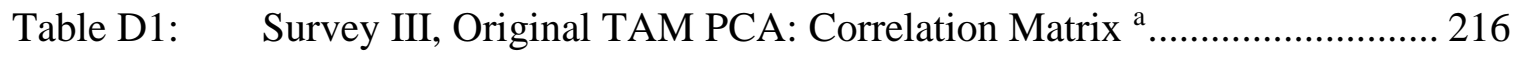

Table D2: $\quad$ Survey III, Original TAM PCA: Anti-image Correlation......................... 217

Table D3: $\quad$ Survey III, Original TAM PCA: KMO and Bartlett's Test...................... 217

Table D4: $\quad$ Survey III, Original TAM PCA: Communalities.................................... 218

Table D5: $\quad$ Survey III, Original TAM PCA: Total Variance Explained ..................... 219

Table D6: Survey III, Original TAM PCA: Structure Matrix.................................... 220

Table D7: $\quad$ Survey III, Original TAM PCA: Component Correlation Matrix ........... 220

Table D8: $\quad$ Survey III, Simplified TAM PCA: Correlation Matrix a ……………...... 221

Table D9: $\quad$ Survey III, Simplified TAM PCA: KMO and Bartlett's Test .................. 221

Table D10: $\quad$ Survey III, Simplified TAM PCA: Communalities ................................. 222

Table D11: $\quad$ Survey III, Simplified TAM PCA: Total Variance Explained ................ 222

Table D12: $\quad$ Survey III, Simplified TAM PCA: Structure Matrix ............................... 223

Table D13: $\quad$ Survey III, Simplified TAM PCA: Component Correlation Matrix ....... 223

\section{Appendix E Tables}

Table E1: $\quad$ Survey I, Original TAM PA: PEOU and PU Correlations ..................... 225

Table E2: $\quad$ Survey I, Original TAM PA: PEOU $\rightarrow$ PU Model Summary ………..... 225

Table E3: $\quad$ Survey I, Original TAM PA: PEOU $\rightarrow$ PU Coefficients ${ }^{a}$....................... 225

Table E4: Survey I, Original TAM PA: PU, PEOU, and ATT Correlations............ 226

Table E5: $\quad$ Survey I, Original TAM PA: PEOU and PU $\rightarrow$ ATT Model Summary 226

Table E6: $\quad$ Survey I, Original TAM PA: PU and ATT $\rightarrow$ BI Coefficients ${ }^{a}$............. 226

Table E7: $\quad$ Survey I, Original TAM PA: PU, ATT, and BI Correlations .................. 227 
Table E8: $\quad$ Survey I, Original TAM PA: PU and ATT $\rightarrow$ BI Model Summary ....... 227

Table E9: $\quad$ Survey I, Original TAM PA: PU and ATT $\rightarrow$ BI Coefficients ${ }^{a}$............ 227

Table E10: $\quad$ Survey I, Simplified TAM PA: PU, PEOU, and BI Correlations ........... 228

Table E11: $\quad$ Survey I, Simplified TAM PA: PEOU $\rightarrow$ PU Model Summary ............ 228

Table E12: $\quad$ urvey I, Simplified TAM PA: PEOU $\rightarrow$ PU Coefficients ${ }^{\text {a }}$................... 228

Table E13: $\quad$ Survey I, Simplified TAM PA: PU and PEOU $\rightarrow$ BI Model Summary. 229

Table E14: $\quad$ Survey I, Simplified TAM PA: PU and PEOU $\rightarrow$ BI Coefficients ${ }^{a} \ldots . . .229$

\section{Appendix F Tables}

Table F1: $\quad$ Survey II, Original TAM PA: PEOU and PU Correlations .................... 231

Table F2: $\quad$ Survey II, Original TAM PA: PEOU $\rightarrow$ PU Model Summary............... 231

Table F3: $\quad$ Survey II, Original TAM PA: PEOU $\rightarrow$ PU Coefficients ${ }^{\text {a }}$................... 231

Table F4: $\quad$ Survey II, Original TAM PA: PU, PEOU, and ATT Correlations ......... 231

Table F5: $\quad$ Survey II, Original TAM PA: PEOU and PU $\rightarrow$ ATT Model Summary 232

Table F6: $\quad$ Survey II, Original TAM PA: PEOU and PU $\rightarrow$ ATT Coefficients ${ }^{\text {a }} . . .232$

Table F7: $\quad$ Survey II, Original TAM PA: PU, ATT, and BI Correlations................ 232

Table F8: $\quad$ Survey II, Original TAM PA: PU and ATT $\rightarrow$ BI Model Summary ..... 233

Table F9: $\quad$ Survey II, Original TAM PA: PU and ATT $\rightarrow$ BI Coefficients ${ }^{\text {a }}$............ 233

Table F10: $\quad$ Survey II, Simplified TAM PA: PU, PEOU, and BI Correlations ......... 234

Table F11: $\quad$ Survey II, Simplified TAM PA: PEOU $\rightarrow$ PU Model Summary ........... 234

Table F12: $\quad$ Survey II, Simplified TAM PA: PEOU $\rightarrow$ PU Coefficients ${ }^{\text {a }}$................ 234

Table F13 Survey II, Simplified TAM PA: PU and PEOU $\rightarrow$ BI Model Summary

Table F14: $\quad$ Survey II, Simplified TAM PA: PU and PEOU $\rightarrow$ BI Coefficients ${ }^{\text {a }}$.... 235 


\section{Appendix G Tables}

Table G1: $\quad$ Survey III, Original TAM PA: PEOU and PU Correlations.................. 237

Table G2: $\quad$ Survey III, Original TAM PA: PEOU $\rightarrow$ PU Model Summary …......... 237

Table G3: $\quad$ Survey III, Original TAM PA: PEOU $\rightarrow$ PU Coefficients ${ }^{\text {a }}$.................. 237

Table G4: $\quad$ Survey III, Original TAM PA: PU, PEOU, and ATT Correlations ........ 238

Table G5: $\quad$ Survey III, Original TAM PA: PEOU and PU ATT Model Summary... 238

Table G6: $\quad$ Survey III, Original TAM PA: PEOU and PU $\rightarrow$ ATT Coefficients a ${ }^{2 . .2} 238$

Table G7: $\quad$ Survey III, Original TAM PA: PU, ATT, and BI Correlations .............. 239

Table G8: $\quad$ Survey III, Original TAM PA: PU and ATT $\rightarrow$ BI Model Summary .... 239

Table G9: $\quad$ Survey III, Original TAM PA: PU and ATT $\rightarrow$ BI Coefficients ${ }^{\text {a }}$......... 239

Table G10: $\quad$ Survey III, Simplified TAM PA: PU, PEOU, and BI Correlations ........ 240

Table G11: $\quad$ Survey III, Simplified TAM PA: PEOU $\rightarrow$ PU Model Summary .......... 240

Table G12: $\quad$ Survey III, Simplified TAM PA: PEOU $\rightarrow$ PU Coefficients ${ }^{\text {a }}$............... 240

Table G13: $\quad$ Survey III, Simplified TAM PA: PU and PEOU $\rightarrow$ BI Model Summary

Table G14: $\quad$ Survey III, Simplified TAM PA: PU and PEOU $\rightarrow$ BI Coefficients ${ }^{\text {a }} . . .241$ 


\section{LIST OF FIGURES}

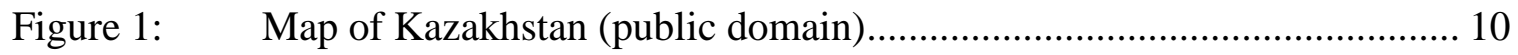

Figure 2: $\quad$ The Technology Acceptance Model (TAM) (Davis et al., 1989)............ 29

Figure 3: $\quad$ Theory of Reasoned Action (TRA) (Ajzen \& Fishbein, 1980) ................ 30

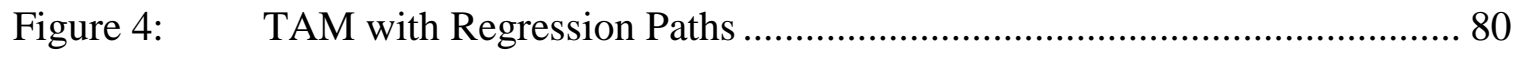

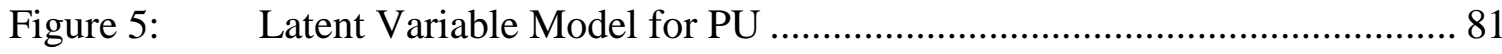

Figure 6: $\quad$ Survey I Results (Original TAM) …............................................ 105

Figure 7: $\quad$ Survey Results (Simplified TAM) ................................................ 106

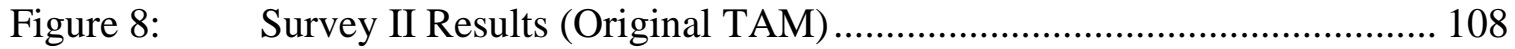

Figure 9: $\quad$ Survey II Results (Simplified TAM) ............................................ 109

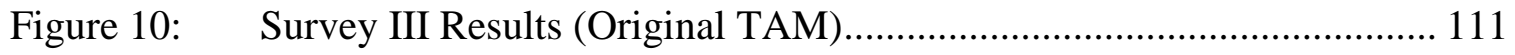

Figure 11: $\quad$ Survey III Results (Simplified TAM) ........................................... 112

Figure 12: $\quad$ Visits Over Time (Researcher's students) ........................................ 120

Figure 13: $\quad$ Visits Over Time (Guest access).................................................... 124 


\section{LIST OF ABBREVIATIONS}

LMS

ICT

IT

IS

EDM

LA

PCA

PC

PA

SEM

PD

TAM

PEOU

PU

ATT

BI

CVL

COP
Learning Management System

Information Communication Technology

Information Technology

Information Systems

Educational Data Mining

Learning Analytics

Principal Component Analysis

Principal Components

Path Analysis

Structural Equation Modeling

Power Distance

Technology Acceptance Model

Abbreviations for TAM Factors

Perceived Ease of Use

Perceived Usefulness

Attitude Toward Using

Behavioral Intention to Use

Abbreviations for Survey Codes

Course Video Lectures

Course Online Presentations 


$\begin{array}{ll}\text { CLA } & \text { Course Laboratory Assignment } \\ \text { CAF } & \text { Course Announcements Forums } \\ \text { COG } & \text { Course Online Grades } \\ \text { DEM } & \text { Demographics } \\ \text { PEX } & \text { Previous Elearning Experience } \\ \text { TEC } & \text { Technology Skill } \\ \text { TR } & \text { Technology Readiness } \\ \text { LR } & \text { Learner Readiness } \\ \text { CR } & \text { Content Readiness } \\ \text { PU-VR } & \text { Perceived Usefulness of Video Resources } \\ \text { PU-PR } & \text { Perceived Usefulness of Online Presentations } \\ \text { PU-LAB } & \text { Perceived Usefulness of ICT Laboratory Studies } \\ \text { PU-AN } & \text { Perceived Usefulness of Course Announcements } \\ \text { PU-OG } & \text { Perceived Usefulness of Online Grades }\end{array}$




\section{CHAPTER ONE: INTRODUCTION}

The technology acceptance model (TAM) is one method that educational researchers use to understand levels of perceptions in elearning systems (Liaw, Huang, \& Chen, 2007; Ngai, Poon, \& Chan, 2007; S. Y. Park, 2009; Shroff, Deneen, \& Ng, 2011). TAM is an information systems theory that demonstrates how the users' perceived ease of use and perceived usefulness of the system directly influence their attitude, which is an accurate predictor of usage (Davis, Bagozzi, \& Warshaw, 1989). Furthermore, perceptions of elearning are one measure of elearning readiness, which seeks to determine how prepared an organization is mentally or physically to engage in elearning (Borotis \& Poulymenakou, 2004). The primary factors of TAM that determine actual system use are perceived usefulness (PU), perceived ease of use (PEOU), attitude toward using (ATT), and behavioral intention to use (BI).

\section{Elearning}

Elearning is commonplace in many universities and workplaces, particularly in Western countries (Cheng, Wang, Mørch, Chen, \& Spector, 2014; Docebo, 2016). This research project defines elearning as the "confluence of educational psychology and instructional design, of educational technology and distance education, and of recent technological developments related to the Internet" (Friesen, 2009, p. 6). Examples of elearning methods include instructor-led, self-paced (Liaw et al., 2007), synchronous, asynchronous (Hrastinski, 2008), fully online (Anderson, 2004), and blended learning (Garrison \& Kanuka, 2004; Singh, 2003). Blended learning is on a spectrum between 
technology-enhanced learning and fully-online learning that is "the thoughtful integration of classroom face-to-face learning experiences with online learning experiences" (Garrison \& Kanuka, 2004, p. 96). The flipped classroom is one instructional strategy that uses blended learning to change the classroom activities from disseminating information to knowledge application (O’Flaherty \& Phillips, 2015).

Countries in the developing world struggle to adopt elearning methodologies regardless of the benefits. Researchers show that elearning improves access to learning materials, and provides flexibility for students to choose when and where to study (Alexander, 2001; Bhuasiri, Xaymoungkhoun, Zo, Rho, \& Ciganek, 2012; Mouyabi, 2011). However, cultural issues (Gulati, 2008; Mumtaz, 2000) and traditional educational practices (Ahmad Al-Adwan \& Smedley, 2012; de la Sablonnière, Taylor, \& Sadykova, 2009) are hindrances to educational progress in developing countries. These are significant issues because a crucial prerequisite for the successful implementation of elearning projects is favorable attitudes toward elearning (Bhuasiri et al., 2012).

\section{Kazakhstan}

Kazakhstan is a developing nation that has interests in implementing elearning initiatives, but traditional educational methods create challenges in using elearning methodologies. The report on education reforms in Kazakhstan found that rote-learning is still common in Kazakh secondary schools, which inhibits the necessary development of critical thinking skills (Nazarbayev University Graduate School of Education [NUGSE], 2014). The report also noted the common practice of using educational technology as an extension of textbooks, instead of using a variety of resources that are "interactive and challenging” (2014, p. 30). 
Culture impacts the development of educational systems (Gulati, 2008; Mumtaz, 2000) and research demonstrates its importance in elearning readiness (Borotis \& Poulymenakou, 2004; Lopes, 2007). Researchers have attempted to create a cultural profile of Kazakhstan using Hofstede's (1980) dimensions. The studies depict the country as being high in collectivism, masculinity, power distance (PD), and uncertainty avoidance (Chung \& Holdsworth, 2012) with a preference toward long-term planning (Ardichvili \& Kuchinke, 2002; Nezhina \& Ibrayeva, 2013).

The limited research using the socio-cultural dimensions in Kazakhstan shows discrepancies and reveals the complexity of the Kazakhstani culture (Ismail \& Ford, 2010; Mukazhanova, 2012). An example of a discrepancy is that three separate studies rated Kazakhstan's PD as low (Ardichvili \& Kuchinke, 2002), medium (Karibayeva \& Kunanbayeva, 2017), and high (Ismail \& Ford, 2010). One complexity that could explain the differing results is the coexistence of dissimilar cultures, each having their own language, cultural values, and history. The majority ethnic population in Kazakhstan is Turkic speaking and Muslim, but a represented minority speaks a Slavic language and are historically Orthodox Christians ("Ethnic demography of Kazakhstan," 2017; Nezhina \& Ibrayeva, 2013). Even though the Slavic-speaking minorities only represent approximately $25 \%$ of the population, the groups are concentrated closer to the Russian border (“Ethnic demography of Kazakhstan," 2017). The results from prior studies could be different depending on the population that the researcher used to obtain samples. Therefore, there is a need to further explore elearning readiness in Kazakhstan using existing cultural data. 


\section{Using TAM to Determine Elearning Readiness}

Research demonstrates that TAM is an accurate predictor of usage in the North American context. Based on the success of TAM in North America, educational researchers use TAM in different cultures, such as Spain (Padilla-MeléNdez, Del AguilaObra, \& Garrido-Moreno, 2013), Greece, (Tselios, Daskalakis, \& Papadopoulou, 2011), Singapore (T. Teo, 2011), Hong Kong (Ngai et al., 2007), South Korea (S. Y. Park, 2009), and Saudi Arabia (Al-Gahtani, 2016). However, researchers argue that TAM does not produce accurate results when applied across cultures (McCoy, Galletta, \& King, 2007; Straub, Keil, \& Brenner, 1997). Huang, Lu, \& Wong's (2003) study on the effect of power distance "empirically establishes a theoretical link between cultural factors and technology adoption"' (2003, p. 98). McCoy et al.’s (2007) study using participants from 24 different countries revealed that TAM was not a consistent predictor for societies with Hofstede's (1980) cultural dimensions of low uncertainty avoidance, high PD, high masculinity, and high collectivism. The limited available research, described in the next chapter, shows that Kazakhstan could have the cultural dimensions of high PD, masculinity, and collectivism (Nezhina \& Ibrayeva, 2013).

Research using TAM to predict system usage shows a range of cultural considerations that include testing the model in a specific context, discussing cultural aspects when using the model, mentioning it in passing, or not mentioning it at all. TAM investigators have considered cultural implications and tested TAM in developing countries, such as Saudi Arabia (Al-Gahtani, 2016; Baker, Al-Gahtani, \& Hubona, 2010), Lebanon (Tarhini, Hone, \& Liu, 2013, 2014; Tarhini, Hone, Liu, \& Tarhini, 2017), and Jordan (Amer Al-Adwan, Al-Adwan, \& Smedley, 2013). Other TAM analyses briefly 
acknowledge or mention the cultural limitations of the model (Hsu \& Lu, 2004; Roca, Chiu, \& Martínez, 2006; Wu, Tennyson, \& Hsia, 2010; Yang \& Yoo, 2004). Additionally, it is not uncommon for TAM studies used to predict system usage in educational settings to omit cultural considerations entirely (Lee, Cheung, \& Chen, 2005; Liaw, Chen, \& Huang, 2008; Liaw et al., 2007; Ngai et al., 2007; N. Park, Roman, Lee, \& Chung, 2009; S. Y. Park, 2009; Shroff et al., 2011; Šumak, Heričko, Pušnik, \& Polančič, 2011). Because of the cultural influence on TAM and the lack of research in developing countries, additional research is still needed in different cultural contexts.

\section{Purpose of the Study}

Student readiness is a crucial success factor in the adoption of elearning. Researchers have conducted intensive studies in western countries using TAM, but there is a lack of research that discusses elearning initiatives and acceptance in the developing world, such as the broader Central Asian region, and specifically Kazakhstan.

Furthermore, TAM is not a consistent predictor across cultures, specifically in developing countries. Due to the success of TAM in the developed world, researchers assume the model works across cultures, which could lead to inaccurate results. Therefore, this study aimed to investigate students' perceptions and readiness of using a learning management system (LMS) in Central Asian countries, using Kazakhstan as the example. Specifically, this study examined if the TAM factors of PU, PEOU, ATT, and BI in an elearning setting in Kazakhstan are the same factors as those identified in the developed countries to determine if TAM is a consistent predictor across cultures. 


\section{Expected TAM Results}

This study lists assumptions of how TAM behaves in developed countries and how TAM should perform in Kazakhstan. In developed countries, the expected TAM results are that:

1. All TAM paths are significant on successful TAM implementations.

2. PU is the stronger influencer of $\mathrm{BI}$ (King \& He, 2006).

3. PEOU is the weakest predictor of actual usage (Turner, Kitchenham, Brereton, Charters, \& Budgen, 2010).

4. BI is an accurate predictor of actual usage (Turner et al., 2010).

As shown in Table 1, the path of PEOU $\rightarrow$ BI should not be significant given Kazakhstan's cultural dimension. A prediction cannot be made without additional data on the path of PU $\rightarrow$ BI because PU does not significantly affect BI in high PD cultures (Huang et al., 2003), but it does in collectivistic and masculine cultures (McCoy et al., 2007).

Table 1: Expected Significant Paths for Kazakhstan (McCoy et al., 2007)

\begin{tabular}{cccc}
\hline $\mathrm{PU} \rightarrow \mathrm{BI}$ & $\mathrm{PEOU} \rightarrow \mathrm{BI}$ & $\mathrm{PEOU} \rightarrow \mathrm{PU}$ & Hofstede's (1980) Dimension \\
\hline $\mathrm{No}^{\mathrm{a}}$ & $\mathrm{No}^{\mathrm{a}}$ & Yes & High power distance \\
Yes & $\mathrm{No}^{\mathrm{b}}$ & Yes & High collectivism \\
Yes & $\mathrm{No}^{\mathrm{c}}$ & Yes & High masculinity \\
\hline
\end{tabular}

a. Supported by Huang, Lu, \& Wong (2003).

b. Supported by Abbasi, Tarhini, Elyas, and Shah (2015).

c. Supported by Srite \& Karahanna (2006).

\section{Research Questions}

1. Does PU have a stronger influence than PEOU on ATT in Kazakhstan?

2. How do the significant factors of PU, PEOU, ATT, and BI in Kazakhstan compare to the significant factors in developed countries? 
The comparative analysis used for research question number two uses a qualitative approach to subjectively compare the TAM results from Kazakhstan to TAM studies that are listed in Tables 6-8 using two criteria. The first criterion is that the path is significant. If it is not significant, then an evaluation is made based on if the path is significant most of the time in the baseline studies. Some paths, such as PEOU $\rightarrow$ PU, are usually significant whereas path PU $\rightarrow$ BI is significant less often (King \& He, 2006).

\section{Method Overview}

The quantitative research method for this project used survey research for data collection, principal component analysis for component extraction, and path analysis for the analysis of TAM.

\section{Table 2: $\quad$ Project Overview}

\begin{tabular}{ll}
\hline \multicolumn{1}{c}{ Item } & \multicolumn{1}{c}{ Description } \\
\hline Problem & $\begin{array}{l}\text { TAM is not a consistent predictor across cultures } \\
\text { (McCoy et al., 2007). }\end{array}$ \\
\hline Central Question & Is TAM an accurate predictor in Kazakhstan? \\
\hline Task & $\begin{array}{l}\text { Used TAM to predict student acceptance of elearning at a } \\
\text { university in Kazakhstan. }\end{array}$ \\
\hline Elearning Methodology & $\begin{array}{l}\text { Facilitated blended learning in a face-to-face class using an } \\
\text { LMS. }\end{array}$ \\
\hline Research Method & $\begin{array}{l}\text { Survey research (Creswell, 2014; Ruel, Wagner III, \& } \\
\text { Gillespie, 2015) }\end{array}$ \\
\hline Statistical Analysis & $\begin{array}{l}\text { Principal Component Analysis (O'Rourke \& Hatcher, 2013) } \\
\text { Path Analysis (Duncan, 1966; Garson, 2008) }\end{array}$ \\
\hline Comparative Analysis & $\begin{array}{l}\text { Qualitatively compared TAM results in Kazakhstan to results } \\
\text { of TAM studies in developed countries. }\end{array}$ \\
\hline Scientific Merit & $\begin{array}{l}\text { Determined if TAM is an acceptable method to predict usage } \\
\text { of elearning systems in Kazakhstan. }\end{array}$ \\
\hline Broader Impacts & $\begin{array}{l}\text { (a) Researchers in other developing countries can have data } \\
\text { on how the cultural dimensions of high PD, high } \\
\text { masculinity, and high collectivism affect TAM. }\end{array}$ \\
\hline
\end{tabular}


(b) Call into question TAM studies in other developing countries if TAM is not a consistent predictor in cultures with the dimensions of high PD, high masculinity, and high collectivism.

Local Impacts

A university in East Kazakhstan has first-hand data and experience of what their students think about elearning.

\section{Glossary of Terms}

This section defines frequently used terms that are unclear, misunderstood, or have varying definitions based on context and prior knowledge.

Elearning

The "confluence of educational psychology and instructional design, of educational technology and distance education, and of recent technological developments related to the Internet" (Friesen, 2009, p. 6).

High collectivism or collectivist

A societal characteristic where people are integrated into groups where they are protected in exchange for loyalty (Hofstede, 2011).

High Masculinity

A society where there are gaps between men's and women's values, admiration for the strong, and women seldom in elected positions (Hofstede, 2011).

High power distance (PD) A culture where the less powerful members of organizations and institutions accept and expect that power is distributed unequally (Hofstede, 2011). 
Kazakh

Kazakhstani

Learning Management System (LMS)

Original TAM

Simplified TAM
A Turkic ethnic people group native to Kazakhstan or something relating to the Kazakh people.

A citizen of Kazakhstan.

A generalized term for an all-in-one online system

that has administrative and learning features to benefit

both the educator and the student.

Davis et al.'s (1989) model that includes the four

factors of PU, PEOU, ATT, and BI.

Omits the ATT construct and uses the three factors of

PU, PEOU, and BI (Venkatesh \& Bala, 2008;

Venkatesh \& Davis, 1996). 


\section{CHAPTER TWO: THE KAZAKHSTAN CONTEXT}

\section{Overview}

Kazakhstan is a Central Asian country that shares borders with Russia, China, Kyrgyzstan, Uzbekistan, and Turkmenistan. It became independent from the USSR in 1991. Kazakhstan is the ninth-largest country ranked according to landmass but ranks $236^{\text {th }}$ by population density with a population of 18.3 million (“Kazakhstan," 2018). Kazakhstan is a multi-ethnic country with 131 ethnicities, with the largest ethnicities being Kazakhs, Russians, Uzbeks, Ukrainians, Germans, Tatars, and Uyghurs ("Kazakhstan,” 2018). Kazakhs represent up 63\% of the population ("Ethnic demography of Kazakhstan,”2017).

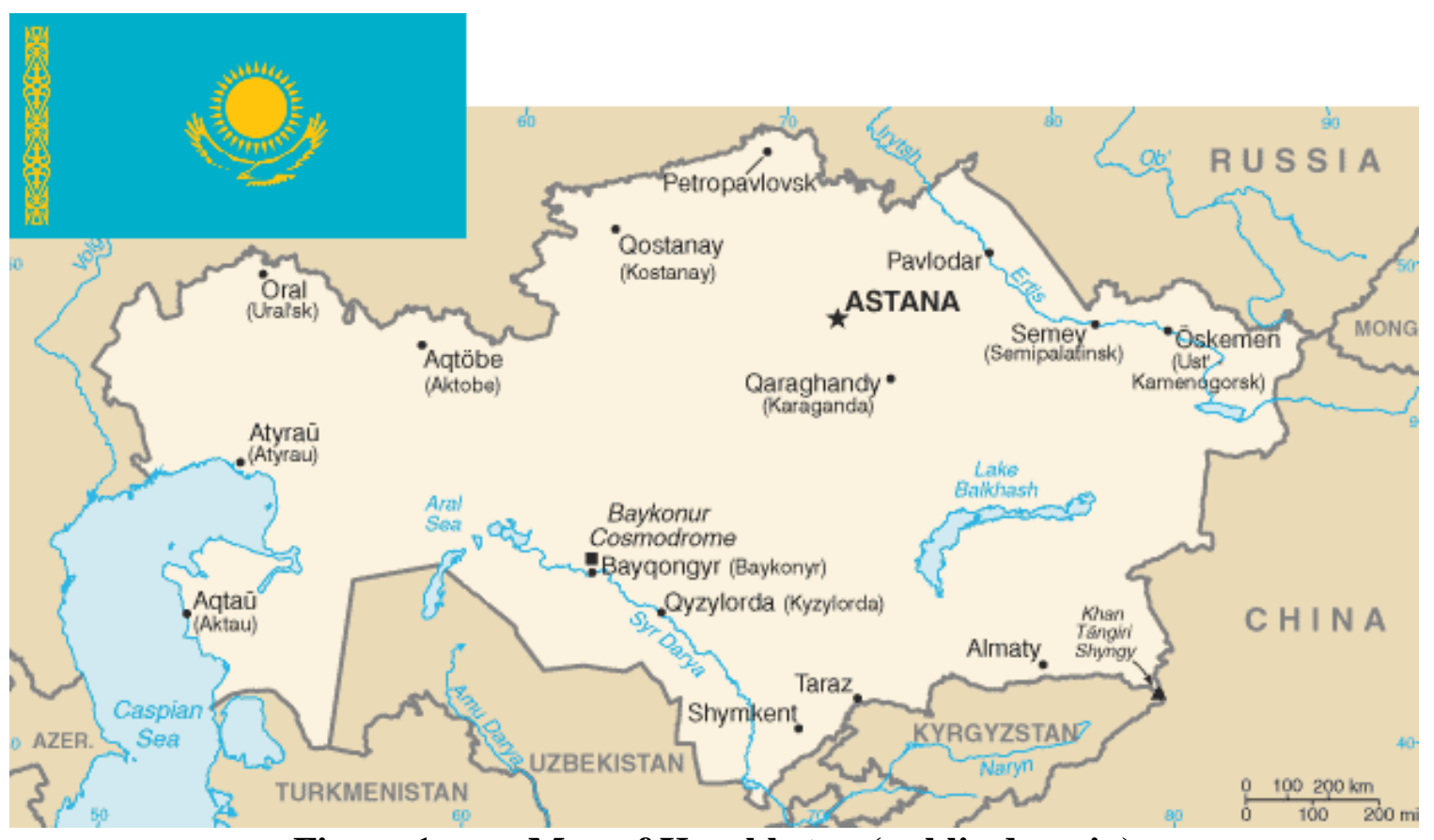

Figure 1: $\quad$ Map of Kazakhstan (public domain) 
This chapter provides detailed background information about Kazakhstan because the context is central to the study. The major sections are developing nations, elearning in developing countries, elearning in Kazakhstan, and Hofstede's cultural profile of Kazakhstan. After laying the groundwork for the classification of developing nations, the next segment explores elearning projects and success criteria in the less economically developed countries. Then, this chapter examines the elearning environment in Kazakhstan to review their ambitions and issues that they face. The final part delves into a cultural profile of Kazakhstan that details the problems of trying to classify the country using Hofstede's socio-cultural dimensions.

\section{Developing Nations}

There is no precise definition of a developing country. One binary classification of countries is dividing them between developing or developed nations or less and more economically developed countries. The World Economic Situation and Prospects (WESP) classifies all countries in the three categories of developed economies, economies in transition, and developing economies for analytical purposes (UN/DESA, 2014). Another classification is the human development index (HDI) that considers factors other than economic development. HDI calculates a country's ranking based on its life expectancy, education, and per capita income indicators (United Nations Development Programme, 2016). The HDI categories are very high, high, medium, and low human development. This research project classifies only those countries ranked in the very high human development category as developed countries, and all other countries are developing countries. 
Accordingly, this project considers Kazakhstan a developing nation based on the WESP and HDI rankings. The 2014 WESP report lists Kazakhstan both as an economy in transition and as a developing country (UN/DESA, 2014). The 2016 United Nations Development Programme report lists Kazakhstan as a developing country in the multidimensional poverty index rating (United Nations Development Programme, 2016).

\section{Elearning in Developing Countries}

Countries in the developing world have difficulties adopting elearning methodologies. Research reveals educational problems in developing countries, but studies demonstrate how distance learning can address these challenges. One category of obstacles results from infrastructure and economic issues restricting educational progress and reform (Gulati, 2008). In the Pakistani context, these hindrances include a shortage of qualified and skilled teachers, low level of learning achievements, high drop-out rates at all levels of education, lack of opportunities for remote areas, and lack of study materials and resources (Nawaz, 2013). Researchers argue that distance learning is economically advantageous to training local classroom teachers (Ngampornchai \& Adams, 2016; Perraton, 2005), reduces the cost of traditional education, provides learning opportunities to rural learners (Aggarwal, 2009; Nawaz, 2013; Ngampornchai \& Adams, 2016), improves access to learning materials, and allows for students to choose their time and place of study (Alexander, 2001; Bhuasiri et al., 2012; Mouyabi, 2011).

Another category that hinders educational progress stems from cultural issues (Gulati, 2008; Mumtaz, 2000) and traditional educational practices (Ahmad Al-Adwan \& Smedley, 2012; de la Sablonnière et al., 2009). These are substantial issues because an 
essential prerequisite for the successful implementation of elearning projects is positive attitudes toward elearning (Bhuasiri et al., 2012).

One example of adhering to traditional educational methods is found in Kyrgyzstan, which still embraces the values of the Soviet education system where the teacher is the expert and dispenser of knowledge (de la Sablonnière et al., 2009). This educational philosophy impedes the acceptance of a student-centered method of teaching and "a change in approach signifies that both teachers and students change their attitudes and behaviors to education" (2009, p. 3).

A study from Jordan showed that students favor traditional classes and lectures to help them understand the content and that "students depended significantly on their teachers" for their educational progress (Ahmad Al-Adwan \& Smedley, 2012, p. 132). The study also revealed that students do not prefer to work independently but indicate "that face-to-face contact with tutors was a vital part of their learning" (2012, p. 131). The study cites several contributing factors that include students lacking information communication technology (ICT) skills, computers at home, and self-motivation for taking control of their learning (Ahmad Al-Adwan \& Smedley, 2012).

Developing countries recognize the benefits of elearning, and they are taking steps to overcome the issues inhibiting educational access. For example, Asian mobile network operators and device makers are engaging in elearning to address infrastructure issues (Ambient Insight, 2014). Ten developing countries had the highest growth rate of self-paced learning in Asia, with Myanmar having the most growth (Ambient Insight, 2014). A province in India is supplying 1.5 million laptops to primary and secondary 
students, and a region in Pakistan is providing 3G-enabled laptops to students in higher education (Ambient Insight, 2014).

Both educational institutions and national governments across the globe are researching or implementing elearning to support education. In the Nigerian state of Ebonyi, Agbo (2015) investigated using ICTs to teach computers to students at school and home. In China, Aixia and Wang (2011) researched utilizing an elearning platform as an alternative to traditional face-to-face education where teachers and students can share knowledge. The Peruvian government created an elearning portal called Peru EDUCA, which is one of the several initiatives aimed at modernizing their education system using ICT (Paola Torres Maldonado, Feroz Khan, Moon, \& Jeung Rho, 2011). The Ministry of Higher Education in Saudi Arabia started the National Centre for E-learning and Distance Learning to provide educational tools, services, and solutions to local universities (Alharbi \& Drew, 2014). In Kazakhstan, the Ministry of Education and Science started a large-scale project called e-Learning to provide a centralized learning and administrative platform for secondary schools ("e-Learning," 2013).

There are benefits and challenges to developing countries that implement elearning (Gunga \& Ricketts, 2007; Mouyabi, 2011; Sife \& Lwoga, 2007). Two specific ways that educational institutions benefit from elearning are improved access to learning materials and flexibility for students to focus on areas of needs or interests (Alexander, 2001; Bhuasiri et al., 2012; Mouyabi, 2011). Increased access allows students to view the digital materials at the times of their choosing. The online materials allow the students to review information and concentrate on topics they need to learn. 
Challenges of providing students with online access to digital materials require additional work, resources, and expertise on the institution's part. The ICT department at the institute should implement a learning environment or platform for the resources and maintain the system. The faculty's role is to digitize their materials and place the resources online in a way that promotes learning. Kamba (2009) explains that Nigerian universities lack experienced ICT professionals who are capable of supporting elearning implementation, and the staff and students lack the know-how on using an elearning system.

\section{Elearning in Kazakhstan}

Kazakhstan has many opportunities to use elearning in ways that support the country's educational initiatives and overcome barriers. The needs for distance learning in Kazakhstan include a dispersed population, understaffed and under-resourced schools, flexible learning opportunities for adult learners, and isolated individuals with physical disabilities (NUGSE, 2014). One specific way that elearning can help is to provide digital alternatives to compensate for the lack of physical libraries in high schools (Dalayeva, 2013). The NUGSE report recommends the use of radio and television to reach rural areas affected by the digital divide, which states that internet access "is available in 99\% of schools, though broadband is available in only 43\% of schools" (NUGSE, 2014, p. 45). As a developing nation, Kazakhstan faces problems that are significant barriers to elearning. The main obstacles to distance learning are "the low level of digital literacy of teachers, inadequate technical equipment in rural areas and non-system development of distance learning in universities" (Sapargaliyev, 2012, p. 3). 
The government in Kazakhstan directs and controls elearning projects, some of which are part of more extensive strategic plans (Abdiraiymova, Burkhanova, \& Kenzhakimova, 2012; NUGSE, 2014; Sapargaliyev, 2011). Additionally, the state has established standards and regulations for using distance learning and elearning in Kazakhstani educational institutions (Dalayeva, 2013; Sapargaliyev, 2011). The education reforms in Kazakhstan for 2015-2020 include exploring elearning options in over 800 schools and developing the Centers of Excellence programme to "promote and equip teachers with more versatile pedagogies" (NUGSE, 2014, p. 30). A previous example of an elearning initiative started under the Ministry of Education and Science was to create a mobile electronic catalog for the delivery of audio and video lectures to mobile devices (Sapargaliyev, 2011). Another example is the conference on ICTs in education carried out by the Ministry of Education (Sapargaliyev, 2011).

Research identifies areas of need in Kazakhstan's distance and elearning initiatives that address their educational needs. NUGSE's (2014) evaluation of Kazakhstan's 2015-2020 education reforms offered a critique of their inadequate distance learning planning. The report states that the authors "were quite surprised not to find any of the sophisticated approaches to distance education that have been developed at all levels in countries like Australia and Canada, with similarly widely scattered populations" (NUGSE, 2014, p. 30). One problem is that some initiatives do not take a comprehensive approach when using elearning. For example, NUGSE (2014) explains that while the Centers of Excellence programme trained 10,000 teachers in new pedagogical methods, the curriculum still reflects traditional approaches to education. 
Furthermore, the report acknowledges training problems with the pilot project to introduce elearning in 800 schools:

Our limited evidence suggests, however, that few teachers have grasped the possibilities that web-based learning might offer. In our classroom observations, teachers still tend to use e-learning resources as an extension of the textbook rather than as a much more diverse, interactive and challenging resource for learning. (NUGSE, 2014, p. 30)

\section{Hofstede's Profile of Kazakhstan}

Studies show that culture affects educational progress (Gulati, 2008; Mumtaz, 2000) and that it is a criterion for evaluating elearning readiness (Lopes, 2007). For these reasons, examining Kazakhstan's culture can help identify issues with implementing elearning methodologies in the country.

Problems with Existing Literature

There is limited research on Hofstede's socio-cultural value dimensions specifically in Kazakhstan (Ardichvili \& Kuchinke, 2002; Ismail \& Ford, 2010; Karibayeva \& Kunanbayeva, 2017; Mukazhanova, 2012; Nezhina \& Ibrayeva, 2013). Hofstede's $(1984,1997)$ five cultural dimensions are power distance (PD), individualism/collectivism, uncertainty avoidance, masculinity/femininity, and longterm/short-term planning. Ardichvili and Kuchinke (2002) stated that data on these cultural dimensions "could not be found" (2002, p. 101) for Kazakhstan. Karibayeva and Kunanbayeva (2017) note that the PD for Kazakhstan was not available in literature preceding their study.

Additionally, prior researchers base their claims on assumptions or generalizations. Chung and Holdsworth (2012) make assertions that Kazakhstan has the dimensions of "high collectivism, high uncertainty avoidance, high PD and high 
masculinity" (2012, p. 225). However, they arrive at that conclusion based on Kazakhstan being a collectivist culture from prior research and a conference paper presented in Anchorage, Alaska in 2000. Vasile and Nicolescu's (2016) study does not present a literature review or a basis for their research, but they only report the findings of their survey. Ismail and Ford's (2010) examination of the literature on leadership and cultural dimensions in Central Asia and the Caucasus results in contradictory findings and generalizations of cultural similarities with Russia and Eastern European countries based on the shared Soviet heritage.

Mukazhanova's (2012) master's thesis notes the lack of empirical data used to determine cultural dimensions in Kazakhstan. Instead, she explains that the studies generalize the culture based on geopolitical history. The 2004 GLOBE study (House, Hanges, Javidan, Dorfman, \& Gupta, 2004) puts Kazakhstan with the Eastern European cluster, which includes the countries of Albania, Georgia, Greece, Hungary, Poland, Russia, and Slovenia. However, Ismail and Ford (2010) and Mukazhanova (2012) argue that Central Asian culture is distinctly different from that of Eastern Europe.

Mukazhanova (2012) describes how Kazakh nomadic and tribal history gives the country distinctions not found in the Eastern European countries. Ismail and Ford (2010) note a similar sentiment with the GLOBE study in relation to their leadership investigation and state that a sample should be "from a broad cross-section of countries from Central Eurasia in a manner that does not confound the outcomes by including these countries in geographically dissimilar clusters such as Eastern Europe” (2010, p. 330). 


\section{$\underline{\text { Results from Empirical Studies }}$}

Empirical studies attempt to classify Kazakhstan using Hofstede's socio-cultural dimensions. The diverse Kazakhstani population adds complexity to providing an accurate cultural description since the country is multi-cultural. One example is that the two primary ethnic populations are dissimilar since one is Turkic and Muslim and the other is Slavic and Orthodox ("Ethnic demography of Kazakhstan," 2017; Nezhina \& Ibrayeva, 2013), each having their unique history, language, and culture.

Ardichvili and Kuchinke (2002) surveyed 4,200 employees and managers in ten organizations across six countries and provided human resource development comparisons using five of Hofstede's socio-cultural dimensions (Hofstede, 1984, 1997), which are PD, individualism, masculinity, uncertainty avoidance, and long-term orientation. Ardichvili and Kuchinke's (2002) results showed that, as compared to the US and Germany, the former Soviet Republics of Russia, Georgia, Kazakhstan, and Kyrgyzstan had significantly lower levels of PD, higher levels of masculinity, and preferred long-term planning.

Karibayeva and Kunanbayeva (2017) sought to measure the Hofstede's PD and verbal index for Kazakh culture. Their method included analyzing the transcripts of various world leaders' speeches to look for low and high power level words or phrases. Word examples include the addresses where the leaders used "I" or "must" as compared to "we" or "together." Karibayeva and Kunanbayeva also surveyed 133 participants from a wide range of industries. They report that the Hofstede PD index in Kazakhstan is 58, which is medium on the 1-120 PD index scale. 
Nezhina and Ibrayeva's (2013) empirical study evaluated Hofstede's culture dimensions of PD, individualism, and uncertainty avoidance in Kazakhstan as it relates to cultural acceptance of nongovernmental organizations. They collected quantitative data from a survey and qualitative data by interviewing 30 individuals. The results of Hofstede's cultural dimensions describe Kazakhstan as having high PD (85\%), low individualism (15\%), and high uncertainty avoidance (80\%).

The survey of the literature indicates that Kazakhstan could have high collectivism (Chung \& Holdsworth, 2012; Ismail \& Ford, 2010; Nezhina \& Ibrayeva, 2013), high uncertainty avoidance (Chung \& Holdsworth, 2012; Ismail \& Ford, 2010), high masculinity (Ardichvili \& Kuchinke, 2002; Chung \& Holdsworth, 2012), and preferred long-term planning (Ardichvili \& Kuchinke, 2002; Nezhina \& Ibrayeva, 2013). There is not a consensus on the PD, which ranges from high (Chung \& Holdsworth, 2012; Ismail \& Ford, 2010), to medium (Karibayeva \& Kunanbayeva, 2017), to low (Ardichvili \& Kuchinke, 2002). 


\section{CHAPTER THREE: LITERATURE REVIEW}

\section{Overview}

This chapter provides background information and rationale support for the study. The major sections are elearning, the technology acceptance model (TAM), learning management systems (LMS), and data analytics in LMSs. The elearning segment introduces elearning, explores elearning readiness, and describes elearning success criteria. The technology acceptance model section gives an in-depth look at the model and using TAM in cultural, obligatory, and elearning contexts. The segment on learning management systems describes LMSs as educational tools and presents the justification for choosing Moodle, which is an open source LMS. The final section discusses data analytics in LMSs.

\section{Elearning}

\section{Defining Elearning}

The terms distance learning, elearning, and online learning evolve with the development of technology. Furthermore, researchers agree conceptually on the terms, but not on the details. As technology advances, some researchers broaden the definition to include additional technologies (Means, Toyama, Murphy, Bakia, \& Jones, 2009), while others narrow the terms (Friesen, 2009). Urdan and Weggen (2000) created a distance education framework to categorize subsets of distance learning. The four layers that clarify the specificity of distance education, from general to specific, are distance 
learning, elearning, online learning, and computer-based learning (Urdan \& Weggen, 2000).

Distance education is an educational setting where distance separates the instructors and students (Urdan \& Weggen, 2000). Urdan and Weggen (2000) defined distance learning as the delivery of education or training courses "to remote locations via synchronous or asynchronous means of instruction, including written correspondence, text, graphics, audio and videotape, CD-ROM, online learning, audio and videoconferencing, interactive TV, and facsimile" (2000, p. 88). Elearning is a subset of distance learning that is a "confluence of educational psychology and instructional design, of educational technology and distance education, and of recent technological developments related to the Internet and the Web" (Friesen, 2009, p. 6). An important aspect of Friesen's (2009) definition is the inclusion of educational methodologies, such as instructional design methods. With a focus on learning approaches, elearning shows intentionality instead of the dissemination of information. Garrison and Kanuka (2004) include the term "thoughtful integration" $(2004$, p. 96) to describe the incorporation of online learning experiences in blended learning. Online learning is the next layer of the distance learning model (Urdan \& Weggen, 2000). It describes learning that uses the specific mediums of network technologies, which are the internet, intranet, and extranet (Urdan \& Weggen, 2000). Means, Toyama, Murphy, Bakia, and Jones (2009) define online learning "as learning that takes place partially or entirely over the Internet" (2009, p. 9). They clarify that online learning "excludes purely print-based correspondence education, broadcast television or radio, videoconferencing, videocassettes, and standalone educational software programs that do not have a significant Internet-based 
instructional component" (Means et al., 2009, p. 9). Downes (2005) argues that elearning evolves with technology, which explains the variations of the details in the two layers. Literature indicates that many researchers and practitioners use online learning and elearning synonymously (Ahmad Al-Adwan \& Smedley, 2012; Liaw et al., 2007; Parkes, Stein, \& Reading, 2015; Sife \& Lwoga, 2007).

This research project only refers to the top two tiers of Urdan and Weggen's (2000) distance learning model with a focus on elearning, which are distance learning and elearning. This project uses Friesen's (2009) definition of elearning but excludes the limitation of using the web since communication technologies, such as WhatsApp, use the internet and not the web. Therefore, elearning is the "confluence of educational psychology and instructional design, of educational technology and distance education, and of recent technological developments related to the Internet" (Friesen, 2009, p. 6). $\underline{\text { Elearning Readiness }}$

Successfully implementing elearning methodologies is contingent on many factors that educators should evaluate before using elearning. These aspects include assessing the organization's readiness to adopt the principles and examining criteria to sustain the initiatives.

Elearning readiness is "the mental or physical preparedness of an organization for some e-Learning experience or action” (Borotis \& Poulymenakou, 2004, p. 1622). Elearning readiness or preparedness is not a binary value, but a measurement on a scale (Bessadok \& Abdulsalam, 2016; Borotis \& Poulymenakou, 2004). Bessadok and Abdulsalam's (2016) readiness study in Saudi Arabia revealed a scale of readiness for students who struggled with elearning. This progression of students contains those who 
have no intention of using elearning without explicit requirements, those who need convincing that elearning is beneficial, those who are convinced of elearning but have computer anxiety, and those who faced substantial obstacles and need help overcoming them.

In addition to being prepared mentally and physically, students must possess study skills such as time management, critical thinking skills, technical competencies, and collaborative learning skills. Parkes et al. (2015) evaluated student preparedness on a generation of students in Australia who are considered technically competent. The research team argues that being technology-savvy does not mean that students are suited for studying in online learning environments (Parkes et al., 2015). Their study revealed four significant findings about the preparedness of these students. First, students were poorly prepared in balancing their academic responsibilities, work, and personal lives in an elearning environment (Parkes et al., 2015). Next, the students had low levels of preparedness related to critical thinking skills and general academic skills (Parkes et al., 2015). Third, Parkes et al.'s (2015) study showed that students were adequately prepared in technical competencies relating to the internet. The study results indicate that typical web-browsing behavior is enough for students to transfer the skill to navigating course content. Furthermore, Parkes et al. (2015) suggest that students who lack experience using computer technology might struggle to use an LMS. Finally, students were moderately prepared for engaging in collaborative learning (Parkes et al., 2015).

Researchers have developed a variety of elearning readiness models for organizations to gauge the readiness level of various stakeholders or components (Aydin \& Tasci, 2005; Borotis \& Poulymenakou, 2004; Chapnick, 2000; Lopes, 2007; Psycharis, 
2005). Chapnick's (2000) readiness model looks at eight different stakeholders at a horizontal level within an organization with the aim of simplifying the process. Aydin and Tasci (2005) developed a table-based model to evaluate an organization's ability and willingness to adopt elearning (Mosa, Naz'ri bin Mahrin, \& Ibrrahim, 2016). The table has four rows of elements that are technology, innovation, people, and self-development to evaluate against three criteria-based columns of resources, skills, and attitudes (Aydin \& Tasci, 2005). Lopes (2007) modified Borotis and Poulymenakou's (2004) generic model to focus on higher educational institutes. The factors of Lopes' (2007) model are business, technology, content, culture, human resources, and finances. According to Lopes' (2007) model, the institute can only accept elearning "if it is aligned with business aims and objectives and leads to the effective accomplishment of the business aims" (Mosa et al., 2016, p. 119). Psycharis (2005) uses a tree-like structure to narrow the focus and systematize all the components in resources, education, and environment. These factors also have independencies, such as the environment influencing resources and specifying education (Psycharis, 2005).

A comparison of these models shows their differentiating approaches and weaknesses. For example, Hashim and Tasir's (2014) analysis of twelve elearning readiness assessment studies and instruments showed gaps in assessing readiness. They argue that most of the elearning readiness instruments that they analyzed lack the criteria for useful evaluation in emerging countries (Hashim \& Tasir, 2014). Mosa et al.'s (2016) evaluation of ten different elearning readiness models showed that technology was the common factor in all of them, with eight models listing learners as a factor. Content and resources were the next most frequent factors, showing up in six studies. Acceptance of 
elearning was part of only two models, which indicates that the researchers did not deem it as an essential criterion in determining readiness. The comparison of the factors of the technology aspect of elearning readiness showed that internet access, hardware, and availability of computers were the top items (Mosa et al., 2016).

The readiness model (Chapnick, 2000) lists eight factors used to measure an organization's elearning readiness, which are psychological, sociological, environmental, human resource, financial, technological skill, equipment, and content readiness. These criteria intend to answer the questions of (a) can we do this, (b) if we do this, how are we going to do it, and (c) what are the outcomes and how do we measure them (Chapnick, 2000)? Furthermore, Chapnick (2000) defines the top three common factors among the models analyzed by Mosa et al. (2016). Psychological readiness considers "the individual's state of mind as it impacts the outcome of the e-learning initiative," technological skill readiness gauges "observable and measurable technical competencies," and content readiness deals with "the subject matter and goals of the instruction" (Chapnick, 2000, p. 2).

\section{Elearning Success Criteria}

Research shows that the critical success factors of developing and sustaining elearning initiatives include a well-designed and functioning elearning system (Holsapple \& Lee-Post, 2006), social presence in the elearning system (Johnson, Hornik, \& Salas, 2008), institutional and financial support (McGill, Klobas, \& Renzi, 2014), instructors having a positive attitude (Selim, 2007), and development of the faculty and staff (Sife \& Lwoga, 2007). The general problems in developing nations add complexities to elearning not seen in the developed countries, which can inhibit best practices in elearning 
(Bhuasiri et al., 2012). Sife and Lwoga (2007) describe the difficulties of integrating technologies used for elearning in Tanzania and state that "ICTs have not permeated to a great extent in many higher learning institutions in most developing countries due to many socio-economic and technological circumstances" (2007, p. 1). Based on Friesen's (2009) definition of elearning, the role of ICTs is crucial to elearning.

Bhuasiri et al. (2012) evaluate critical success factors for elearning in developing countries, with a focus on ICT experts and faculty. Their study determined six dimensions necessary for implement elearning in developing countries, which are “learners' characteristics, instructors' characteristics, institution and service quality, infrastructure and system quality, course and information quality, and extrinsic motivation" (2012, p. 853). Their study also found that the most important factors "were related to increasing technology awareness and an attitude toward e-learning, enhancing basic technology knowledge and skills, improving learning content, requiring computer training, motivating users to utilize e-learning systems, and requiring a high level of support from the university" (2012, p. 853). Furthermore, their research showed a difference of importance based on the department's perspective. For example, ICT infrastructure and system quality were the faculty's primary concern, while learner characteristics were the most important quality of the ICT team.

\section{Technology Acceptance Model}

This section describes features of TAM and the model's purpose to determine the usage of technology based on user perception. This project uses TAM to determine student perceptions of elearning. 


\section{$\underline{\text { Overview }}$}

The technology acceptance model (TAM) shown in Figure 2 is a systematic approach to testing and explaining the user acceptance of a broad range of new-user information systems (IS) or personal technologies across user populations (Davis, 1989; Davis et al., 1989). Mathieson's (1991) research verifies that TAM is also useful for predicting user intentions to use these technologies. The primary purpose of TAM is "to provide a basis for tracing the impact of external factors on internal beliefs, attitudes, and intention" (Davis et al., 1989, p. 985). Davis et al. argue that researchers can use TAM to determine the aspects of a system that the users find unacceptable and then take corrective action to correct the identified issues. Legris, Ingham, and Collerette (2003) show the variety of technologies where researchers have used TAM, such as voice mail, email, and programming tools. Researchers have also used TAM to investigate user acceptance in education using various web-based elearning systems (Mun \& Hwang, 2003; Ngai et al., 2007; S. Y. Park, 2009; Roca et al., 2006; Wu et al., 2010).

TAM is not without its criticisms or limitations (Christensen, 2013; Chuttur, 2009), but TAM is a highly cited and used model by researchers to determine user acceptance of various technologies (Chuttur, 2009). Chuttur's evaluation of prior research shows that most studies "found significant statistical results for the high influence of perceived usefulness on behavioral intention to use a specific system," and the research "also found mixed results for the direct relationship between perceived ease of use and usage behavior" (2009, p. 13). Studies using the technology acceptance model developed by Davis et al. (1989) showed a limitation of TAM by the lack of explaining the reasons 
why a user perceived the system useful or not, which led Venkatesh and Davis (2000) to extend the model.

TAM is an extensible model that can include external features (Mathieson, Peacock, \& Chin, 2001; Roca et al., 2006). Turner, Kitchenham, Brereton, Charters, and Budgen's (2010) survey of empirical TAM studies confirmed that "many of the studies used modified versions of the TAM rather than the original model” (2010, p. 468). Venkatesh and Davis (2000) created TAM2 by extending the perceived usefulness determinant of TAM to include additional factors that provide granularity to explaining user intention. Venkatesh and Bala (2008) proposed TAM3 to include adding factors to perceived ease of use and incorporate relationships between other determinants. Other researchers have extended TAM with the task-technology fit model (Dishaw \& Strong, 1999; Klopping \& McKinney, 2004).

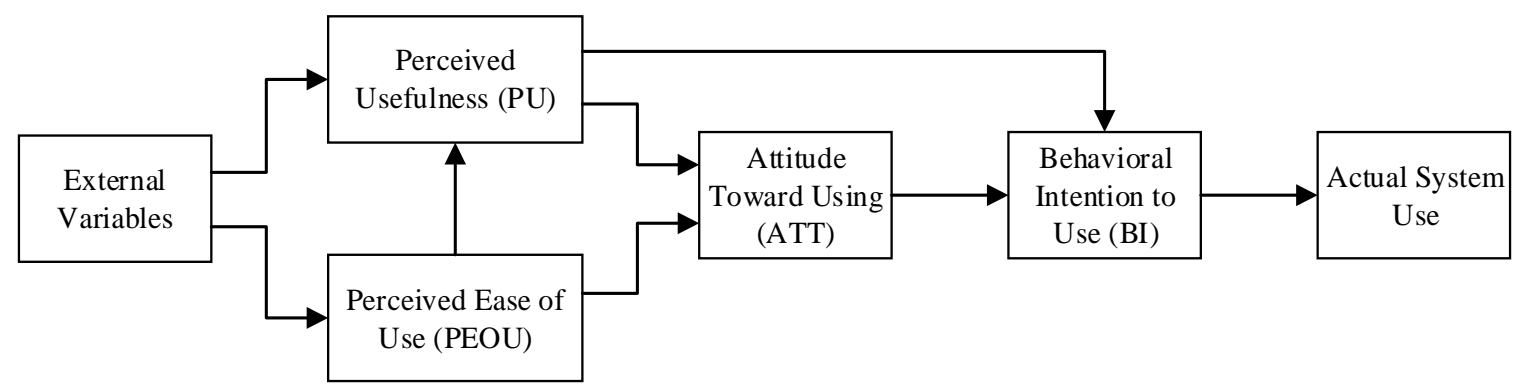

Figure 2: $\quad$ The Technology Acceptance Model (TAM) (Davis et al., 1989)

Theoretical Framework

Foundational elements of TAM that determine actual system use are behavioral intentions (Davis et al., 1989) and attitudes (Davis, 1993). Ajzen and Fishbein (2005) argue that "people's behavior follows reasonably from their beliefs, attitudes, and intentions" (2005, p. 174). A driving factor of behavioral intentions is one's attitude (Montano \& Kasprzyk, 2008). Davis (1993) explains that Ajzen and Fishbein's (1975) 
theory of attitudes is an underlying foundation of TAM, which (a) "specifies how to measure the behavior-relevant component of attitudes," (b) "distinguishes between beliefs and attitudes," and (c) "specifies how external stimuli, such as the objective features of an attitude object, are causally linked to beliefs, attitudes, and behavior" (1993, p. 476).

Davis (1986) derived TAM from the theory of reasoned action (TRA) shown in Figure 3 to evaluate information systems. TRA is a theory of human behavior that focuses "on theoretical constructs concerned with individual motivational factors as determinants of the likelihood of performing a specific behavior" (Montano \& Kasprzyk, 2008, p. 68). They explain that TRA supposes that behavioral intention is the best predictor of behavior, "which in turn is determined by attitude toward the behavior and social normative perceptions regarding it" (2008, p. 68). Davis et al. (1989) revised the proposed version of TAM (Davis Jr, 1986) to incorporate a feature "capable of explaining user behavior" (1989, p. 985). This study refers to Davis' et al. (1989) version as the original TAM, as do other studies (Turner et al., 2010). While both TRA and TAM integrate behavioral attitudes, TAM uses the influences from perceived usefulness and attitude constructs to determine one's behavioral intentions to use the technology. TAM omits subjective norm from the TRA model.

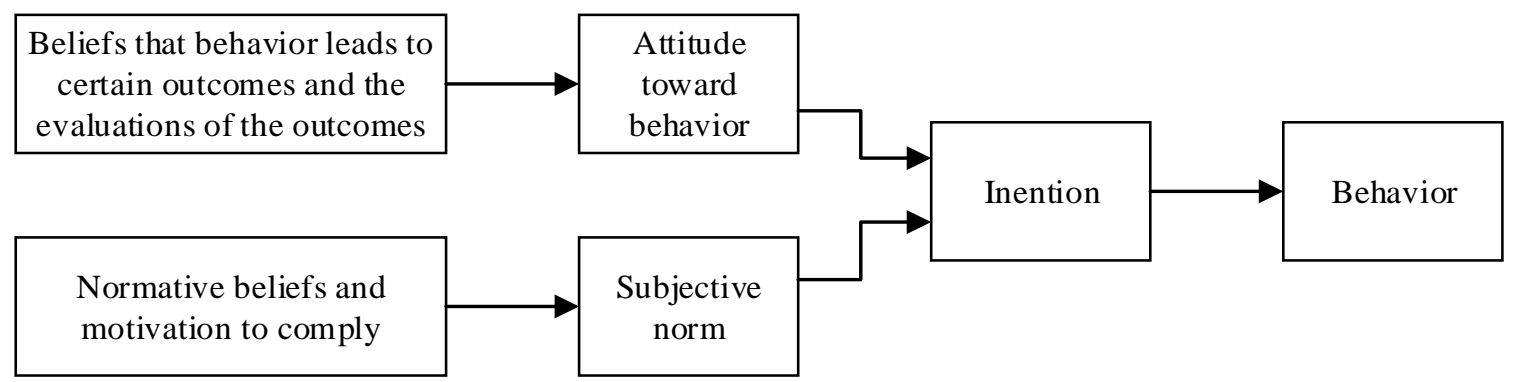

Figure 3: $\quad$ Theory of Reasoned Action (TRA) (Ajzen \& Fishbein, 1980) 
TAM includes the determinants of perceived usefulness (PU), perceived ease of use (PEOU), attitude toward using (ATT), and behavioral intention to use (BI). External factors, such as system design, influence the determinants of PU, PEOU, and BI. Davis (1993) classifies external factors as "external stimulus," PU and PEOU as the "cognitive response," ATT as the "affective response," and actual system use as the "behavioral response" (1993, p. 476). TAM argues "that individuals' behavioral intention to use an IT is determined by two beliefs," which are PU and PEOU (Venkatesh \& Bala, 2008, p. 275). Davis (1989) defines PU as "the degree to which a person believes that using a particular system would enhance his or her job performance" (1989, p. 320). He defines PEOU as "the degree to which a person believes that using a particular system would be free of effort" $(1989$, p. 320).

$\underline{\text { TAM Limitations }}$

Even though researchers frequently cite (Christensen, 2013; Chuttur, 2009) and broadly use TAM in a variety of technology-related fields (Legris et al., 2003), research reveals the limitations of TAM. Known limitations are weak predicting factors, lacking verification of TAM predictions with objective data, using TAM in obligatory contexts, and applying TAM in different cultures. These limitations directly connect to the context of this study.

\section{Examining TAM's Weak Prediction Factors}

Studies show that certain TAM factors are not reliable predictors, notably that PEOU could be a weak predictor (King \& He, 2006; Lee et al., 2005; Turner et al., 2010). Turner et al. (2010) performed a systematic literature review of empirical TAM studies and sought "to determine to what extent the TAM and its revisions have been validated 
for prediction of actual usage" (2010, p. 465). Out of the 79 empirical TAM studies that met their criteria, their results show "that BI is a better predictor of actual usage than either PU or PEU” (2010, p. 470), with PEOU being the worse predictor of actual usage. Turner et al. also note that "All TAM variables are worse predictors of objective usage than subjective usage" (2010, p. 470). Furthermore, their findings indicate that most studies only examined subjective measurements but lacked the objective measurements of actual system usage. They conclude "that it is important to measure actual use objectively as there is a difference in the relationship between the TAM variables and subjective and objective measures of actual technology use" (Turner et al., 2010, p. 471).

King and He (2006) performed a statistical meta-analysis of 88 published TAMbased studies, and their overall conclusion is "that TAM is a powerful and robust predictive model" (2006, p. 751). They note: (a) PU and BI are highly reliable and can be used in a variety of contexts; (b) TAM correlations have considerable variability, but moderator variables can help to explain the effects; and (c) PU profoundly influences BI, which captures much of the effect of PEOU, however, PEOU directly impacts BI in internet applications.

Another limitation described by Turner et al. (2010) is that predicted usage by TAM does not automatically transfer to actual usage. Keung, Jeffery, and Kitchenham (2004) perform a follow-up study of the acceptance of new software cost estimation technology in small software organizations. Keung et al. explain the initial TAM results indicated that users held positive indications about adopting the software. The actual usage results one year after implementing the new software showed almost zero usage. Problems with the software adoption included difficult-to-use software, lack of technical 
support, software that fails to meet the required needs, and the mismatch between the training and company needs (Keung et al., 2004). They argue that "there is a need for more work in the area of early prediction of future usage of a technology" and that TAM might not be "a valid measure for predicting future usage of a technology unless people have experience of using the technology" (2004, p. 58). They recommend assessing the impact of introducing new technology and the plan for implementation (Keung et al., 2004).

\section{$\underline{\text { Using TAM in Obligatory Contexts }}$}

Researchers note that TAM studies primarily focus on the voluntary usage of IS but give little attention to technology acceptance in mandatory environments (S. A. Brown, Massey, Montoya-Weiss, \& Burkman, 2002; Chuttur, 2009). Studies that evaluate using the original TAM in mandatory settings show differences of the user behavioral intention between those in voluntary settings and those in mandatory settings (S. A. Brown et al., 2002; Hartwick \& Barki, 1994). For example, Hartwick and Barki (1994) note a difference between user participation and involvement depending on whether the usage is mandated or voluntary, with the relationship being important only for optional use. In obligatory usage situations, Hartwick and Barki argue that researchers need to locate other success criteria since "the mandatory nature of usage makes it meaningless as an indicant of system success" (1994, p. 454).

S. A. Brown et al.'s (2002) study demonstrated that the relationship between attitude and behavioral intention was not statistically significant. S. A. Brown et al. theorize that in cases of employment, the employees will use the system as long as they remain employed regardless of perceptions. Furthermore, their data showed non- 
significant relationships between the paths of perceived usefulness to behavioral intention and attitude to behavioral intention. To this point, they state that the "Users intend to use the system because they are mandated to do so-perceived behavioural control and subjective norm serve to reinforce this intention" (S. A. Brown et al., 2002, p. 290). Studies on voluntariness in TAM2 and TAM3 show that the subjective norm determinant has a different effect on behavioral intention based on whether the system use is voluntary or mandated (Venkatesh \& Bala, 2008; Venkatesh \& Davis, 2000). Subjective norm is "the degree to which an individual perceives that most people who are important to him think he should or should not use the system" (Venkatesh \& Bala, 2008, p. 277). In TAM2, Venkatesh and Davis (2000) proposed adding the voluntariness construct to address findings from research such as S. A. Brown et al.'s (2002) and Hartwick and Barki's (1994). Venkatesh and Davis's (2000) study included testing four different environments; two included mandatory usage, and two included voluntary usage. Their research revealed that "subjective norm had a direct effect on intentions for mandatory, but not voluntary, usage contexts" (Venkatesh \& Davis, 2000, p. 198), indicating that these two groups exhibit different motivations for their behavioral intentions. Venkatesh and Bala (2008) further tested the voluntariness determinant during the development of TAM3. Their research showed "that the effect of subjective norm on behavioral intention was stronger in a mandatory context" (Venkatesh \& Bala, 2008, p. 290).

Rawstorne, Jayasuriya, and Caputi (2000) describe contexts, such as banking or health care, where using a particular information system is mandatory, and they attempted to predict system usage. In a hospital environment, Rawstorne et al. studied 
three different behavioral intentions on the compulsory use of a patient care information system. These three distinct usages were (a) updating the care plans as changes occurred, (b) using the care plans for planning care delivery, and (c) using the care plans as an educational tool for students and new graduates (Rawstorne et al., 2000, p. 39). Their results showed that TAM failed to predict the behavior of the first usage case, but successfully predicted the usage of the second and third cases.

\section{Using TAM in Different Cultures}

Researchers note the need to explore TAM in different cultures using Hofstede's $(1980,1984)$ cultural dimensions (McCoy et al., 2007; Straub et al., 1997) other than in the North American context where it was developed because predictions are not consistent. For example, Straub et al. (1997) confirm that TAM is suitable for the United States and Switzerland, but not for Japan. Straub et al. note that Japan's cultural tendencies of more uncertainty avoidance and greater power distance are different from those of the other two countries. McCoy et al. (2007) evaluated TAM using respondents from 24 different countries. McCoy et al. tested the significant path coefficients for the TAM relationships of $\mathrm{PU} \rightarrow \mathrm{BI}$, $\mathrm{PEOU} \rightarrow \mathrm{BI}$, and $\mathrm{PEOU} \rightarrow \mathrm{PU}$ according to the four cultural dimensions of uncertainty avoidance (UA), power distance (PD), masculinity/femininity (MF), and individualism/collectivism (IC). They describe the following implications:

Knowing that TAM failed for individuals low in UA could be an important clue that only certain people are sensitive to PU and PEU. Likewise, the failure of key TAM relationships for individuals with high PD, individuals high in Masculinity, and individuals high in Collectivism provides further clues of differences in user sensitivity to TAM constructs. (McCoy et al., 2007, p. 88) 
Conversely, studies show researchers using TAM in diverse cultural contexts, including Africa (I. T. Brown, 2002; N. Park et al., 2009), Asia (S. Y. Park, 2009; T. Teo, 2011), Australia (Rawstorne et al., 2000), Europe (Padilla-MeléNdez et al., 2013; Roca et al., 2006; Šumak et al., 2011), the Middle East (Baker et al., 2010; Tarhini et al., 2013, 2014), and North America (Agarwal \& Karahanna, 2000; Kelly, 2014; Yang \& Yoo, 2004). TAM studies in non-North American contexts range from determining if the model applies to a specific culture to studies that do not mention any cultural implications. The first category is researchers who focus on discovering if TAM applies to a particular context (Al-Gahtani, 2001, 2016; Tarhini et al., 2017). Next are studies that give attention to cultural aspects or confirm that the context is suitable to TAM based on prior research (Alharbi \& Drew, 2014; I. T. Brown, 2002). A third category is those who merely mention limitations of TAM in different cultures (Hsu \& Lu, 2004; T. Teo, 2010; Wu et al., 2010) but operate assuming that TAM produces valid results. The final category of TAM studies in non-North American contexts makes no mention of cultural aspects (Lee et al., 2005; Ngai et al., 2007; N. Park et al., 2009; Šumak et al., 2011).

Examples of using TAM in different cultures include Chen et al. (2017) who used an extended TAM to investigate WeChat gamers in Mainland China for the determinants that affect user intention to engage in mobile social gaming. Ngai et al. (2007) used TAM to examine student acceptance of WebCT in Hong Kong. Using TAM, S. Y. Park (2009) analyzed South Korean students' behavioral intent to use elearning. In Singapore schools, T. Teo (2011) used TAM to evaluate the factors that influence teachers to use technology. N. Park et al. (2009) used TAM to assess user acceptance in developing countries, which included participants in Africa, Asia, and Central and South America. Furthermore, 
researchers have conducted TAM studies on LMSs in Saudi Arabia (Al-Gahtani, 2016; Alharbi \& Drew, 2014).

Some studies that test TAM in cultures different from North America confirm the model's use in that context without modification. One example is Al-Gahtani's (2001) investigation on the applicability of TAM outside of North America using the United Kingdom as the context for the study. Al-Gahtani reports that the results of the research confirmed that TAM is applicable in the UK, and the analysis is consistent with prior research. Principally, Al-Gahtani details that (a) "perceived usefulness is a key intervening variable that links external variables with perceived ease of use, attitudes, and IT acceptance," (b) "perceived ease of use has a strong positive effect on perceived usefulness," and (c) "perceived usefulness has a stronger positive effect on attitudes toward using” (2001, p. 44). Other examples are studies in Lebanon (Tarhini et al., 2013, 2017) that seek to test the moderating factors of the culture by exploring the social norm and quality of work life. Tarhini et al. (2013) conclude that their research "supports the applicability and validity of TAM in an educational context and in the developing countries" (2013, p. 736). Their (2017) study concludes that "TAM is applicable to elearning acceptance within the Arab culture, exemplified here in Lebanon" (2017, pp. $321-322)$.

Some studies show results that are different than expected, such as I. T. Brown's (2002) results which indicate that PEOU has stronger relevance than PU in South Africa. He explains that researchers have extensively studied the PEOU factor in commercial environments or developed countries, but there is a lack of research on PEOU in developing countries. I.T. Brown (2002) investigated the effect of PEOU of web-based 
technologies in a South African learning environment. The participants were first-year university students with limited experience in internet technologies, and the majority were studying in a foreign tongue. I.T. Brown's (2002) conclusions show that PEOU is the dominant factor, outweighing PU. The relevant PEOU features for a web-based learning context where students are functioning in a non-native language are "technology characteristics (ease of finding and ease of understanding) and individual user characteristics (self-efficacy and computer anxiety)" (I. T. Brown, 2002, p. 12). To address the results that "ease of finding and ease of understanding were significant influences on perceived ease of use" (I. T. Brown, 2002, p. 11), the study recommends implementing web-based systems that are easy to navigate. Additionally, the research suggests creating instructional material that uses text that one can easily understand or to provide additional explanations for unfamiliar terms and avoid colloquialisms (I. T. Brown, 2002).

Researchers using TAM with cultural dimensions different from that of North America do not give adequate attention to discussing the impact of culture on TAM as evidenced in Table 7. Fifteen of the thirty-one TAM studies made zero mention of culture or culture-related implications. Four studies had at least one citation to address a cultural issue of the context. Five of the studies included "culture" in the limitations of the study but did not include any citations or specifics. For example, a study in Singapore states that "Such cross-cultural studies have the potential to not only validate the applicability of the TAM in different cultures but add insights to our understanding of technology acceptance by educational users from different cultures" (T. Teo, 2010, p. 77). An investigation in Taiwan concluded that "Other samples from different nations, cultures, 
and contexts should be gathered to confirm and refine the findings of this study" (Wu et al., 2010, p. 163). Additionally, a study from Spain noted limitations to include that "users were from different countries, cultural differences might be reflected in our results, thus, users may vary considerably from culture to culture in satisfaction formation and technology acceptance" (Roca et al., 2006, p. 693).

\section{Table 3: $\quad$ TAM Studies Not Listing Cultural Support}

\begin{tabular}{lll}
\hline \multicolumn{1}{c}{ Study } & \multicolumn{1}{c}{ Context } & \multicolumn{1}{c}{ Cultural Mention } \\
\hline (Keung et al., 2004) & Australia & No mention \\
\hline (Rawstorne et al., 2000) & Australia & No mention \\
\hline (Chen et al., 2017) & China & No mention \\
\hline (N. Park et al., 2009) & Developing world & No mention \\
\hline (Tselios et al., 2011) & Greece & No mention \\
\hline (Lee et al., 2005) & Hong Kong & No mention \\
\hline (Ngai et al., 2007) & Hong Kong & No mention \\
\hline (Shroff et al., 2011) & Hong Kong & No mention \\
\hline (Šmak et al., 2011) & Slovenia & No mention \\
\hline (S. Y. Park, 2009) & South Korea & No mention \\
\hline (Stantchev, Colomo-Palacios, & Spain & No mention \\
Soto-Acosta, \& Misra, 2014) & Taiwan & No mention \\
\hline (Liaw et al., 2007) & Taiwan & No mention \\
\hline (Liaw et al., 2008) & Taiwan & No mention \\
\hline (Lin \& Lu, 2000) & The Netherlands & No mention \\
\hline (Schoonenboom, 2014) & S &
\end{tabular}

Notes: These studies are outside of the North American context. 
Table 4: $\quad$ TAM Studies Listing Cultural Support

\begin{tabular}{|c|c|c|}
\hline Study & Context & Cultural Mention \\
\hline (T. Teo, 2010, 2011) & Singapore & $\begin{array}{l}\text { Inclusion in closing remarks or } \\
\text { limitations with no citation }\end{array}$ \\
\hline (Roca et al., 2006) & Spain & $\begin{array}{l}\text { Inclusion in closing remarks or } \\
\text { limitations with no citation }\end{array}$ \\
\hline (Hsu \& Lu, 2004) & Taiwan & $\begin{array}{l}\text { Inclusion in closing remarks or } \\
\text { limitations with no citation }\end{array}$ \\
\hline (Wu et al., 2010) & Taiwan & $\begin{array}{l}\text { Inclusion in closing remarks or } \\
\text { limitations with no citation }\end{array}$ \\
\hline $\begin{array}{l}\text { (Amer Al-Adwan et al., } \\
\text { 2013) }\end{array}$ & Jordan & $\begin{array}{l}\text { One paragraph describing cultural } \\
\text { limitations with two citations }\end{array}$ \\
\hline (Tarhini et al., 2013) & Lebanon & $\begin{array}{l}\text { The introduction contains references } \\
\text { and information on the need to test } \\
\text { TAM in developing countries. }\end{array}$ \\
\hline (Alharbi \& Drew, 2014) & Saudi Arabia & $\begin{array}{l}\text { Confirmed TAM support for specific } \\
\text { culture }\end{array}$ \\
\hline $\begin{array}{l}\text { (T. S. Teo, Lim, \& Lai, } \\
\text { 1999) }\end{array}$ & Singapore & $\begin{array}{l}\text { Referenced one article pertinent to the } \\
\text { study that relates to TAM and culture }\end{array}$ \\
\hline $\begin{array}{l}\text { (Padilla-MeléNdez et al., } \\
\text { 2013) }\end{array}$ & Spain & $\begin{array}{l}\text { Referenced one article about a cultural } \\
\text { dimension relating to TAM }\end{array}$ \\
\hline (Tarhini et al., 2014) & Lebanon & Inclusion of cultural dimensions \\
\hline (Tarhini et al., 2017) & Lebanon & Inclusion of cultural dimensions \\
\hline (Al-Gahtani, 2016) & Saudi Arabia & Inclusion of cultural dimensions \\
\hline (Baker et al., 2010) & Saudi Arabia & Inclusion of cultural dimensions \\
\hline (I. T. Brown, 2002) & South Africa & Inclusion of cultural dimensions \\
\hline (Al-Gahtani, 2001) & UK & Inclusion of cultural dimensions \\
\hline
\end{tabular}

Notes: These studies are outside of the North American context. 


\section{The Influence of Culture on TAM}

Research indicates that TAM is not a consistent predictor across cultures based on Hofstede's (1980) cultural dimensions. Table 5 shows the results from McCoy et al.'s (2007) study.

Table 5: $\quad$ Expected Significant TAM Paths (McCoy et al., 2007)

\begin{tabular}{|c|c|c|c|}
\hline $\mathrm{PU} \rightarrow \mathrm{BI}$ & $\mathrm{PEOU} \rightarrow \mathrm{BI}$ & $\mathrm{PEOU} \rightarrow \mathrm{PU}$ & Hofstede's (1980) Dimension \\
\hline $\mathrm{No}^{\mathrm{a}}$ & $\mathrm{No}^{\mathrm{a}}$ & Yes & High power distance (PD) \\
\hline Yes & Yes & Yes & Low power distance \\
\hline Yes & $\mathrm{No}^{b}$ & Yes & High collectivism \\
\hline Yes & Yes & Yes & Low collectivism \\
\hline Yes & Yes & Yes & High uncertainty avoidance \\
\hline No & $\mathrm{No}^{\mathrm{c}}$ & No & Low uncertainty avoidance \\
\hline Yes & $\mathrm{No}^{\mathrm{d}}$ & Yes & High masculinity \\
\hline Yes & Yes & Yes & Low masculinity \\
\hline
\end{tabular}

a. Supported by Huang, Lu, \& Wong (2003).

b. Supported by Abbasi, Tarhini, Elyas, and Shah (2015).

c. Supported by Sánchez-Franco, Martínez-López, \& Martín-Velicia (2009).

d. Supported by Srite \& Karahanna (2006).

Huang, Lu, \& Wong (2003) explore the impact of PD in the People's Republic of China. Their operating definition of PD is "the extent to which unequal distribution of power in an organization is accepted by members of the organization" (2003, p. 93).

Their findings show that high PD can negatively influence the PU value of a specific technology. For example, Huang et al. theorize that "an individual's perception of PD in workplaces may render him or her to think that the use of email is not desirable [because] email may reduce reverence to superiors or make superiors feel less authoritative" (Huang et al., 2003, p. 98). The work of Huang et al. (2003) supports McCoy et al. (2007) by showing that PU and PEOU are not accurate predictors of BI in cultures with high PD. Abbasi, Tarhini, Elyas, and Shah (2015) sought to determine the impact of individualism and collectivism on TAM by using participants in the collectivist cultures 
of Pakistan and Turkey. They work under the theory that the belief in PU influences the behavior of persons in individualistic cultures, and conversely, that PEOU is a better predictor of usage for individuals in collectivistic cultures because "decisions to accept something is based on the group's decision" (2015, p. 752). First, they conclude that PU had a strong effect on BI, which "suggests that individuals are likely to accept technology because of the functions it performs (i.e., relative advantages)" (2015, p. 760). Next, they note the insignificance of PEOU on BI and explain that individuals were accepting of the technology because of its functionality regardless of if the technology was hard to use (Abbasi et al., 2015). The work of Abbasi et al. (2015) supports McCoy et al. (2007) by confirming PEOU is not an accurate predictor of BI in cultures high in collectivism.

Sánchez-Franco, Martínez-López, \& Martín-Velicia (2009) evaluated the impact of individualism and uncertainty avoidance on a user's attitude about web-based electronic learning. They used Hofstede's (1991) dimension of uncertainty avoidance, which is "the extent to which the members of a culture feel threatened by uncertain or unknown situations" and work under the assumption that educators in a high uncertainty avoidance culture "are more likely to avoid accepting ICT because of the uncertainty and ambiguity involved" (Sánchez-Franco et al., 2009, p. 591). Their research concludes that PEOU has a negative effect on BI. In practical terms, they state that "uncertainty avoidance societies may not be willing to accept a difficult and irritating interface" (Sánchez-Franco et al., 2009, p. 596), which supports McCoy et al.’s (2007) work that PEOU is not an accurate predictor of BI in cultures with uncertainty avoidance. Contrary to McCoy et al., Sánchez-Franco et al.'s study showed that PU is a strong predictor of BI. 
Srite \& Karahanna (2006) determined the impact of masculinity/femininity values on technology acceptance. They describe individuals with masculine values as those who “emphasize work goals such as earnings, advancement, competitiveness, performance, and assertiveness" (2006, p. 682). Conversely, Srite \& Karahanna describe those with feminine values as tending "to emphasize personal goals such as a friendly atmosphere, comfortable work environment, quality of life, and warm personal relationships" (2006, p. 682). Their findings reveal that "masculinity/femininity values did not moderate the relationship between perceived usefulness and behavioral intention," but moderated "the relationship between perceived ease of use and behavioral intention" (Srite \& Karahanna, 2006, p. 679). These results support McCoy et al. (2007) by confirming that PEOU is not an accurate predictor of BI in high masculine cultures.

\section{$\underline{\text { TAM in Elearning Contexts }}$}

Regardless of the TAM's limitations, educational researchers have used TAM to evaluate the acceptance, satisfaction, or intention to use elearning (Al-Gahtani, 2016; S. Y. Park, 2009; Roca et al., 2006), blended learning (Padilla-MeléNdez et al., 2013; Tselios et al., 2011), e-portfolio systems (Shroff et al., 2011), a digital library system (N. Park et al., 2009), web-based systems (Lee et al., 2005; Mun \& Hwang, 2003), LMSs (Alharbi \& Drew, 2014; Ngai et al., 2007), open education resources (Kelly, 2014), and technology in education (T. Teo, 2011).

In the context of elearning, S. Y. Park (2009) defines PEOU as "the extent to which one believes using e-learning will be free of cognitive effort" (2009, p. 152). External variables such as navigation issues, lack of intuitive design, or unclear instructional materials affect the learner's perceived ease of use. S. Y. Park (2009) 
defines PU as "the extent to which a university student believes using e-learning will boost his or her learning" (2009, p. 152).

S. Y. Park (2009) used TAM to analyze student perception of elearning in fully online courses in a higher education context. He noted issues that inhibit implementing elearning at universities and argues that a significant point is "identifying the critical factors related to user acceptance of technology" (2009, p. 150). He reasoned that researchers should base one area of inquiry on understanding "how students perceive and react to elements of e-learning" (2009, p. 150). His study confirmed that using TAM is "a useful theoretical model in helping to understand and explain behavioral intention to use e-learning" (2009, p. 158). The results showed that the most important TAM construct that affects the students' behavioral intention to use elearning was self-efficacy. The next most important construct was subjective norm, which affects behavioral intention and attitudes. The study notes that PU and PEOU had no direct effect on the students' intention to use elearning, but the determinants influenced their attitudes toward elearning (S. Y. Park, 2009).

Lee et al. (2005) used a modified version of TAM to examine the role of extrinsic and intrinsic motivating factors to determine the student acceptance of an internet-based learning medium (ILM). The extrinsic motivation factors use the PEOU and PU constructs, and the intrinsic motivators used the perceived enjoyment construct. They explain that supporting and improving student learning is a primary goal of using an ILM since "user acceptance and usage are important primary measures of system success" (Lee et al., 2005, p. 1096). Their results showed that perceived usefulness and perceived enjoyment were important factors in affecting student attitude and intention to use ILM. 
Lee et al. (2005) noted that PEOU was not a significant contributor to student attitude, but PEOU indirectly influenced behavioral intention through perceived usefulness and perceived enjoyment.

Mun and Hwang (2003) evaluated student acceptance of Blackboard using TAM.

Like Lee et al. (2005), Mun and Hwang noted that actual system use is a success factor of IS, but explain that student use of Blackboard was voluntary. This project used an extended version of TAM to include the determinants of enjoyment, learning goal orientation, and self-efficacy (Mun \& Hwang, 2003). Their study showed that enjoyment, learning goal orientation, and self-efficacy were relevant factors of user acceptance. Mun \& Hwang (2003) note that ease of use was not a significant determinant.

Ngai et al. (2007) extended TAM to examine student acceptance of WebCT in a higher education context where using the system is mandated. They added a technical support determinant as an external influencer to both PEOU and PU. In evaluating WebCT for both teaching and learning, the goals of their research were threefold: (a) "to determine the current usage of WebCT;" (b) "identify the factors affecting the acceptance of WebCT;" and (c) "develop a model for the acceptance of WebCT in Hong Kong for higher education based on the TAM" (Ngai et al., 2007, p. 251). Their results show that technical support played a significant role in affecting both PEOU and PU. Additionally, their analysis shows a weak direct effect on actual system usage and that "a positive attitude among students towards WebCT may not generate an increase in the actual use of the system if lecturers do not require them to use the system" (Ngai et al., 2007, p. 263). They theorize that this weak connection could be from a compulsory use of WebCT since the students did not have a choice. They confirm the use of TAM in an educational 
context to evaluate LMS usage as they state that their investigation "provides further evidence of the appropriateness of applying TAM to measure the acceptance of WebCT in higher education" (Ngai et al., 2007, p. 263).

\section{$\underline{\text { TAM Dimension Descriptions }}$}

Educational researchers frame the TAM factors in an LMS context to show relevance to the education environment.

Dimensions of PEOU relate to how well the user can navigate the LMS and accomplish his or her intended goal (S. Y. Park, 2009), which includes navigating to the desired module and performing the particular action, such as playing the video or locating the sought after learning materials.

Dimensions of PU relate to items that affect the learner's ability to learn from the LMS (S. Y. Park, 2009). Additional factors such as the quality of content, meaningfulness of interaction with the system, or factors such as the quality of the video and sound influence perceived usefulness. For the learner to find the LMS useful, he or she will need to find equal or more value in using the LMS (S. Y. Park, 2009) as compared to the student's usual way of acquiring knowledge in a traditional learning environment. In other words, the LMS provides the student with more significant learning opportunities than the learner would otherwise have.

Dimensions of ATT relate to the user's positive or negative feeling concerning the use of the LMS, with an intention to act a certain way (Han \& Shin, 2016; McGill \& Klobas, 2009). Examples of emotions relating to the LMS used in prior research are levels of confidence (Liaw et al., 2008) and enjoyment (Ngai et al., 2007; PadillaMeléNdez et al., 2013), the sense of it being a good or bad idea (Alharbi \& Drew, 2014; 
Ngai et al., 2007), and making a positive or negative evaluation (McGill \& Klobas, 2009;

S. Y. Park, 2009; Stantchev et al., 2014).

$\underline{\text { TAM Results from Different Countries }}$

A survey of previous TAM studies in educational and non-educational contexts displayed in Tables 6-8 provides the direct effects of the TAM factors. Table 6 lists studies in educational settings that show the ATT construct. Nine of these thirteen projects show that PU has the highest influence on ATT. Four of the studies illustrate that PEOU has a higher impact on PU than PU does on ATT. Six of the studies reveal that PEOU has the most significant influence, which relates to PU. Tables 6 and 7 are ordered firstly according to studies where PU has the highest direct effect on ATT and then secondly according to the studies where PEOU has the most influence on ATT.

Table 8 shows studies that reported results without the ATT construct, most of which are from non-educational settings. This project refers to TAM without the ATT factor as the simplified TAM model. Nine of these findings illustrate that PU has the highest influence on BI. Four of the studies show that the PEOU factor has the highest impact. 
Table 6: $\quad$ Direct Effects using Educational Studies (Original TAM)

\begin{tabular}{cccccl}
\hline $\begin{array}{c}\text { PEOU } \\
\rightarrow \text { PU }\end{array}$ & $\begin{array}{c}\text { PEOU } \\
\rightarrow \text { ATT }\end{array}$ & $\begin{array}{c}\text { PU } \\
\rightarrow \text { ATT }\end{array}$ & $\begin{array}{c}\text { PU } \\
\rightarrow \text { BI }\end{array}$ & $\begin{array}{c}\text { ATT } \\
\rightarrow \text { BI }\end{array}$ & \multicolumn{1}{c}{ Study and Context } \\
\hline .75 & .06 &. $\mathbf{7 6}$ & - & .93 & $\begin{array}{l}\text { Blended learning; male subjects; Spain } \\
\text { (Padilla-MeléNdez et al., 2013) }\end{array}$ \\
\hline .23 & .37 &. $\mathbf{7 5}$ & - & - & LMS; Hong Kong (Ngai et al., 2007) \\
\hline .47 & .14 &. $\mathbf{6 8}$ & .28 & - & LMS; Slovenia (Šumak et al., 2011) \\
\hline .43 & .34 &. $\mathbf{5 5}$ & - & .62 & $\begin{array}{l}\text { Blended-learning; female subjects; Spain } \\
\text { (Padilla-MeléNdez et al., 2013) }\end{array}$ \\
\hline .12 & .20 &. $\mathbf{5 3}$ & - & .23 & $\begin{array}{l}\text { Elearning; South Korea } \\
\text { (S. Y. Park, 2009) }\end{array}$ \\
\hline .51 & - & $\mathbf{. 5 2}$ & .12 & .53 & $\begin{array}{l}\text { MM Learning System; North America } \\
\text { (Saadé, Nebebe, \& Tan, 2007) }\end{array}$ \\
\hline .48 & .22 &. $\mathbf{5 0}$ & - & .26 & $\begin{array}{l}\text { Students learning to use IS tools; North } \\
\text { America (Yang \& Yoo, 2004) }\end{array}$ \\
\hline .47 & .14 & $\mathbf{. 4 1}$ & - & - & $\begin{array}{l}\text { Technology use in education; Singapore } \\
\text { (T. Teo, 2010) }\end{array}$ \\
\hline .51 & - &. $\mathbf{3 9}$ & .19 & .35 & $\begin{array}{l}\text { Internet-based learning; Hong Kong } \\
\text { (Lee et al., 2005) }\end{array}$ \\
\hline .61 & $\mathbf{. 4 3}$ & .29 & .22 & .48 & $\begin{array}{l}\text { Blended learning; Greece } \\
\text { (Tselios et al., 2011) }\end{array}$ \\
\hline .53 & $\mathbf{. 4 2}$ & .30 & .22 & .50 & $\begin{array}{l}\text { Technology use in education; Singapore } \\
\text { (T. Teo, 2011) }\end{array}$ \\
\hline .504 & $\mathbf{. 3 4 0}$ & - & .265 & $\begin{array}{l}\text { Elearning; Jordan a } \\
\text { (Amer Al-Adwan et al., 2013) }\end{array}$ \\
\hline .71 & $\mathbf{. 3 0}$ & - & - & $\begin{array}{l}\text { E-portfolio; Hong Kong } \\
\text { (Shroff et al., 2011) }\end{array}$ \\
\hline
\end{tabular}

a. Considered a developing nation.

All values are significant at $p<0.05$.

A dash means the results were not reported or not significant.

Ordered by: PU $\rightarrow$ ATT; PEOU $\rightarrow$ ATT. 
Table 7: $\quad$ Direct Effects using non-Educational Studies (Original TAM)

\begin{tabular}{cccccl}
\hline $\begin{array}{c}\text { PEOU } \\
\rightarrow \text { PU }\end{array}$ & $\begin{array}{c}\text { PEOU } \\
\rightarrow \text { ATT }\end{array}$ & $\begin{array}{c}\text { PU } \\
\rightarrow \text { ATT }\end{array}$ & $\begin{array}{c}\text { PU } \\
\rightarrow \text { BI }\end{array}$ & $\begin{array}{c}\text { ATT } \\
\rightarrow \text { BI }\end{array}$ & \multicolumn{1}{c}{ Study and Context } \\
\hline 77 & - & .93 & - & .72 & $\begin{array}{l}\text { Corporate software; North America } \\
\text { (Dishaw \& Strong, 1999) }\end{array}$ \\
\hline .74 & .26 & .47 & .20 & .41 & $\begin{array}{l}\text { Corporate use of a computer with a GUI; } \\
\text { North America } \\
\text { (Agarwal \& Prasad, 1999) }\end{array}$ \\
\hline .23 & .57 & .14 & & .99 & Online games; Taiwan (Hsu \& Lu, 2004) \\
\hline- & .37 & - & .10 & .87 & $\begin{array}{l}\text { Mobile gaming; China (Chen et al., } \\
\text { 2017) a }\end{array}$ \\
\hline .77 & - & .93 & - & .72 & $\begin{array}{l}\text { Corporate software; North America } \\
\text { (Dishaw \& Strong, 1999) }\end{array}$ \\
\hline
\end{tabular}

a. Considered a developing nation.

All values are significant at $p<0.05$.

A dash means the results were not reported or not significant.

Ordered by: PU $\rightarrow$ ATT; PEOU $\rightarrow$ ATT. 
Table 8: $\quad$ Direct Effects (Simplified TAM)

\begin{tabular}{|c|c|c|c|c|}
\hline $\begin{array}{l}\mathrm{PEOU} \\
\rightarrow \mathrm{PU}\end{array}$ & $\begin{array}{r}\mathrm{PEOU} \\
\rightarrow \mathrm{BI} \\
\end{array}$ & $\begin{array}{c}\mathrm{PU} \rightarrow \\
\mathrm{BI}\end{array}$ & $\begin{array}{c}\mathrm{BI} \rightarrow \\
\text { Actual }\end{array}$ & Study and Context \\
\hline .63 & .13 & .65 & - & $\begin{array}{l}\text { Corporate software; North America } \\
\text { (Davis, 1993) }\end{array}$ \\
\hline .19 & - & .64 & - & $\begin{array}{l}\text { Digital library system; Africa, Asia, and } \\
\text { Central/Latin America }{ }^{\text {a }} \text { (N. Park et al., 2009) }\end{array}$ \\
\hline- & .15 & .62 & .38 & $\begin{array}{l}\text { Ecommerce; North America } \\
\text { (Klopping \& McKinney, 2004) }\end{array}$ \\
\hline .30 & .17 & .55 & .52 & $\begin{array}{l}\text { Corporate software; North America } \\
\text { (Venkatesh \& Davis, 2000) }\end{array}$ \\
\hline - & .31 & .48 & - & $\begin{array}{l}\text { WWW; North America } \\
\text { (Agarwal \& Karahanna, 2000) }\end{array}$ \\
\hline- & .22 & .46 & .19 & LMS, North America (Mun \& Hwang, 2003) \\
\hline .21 & .25 & .37 & - & Elearning; Saudi Arabia ${ }^{a}$ (Al-Gahtani, 2016) \\
\hline .38 & .32 & .33 & .35 & $\begin{array}{l}\text { Medical system; Australia } \\
\text { (Rawstorne et al., 2000) }\end{array}$ \\
\hline .35 & .19 & .19 & - & $\begin{array}{l}\text { Internet users; Singapore } \\
\text { (T. S. Teo et al., 1999) }\end{array}$ \\
\hline .63 & .55 & .17 & - & $\begin{array}{l}\text { Banking software; North America (S. A. } \\
\text { Brown et al., 2002) }\end{array}$ \\
\hline .33 & .48 & .38 & .34 & Websites; Taiwan (Lin \& Lu, 2000) \\
\hline .17 & .38 & .24 & - & $\begin{array}{l}\text { Computers in the workplace; Saudi Arabia }{ }^{a} \\
\text { (Baker et al., 2010) }\end{array}$ \\
\hline .81 & - & - & .72 & $\begin{array}{l}\text { Open education resources; North America } \\
\text { (Kelly, 2014) }\end{array}$ \\
\hline
\end{tabular}

a. Context is considered in the developing world.

All values are significant at $p<0.05$.

A dash means the results were not reported or not significant.

Ordered by: PU $\rightarrow$ BI; PEOU $\rightarrow$ BI. 


\section{Learning Management System}

The third major section to discuss is learning management systems because this project uses one as the primary educational tool to facilitate the blended-learning course.

\section{Overview of LMSs}

An LMS is a generalized term for an all-in-one online system that has administrative and learning features to benefit both the educator and the student. Lonn and Teasley (2009) describe an LMS as a collaborative web-based system for instructors and students to share class resources, provide assignment submissions and feedback, and to communicate with each other. This research project uses Watson and Watson's (2007) concept of an LMS with the focus on learning as "the framework that handles all aspects of the learning process" (2007, p. 28). Given the nature of an LMS, one can use it exclusively for administrative work or entirely to support student learning. Educators who use an LMS commonly include both the administrative and learning aspects (Oliveira, Cunha, \& Nakayama, 2016).

Due to the multiplicity of what an LMS encompasses, the term is often misunderstood and misused (Watson \& Watson, 2007). The term LMS is one of several other labels that researchers have coined over the years to define similar activities, but each with their particular focus. For example, Greenberg (2002) argues that the focus of an LMS is for managing learners and tracking their progress, while the emphasis of a learning content management system (LCMS) is on the learning content. Some of the other terms are a course or content management system (CMS), a virtual learning environment (VLE), or an integrated learning system (ILS). 
One could argue that the modern-day LMS is a comprehensive framework that includes many of the unique features of the early systems, such as incorporating advanced course management and learning tools. One example is Blackboard's transition from a course management system to the contemporary LMS. An archived version of Blackboard's website described itself as the "the most widely-adopted course management system among U.S. postsecondary institutions" ("Blackboard Inc.," 2006, para. 3). Watson and Watson's (2007) survey of the early LMS field helped to define the core features of an LMS. Some of these notable features they mention are integration with the university's administrative systems, content creation and delivery, assessments, adherence to technical standards, such as SCORM and AICC, incorporating features of a CMS, customized learning, and creating a learner-centered environment. They argue that the modern-day LMS needs to incorporate constructivist-based instruction, to improve collaborative work, to enhance personalized features for the student that include personalized assessment and adapting to the learner's needs, to become systemic as part of the academic infrastructure, and to improve access to learning resources (Watson \& Watson, 2007).

An LMS is primarily a collection of tools that educators can use to support teaching and learning (Coates, James, \& Baldwin, 2005). These tools allow the user to post announcements, create assignments, share content, upload files, engage in synchronous chat, facilitate asynchronous discussions, display the course schedule, or provide a platform for collaborative work on a wiki (Lonn \& Teasley, 2009). Educators use LMSs in versatile ways to supplement face-to-face classes (Lonn \& Teasley, 2009), create blended-learning courses (Dias \& Diniz, 2014; Georgouli, Skalkidis, \& Guerreiro, 
2008), use as a massively open online course (MOOC) platform (Kop, Fournier, \& Mak, 2011), engage mobile learners (Han \& Shin, 2016), deliver fully online classes (S. Y. Park, 2009), use as personal learning environments (PLE) (García-Peñalvo, Conde, Alier, \& Casany, 2011), or include external "social software to build an interactive and collaborative e-learning platform" (Du, Fu, Zhao, Liu, \& Liu, 2013, p. 18).

Regardless of the versatility of an LMS and its collection of tools, research shows limitations with LMSs to include technical constraints of the platform. These constraints can lead to ineffective use of the available tools to create a student-centered learning environment (García-Peñalvo et al., 2011; Stantchev et al., 2014). One solution is to integrate external tools into the LMS to create a context focused on the student (Conde et al., 2014; Du et al., 2013; García-Peñalvo et al., 2011) or to replace the built-in feature with a cloud-based solution that provides a better learning experience (Stantchev et al., 2014). García-Peñalvo et al. (2011) argue that LMSs need to be open and flexible to adapt to new technologies.

LMS platforms continue to change with ICT and software development practices. Conde et al. (2014) explain that even as development brings changes to web technologies that can enhance student learning, institutions may not adapt to these changes. They list four reasons why educators fail to adopt or actually use these ICT advancements: (a) some educational institutions resist including newer technologies in formal environments; (b) other establishments push these technologies even when they are not required or not the correct solution for the given problem; (c) disparity of digital literacies among students make adapting to these technologies problematic; and (d) educational institutions favor using the LMS for administrative purposes more than using it for student-centered 
learning. Furthermore, ICT changes happen at a pace that LMS vendors are unable to match. One example is the lack of LMS support of web 2.0 tools (Conde et al., 2014). Web 2.0 tools are often complex web applications that the LMS does not need to replicate, nor is it practical to do so. Instead, one can include external web 2.0 applications in the LMS (Conde et al., 2014; Du et al., 2013). Some of these tools, such as Piazza and Twitter, are available through Learning Tools Interoperability (LTI) apps, which directly connect the LMS to the web application to provide functionality without building them into the LMS (Alier, Guerrero, Gonzalez, Penalvo, \& Severance, 2010; Mott, 2010).

$\underline{\text { Prior Educational Uses of LMSs }}$

Educators demonstrate the versatility of LMSs by using them in various ways and with different external tools and instructional designs. Conde et al.'s (2014) pedagogical approach used external web 2.0 applications with Moodle to facilitate a student-centered approach. They used Twitter, Flickr, and WordPress as the web 2.0 tools in the study. Conde et al. (2014) assert that the instructor could assess the student work done in the external applications, rather than doing all of the work in the LMS. Conde et al. used Hootsuite to evaluate the interaction of the students' use of Twitter. These tools allowed Conde et al. (2014) to provide a more authentic learning experience other than was available in the LMS. Conde et al. argue against fully integrating tools such as these since it would change the context and could lessen the learning potential. They recommend that instructors facilitate the use of "external learning activities based on $2.0 \mathrm{Web}$ tools" (Conde et al., 2014, p. 200) to improve student learning. In this type of scenario, Watson 
and Watson's (2007) overview of an LMS holds true since the LMS is the framework that facilitates student learning.

Du et al. (2013) used external tools with an LMS to create an interactive and collaborative elearning platform to facilitate a collaborative working environment. They based their theoretical background on social learning, which combines cognitive, constructive, and social process (Du et al., 2013). They acknowledge the benefits of the traditional approach to elearning using an LMS, but they also recognize the usability gap between the collaborative functionality of built-in tools in the LMS and the functionality of external web 2.0 tools. They propose creating a hybrid LMS that combines collaborative and social functionality with the existing capabilities of the LMS. Their model includes also creating a personalized space for the user to collaborate and interact with others to share, tag, or comment on resources (Du et al., 2013).

Georgouli et al. (2008) explore a learner-centered method for using an LMS in a traditional course to create a blended-learning course. They enhanced traditional pedagogical approaches "to deliver the educational material, to activate existing knowledge, to produce and apply new knowledge, to support the community and to motivate the students" (2008, p. 238). Georgouli et al. argue that educators can apply the model across various fields, but the adopters need to consider what type of blended learning approach to integrate. They assert that the term blended learning describes "a learning format that combines several different delivery methods" and "learning that mixes various event-based activities, such as face-to-face classrooms, live e-learning, and self-paced learning" (Georgouli et al., 2008, p. 229). Their theoretical model uses LMS features that include components for administration, community, content, and activities 
that designers can incorporate into their course to support enhanced learning. They assert that the administrative component is critical to the model, but the other three provide opportunities for the instructor to build in specific learning activities relating to the selected component. Georgouli et al. argue that the model supports students and improves their quality of learning (2008).

\section{Choosing an LMS}

There is a variety of LMSs for institutions to select based on specific organizational or methodological requirements. According to edutechnica, four LMS vendors account for over $80 \%$ of the usage based on the list of educational institutes that edutechnica monitors (“Spring 2017 updates,” 2017). These top LMSs are Blackboard Learn (33\%), Instructure Canvas (19.8\%), Moodle (18.9\%), and D2L Brightspace (10\%). Each of the companies that sponsor these four LMSs offer their products as a software as a service (SaaS). Instructure Canvas and Moodle provide open source versions of their LMSs for an organization to self-host the LMS, but Blackboard and D2L do not offer this option. Edutechnica only evaluates the organizations that use SaaS subscriptions and do not self-host the open-source LMSs.

Cavus (2013) explains the factors that an institution in a developing country should consider when choosing an LMS. Cavus notes the hidden costs, localization limitations, bandwidth requirements, and integration problems with a university's current system. She recommends that these institutions use an open source LMS, such as Moodle, due to their quality and feature sets that rival their commercial counterpart (Cavus, 2013). Her research included 92 participants with prior LMS knowledge and 23 without LMS experience. Their LSM evaluation tool, a specially designed computer 
application that the instructors used to evaluate an LMS, included 52 features of an LMS and a list of 42 LMSs. The participants would select an LMS based on their criteria and then use it for four weeks. The LMSs that the instructors ranked the highest or found the most suitable for their needs were Moodle (39\%), WebCT (27\%), and Blackboard (27\%) (Cavus, 2013).

Oliveira et al. (2016) discuss the criteria that an institution should consider when selecting an LMS. The categories of LMSs that they examine are commercial, proprietary, or free LMSs. They list the LMS evaluation criteria in categories of "reliability, scalability, security, sustainability, and adoption of international standards of quality" (Oliveira et al., 2016, p. 161). They highlight several LMSs available on the global market including Blackboard and Moodle, which indicate that the LMS supports localization. Additionally, they stress that choosing an LMS depends on the institution's goals, and not necessarily on the LMS with the best feature set (Oliveira et al., 2016). $\underline{\text { Moodle as an LMS }}$

Moodle is a commonly used LMS because it contains an adequate set of features, is open-source, and allows for different pedagogical approaches. Al-Ajlan and Zedan (2008) evaluated the features, capabilities, and technical aspects of ten commonly used LMSs to determine the LMS that ranks the highest, which is their criteria for selecting an LMS. Their first LMS comparison based on learning, pedagogical, and administrative features showed Moodle ranking among the top of the LMSs. Their second study compared the ten LMSs for their technical features, which revealed that Moodle outperformed all other LMSs. Al-Ajlan and Zedan note that Moodle ranked high due to its rich feature set. They conclude that Moodle is the overall best choice for an LMS (Al- 
Ajlan \& Zedan, 2008). This study provides relevance for showing a comparison of LMS features, but it has some notable limitations. First, an organization should not choose an LMS based on the most abundant set of features (Oliveira et al., 2016). Additionally, more features increase system complexity, which can decrease usability (Friedman, 1996).

Al-Ajlan and Zedan's (2008) study also include reasons to choose Moodle and its limitations. The reasons to choose Moodle include: (a) Moodle is free to install and use because of its open source licensing; (b) it contains a standard feature set that instructors need for teaching online or in a blended format; (c) Moodle uses common server-side programming languages and technologies that many servers support without modifications, which simplifies the installation process; (d) developers created Moodle with pedagogy and technology in mind; (e) Moodle is widely used internationally; and (f) it has broad support from documentation and the online communities (Al-Ajlan \& Zedan, 2008). The limitations of Moodle that Al-Ajlan and Zedan found primarily relate to the nature of open source software, which affects the institutions that choose to self-host Moodle. The list includes: (a) Moodle requires an IT specialist to configure and maintain efficiently; (b) it requires a course administrator to oversee the administrative features of Moodle; and (c) Moodle lacks an official support channel, which requires users with problems to post help to the forums (Al-Ajlan \& Zedan, 2008).

Kop et al. (2011) used Moodle as the LMS platform for a massively open online course (MOOC) to explore how new technologies influence the design of learning environments. Examples are changes that occur from a traditional learning context to blended learning or to a fully online context. Each context can change, such as an LMS 
moving from a resource center to an active learning environment. Kop et al. argue that these learning environments need to be agile to adapt to these emerging technologies. While Moodle was not the focus of their study, they showed that Moodle is capable of pedagogical adaptations that result from changes in the learning environment (Kop et al., 2011).

Horvat, Dobrota, Krsmanovic, \& Cudanov (2015) evaluate the student perceptions of the quality and satisfaction of students' learning experiences using the Moodle LMS. The study took place at a Serbian state university and included 395 participants. This study does not accurately evaluate Moodle independently of teaching methodologies; instead, a focus of Horvat et al. (2015) is on teaching quality, which assesses the quality of instructional material, student interaction, and assignment feedback. In other words, this study evaluates the quality of the learning experience of a course taught using Moodle, and not an evaluation of Moodle independent of these external factors. However, this study is still relevant since it shows that students have a favorable perception of using Moodle in a blended learning format in a non-Western country. Horvat et al. (2015) report notable findings that student perception correlated with system use. They explain that students who use Moodle infrequently, such as only before an exam, had a lower satisfaction of the system as compared to those who frequently used Moodle (Horvat et al., 2015).

\section{Data Analytics in Learning Management Systems}

A final component of this project to discuss is collecting objective data from Moodle to confirm the TAM perception data. The two methods mentioned in this section are educational data mining $(\mathrm{EDM})$ and learning analytics (LA). EDM is the process of 
applying statistical, machine learning, and data mining to educational contexts (Romero \& Ventura, 2010, 2013). While EDM focuses on methods of exploring, collecting, and transforming the data, learning analytics (LA) is "the measurement, collection, analysis, and reporting of data about learners and their contexts" (Luna, Castro, \& Romero, 2017, p. 90). There are similarities and overlap between LA and EDM, but LA applies known predictive models to focus on the description of data and results (Romero \& Ventura, 2013). Luna et al. (2017) state the objective of LA "is to understand and optimise the learning process and the environments in which it occurs" (2017, p. 90).

One platform that researchers apply EDM to is LMSs (Romero, Cerezo, Bogarín, \& Sánchez-Santillán, 2016; Romero, Ventura, \& García, 2008). Romero et al. (2016) explain that an LMS stores student data, interactions, and other usage data in files and databases. Researchers can then mine this data and transform it into logical datasets that they can analyze. One of the core goals of EDM "is to make this valuable data legible and usable to students as feedback, to professors as assessment, or to universities for strategy" (Romero et al., 2016, p. 3). An example of EDM is mining LMS data that LA can use to alert instructors to at-risk students, with the hope that the educator can provide timely intervention (Macfadyen \& Dawson, 2010).

An application of EDM is learning analytics dashboard applications, which display the data in a usable or graphical format that benefits the user (Verbert, Duval, Klerkx, Govaerts, \& Santos, 2013). These dashboards are grouped into three categories, which are ones that support face-to-face lectures, face-to-face group work, and blended or online learning courses (Verbert et al., 2014). The dashboard could provide a historical picture of a student's progress, activities, and show performance indicators that the 
learner can then adjust to meet learning goals (Verbert et al., 2013). Verbert et al. explain that teachers can also benefit from dashboards to get a picture of how the overall class is performing or how individual students are progressing, which assists teachers in identifying areas in which learners need additional help.

$\underline{\text { Data Mining and Moodle }}$

Data mining and learning analytics are well researched using Moodle data as an integrated solution or as an external solution using exported data (Luna et al., 2017; Romero et al., 2016; Romero, Espejo, Zafra, Romero, \& Ventura, 2013; Romero et al., 2008; Romero \& Ventura, 2010). Romero et al.'s (2008) foundational case study of data mining in Moodle provides a detailed description and a step-by-step look at how others can apply the techniques. Data mining techniques that one can apply to Moodle data include statistics, visualization, clustering, classification, and association rule mining (Romero et al., 2008). Romero et al. (2013) apply data mining to elearning systems to predict final exam grades of students in a Moodle course. For this study, they developed a Moodle mining tool to extract and organize the data. One development goal for the application was to make it usable by both non-expert and expert users (Romero et al., 2013). Romero et al. (2016) explore EDM in the context of Moodle datasets. Moodle datasets provide abundant data since Moodle logs detailed behavioral and user data, such as mouse clicks that are used for system functionality (Romero et al., 2016). Researchers can use these same data for EDM and LA.

Luna et al. (2017) evaluate and discuss the benefits of learning analytics dashboards in Moodle. They explain that Moodle's default reporting tools extract the basic data and allow the user to filter it, but the data are primarily in raw form and require 
analyzing and interpreting. Furthermore, they note that the current LA tools in Moodle could only provide a general overview and state that these tools "are unable to discover new, interesting, and useful knowledge" (Luna et al., 2017, p. 91). As a result of these limitations, Luna et al. developed a data mining tool specifically for Moodle, called the Moodle Data Mining Tool (MDM), “to extract interesting and previously unknown knowledge hidden in Moodle data by means of different DM techniques" (Luna et al., 2017, p. 91).

There are freely available LA dashboards for use within Moodle that improve on Moodle's standard interface (Luna et al., 2017; Verbert et al., 2013). When Luna et al. (2017) created MDM, they also surveyed a broad range of other LA tools. Some analytic tools are free or integrate into Moodle, while others are or do not. The free LA tools identified by Luna et al. (2017) that Moodle can incorporate are GISMO, SNAPP, MOClog, SmartKlass, MEAP, and Analytics graphs. Verbert et al. (2013, 2014) evaluate learning dashboards, of which only Moodle Dashboard and SNAPP match the list by Luna et al. (2017).

Researchers can also use LMS system data to verify the predictions of TAM studies (Mun \& Hwang, 2003; Turner et al., 2010). While educators can "analyze the students' course activities and usage information to get a general view of a student's learning” (Romero \& Ventura, 2010, p. 604), researchers can use the same data to compare the behavioral intention construct to actual system use. This area of verification of TAM lacks research since the majority of studies do not collect this data, but primarily determine a theoretical actual usage value based on perception data (Turner et al., 2010). 


\section{CHAPTER FOUR: METHODOLOGY}

\section{Introduction}

Chapter four describes the research methodology and procedures used to answer the research objectives, which were to determine if the technology acceptance model (TAM) is an appropriate method for determining student readiness for elearning in Kazakhstan using the factors of perceived usefulness (PU), perceived ease of use (PEOU), attitude toward using (ATT), and behavioral intention to use (BI). This surveyresearch based project collected data from a state university in Kazakhstan and then performed a path analysis to build a TAM profile. These results were then compared to TAM studies in the developed world. Besides providing student perception data about elearning in the research context, the implications of this research also impact other TAM-based studies in various cultures that are similar to Kazakhstan. Due to the success of using TAM to predict student usage of elearning technology in developed countries (Liaw et al., 2007; Ngai et al., 2007; S. Y. Park, 2009), researchers in the developing world also apply the model without giving adequate consideration to how different cultural aspects impact TAM (Chen et al., 2017; N. Park et al., 2009).

The section on data collection describes where the data were collected, the research participants, and the instrumentation and procedures for collection. The data analysis section describes the processes of how the data were analyzed and validated. Chapter four also includes instrument validity and reliability, ethical considerations, bias, and limitations. 


\section{Statement of the Problem and Research Questions}

A crucial success factor in adopting elearning methodologies is student readiness, but there is a lack of research in the less economically developed countries. Furthermore, research shows that using TAM in Kazakhstan might not be reliable because of Kazakhstan's high power distance, collective, and masculine culture (Abbasi et al., 2015; Huang et al., 2003; McCoy et al., 2007; Srite \& Karahanna, 2006). The purpose of this study was to use the original version of TAM to determine if the factors affecting student readiness to engage in elearning in Kazakhstan were the same factors as those identified in developed countries. Specifically, the methodology described how the project addressed the research questions of:

1. Does PU have a stronger influence than PEOU on ATT in Kazakhstan?

2. How do the significant factors of PU, PEOU, ATT, and BI in Kazakhstan compare to the significant factors in developed countries?

\section{Research Methodology}

The quantitative method for this project used survey research to outline the data collection procedures (Creswell, 2014; Ruel et al., 2015). The data analysis used principal component analysis (PCA) component extraction methods (Abdi \& Williams, 2010; O’Rourke \& Hatcher, 2013; Smith, 2002; Wold, Esbensen, \& Geladi, 1987) and path analysis (Duncan, 1966; Garson, 2008) to identify the underlying relationships between variables that affect student perception of a learning management system (LMS).

The literature demonstrates that researchers can use survey research to collect data for LMS (Ngai et al., 2007; Oliveira et al., 2016; S. Y. Park, 2009) and TAM studies (S. A. Brown et al., 2002; Davis, 1989; Lee et al., 2005). Researchers also use survey 
research to analyze TAM data on LMSs (Alharbi \& Drew, 2014; Liaw et al., 2008; Ngai et al., 2007).

A survey of prior studies shows that researchers widely use path analysis to analyze TAM (Agarwal \& Prasad, 1999; Dishaw \& Strong, 1999; Klopping \& McKinney, 2004; Lin \& Lu, 2000; N. Park et al., 2009; Rawstorne et al., 2000; T. S. Teo et al., 1999). Other studies demonstrate that PCA and path analysis are used together to analyze TAM (Chen et al., 2017; Liaw et al., 2008; T. Teo, 2010). PCA is a component extraction and reduction method (Bro, Kjeldahl, Smilde, \& Kiers, 2008; Jolliffe, 2002; Vyas \& Kumaranayake, 2006; Williams, Onsman, \& Brown, 2010) that researchers use with factor analysis and path analysis research methods (Choi \& Baek, 2011; Ngai et al., 2007; Padilla-MeléNdez et al., 2013).

Principal Component Analysis (PCA)

Principal component analysis is primarily a variable reduction technique to extract the constructs or principal components (PC) that show the number of underlying components or structure (Jolliffe, 2002; O'Rourke \& Hatcher, 2013; Smith, 2002; Vyas \& Kumaranayake, 2006; Wold et al., 1987). Jolliffe \& Cadima (2016) argue that researchers of various disciplines use PCA, and it is "one of the oldest and most widely used" (2016, p. 2) methods to reduce a dataset to dimensions. There are many variations and adaptations of PCA, but its primary uses remain descriptive and not inferential (Jolliffe, 2002; Jolliffe \& Cadima, 2016). PCA is useful if there is redundancy or correlation among variables (O'Rourke \& Hatcher, 2013; D. D. Suhr, 2005) since PCA creates new uncorrelated variables to maximize the variance and increase the interpretability of the data (Jolliffe \& Cadima, 2016). PCA shares procedural similarities 
with factor analysis, but researchers stress that the statistical method of PCA is not factor analysis (Jolliffe, 2002; O’Rourke \& Hatcher, 2013; D. D. Suhr, 2005) and argue that they "are really quite distinct techniques" (Jolliffe, 2002, p. 150). Wold et al. (1987) describe the goals of PCA as data simplification, data reduction, modeling, outlier detection, variable selection, classification, prediction, and unmixing. Smith (2002) describes an advantage of PCA as its ability to compress the data without losing much information through the component reduction process.

\section{$\underline{\text { Path Analysis }}$}

Path analysis uses multiple regression to describe a result of a model based on the direct and indirect effects of a set of variables (Garson, 2008). Researchers consider path analysis to be an appropriate statistical method for analyzing TAM data because it can "test theoretical models that specify directional relationships among a number of observed variables" (O’Rourke \& Hatcher, 2013, p. 108) with the focus on interpretation, and not on discovering causes (Duncan, 1966). Liaw et al. (2008) state that "path analysis is an appropriate multivariate analytical methodology for empirically examining sets of relationships in the form of linear causal models" (2008, p. 958). Additionally, Keith (2014) argues that "path analysis makes many aspects of multiple regression more understandable, and it is often a better choice for the explanatory analysis of nonexperimental data" (2014, p. 243).

Path analysis is a special case of structural equation modeling (SEM) with several notable differences. One difference from other multivariate statistical analysis methods is that path analysis requires specifying a model (D. Suhr, 2008). Another difference from 
SEM is that path analysis only contains manifest variables with one indicator each, which does not reveal cross-factor influences (Garson, 2008).

O'Rourke \& Hatcher (2013) list the necessary conditions for path analysis, which are: (a) using interval or ratio-level measurements, (b) minimal of five continuous endogenous values, (c) normally distributed data, (d), linear and additive relationships between variables, (e) absence of multicollinearity, (f) absence of measurement error, (g) inclusion of all nontrivial causes, and (h) use an overidentified model. An overidentified model is one that includes more known than unknown elements (O'Rourke \& Hatcher, 2013).

\section{$\underline{\text { Sample Size Justification }}$}

Researchers do not agree on the sample size for SEM analysis but use rules of thumb or recommendations (O’Rourke \& Hatcher, 2013; Westland, 2010) with some researchers looking at ratios and others looking at the total sample size (Osborne \& Costello, 2004). O'Rourke and Hatcher (2013) argue that a stable analysis requires at least 100 participants, and Kline (2015) recommends a sample-size-to-parameter ratio of 20:1. In addition to having an ideal sample size, researchers argue that SEM analysis should have a sample size of at least a ratio of 10:1 (Bentler \& Chou, 1987; Kline, 2015; Osborne \& Costello, 2004; D. Suhr, 2008). They argue that smaller sample sizes, such as $5: 1$, also reduce the trustworthiness of the results or produce unstable estimates.

\section{Learning Management System}

This project used Moodle for the LMS due to its popularity, simplified installation procedure, comprehensive online support, multi-language support (Al-Ajlan \& Zedan, 2008), data mining options and support (Luna et al., 2017), favorable student perceptions 
(Horvat et al., 2015; Šumak et al., 2011), and pedagogical support for this project (AlAjlan \& Zedan, 2008; Kop et al., 2011).

\section{Research Design}

The research design used blended learning to teach information communication technology (ICT) to first-year students studying at a university in Kazakhstan. The subjective data were collected from the participants using a pretest and two posttests. The pretest provided insights into the instrument and problematic question. The two posttests attempted to fine-tune the instrument and determine reliability. Objective data were collected from an analytical tool that anonymously recorded the participants' behavior. PCA was used to extract components from the survey data for use by path analysis. The components were applied to TAM using path analysis to determine the direct and indirect impact of each TAM factor.

\section{Elearning Methodology}

This project utilized Moodle, as the LMS, to incorporate course materials using a pedagogical approach in the face-to-face freshmen ICT class. The instructional methodology of the course was a flipped classroom (O'Flaherty \& Phillips, 2015; Tucker, 2012). Most of the class happened inside the LMS platform, with face-to-face meetings being secondary. The five specific components of interest in the LMS for this study were (a) video lectures with subtitles and transcripts, (b) embedded Google Slide presentations with speaker notes, (c) laboratory assignments, (d) announcements, and (e) grades. Due to the limited number of students who had adequate skills to study in English, the principal researcher taught only one of the ICT course sections using Moodle to facilitate a blended-learning environment. Other ICT instructors used Moodle with a guest login so 
that their students could access the materials in English, which included online laboratory assignments, presentations, and videos.

Based on the syllabus, the ICT course contained 15 modules that correspond with each week of the semester. These 15 modules in Moodle provided the student with a single access point to the logically organized content that supports ease of use. The videos for the in-class lectures included English subtitles and a transcript. The students could view the embedded Google Slide presentations and read the speaker notes, which were also the video transcripts. The assignments section contained details on how the students could complete and submit their required work, which included labs and group projects. The online forums provided a place for the students to ask questions and serve as a communication point for the instructor (Lonn \& Teasley, 2009). The grades in Moodle provided feedback to students about the state of their work. These features benefited the students by enabling ease of access to course material, an opportunity to replay lecture videos, a way to view the presentation and read the speaker notes, an open communication channel for help, access to the instructor, and a single place for announcements and course news.

\section{Data Description}

\section{$\underline{\text { TAM Dimensions }}$}

Perceived usefulness (PU) is the extent to which one believes using an LMS will boost his or her learning (S. Y. Park, 2009). Perceived ease of use (PEOU) is the extent to which one believes using an LMS will be free of cognitive effort (S. Y. Park, 2009). Attitudes toward using (ATT) is one's positive or negative feeling toward the LMS 
(Montano \& Kasprzyk, 2008). Behavioral intention (BI) is the degree to which one intends to use an LMS (Davis et al., 1989).

\section{$\underline{\text { LMS Component Factors }}$}

The LMS components were not included in an extended TAM model because of low sample size. Instead, these external factors were used to describe PU as a latent variable.

Course video lectures (CVL) were voice-over PowerPoint presentations that contained the material that the instructor typically gives during a lecture. This component related to both technology and content. PU of CVL sought to determine if the students found the learning materials beneficial in video format.

Course online presentations (COP) were the Google Slide versions of the PowerPoints used for the video lectures. They also included the speaker notes so that the students could read the content while viewing the slide. PU of COP sought to determine if the students found the learning materials beneficial.

Course laboratory assignment (CLA) provided an online version of the ICT laboratory projects and submission details. This component related to both technology and content. PU of CLA sought to determine if the students found the online version of the assignments beneficial.

Course announcements forums (CAF) were a place for instructor-to-student communication. The instructor could post announcements or course-related information. This component related to technology and the learner readiness factors. PU of CAF sought to determine if the students found the forums beneficial. 
Course online grades (COG) provided the students with feedback and grades on submitted assignments and other course activities. The instructor provided online grades and feedback using text or uploaded documents to student-submitted work. This component related to technology and the learner readiness factors. PU of COG sought to determine if the students find the grades and feedback beneficial.

\section{Principal Component Analysis}

This project used PCA to reduce variables and extract components from the survey data for use with path analysis. This project followed D. D. Suhr's (2005) procedure for conducting variable reduction using PCA, which included:

1. performing the initial PCA, which extracts the number of components that equal the number of observed variables

2. determining the number of components to retain using the eigenvalue $>1$ criterion, scree test, verifying the proportion of variance for each component and cumulative proportion of variance explained, and determining interpretability

3. performing rotations

4. interpreting rotated solutions $(2005$, p. 4$)$

\section{$\underline{\text { Path Analysis }}$}

This project performed a path analysis on TAM using the extracted component to show the influence of the PU, PEOU, ATT, and BI factors that direct actual system use. This project followed D. Suhr's (2008) suggested approach to path analysis: 
1. review the relevant theory and research literature to support model specification

2. specify a model (e.g., diagram, equations)

3. determine model identification (e.g., if unique values can be found for parameter estimation and if the number of degrees of freedom (df) for model testing is positive)

4. select measures for the variables represented in the model

5. collect data

6. conduct preliminary descriptive statistical analysis (e.g., scaling, missing data, collinearity issues, outlier detection)

7. estimate parameters in the model

8. assess model fit

9. respecify the model if meaningful

10. interpret and present results. (2008, p. 3)

\section{Population and Sample}

The sample came from first-year students enrolled in the required ICT classes that used an LMS as part of the course. The 2017-2018 freshmen student body population was approximately 800 students. Out of the 800 students, 47 students enrolled in the researcher-led ICT course that used an LMS as the primary educational tool. Another 5075 students used the LMS to access the ICT laboratory studies and instructional content in English. These students were typical university students from the area. There were no unique characteristics of the participant population. The university did not have a gender bias, so the student body included a standard ratio of male and female students. The 
ethnicities primarily included Kazakh and Russian students. The first language of most of the students was Kazakh or Russian, with English being a foreign language.

The legal age of consent in Kazakhstan is 16 years old. Kazakhstan traditionally has had 11 years of compulsory education, instead of the standard 12 years in the US system. Their education system results in students routinely entering university at age 17 . The university considers all their students as consenting adults. This project's IRB application followed the university's recommendation and treated 17-year-olds as consenting adults.

The first-year students at the university were not accustomed to technologysupported learning and were not prepared for studying in a digital environment. Aspects of unprepared students for elearning are their lack of understanding the time required for elearning, critical thinking skills, and general academic competencies (Parkes et al., 2015). Unprepared students are accustomed to a systemic way of education that resembles the traditional learning model (Gilbert, Morton, \& Rowley, 2007). Instead, elearning expects the students to interact with the system, learning material, and other learners in different sequences (Gilbert et al., 2007). Student preparedness for elearning also depends on their motivation and technical competencies (Selim, 2007).

Another characteristic was that students had a range of technical skills. Those from the outlying areas would be proficient on a smartphone, but possibly have low exposure to a desktop or laptop computer. Overall, the first-year students had a limited level of English. The results of the English placement tests based on the Common European Framework of Reference for Languages (CEFR) scale for the 800 freshmen students showed that $6.25 \%$ were B1 (upper intermediate level) and only $0.5 \%$ were B2 
(advanced level). The university estimates that students need at least a B1 level of English to study the ICT course in English. Furthermore, it was likely that this ICT course was the students' first experience with a foreign instructor.

\section{Instrumentation}

This project collected and analyzed two different sets of data, which were subjective and objective data. The subjective dataset was the perception survey data based on TAM. The objective dataset was the anonymous analytical data from the LMS that showed how the students used the elearning system. The two datasets painted a picture of how the users perceived the LMS and how they actually used it. The data used for the TAM analysis were collected using pretest and posttest surveys that contained questions relating to the participant's perception data of the LMS. The anonymous LMS usage that showed how the students used the elearning system helped to interpret the perception data.

\section{$\underline{\text { Survey Instrument }}$}

The survey contained demographics, four primary factors that directly correspond to the TAM components, five external factors relating to the perceived usefulness of the blended course, and readiness factors selected from Chapnick's (2000) readiness model (See Appendix A). The essential part of the survey that addressed the research questions were the four TAM factors of PU, PEOU, ATT, and BI. The other survey questions provided clarity, helped to explain the results, or benefited to the local educational context. Each component that used PCA analysis met the criteria of at least three questions per component, but this project sought to include the recommended number of five questions per component (O’Rourke \& Hatcher, 2013). 
The demographics (DEM) revealed basic information about the audience, gained insights about their previous elearning experience (PEX), and sought to determine their technology skill (TEC). The readiness factors sought to establish the participants' technology readiness (TR), learner readiness (LR), and content readiness (CR) for engaging in elearning. The external perceived usefulness components sought to add clarity to the PU construct, which were video resources (PU-VR), online presentations (PU-PR), ICT laboratory studies (PU-LAB), course announcements (PU-AN), and online grades (PU-OG). The PU element provided a general perception of Moodle, but it did not provide insight into the student responses.

This project did not include perceived ease of use data for the external TAM factors because the expected TAM results for this audience was that PEOU does not significantly influence BI (Abbasi et al., 2015; Huang et al., 2003; McCoy et al., 2007; Srite \& Karahanna, 2006). Instead of focusing on what was not expected to work and determining which aspects were difficult to use, this project was more interested in discovering what specific elements the students found useful from a practical standpoint. By establishing which elearning components the students found useful, the university could make an informed decision about the direction of their elearning initiative. They could also focus on ease of use once usefulness and component selection had been established.

\section{$\underline{\text { Web Analytics }}$}

This project used Matomo, a web analytics application to capture the behavioral data of the LMS users anonymously. The analytics program showed the number of visits to the LMS, what pages they visited the most frequently, when they used the LMS, and 
how much time they spent on the page. The objective data provided insights into how the students were using Moodle and helped to validate the results of the perception-based surveys.

\section{Data Management and Collection}

This project used Qualtrics, a web-based survey application, to electronically administer the pre- and two posttest surveys. The researcher placed a link to the survey on the ICT course page in Moodle. The ICT instructors who used Moodle asked their students to participate in the anonymous survey. The participants were informed of their privacy, that taking the survey was optional, and that there was no consequence or reward for their choice to participate. The data collection period for each survey round lasted approximately two weeks, with most students participating during the first week. The anonymous survey data was downloaded from Qualtrics using a secure connection and then stored in an encrypted cloud computing system. The identifiable features in the survey data, such as student cohorts, were coded to protect the privacy of the participants. The data was stored in an encrypted 7-Zip file once all data were analyzed.

The behavior data was collected automatically and anonymously as the students used the LMS. The university informed the student that they would be participating in an elearning research project. Data access to Moodle and Matomo used a forced-SSL connection. The web analytical data was downloaded and stored in an encrypted 7-Zip file. 


\section{Data Analysis and Procedures}

\section{$\underline{\text { Preliminary Analysis }}$}

The initial data analysis examined the results of unrotated factor loading and correlation matrices, and it tested whether the dataset was factorable and suitable for PCA and path analysis. The correlation matrices provide an overview of the correlations to determine that the data support component extraction (Tabachnick \& Fidell, 2007), with the correlation values being at least $>0.3$ to meet the minimal threshold (Williams et al., 2010). The anti-image correlation matrix diagonals should produce values $>0.5$ to determine the factorability of the data (Kaiser \& Rice, 1974). KMO is another method to determine if the data are suitable for PCA process (Williams et al., 2010). The KMO and Bartlett's test should produce a KMO value greater than 0.6 (Kaiser, 1974). The communality estimates was another method used to validate the data, "which represent the common variance of each variable analyzed" (Ford, MacCallum, \& Tait, 1986, p. 293). Variables with coefficients outside of the low-threshold mark of 0.4 (Costello \& Osborne, 2005) could be excluded.

\section{Principal Component Analysis}

The data analysis included the precursory step of PCA to extract the principal components (Smith, 2002; Vyas \& Kumaranayake, 2006; Wold et al., 1987).

Furthermore, the PCA procedure included cross-validation to assist with the selection of the components to eliminate variables that correlated with another factor (Abdi \& Williams, 2010; Bro et al., 2008; Josse \& Husson, 2012; Wold, 1978).

The analysis followed the PCA procedure detailed by O'Rourke and Hatcher (2013). The first part of the process was to perform the initial PC extraction and 
determine which components to keep based on four criteria. The first criterion was the eigenvalue-one criterion, which retained the extracted components with eigenvalues $>1$. The next criterion to determine the number of factors was to examine the scree plot of the eigenvalues and keep the components before the break. O'Rourke and Hatcher explain that the researchers should retain those values on the graph before the break, which explains the largest amount of variance. The third criterion was calculating the variance accounted for by the selected components. O'Rourke and Hatcher (2013) recommend retaining the components that account for $5 \%$ or $10 \%$ of the total variance, with the retained factors accounting for 70\%-80\% of the total variance. Lastly, the interpretability criteria provided guidelines how to interpret "the substantive meaning of the retained factors and verifying that this interpretation 'makes sense' regarding what is known about the constructs under investigation" (O'Rourke \& Hatcher, 2013, p. 26). The next chapter details the PCA extraction procedure and documents the extracted variables below one eigenvalue.

The second part of the PCA procedure was performing a rotation based on factor loading (O'Rourke \& Hatcher, 2013) that then allowed for using a cross-validation technique to remove misplaced variables (Abdi \& Williams, 2010). For example, the rotation showed that a variable from component 1 correlates better with component 3 , and therefore should be removed. The two main types of rotations are orthogonal and oblique (Abdi \& Williams, 2010). Orthogonal rotations are uncorrelated whereas oblique rotations permit correlation among factors. In social sciences, researchers recommend using varimax rotation or orthogonal rotation (Abdi \& Williams, 2010; O’Rourke \& Hatcher, 2013). One standard cross-validation method that can be used is called jackknife 
(Efron, 1982), also known as leave-one-out (Abdi \& Williams, 2010; Bro et al., 2008). This project used oblique rotation because the TAM assumes correlation among factors. $\underline{\text { Path Analysis }}$

The first step to analyzing the TAM data using path analysis was to determine any significant correlations between the PEOU and PU variables. Then, regressions were run on each path to determine the coefficients and explained variance. Figure 4 shows the paths. The TAM paths are (a) PEOU $\rightarrow$ PU, (b) PEOU $\rightarrow$ ATT, (c) PU $\rightarrow$ ATT, (d) PU $\rightarrow$ BI, and (e) ATT $\rightarrow$ BI. The final step was to assess the model fit (O'Rourke \& Hatcher, 2013; D. Suhr, 2008). Assessing model fit included reviewing the $\mathrm{r}^{2}$ values of endogenous variables and significance tests for path coefficients and covariances (O’Rourke \& Hatcher, 2013).

The final step to analyzing the TAM data was to determine if the actual system used corroborated BI by comparing behavioral data from the LMS to the perception data. Turner et al. (2010) found that the majority of TAM studies relied on the perception data to determine actual system use, but Turner et al. recommend validating actual system use prediction with objective data collected from the LMS. Since the students were expected to use the LMS, the real system data becomes imperative because prior research indicates that users exhibit different behavioral intentions in mandatory environments (S. A. Brown et al., 2002; Hartwick \& Barki, 1994). 


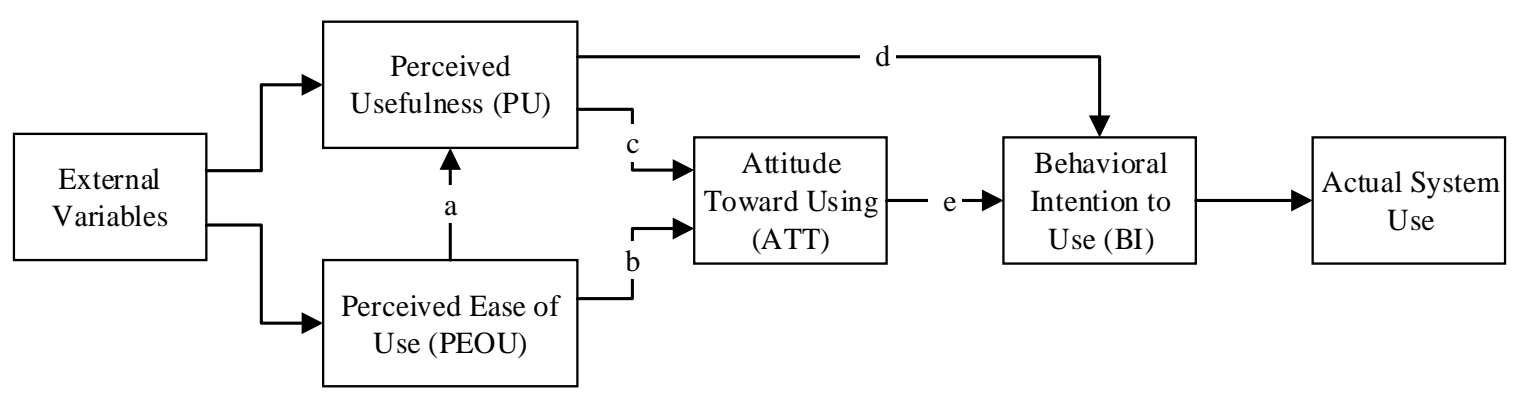

Figure 4: $\quad$ TAM with Regression Paths

\section{$\underline{\text { Analyzing External Factors }}$}

By design, all variables in a path analysis or manifest variable model are manifest or observable variables (Garson, 2008; O’Rourke \& Hatcher, 2013). Conversely, a latent variable model contains observable variables that inform a hypothetical construct or latent variable about the amount of influence of each manifest variable (O'Rourke \& Hatcher, 2013). Because this project did not include the external TAM variable in the path analysis model due to the sample size, PU became a latent variable to infer the influence of each external factor. Figure 5 shows the effect of indicators on perceived usefulness.

This project used the objective data from the LMS to help interpret the subjective data (Romero \& Ventura, 2010; Turner et al., 2010). Student-use data from Moodle was captured using Matomo, a web analytics application. Matomo’s dashboard provided variations of the aggregated data in tables or charts. The visual inspection of the overview identified high-level patterns of student use. The pertinent data included how frequently the student logged on, which sections of the LMS they visited most often, and which devices they used to view Moodle. The behavioral data of how the students actually used Moodle (i.e., what they found useful) provided insight into their perception data (i.e., 
how they wanted to find it useful). This data helped bring understanding to anomalies or results that needed clarity.

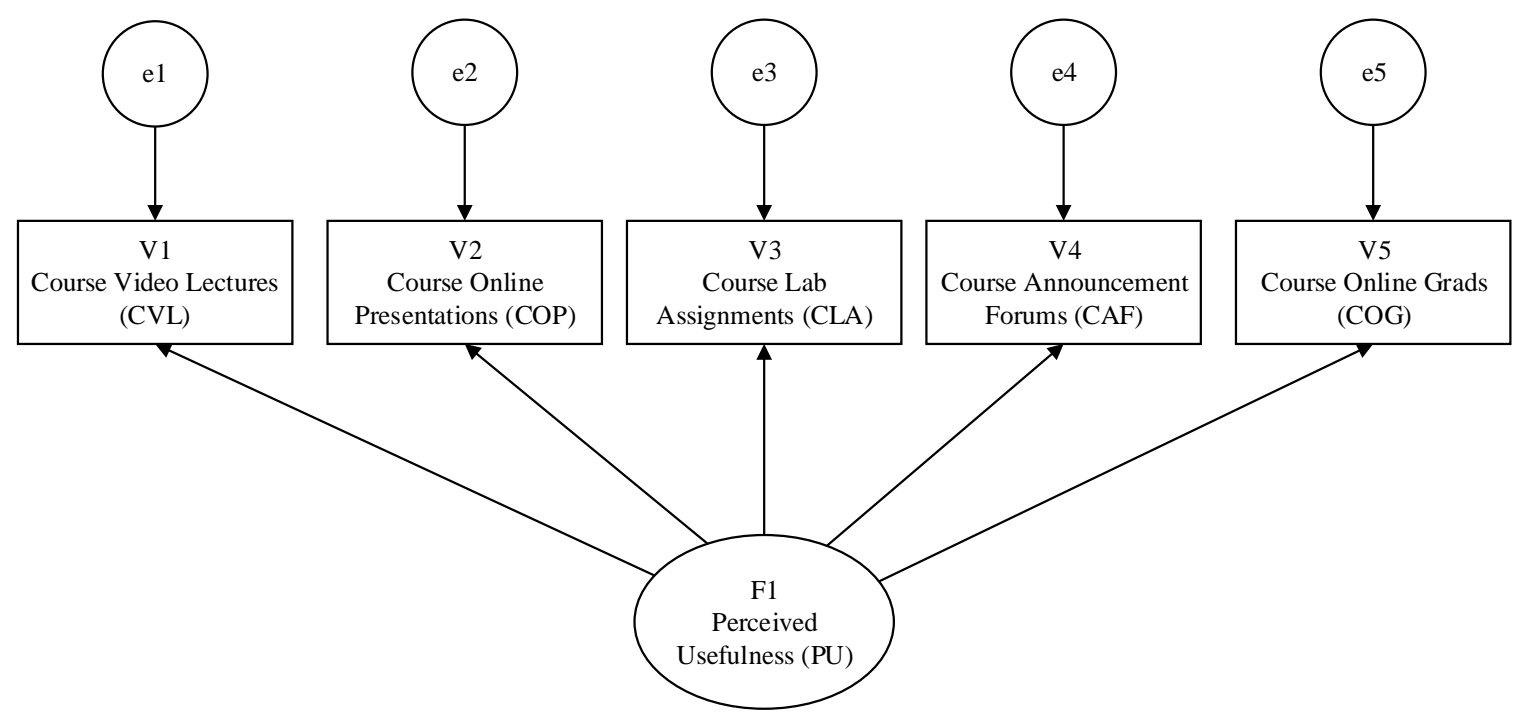

Figure 5: $\quad$ Latent Variable Model for PU

$\underline{\text { Answering Research Questions }}$

This project used a qualitative approach to answer the two research questions listed in Table 9. Both questions used the results from this project's path analysis. See Figure 4 for path information. TAM is a widely used model with known cultural limitations. This project explored using a qualitative comparison to determine if TAM in Kazakhstan produced similar results to studies in developed countries. The first comparison evaluated a fundamental assumption about TAM that PU has a more significant effect than PEOU on BI (King \& He, 2006). Therefore, we can know that one aspect of TAM is comparable to other studies if this supposition is satisfied in Kazakhstan. The next comparison evaluated a broader view of TAM to determine if the significant TAM paths in Kazakhstan were equivalent to studies in developed nations. The studies listed in Tables 6 and 7 show that some paths are significant less frequently 
than other paths. A failed comparison could indicate that the model in this study is different from other models.

Table 9: $\quad$ Alignment of Research Questions to Data Analysis

\begin{tabular}{|c|c|c|}
\hline Research Question & Data & Data Analysis \\
\hline $\begin{array}{l}\text { 1. Does PU have a stronger } \\
\text { influence than PEOU on } \\
\text { ATT in Kazakhstan? }\end{array}$ & Survey data & $\begin{array}{l}\text { PCA + Path analysis; } \\
\text { Measured path values b and c; } \\
\text { Determined if } c>b\end{array}$ \\
\hline $\begin{array}{l}\text { 2. How do the significant } \\
\text { factors of PU, PEOU, ATT, } \\
\text { and BI in Kazakhstan } \\
\text { compare to the significant } \\
\text { factors in developed } \\
\text { countries? }\end{array}$ & $\begin{array}{l}\text { Survey data; } \\
\text { Results from } \\
\text { other TAM } \\
\text { studies }\end{array}$ & $\begin{array}{l}\text { PCA + Path analysis; } \\
\text { Qualitatively compared findings from } \\
\text { this research context to findings from } \\
\text { other TAM studies: } \\
\text { - Was path } \mathrm{x} \text { significant at } p<0.05 \text { ? } \\
\text { - If not, should it be significant? }\end{array}$ \\
\hline
\end{tabular}

The first question used a greater-than comparison on the path analysis results to determine if the direct influence of PU on ATT (path c) was greater than the direct influence of PEOU on ATT (path b). Therefore, the question would be satisfied if path c $>$ b.

Answering the second research question used a subjective comparison to determine if the TAM factors in Kazakhstan were the same as in developed countries using two criteria. The first criterion revealed if the path was significant. Next was to evaluate if the path should be significant. Even though this project assumed that in developed countries all TAM paths were significant at $p<0.05$, a successful TAM analysis does not require all paths to be significant, but specific paths should be significant. King and He (2006) argue that researchers are more likely to publish their studies that show significant results than studies that lack significance. Additionally, their meta-analysis research revealed that path PEOU $\rightarrow$ BI was insignificant at the 0.05 level 
in 30 out of 67 studies. Prior research shown in Tables 6-8 revealed that TAM paths were not always significant due to unexpected factors or the data accurately showed a lack of technology acceptance. However, the data demonstrate that some paths are significant frequently, such as PEOU $\rightarrow$ BI (King \& He, 2006) when evaluating the simplified TAM.

\section{Validity and Reliability}

The first step to addressing validity and reliability issues was to base the survey questions on prior research (Ruel et al., 2015). The researcher derived the survey questions from previous TAM, TAM3, and LMS studies, but changed the context of the items to reflect the elements of this project (See Appendix A). Prior research influenced PU (Agarwal \& Prasad, 1999; Alharbi \& Drew, 2014; Chen et al., 2017; Legris et al., 2003; McGill \& Klobas, 2009; Ngai et al., 2007; Padilla-MeléNdez et al., 2013; S. Y. Park, 2009; Roca et al., 2006), PEOU (Ngai et al., 2007; S. Y. Park, 2009; Roca et al., 2006; T. Teo, 2011; T. S. Teo et al., 1999; Turner et al., 2010), and ATT questions (Agarwal \& Prasad, 1999; Alharbi \& Drew, 2014; Chen et al., 2017; Ngai et al., 2007; Padilla-MeléNdez et al., 2013; S. Y. Park, 2009; Šumak et al., 2011; T. Teo, 2011). PU, PEOU, ATT, and BI questions all use a seven-point Likert-type scale, from $1=$ strongly disagree to 7 = strongly agree based on previous TAM studies (S. A. Brown et al., 2002; Lee et al., 2005; McGill \& Klobas, 2009; Ngai et al., 2007; S. Y. Park, 2009).

Ruel et al. (2015) recommend validating the survey instrument and measurements using a pretest. The pretest was administered after the students had used the LMS for four weeks to identify issues in the respondents' comprehension of the questions, pinpoint problem areas, and look for signs that question order influences a particular response 
(Ruel et al., 2015). The second survey round was conducted during week ten to evaluate the changes to the survey. The final round of data was collected using a posttest during week fifteen, just before the end of the semester. The participants took the surveys electronically using Qualtrics.

Researchers recommend using a version of the survey in the students' native language to reduce their chance of misunderstanding the questions (Fink, 2016; McKay et al., 1996; Ruel et al., 2015). Given the multilingual context, translators created a Russian version for the students using the back-translation method. Russian was selected since it is the lingua franca of the area. Translating surveys are complex activities that must take into account language and culture (Fink, 2016; McKay et al., 1996; Ruel et al., 2015). To alleviate translation problems, researchers recommend planning for the translation process, using a pair of professional translators knowledgeable of the culture, and then back-translating the survey to the original language to identify inconsistencies of the translation (Fink, 2016; Ruel et al., 2015). McKay et al. (1996) explain that backtranslation is a three-step process using multiple translators. First, a translator translates the survey from the original language to the target language. Another qualified translator then translates the target language back to the original. The final step is to compare the back-translated copy and the original to ensure the accuracy of the translation. (McKay et al., 1996).

\section{Ethical Considerations}

This project followed Creswell's (2014), Ruel et al.'s (2015), and the Boise State University (BSU) Social \& Behavioral Institutional Review Board's (SB-IRB) ethical practices of survey research during the data collection and analysis phases to protect the 
participants. SR-IRB issued protocol number 104-SB17-216 on 11/30/2017 indicating that the project protocol complied with BSU's Federal Wide Assurance (\#0000097) and the DHHS Regulations for the Protection of Human Subjects (45 CFR 46).

Creswell (2014) explains that points of concern for ethical issues in survey research happen at distinct points, which include collecting data, analyzing results, and reporting results. Two ethical issues related to electronically gathering data are maintaining confidentiality and anonymity (Creswell, 2014; Ruel et al., 2015). The university informed the students that they were participating in a research project, which included their behavioral data on the LMS and the survey results, and that the researcher would ensure that the student data remained anonymous. The survey included a consent form that informed the students that their responses were anonymous and that their answers would not identify the respondent. The survey maintained anonymity and did not intentionally contain features that point to the participant (Creswell, 2014).

The raw analytical data from the LMS might contain codes that identify a user. After downloading the analytical data from the LMS at the end of the semester, any data fields that included identifiable participant codes were assigned a unique identifier for data aggregation. No record of the link between the unique identifier and the participant's codes was kept. Therefore, no student identity was revealed. Any uncoded data downloaded from the LMS was stored in an encrypted 7-Zip file until it was destroyed at the end of the project. Another ethical practice is to accurately report the findings of the data and note biases that might skew the data (Ruel et al., 2015), which this project reported in the analysis and discussion chapters. 


\section{Role of the Researcher and Addressing Biases}

The area most prone to researcher bias was during the data analysis phase. There were several areas of concern. First was deciding which survey responses were invalid when cleaning the data. If the path analysis results were not stable, the investigator then sought to remove careless entries consistently. For example, some participants entered the same value for the PU, PEOU, ATT, and BI questions. These responses were removed if a student entered the same value for the perception responses in the survey, such as strongly agree or strongly disagree.

Next, was to determine which factors to include in the path analysis based on the PCA results. Removing a single component could significantly alter the impact of a path. The researcher gave attention to looking at the components that produced stable results over desirable results. Presumably, one could massage the data to produce an anticipated result or omit unfavorable responses.

Third, and perhaps most importantly, was the decision to include all three surveys in this report instead of choosing a posttest to represent the population. The inconsistency among the results changed the outcomes of this project. The analysis centered on the inconsistent data among the survey rounds instead of exploring external factors that might have informed a participant's decision.

\section{Limitations and Delimitations}

One notable limitation of this study's methodology was the small sample size. Due to a limited number of participants, the data could not reliably support an extended TAM analysis to determine the impact of external factors on PU. Furthermore, this 
project did not evaluate the factors that influenced the results. Instead, this study only reported the findings and did not infer about the influences.

Another limitation was the exclusion of the PEOU construct in the extended TAM. This project favored PU over PEOU because of practical implications of wanting to provide useful tools over those that were easy to use but might not be as useful.

A third limitation of this study was the lack of statistical analysis on the behavioral data. Instead, this study subjectively compared how the participants used the elearning platform to the results of the behavioral intention construct in TAM. Arguably, statistically analyzing and comparing with the objective data could reveal insights into the data discrepancies.

A fourth limitation was the studies selected for the comparative analysis. A more extensive amount of TAM studies or data from meta-analysis research should be used for future studies to provide additional data support for how TAM typically behaves in different cultures.

Based on these limitations, additional research is needed to evaluate the external factors that significantly influence the TAM components of PEOU and PU from both the perception and behavioral dataset.

\section{Summary}

This project used survey research, PCA, and path analysis to evaluate the viability of using TAM in Kazakhstan to predict elearning usage because Kazakhstan has cultural dimensions different from North America where the TAM was developed. The research was conducted using first-year students enrolled in the required ICT course. The students 
used Moodle to engage in elearning by watching lecture videos, viewing presentations, and completing ICT laboratory assignments.

The project collected and evaluated subjective and objective data. The perception data came from three survey rounds, which were a pretest and two posttests. The survey data was analyzed using PCA and path analysis to determine the path weights for TAM. The elearning system collected behavioral data, which was used to validate the effect of behavior intention construction on TAM and to help interpret or clarify the results.

Table 9 shows the data analysis used to answer the research questions. The analysis for question one used a greater-than comparison on TAM paths PU $\rightarrow$ ATT and PEOU $\rightarrow$ ATT. Answering research question 2 used a subjective comparison to determine if the TAM factors in Kazakhstan are the same as in developed countries using two criteria. The first criterion determined if the path was significant. Next was to evaluate if the path should be significant because prior research shown in Tables 6-8 revealed that some paths are statistically significant less frequently than other paths.

A limitation of the project was the small sample size due to the limited number of students who met the minimum threshold to study in the ICT course section taught in English. The small sample limited the data analysis to the four TAM constructs of PEOU, PU, ATT, and BI. Another limiting factor was the lack of statistical analysis of the external factors that could clarify the PEOU and PU responses. 


\section{CHAPTER FIVE: DATA ANALYSIS AND RESULTS}

\section{Introduction}

The purpose of this study was to determine if the significant technology acceptance model (TAM) factors in developed countries are the same factors as those in Kazakhstan. The underlying objective was to assess whether TAM is a suitable method for predicting usage of elearning systems in Kazakhstan. The quantitative approach for this project utilized survey research, principal components analysis (PCA), and path analysis (PA) to build a TAM profile using participants from Kazakhstan that was then used for the qualitative comparison. This project used comparative analysis to answer the research questions, which subjectively compared the TAM results in Kazakhstan to results of TAM studies in developed countries.

This chapter is divided into several sections describing the participants, discussing the overview of the findings, illustrating the principal component and path analyses, and explaining the comparative analysis. This overview of the findings provides a summary of the results of the survey questions. The interpretation and detailed explanation of the questions are in chapter six.

The demographics describe the population and explain inconsistencies in the three samples. The PCA analysis section demonstrates the component extraction procedures and data validation procedures. The path analysis segment describes the data in the form of the TAM models, showing both the original and simplified TAM versions. Finally, the 
comparative analysis describes the procedure of examining the results of TAM studies in developed countries and comparing them to the results of this study.

This project utilized a pretest and two posttests to collect and validate the data (See Appendix A). The paper refers to these surveys as Survey I, Survey II, and Survey III. This project lists the data from all these surveys because the TAM analysis revealed that some aspects were consistent while other parts were inconsistent. Given the inconsistent behavior of TAM in Kazakhstan, conclusions were not drawn from any single set of data. This project applied the data from each of the three surveys to the original TAM and the simplified TAM models to test the robustness of TAM, which resulted in six different analyses. This project included the simplified TAM model to provide an equal comparison to the work of McCoy et al. (2007) since they used the same model to evaluate cultural dimensions and TAM. To restate, the original TAM model includes the four factors of perceived usefulness (PU), perceived ease of use (PEOU), attitude toward using (ATT), and behavioral intention to use (BI) (Davis et al., 1989). The simplified TAM model omits the ATT construct and uses the three factors of PU, PEOU, and BI (Venkatesh \& Bala, 2008; Venkatesh \& Davis, 1996).

\section{Participants}

Survey I resulted in 51 usable responses and Survey II contained 80 valid answers. The demographics between the two groups varied. Survey I contained more students who were male, preferred using Russian, and had a higher level of English. Survey II resulted in more female students who prefer Kazakh and had an overall lower level of English. Part of the disbalance comes from the participation of Cohort 8682 in the Survey II that accounted for $25 \%$ of the responses, but the cohort members did not 
participate in Survey I (Tables 11 and 12). This cohort was two-thirds female, all were preferred Kazakh speakers, and only one participant had an English level greater than A2 (intermediate). Survey III suffered from low-quality survey responses, which resulted in excluding $23 \%$ of the 56 responses. The low-quality responses where those with the same response, which included seven entries of strongly agree, five of agree, and one with neither agree nor disagree. Given the varied demographics and inaccuracies, this section lists all three survey results for comparison (See Appendix A).

The data were cleaned before performing the analysis. All incomplete responses were removed. Entries were removed where the participants entered the same value for each TAM question because these entries lacked validity. It is not known if these respondents thought the same about each question or if they entered the data carelessly. For example, some participants answered strongly agree for all questions while others answered strongly disagree to all options.

\section{$\underline{\text { Gender and Language (DEM) }}$}

Table 10 shows the overview of the demographics based on gender, language preference, and level of English. Survey I had a higher percentage of males, Russian speakers, and better English. Survey II contained slightly more females, a preference towards Kazakh, and lower English. Survey III had participants with similar gender and language preference. The polls did not elicit information about the students' ethnicity. Instead, the language of instruction was extracted from the cohort information because that is how the university placed students in groups. Consequently, the language preference of the student does not always follow ethnicity. In general, a Kazakh-preferred speaker is probably an ethnic Kazakh. However, some ethnic Kazakhs prefer to study in 
Russian. Conversely, an ethnic Russian or minority will most likely favor Russian even if he or she is bilingual.

Each survey had varied participants from different cohorts. Table 11 shows the demographics of the top six groups. Cohort 8682 did not participate in Survey I, but they made up at least $23 \%$ of the participants for Surveys II and III. Table 12 shows the makeup of Cohort 8682 that participated in Surveys II and III. This group studied in Kazakh, and at least $65 \%$ of the participating members were female. However, Cohort 8682 had lower English. These are some of the reasons why Survey I data are skewed towards more male participants and Russian language preference. Surveys II and III have more female and Kazakh-speaking participants.

Table 10: $\quad$ Demographics (General) DEM1-DEM4

\begin{tabular}{lcccccc}
\hline & \multicolumn{2}{c}{ Survey I } & \multicolumn{2}{c}{ Survey II } & \multicolumn{2}{c}{ Survey III } \\
& $\mathrm{N}$ & $\%$ & $\mathrm{~N}$ & $\%$ & $\mathrm{~N}$ & $\%$ \\
\hline Gender & & & & & & \\
$\quad$ Male & 36 & $70.6 \%$ & 34 & $42.5 \%$ & 21 & $48.8 \%$ \\
$\quad$ Female & 14 & $27.5 \%$ & 45 & $56.3 \%$ & 22 & $51.2 \%$ \\
Preferred Language of Instruction & & & & & & \\
$\quad$ Russian & 38 & $74.5 \%$ & 33 & $41.3 \%$ & 21 & $48.8 \%$ \\
Kazakh & 13 & $25.5 \%$ & 47 & $58.8 \%$ & 22 & $51.2 \%$ \\
Level of English & & & & & & \\
$\quad$ A1 & 7 & $13.7 \%$ & 15 & $18.8 \%$ & 14 & $32.6 \%$ \\
A2 & 13 & $25.5 \%$ & 26 & $32.5 \%$ & 7 & $16.3 \%$ \\
B1 & 27 & $52.9 \%$ & 31 & $38.8 \%$ & 16 & $37.2 \%$ \\
B2 or above & 4 & $7.8 \%$ & 8 & $10.1 \%$ & 6 & $13.9 \%$ \\
\hline
\end{tabular}


Table 11: Top 6 Cohorts Participation Rate

\begin{tabular}{cccccccccccc}
\hline & \multicolumn{3}{c}{ Survey I } & \multicolumn{4}{c}{ Survey II } & \multicolumn{3}{c}{ Survey III } \\
Cohort & $\mathrm{N}$ & $\%^{\mathrm{a}}$ & $\% \mathrm{C}^{\mathrm{b}}$ & Cohort & $\mathrm{N}$ & $\%^{\mathrm{a}}$ & $\% \mathrm{C}^{\mathrm{b}}$ & Cohort & $\mathrm{N}$ & $\%^{\mathrm{a}}$ & $\% \mathrm{C}^{\mathrm{b}}$ \\
\hline 8477 & 9 & 17.6 & 17.6 & 8682 & 20 & 25 & 25 & 8682 & 10 & 23.3 & 23.3 \\
8684 & 6 & 11.8 & 29.4 & 6582 & 10 & 12.5 & 37.5 & 8484 & 7 & 16.3 & 39.6 \\
6582 & 5 & 9.8 & 39.2 & 8684 & 6 & 7.5 & 45 & 8684 & 4 & 9.3 & 48.9 \\
7784 & 4 & 7.8 & 47 & 8482 & 5 & 6.3 & 51.3 & 7784 & 4 & 9.3 & 58.2 \\
7168 & 4 & 7.8 & 54.8 & 6620 & 5 & 6.3 & 57.6 & 7668 & 3 & 7 & 65.2 \\
7383 & 3 & 5.9 & 60.7 & 8383 & 4 & 5 & 62.6 & 6685 & 3 & 7 & 72.2 \\
\hline
\end{tabular}

a. Percent of sample.

b. Cumulative percentage.

Table 12: Top Cohort Specifics

\begin{tabular}{lcccccccc}
\hline & \multicolumn{2}{c}{$8682^{\mathrm{b}}$} & \multicolumn{2}{c}{$6582^{\mathrm{b}}$} & \multicolumn{3}{c}{$8477^{\mathrm{a}}$} & \multicolumn{2}{c}{$8684^{\mathrm{a}}$} \\
& $\mathrm{N}$ & $\%$ & $\mathrm{~N}$ & $\%$ & $\mathrm{~N}$ & $\%$ & $\mathrm{~N}$ & $\%$ \\
\hline Gender & & & & & & & & \\
$\quad$ Male & 7 & $35 \%$ & 3 & $30 \%$ & 6 & $66.7 \%$ & 6 & $100 \%$ \\
$\quad$ Female & 13 & $65 \%$ & 7 & $70 \%$ & 2 & $22.2 \%$ & 0 & \\
$\quad$ Not reported & - & & - & & 1 & $11.1 \%$ & - & \\
\hline Preferred Language of & & & & & & & & \\
Instruction & & & & & & & & \\
$\quad$ Russian & 0 & 0 & 7 & $70 \%$ & 7 & $77.8 \%$ & 4 & $66.7 \%$ \\
$\quad$ Kazakh & 20 & 100 & 3 & $30 \%$ & 2 & $22.2 \%$ & 2 & $33.3 \%$ \\
\hline Level of English & & & & & & & & \\
$\quad$ A1 & 11 & $55 \%$ & 2 & $20 \%$ & 1 & $11.1 \%$ & 0 & $0 \%$ \\
A2 & 8 & $40 \%$ & 4 & $50 \%$ & 5 & $55.6 \%$ & 0 & $0 \%$ \\
B1 & 1 & $5 \%$ & 3 & $30 \%$ & 2 & $22.2 \%$ & 4 & $66.7 \%$ \\
B2 or above & 0 & $0 \%$ & 1 & $10 \%$ & 1 & $11.1 \%$ & 2 & $33.3 \%$ \\
\hline
\end{tabular}

a. Survey I statistics.

b. Survey II statistics.

$\underline{\text { Technical Access and Ease of Use (TEC) }}$

Table 13 shows that the students had adequate internet access at home and the capabilities to access it, which were criteria for this blended-learning course. The students were competent in using an internet-enabled mobile device, laptop, or desktop. 92.2\% of Survey I participants and 95\% of Survey II respondents had a laptop or desktop at home. 
At least $86.2 \%$ of the students had internet access at home with at least $50 \%$ of them saying their internet speed was extremely or moderately fast. $90 \%$ of the participants reported that the speed of the internet was average or above. The participants from Survey II showed a slight preference toward mobile devices. Surveys I and II indicated that at least $70 \%$ of the students had their mobile phones connected to the internet frequently. Only one participant in Survey II either did not have a mobile device or did not connect it to the internet. At least $91.3 \%$ of the participants from Survey II reported that using the internet, a computer, or a smartphone was extremely, moderately, or slightly easy. 
Table 13: Demographics (Technical Access and Ease of Use) TEC1-8

\begin{tabular}{|c|c|c|c|c|}
\hline & \multicolumn{2}{|c|}{ Survey I } & \multicolumn{2}{|c|}{ Survey II } \\
\hline & $\mathrm{N}$ & $\%$ & $\mathrm{~N}$ & $\%$ \\
\hline \multicolumn{5}{|l|}{ Mobile phone internet connection ${ }^{a}$} \\
\hline Always to Frequently (1-3) & 26 & $70.6 \%$ & 69 & $86.3 \%$ \\
\hline $50 \%$ to Occasionally (4-5) & 13 & $25.5 \%$ & 9 & $11.3 \%$ \\
\hline \multicolumn{5}{|l|}{ Computer at home ${ }^{b}$} \\
\hline Desktop & 26 & $51.0 \%$ & 38 & $47.5 \%$ \\
\hline Laptop & 37 & $72.5 \%$ & 69 & $86.3 \%$ \\
\hline Tablet & 12 & $23.5 \%$ & 11 & $13.8 \%$ \\
\hline No desktop or laptop & 4 & $7.8 \%$ & 4 & $5 \%$ \\
\hline \multicolumn{5}{|l|}{ Prefers browsing the internet using } \\
\hline Mobile device & 12 & $23.5 \%$ & 42 & $52.5 \%$ \\
\hline Desktop/laptop & 4 & $7.8 \%$ & 5 & $6.3 \%$ \\
\hline No preference & 35 & $68.6 \%$ & 33 & $41.3 \%$ \\
\hline \multicolumn{5}{|l|}{ Home internet connection type } \\
\hline Fiber optics or DSL & 23 & $45.1 \%$ & 22 & 27.50 \\
\hline 3G/4G modem & 11 & $21.6 \%$ & 26 & $32.5 \%$ \\
\hline Dial-up, other, or does not know & 15 & $29.4 \%$ & 27 & $33.75 \%$ \\
\hline No home internet & 2 & $3.9 \%$ & 11 & $13.8 \%$ \\
\hline \multicolumn{5}{|l|}{ Home internet connection speed ${ }^{\mathrm{c}}$} \\
\hline Extremely or moderately fast & 26 & $51.0 \%$ & 40 & $50 \%$ \\
\hline Slightly fast or average & 22 & $43.1 \%$ & 32 & $40 \%$ \\
\hline Slightly slow or slower & 3 & $5.9 \%$ & 8 & $10.0 \%$ \\
\hline Internet ease of use ${ }^{\mathrm{d}}$ & - & - & & \\
\hline Extremely to slightly easy & - & - & 78 & $97.5 \%$ \\
\hline Neither easy nor difficult to slightly difficult & - & - & 2 & $2.5 \%$ \\
\hline Computer or laptop ease of use ${ }^{d}$ & - & - & & \\
\hline Extremely to slightly easy & - & - & 73 & $91.3 \%$ \\
\hline Neither easy nor difficult to slightly difficult & - & - & 7 & $8.8 \%$ \\
\hline Smartphone ease of use ${ }^{d}$ & - & - & & \\
\hline Extremely to slightly easy & - & - & 78 & $97.5 \%$ \\
\hline Neither easy nor difficult to slightly difficult & - & - & 2 & $2.5 \%$ \\
\hline \multicolumn{5}{|c|}{$\begin{array}{l}\text { a. Scale of 1-7: Always, Very Frequently, Frequently, } 50 \% \text {, Occasionally, Rarely, Never. } \\
\text { b. Totals will be more than } 100 \% \text { because some participants have more than one type. } \\
\text { c. Scale of 1-7: Extremely fast, Moderately fast, Slightly fast, Average, Slightly slow, } \\
\text { Moderately slow, Extremely slow (or no internet). } \\
\text { d. Scale of 1-7: Extremely easy, Moderately easy, Slightly easy, Neither easy nor } \\
\text { difficult, Slightly difficult, Moderately difficult, Extremely difficult. }\end{array}$} \\
\hline
\end{tabular}




\section{$\underline{\text { Previous Elearning Experience (PEX) }}$}

This project collected the participants' previous elearning experience during Survey $\mathrm{I}(\mathrm{N}=51)$ but omitted the questions in the posttests after determining that it was uncommon for a student to have elearning experience. Even though $23 \%$ of the students in Table 14 indicated that their high school had an online learning portal, only $13.7 \%$ said their teacher used it.

The results in Table 15 show that three participants used an online learning portal at least occasionally at their high school. During a conversation, several of the students in the researcher's class indicated that they attended a Nazarbayev Intellectual School or a Turkish high school. It is likely that the few students who studied at a privileged school would have experienced using an online portal for learning.

Table 14: $\quad$ Previous Elearning Experience (PEX1-2)

\begin{tabular}{lcccccc}
\hline & \multicolumn{2}{c}{ Yes } & \multicolumn{2}{c}{ No } & \multicolumn{2}{c}{ Don't Know } \\
& $\mathrm{N}$ & $\%$ & $\mathrm{~N}$ & $\%$ & $\mathrm{~N}$ & $\%$ \\
\hline My high school had: & & & & & & \\
A student-use computer lab & 45 & $88.2 \%$ & 3 & $5.9 \%$ & 3 & $5.9 \%$ \\
An online learning portal & 12 & $23.5 \%$ & 38 & $74.5 \%$ & 1 & $2.0 \%$ \\
\hline
\end{tabular}

Note: Total number of respondents for this data is 51 . 
Table 15: $\quad$ Previous Elearning Experience (PEX3-7)

\begin{tabular}{|c|c|c|c|c|c|c|}
\hline & $\begin{array}{l}\text { Fre } \\
\text { Occ }\end{array}$ & $\begin{array}{l}\text { lery } \\
\text { iently to } \\
\text { ionally }\end{array}$ & & arely ${ }^{a}$ & & ever ${ }^{a}$ \\
\hline & $\mathrm{N}$ & $\%$ & $\mathrm{~N}$ & $\%$ & $\mathrm{~N}$ & $\%$ \\
\hline At my high school: & & & & & & \\
\hline I used an online learning portal. & 6 & $11.8 \%$ & 5 & $9.8 \%$ & 40 & $78.4 \%$ \\
\hline $\begin{array}{l}\text { I submitted assignments through } \\
\text { an online learning portal. }\end{array}$ & 2 & $3.9 \%$ & 4 & $7.8 \%$ & 45 & $88.2 \%$ \\
\hline $\begin{array}{l}\text { I accessed course material through } \\
\text { an online learning portal. }\end{array}$ & 3 & $5.9 \%$ & 5 & $9.8 \%$ & 43 & $84.3 \%$ \\
\hline $\begin{array}{l}\text { My teacher posted assignments to } \\
\text { an online learning portal. }\end{array}$ & 3 & $5.9 \%$ & 7 & $13.7 \%$ & 41 & $80.4 \%$ \\
\hline $\begin{array}{l}\text { My teacher communicated with us } \\
\text { through an online learning portal. }\end{array}$ & 3 & $5.9 \%$ & 5 & $9.8 \%$ & 43 & $84.3 \%$ \\
\hline
\end{tabular}

Note: Total number of respondents for this data is 51.

a. Scale of 1-7: Always, Very Frequently, Frequently, 50\%, Occasionally, Rarely, Never.

\section{Findings Overview}

$\underline{\text { Research Question } 1 \text { Results }}$

The results from the data do not conclusively prove or disprove research question

1, which sought to determine if PU had a stronger influence than PEOU on ATT in

Kazakhstan. The results from all three surveys as shown in Table 16 demonstrate that

Surveys I and III did not support this supposition, but the analysis of Survey II did.

Table 16: $\quad$ Effects of PU and PEOU on ATT

\begin{tabular}{ccccc}
\hline & \multicolumn{2}{c}{ Direct Effects on ATT } & \multicolumn{2}{c}{ Total Effects on ATT } \\
Survey & PU $\rightarrow$ ATT & PEOU $\rightarrow$ ATT & PU $\rightarrow$ ATT & PEOU $\rightarrow$ ATT \\
\hline I $^{\mathrm{a}}$ & 0.289 & 0.334 & 0.289 & 0.498 \\
II $^{\mathrm{b}}$ & 0.441 & 0.073 & 0.441 & 0.223 \\
III $^{\mathrm{c}}$ & 0.068 & 0.294 & 0.068 & 0.321 \\
\hline
\end{tabular}

a. Table 21

b. Table 23

c. Table 25 


\section{$\underline{\text { Research Question } 2 \text { Results }}$}

TAM is inconsistent in Kazakhstan when using the original TAM model. In short, the results from the original TAM model in Kazakhstan using the factors of PU, PEOU, ATT, and BI are different from the results in developed countries. Most notably, the ATT construct demonstrated inconsistencies, as displayed in Table 17. The parts of the model that compared consistently to results in developed countries were the impact of PEOU on PU and then the influence of PU on BI.

Table 17: $\quad$ Results in Kazakhstan (Original TAM)

\begin{tabular}{cccccc}
\hline PEOU $\rightarrow$ & PEOU $\rightarrow$ & PU & PU & ATT & \\
PU & ATT & $\rightarrow$ ATT & $\rightarrow$ BI & $\rightarrow$ BI & Dataset \\
\hline $0.566^{* *}$ & $0.334^{*}$ & 0.289 & $0.290^{*}$ & $0.345^{*}$ & Survey I \\
$0.341^{*}$ & 0.073 & $0.441^{*}$ & $0.246^{*}$ & $0.295^{*}$ & Survey II \\
$0.392^{*}$ & 0.294 & 0.068 & $0.385^{*}$ & 0.122 & Survey III \\
\hline
\end{tabular}

Significance Levels: $* p<0.05, * * p<0.01$.

Additionally, the results from the simplified TAM model in Kazakhstan using the factors of PU, PEOU, and BI in Kazakhstan are different from the significant factors in developed countries. Table 18 shows that the impact of PEOU on BI did not show significance in Kazakhstan, but it did in developed countries. This project expected these abnormal results using the simplified TAM model based on prior research and Kazakhstan's cultural dimensions, as shown in Table 35. Moreover, Table 18 shows that the simplified TAM version exhibits reliability in Kazakhstan, even if the results are different than in developed countries. 
Table 18: $\quad$ Results in Kazakhstan (Simplified TAM)

\begin{tabular}{cccc}
\hline $\mathrm{PEOU} \rightarrow \mathrm{PU}$ & $\mathrm{PEOU} \rightarrow \mathrm{BI}$ & $\mathrm{PU} \rightarrow \mathrm{BI}$ & Dataset \\
\hline $0.669 * *$ & 0.037 & $0.519 * *$ & Survey I \\
$0.389 * *$ & 0.046 & $0.361 * *$ & Survey II \\
$0.338^{*}$ & 0.254 & $0.410^{* *}$ & Survey III \\
\hline
\end{tabular}

Significance Levels: $* p<0.05, * * p<0.01$.

\section{PCA Analysis}

The principal component analysis of all three surveys confirms that the data are acceptable for component extraction and path analysis. First, the KMO values were all > 0.6 (Kaiser, 1974). Next, the communalities results were all above the threshold of 0.4 (Costello \& Osborne, 2005). Third, most of the values in the correlation matrices were greater than 0.3 (Williams et al., 2010), with the analysis of Survey II containing the lowest correlations. Finally, all extracted components except one met the lower threshold of accounting for at least $5 \%$ of the total variance (O’Rourke \& Hatcher, 2013). Tables 19 and 20 show the summary of the PCA analysis, which illustrates that PU consistently had the highest amount of variance in both the original and simplified TAM models.

\section{Table 19: $\quad$ PCA Overview (Original TAM}

\begin{tabular}{ccccccccc}
\hline Survey & \multicolumn{2}{c}{ Component $1^{\mathrm{a}}$} & \multicolumn{2}{c}{ Component $2^{\mathrm{a}}$} & \multicolumn{2}{c}{ Component $3^{\mathrm{a}}$} & \multicolumn{2}{c}{ Component $4^{\mathrm{a}}$} \\
I & PU & $58.88 \%$ & BI & $9.67 \%$ & PEOU & $5.26 \%$ & ATT & $4.98 \%$ \\
II & PU & $47.58 \%$ & PEOU & $16.28 \%$ & BI & $11.30 \%$ & ATT & $8.23 \%$ \\
III & PU & $52.38 \%$ & ATT & $11.33 \%$ & BI & $8.98 \%$ & PEOU & $7.06 \%$ \\
\hline
\end{tabular}

a. TAM factor and percent of total variance. 
Table 20: $\quad$ PCA Overview (Simplified TAM)

\begin{tabular}{ccrcrcc}
\hline Survey & \multicolumn{2}{c}{ Component $1^{\mathrm{a}}$} & \multicolumn{2}{c}{ Component $2^{\mathrm{a}}$} & \multicolumn{2}{c}{ Component $3^{\mathrm{a}}$} \\
I & PU & $58.04 \%$ & BI & $10.53 \%$ & PEOU & $5.64 \%$ \\
II & PU & $44.08 \%$ & PEOU & $18.54 \%$ & BI & $13.43 \%$ \\
III & PU & $52.17 \%$ & BI & $12.42 \%$ & PEOU & $8.85 \%$ \\
\hline
\end{tabular}

a. TAM factor and percent of total variance.

\section{Survey I PCA Results}

\section{Analysis using the Original TAM Model: Survey I}

The preliminary analysis of Survey I shows that the data meet most of the requirements for PCA and path analysis (See Appendix B, Tables B1-B7). The correlation matrix shows all values except two BI5 values in Table B1 met the threshold of $>0.3$ (Williams et al., 2010). The anti-image correlation matrix indicates that the data might have factorability problems because most values are not $>0.5$ (Kaiser $\&$ Rice, 1974). The KMO and Bartlett's Test value is .888, which is larger than the threshold of 0.6 (Kaiser, 1974). The communality estimates are all greater than the limit of 0.4 (Costello \& Osborne, 2005).

The cross-validation procedure extracted the TAM components of PU, PEOU, ATT, and BI from the survey data with eigenvalues $>0.7$, which account for $78.79 \%$ of the variance (See Appendix B, Tables B5-B6). The top three components meet O'Rourke and Hatcher's (2013) threshold for retaining the factors that account for at least $5 \%$ of the total variance, with the last factor accounting for $4.98 \%$. The component correlation matrix supports using the oblique rotation with all correlations $>0.3$ (Table B7). The extracted TAM components ordered by variance explained are PU (58.88\%), BI (9.67\%), PEOU (5.26\%), and ATT (4.98\%). 


\section{Analysis using the Simplified TAM Model: Survey I}

The preliminary analysis shows that the data from Survey I meet most of the requirements for PCA and a path analysis after removing the ATT construct (See Appendix B, Tables B8-B13). The correlation matrix shows all values except three from BI5 in Table B8 are > 0.3. The KMO and Bartlett's Test value is .886. The communality estimates are all $>0.4$.

The cross-validation procedure extracted the TAM components of PU, PEOU, and BI from the survey data with eigenvalues $>0.7$, which account for $74.21 \%$ of the variance (See Appendix B, Tables B11-B12). Each of the three components accounts at least $5 \%$ of the total variance. The component correlations matrix supports using the oblique rotation with all correlations $>0.3$ (Table B13). The extracted TAM components ordered by variance explained are PU (58.04\%), BI (10.53\%), and PEOU (5.64\%).

\section{$\underline{\text { Survey II PCA Results }}$}

The PCA results identified a problem with Survey II. Factors PU1-PU2 correlate with each other as do PU3-PU4. However, PU1 or PU2 do not correlate with PU3 or PU4. A discovery was made that the survey includes questions for PU1-PU2 in the same block and then question PU3-PU4 in a different block. The respondents answered the questions in the same block similarly, but their answers were not consistent between the two blocks. PU3 and PU4 produced more stable results than using PU1 and PU2. Therefore, variables PU1 and PU2 were removed.

\section{Analysis using the Original TAM Model: Survey II}

The preliminary analysis of Survey II shows that the data meet most of the requirements for PCA and path analysis (See Appendix C, Tables C1-C7). The 
correlation matrix shows that the variables B1, B2, and PEOU5 have low correlation values. The anti-image correlation matrix indicates that the data might have factorability problems because most values are smaller than the threshold of 0.5 . The KMO and Bartlett's Test value of .780 is $>0.6$. The communality estimates are all $>0.4$.

The cross-validation procedure extracted the TAM components of PU, PEOU, ATT, and BI from the survey data with eigenvalues $>0.6$, which account for $83.39 \%$ of the variance (See Appendix C, Tables C5-C7). All four components account for at least $5 \%$ of the total variance. The component correlation matrix shows that factor two, PEOU, has low correlation values (Table C7). The extracted TAM components ordered by variance explained are PU (47.58\%), PEOU (16.28\%), BI (11.30\%), and ATT (8.234\%).

\section{Analysis using the Simplified TAM Model: Survey II}

The preliminary analysis shows that the data from Survey II meet most of the requirements for PCA and a path analysis after removing the ATT construct (See Appendix C, Tables C8-C13). The correlation matrix shows that most of the variables have correlation values lower than the threshold of 0.3 signifying the data could be problematic. The KMO and Bartlett's Test value is .711. The communality estimates are all $>0.4$.

The cross-validation procedure extracted the TAM components of PU, PEOU, and BI from the survey data with eigenvalues $>1$, which account for $76.04 \%$ of the variance (See Appendix C, Tables C11-C12). Each of the extracted components accounts for at least $10 \%$ of the total variance. The component correlation matrix shows one low correlation value (Table C13). The extracted TAM components ordered by variance explained are PU (44.08\%), PEOU (18.54\%), and BI (13.43\%). 


\section{$\underline{\text { Survey III PCA Results }}$}

\section{Analysis using the Original TAM Model: Survey III}

The preliminary analysis of Survey III shows that the data meet most of the requirements for PCA and path analysis (See Appendix D, Tables D1-D7). The correlation matrix shows that PEOU2 has low correlations values with the PU and BI components. The anti-image correlation matrix indicates that the data might have factorability problems because most values are not $>0.5$. The KMO and Bartlett's Test value is .822 . The communality estimates are all $>0.4$.

The cross-validation procedure extracted the TAM components of PU, PEOU, ATT, and BI from the survey data with eigenvalues $>0.7$, which account for $79.74 \%$ of the variance (See appendix D, Tables D5-D6). Each retained factor accounts for at least $5 \%$ of the total variance. The component correlations matrix shows potential problems because components PU, ATT, and BI have low correlation values (Table D7). The extracted TAM factors ordered by variance explained are PU (52.38\%), ATT (11.33\%), BI (8.98\%), and PEOU (7.06\%).

\section{Analysis using the Simplified TAM Model: Survey III}

The preliminary analysis shows that the data from Survey III meet most of the requirements for PCA and a path analysis after removing the ATT construct (See appendix D, Tables D8-D13). The correlation matrix shows problems with variable PEOU2. The KMO and Bartlett's Test value is .800. The communality estimates are all > 0.4 .

The cross-validation procedure extracted the TAM components of PU, PEOU, and BI from the survey data with eigenvalues $>.9$, which account for $73.45 \%$ of the 
variance (See appendix D, Tables D11-D12). Each of the three components accounts for at least $8 \%$ of the total variance. The component correlation matrix shows that factors are larger than 0.3 (Table D13). The extracted TAM components ordered by variance explained are PU (52.17\%), BI (12.42\%), and PEOU (8.85\%).

\section{Path Analysis (PA)}

The path analysis procedure on the original TAM using the PCA components from Surveys I, II, and III showed inconsistencies. First, the correlations among factors were all significant using the results from Surveys I and II, but Survey III contained two non-significant correlations. Next, only paths PEOU $\rightarrow$ PU and PU $\rightarrow$ BI were significant in all three analyses. Additionally, the variable that had the greatest impact on BI was not consistent across all surveys. However, the total effect of PU on BI consistently had the highest influence among all three surveys.

Contrary to the analyses on the original TAM, the results of the simplified TAM model showed less deviation among all three data sets. The paths of PEOU $\rightarrow$ PU and $\mathrm{PU} \rightarrow \mathrm{BI}$ were always significant while $\mathrm{PEOU} \rightarrow \mathrm{BI}$ was never significant. The analysis of Survey II resulted in a non-significant correlation between PEOU and BI. The most substantial inconsistency is that the total effects from the Survey III analysis did not align with the results from Survey I and II.

\section{Survey I PA Results}

Table 21 and Figure 6 show the path analysis results using the original TAM model. The only non-significant path is PU $\rightarrow$ ATT. The impact of PEOU on PU has the highest total effect, but PU has the most overall influence on BI. 
Table 22 and Figure 7 shows the path analysis results using the simplified TAM model. All correlations are significant, but the only non-significant path is PEOU $\rightarrow$ BI.

Table 21: $\quad$ Survey I Path Analysis Results (Original TAM)

\begin{tabular}{|c|c|c|c|c|c|}
\hline \multicolumn{2}{|c|}{ Variables } & \multirow[b]{2}{*}{ Correlation } & \multicolumn{3}{|c|}{ Direct Effect } \\
\hline$X$ (ind) & Y (dep) & & $\mathrm{R}$ & (Beta) & Total Effect \\
\hline PEOU & PU & $0.566 * *$ & 0.566 & $0.566^{* *}$ & 0.566 \\
\hline PEOU & ATT & $0.498 * *$ & \multirow{2}{*}{0.552} & $0.334^{*}$ & 0.498 \\
\hline PU & ATT & $0.478 * *$ & & 0.289 & 0.289 \\
\hline PU & $\mathrm{BI}$ & $0.455 * *$ & \multirow{2}{*}{0.546} & $0.290 *$ & 0.390 \\
\hline ATT & $\mathrm{BI}$ & $0.484 * *$ & & $0.345^{*}$ & 0.345 \\
\hline PEOU & $\mathrm{BI}$ & - & - & - & 0.336 \\
\hline
\end{tabular}

Significance Levels: $* p<0.05, * * p<0.01$.

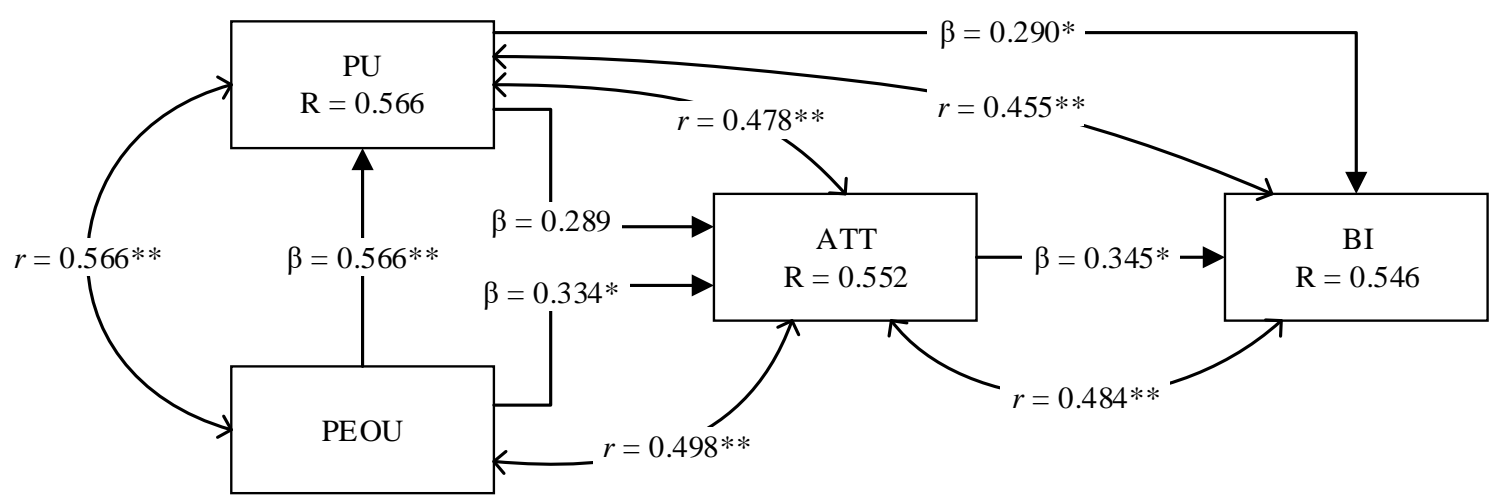

Figure 6: $\quad$ Survey I Results (Original TAM)

Table 22: $\quad$ Survey I Path Analysis Results (Simplified TAM)

\begin{tabular}{cccccc}
\hline \multicolumn{2}{c}{ Variables } & & \multicolumn{3}{c}{ Direct Effect } \\
X (ind) & Y (dep) & Correlation & R & (Beta) & Total Effect \\
\hline PEOU & PU & $0.669 * *$ & 0.669 & $0.669 * *$ & 0.669 \\
PU & BI & $0.544 * *$ & & $0.519 * *$ & 0.519 \\
PEOU & BI & $0.385^{* *}$ & 0.545 & 0.037 & 0.384 \\
\hline
\end{tabular}

Significance Levels: $* p<0.05, * * p<0.01$. 


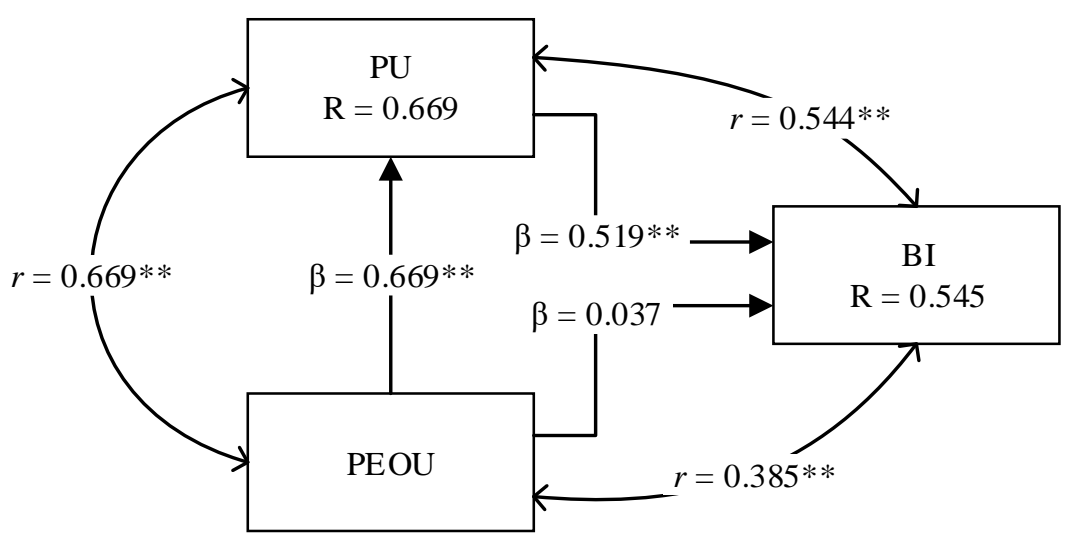

Figure 7: $\quad$ Survey Results (Simplified TAM)

\section{Analysis of the Original TAM Model: Survey I}

The first regression analysis was conducted on the impact of PEOU on PU (See appendix E Tables, E1-E3), which shows that a significant correlation exists between PEOU and PU. Furthermore, the path coefficient from PEOU to PU is significant (Table E3). The model summary table shows that PU accounts for $32 \%$ of the variance.

The second regression analysis was conducted on the impact of PEOU and PU on ATT (See appendix E, Tables E4-E6), which shows that significant correlations exist between PU, PEOU, and ATT. Additionally, the path PEOU $\rightarrow$ ATT is significant, but path PU $\rightarrow$ ATT is not (Table E6). The model summary table shows that ATT accounts for $30.5 \%$ of the variance with predictors PEOU and PU.

The third regression analysis was conducted on the impact of PU and ATT on BI (See appendix E Tables, E7-E9), which shows that significant correlations exist between PU, ATT, and BI. Furthermore, the paths $\mathrm{PU} \rightarrow \mathrm{BI}$ and ATT $\rightarrow$ BI are significant (Table E9). The model summary table shows that BI accounts for $29.8 \%$ of the variance with predictors PU and ATT. 


\section{Analysis of the Simplified TAM Model: Survey I}

The correlations analysis of the simplified TAM model using Survey I shows that significant correlations exist between PU, PEOU, and BI (See appendix E Table E10). The first regression analysis was conducted on the impact of PEOU on PU, which shows that the path coefficient from PEOU to PU is significant (Table E12). The model summary table shows that PU accounts for $44.8 \%$ of the variance. The second regression analysis was conducted on the impact of PEOU on BI and the impact of PU on BI. The results show that path $\mathrm{PEOU} \rightarrow \mathrm{BI}$ is not significant, but $\mathrm{PU} \rightarrow \mathrm{BI}$ is significant (Table E14). The model summary table shows that BI accounts for $29.7 \%$ of the variance with predictors PEOU and PU (Table E13).

\section{$\underline{\text { Survey II PA Results }}$}

Table 23 and Figure 8 show the path analysis results using the original TAM model. The only non-significant path is PEOU $\rightarrow$ ATT, which is a deviation from the results of Survey I. The impact of PU on ATT has the highest total effect, with PU having the most overall influence on BI.

Table 24 and Figure 9 show the path analysis results using the simplified TAM model. Unlike the results of Survey I, the correlation between PEOU and BI is not significant. Conversely, both surveys show that the path PEOU $\rightarrow$ BI is not significant. 
Table 23: $\quad$ Survey II Path Analysis Results (Original TAM)

\begin{tabular}{|c|c|c|c|c|c|}
\hline \multicolumn{2}{|c|}{ Variables } & \multirow[b]{2}{*}{ Correlation } & \multicolumn{3}{|c|}{ Direct Effect } \\
\hline$X$ (ind) & $\mathrm{Y}(\mathrm{dep})$ & & $\mathrm{R}$ & (Beta) & Total Effect \\
\hline PEOU & PU & $0.341^{* *}$ & 0.341 & $0.341 * *$ & 0.341 \\
\hline PEOU & ATT & $0.223 *$ & \multirow{2}{*}{0.471} & 0.073 & 0.223 \\
\hline $\mathrm{PU}$ & ATT & $0.466^{* *}$ & & $0.441 * *$ & 0.441 \\
\hline $\mathrm{PU}$ & BI & $0.383 * *$ & \multirow{2}{*}{0.464} & $0.246^{*}$ & 0.376 \\
\hline ATT & BI & $0.409^{* *}$ & & $0.295^{*}$ & 0.295 \\
\hline PEOU & BI & - & - & - & 0.150 \\
\hline
\end{tabular}

Significance Levels: $* p<0.05, * * p<0.01$.

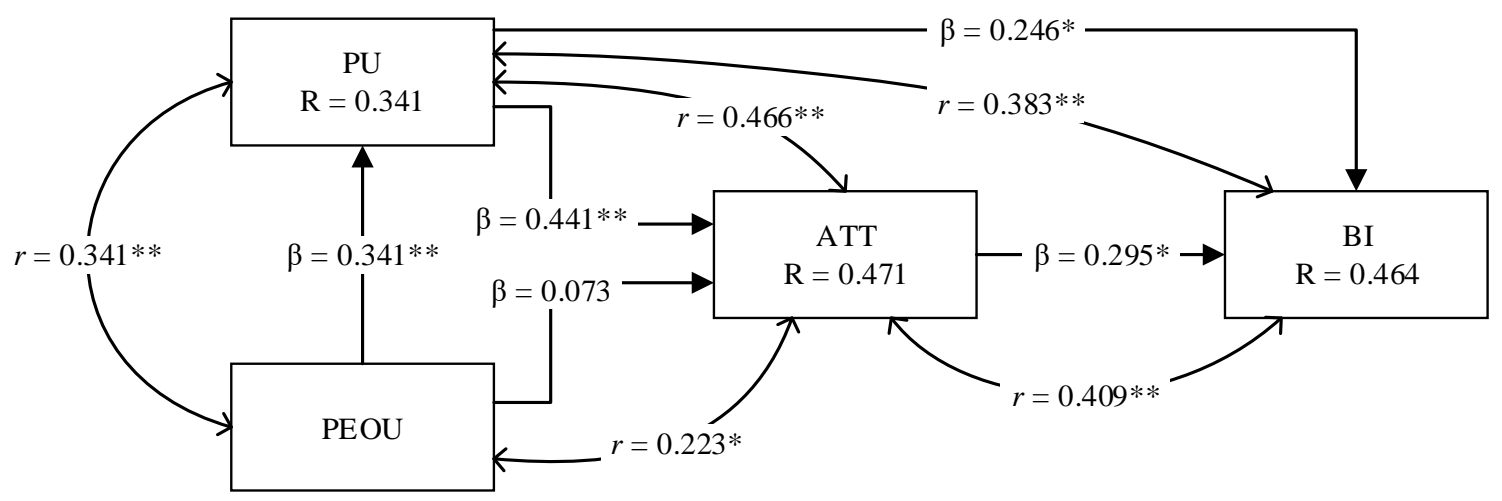

Figure 8: $\quad$ Survey II Results (Original TAM)

Table 24: $\quad$ Survey II Path Analysis Results (Simplified TAM)

\begin{tabular}{|c|c|c|c|c|c|}
\hline \multicolumn{2}{|c|}{ Variables } & \multirow[b]{2}{*}{ Correlation } & \multicolumn{3}{|c|}{ Direct Effect } \\
\hline$X$ (ind) & $Y(d e p)$ & & $\mathrm{R}$ & (Beta) & Total Effect \\
\hline PEOU & PU & $0.389 * *$ & 0.389 & $0.389 * *$ & 0.389 \\
\hline PU & BI & $0.379 * *$ & \multirow{2}{*}{0.381} & $0.361 * *$ & 0.361 \\
\hline PEOU & BI & 0.187 & & 0.046 & 0.186 \\
\hline
\end{tabular}

Significance Levels: $* p<0.05, * * p<0.01$. 


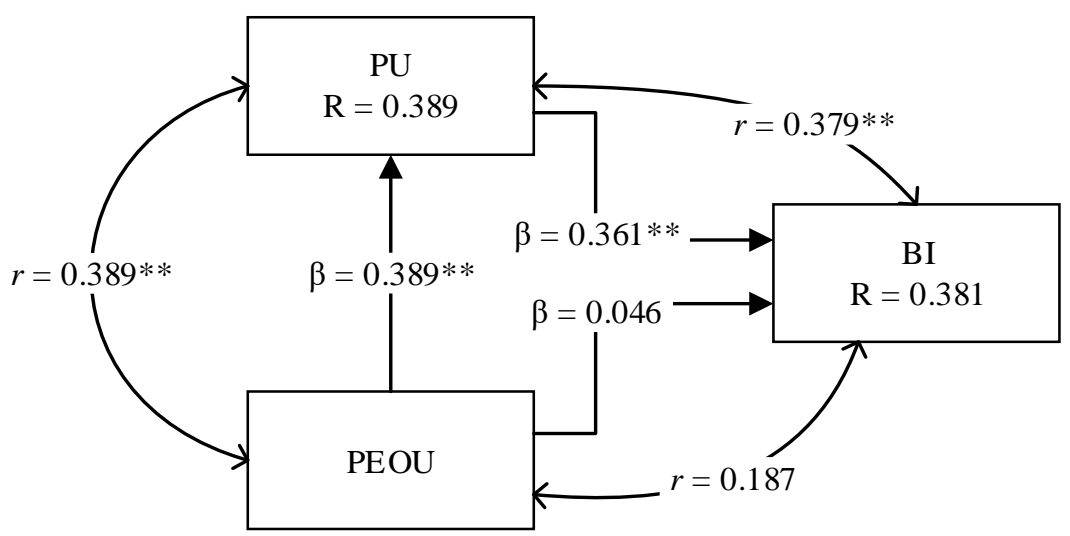

Figure 9: $\quad$ Survey II Results (Simplified TAM)

\section{Analysis of the Original TAM Model: Survey II}

The first regression analysis was conducted on the impact of PEOU on PU (See appendix F, Tables F1-F3), which shows that a significant correlation exists between PEOU and PU. Furthermore, the path coefficient from PEOU to PU is significant (Table F3). The model summary table shows that PU accounts for $11.6 \%$ of the variance.

The second regression analysis was conducted on the impact of PEOU and PU on ATT (See appendix F, Tables F4-F6), which shows that significant correlations exist between PU, PEOU, and ATT. Path PU $\rightarrow$ ATT is significant, but PEOU $\rightarrow$ ATT is not significant (Table F6). The model summary table shows that ATT accounts for $22.1 \%$ of the variance with predictors PEOU and PU.

The third regression analysis was conducted on the impact of PU and ATT on BI (Appendix F, Tables F7-F9), which shows that significant correlations exist between PU, ATT, and BI. Furthermore, the paths PU $\rightarrow$ BI and ATT $\rightarrow$ BI are significant (Table F9). The model summary table reveals that BI accounts for $21.5 \%$ of the variance with predictors PU and ATT. 


\section{Analysis of the Simplified TAM Model: Survey II}

The correlations analysis on the simplified TAM model using Survey II showed that significant correlations exist between path PU and PEOU and path PU and BI but not between PEOU and BI (See appendix F Table F10). The first regression analysis was conducted on the impact of PEOU on PU, which shows that the path coefficient from PEOU to PU is significant (Table F12). The model summary table shows that PU accounts for $15.2 \%$ of the variance. The second regression analysis was conducted on the impact of PEOU on BI and the impact of PU on BI. The results show that path PEOU $\rightarrow$ $\mathrm{BI}$ is not significant, but $\mathrm{PU} \rightarrow \mathrm{BI}$ is significant (Table F14). The model summary table shows that BI accounts for $14.5 \%$ of the variance with predictors PEOU and PU (Table F13).

\section{Survey III PA Results}

Table 25 and Figure 10 show the path analysis results using the original TAM model. The TAM analysis deviated from the previous two models since it had two nonsignificant correlations and three non-significant paths. The impact of PU on BI has the highest total effect.

Table 26 and Figure 11 show the path analysis results using the simplified TAM model. Comparable to the results of Survey I, all correlations are significant, and the only non-significant path is PEOU $\rightarrow$ BI. Contrary to the analyses on Surveys I and II, the path with the most influence is $\mathrm{PU} \rightarrow \mathrm{BI}$ instead of PEOU $\rightarrow$ PU. 
Table 25: $\quad$ Survey III Path Analysis Results (Original TAM)

\begin{tabular}{|c|c|c|c|c|c|}
\hline \multicolumn{2}{|c|}{ Variables } & \multirow[b]{2}{*}{ Correlation } & \multicolumn{3}{|c|}{ Direct Effect } \\
\hline$X$ (ind) & Y (dep) & & $\mathrm{R}$ & (Beta) & Total Effect \\
\hline PEOU & PU & $0.392 * *$ & 0.392 & $0.392 * *$ & 0.392 \\
\hline PEOU & ATT & $0.320^{*}$ & \multirow{2}{*}{0.326} & 0.294 & 0.321 \\
\hline PU & ATT & 0.183 & & 0.068 & 0.068 \\
\hline PU & BI & $0.407 * *$ & \multirow{2}{*}{0.424} & $0.385^{*}$ & 0.393 \\
\hline ATT & BI & 0.192 & & 0.122 & 0.122 \\
\hline PEOU & BI & - & - & - & 0.190 \\
\hline
\end{tabular}

Significance Levels: $* p<0.05,{ }^{* *} p<0.01$.

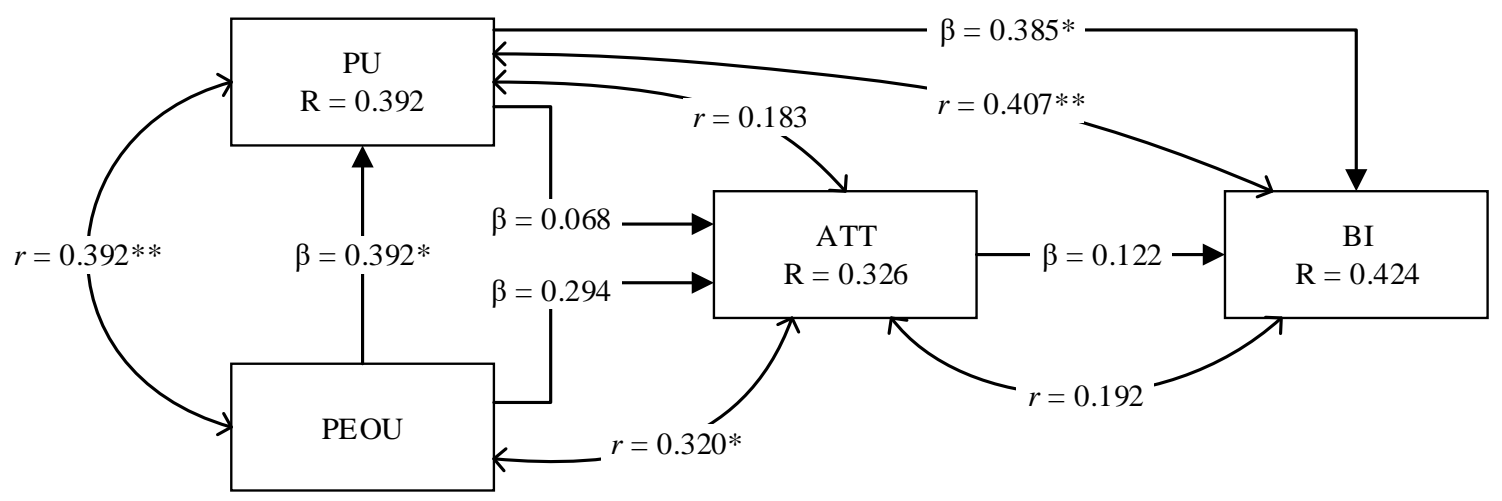

Figure 10: Survey III Results (Original TAM)

Table 26: $\quad$ Survey III Path Analysis Results (Simplified TAM)

\begin{tabular}{|c|c|c|c|c|c|}
\hline \multicolumn{2}{|c|}{ Variables } & \multirow[b]{2}{*}{ Correlation } & \multicolumn{3}{|c|}{ Direct Effect } \\
\hline$X$ (ind) & $\mathrm{Y}(\mathrm{dep})$ & & $\mathrm{R}$ & (Beta) & Total Effect \\
\hline PEOU & PU & $0.338^{*}$ & 0.338 & $0.338^{*}$ & 0.338 \\
\hline PU & BI & $0.495 * *$ & \multirow{2}{*}{0.550} & $0.410^{* *}$ & 0.410 \\
\hline PEOU & BI & $0.392 * *$ & & 0.254 & 0.393 \\
\hline
\end{tabular}

Significance Levels: $* p<0.05, * * p<0.01$. 


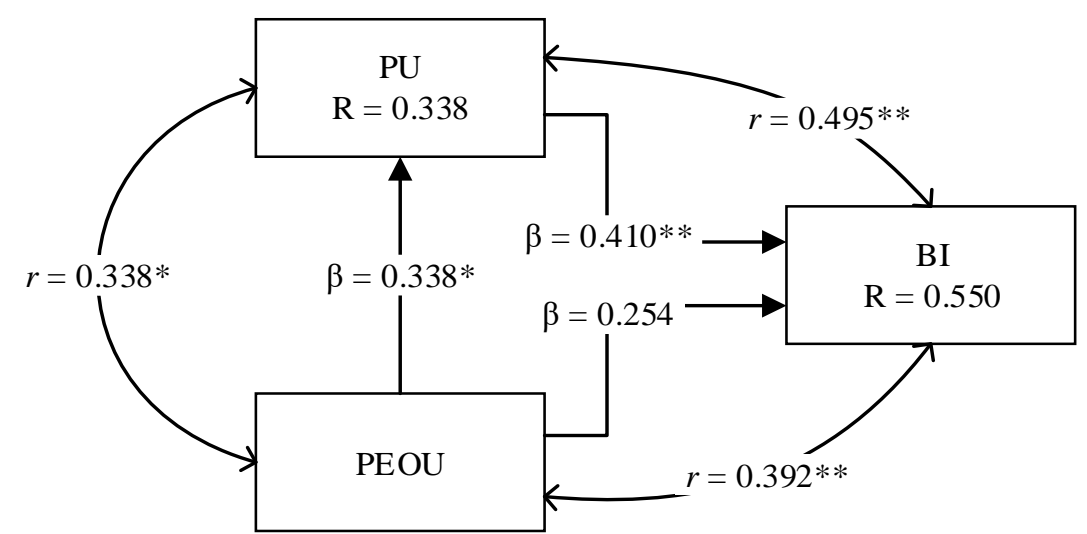

\section{Figure 11: $\quad$ Survey III Results (Simplified TAM)}

\section{Analysis of the Original TAM Model: Survey III}

The first regression analysis was conducted on the impact of PEOU on PU (See appendix G, Tables G1-G3), which shows that a significant correlation exists between PEOU and PU. Furthermore, the path coefficient from PEOU to PU is significant (Table G3). The model summary table shows that PU accounts for $15.4 \%$ of the variance.

The second regression analysis was conducted on the impact of PEOU and PU on ATT (See appendix G, Tables G4-G6), which shows that significant correlations exist between PEOU and PU and also between PEOU and ATT, but not between PU and ATT. The regression analysis revealed that paths $\mathrm{PU} \rightarrow \mathrm{ATT}$ and PEOU $\rightarrow$ ATT were not significant (Table G6). The model summary table shows that ATT accounts for $10.6 \%$ of the variance with predictors PEOU and PU.

The third regression analysis was conducted on the impact of PU and ATT on BI (See appendix G, Tables G7-G9), which shows that a significant correlation exists between PU and BI, but not between PU and ATT nor ATT and BI. Furthermore, the path PU $\rightarrow$ BI is significant, but ATT $\rightarrow$ BI is not (Table G9). The model summary table reveals that BI accounts for $18.0 \%$ of the variance with predictors PU and ATT. 


\section{Analysis of the Simplified TAM Model: Survey III}

The correlations analysis of the simplified TAM model using Survey III shows that a significant correlation exists between PU, PEOU, and BI but not between PEOU and BI (See appendix G Table G10). The first regression analysis was conducted on the impact of PEOU on PU, which shows that the path coefficient from PEOU to PU is significant (Table G12). The model summary table shows that PU accounts for $11.4 \%$ of the variance. The second regression analysis was conducted on the impact of PEOU on $\mathrm{BI}$ and the impact of PU on BI. The results show that path PEOU $\rightarrow \mathrm{BI}$ is not significant, but PU $\rightarrow$ BI is significant (Table G14). The model summary table shows that BI accounts for $30.3 \%$ of the variance with predictors PEOU and PU (Table G13).

\section{Comparative Analysis}

The comparative analysis uses the TAM data from Kazakhstan and other studies to determine if the results are comparable. This project bases the first comparison on King and He's (2006) analysis that revealed that PU should be the stronger influencer of BI. This comparative test is a simple way to determine that one aspect of TAM in Kazakhstan is the same as in other countries. The second analysis examines which paths are significant to evaluate how similar Kazakhstan is to other developed countries. Together, these comparisons describe the similarities and differences of the TAM models.

\section{$\underline{\text { Research Question } 1 \text { Analysis }}$}

The direct and indirect effects from PU and PEOU on ATT were evaluated from each dataset to answer the first research question. Table 16 shows that PEOU has the greatest impact on ATT for Survey I and III when evaluating both the direct and indict 
effects. Contrarily, Survey II reveals that PU has more influence on ATT than does PEOU.

Research Question 2 Analysis

The comparative analysis answered the second research question by evaluating existing TAM studies in developed countries using two criteria. First, the comparative analysis evaluated the number of significant paths in each TAM analysis. Then, it assessed which specific TAM paths were not significant. The expected result was that all paths on a successful TAM implementation in developed countries should be significant. However, Table 6 demonstrates that this expectation is not realistic. Instead, TAM studies commonly have one or more paths that are not significant. King and He (2006) confirm this supposition by concluding that 30 out of 67 studies that they analyzed resulted path PEOU $\rightarrow$ BI being non-significant on the simplified TAM model.

The studies used for the comparison came from Tables 6 and 8 . Table 6 lists 12 relevant TAM studies in educational contexts that used the original TAM model. Table 8 combines ten studies in educational and professional contexts that used the simplified TAM model. Only two of these ten studies from developed countries are in an educational setting. The overall conclusion is that the significant factors used both the original and simplified TAM models in Kazakhstan are different from the significant factors in developed countries.

\section{$\underline{\text { Results in Developed Countries }}$}

Table 27 shows the paths that are significant the most frequently among the 12 studies that used the original TAM in developed countries, which are PEOU $\rightarrow$ PU $(\mathrm{N}=12), \mathrm{PU} \rightarrow \operatorname{ATT}(\mathrm{N}=11)$, and PEOU $\rightarrow$ ATT $(\mathrm{N}=10)$. Conversely, ATT $\rightarrow$ BI $(\mathrm{N}=8)$ 
was statistically significant less frequently, with $\mathrm{PU} \rightarrow \mathrm{BI}(\mathrm{N}=5)$ being non-significant more often.

Table 27: $\quad$ Number of Significant Paths (Original TAM)

\begin{tabular}{lc}
\hline \multicolumn{1}{c}{ Path } & Significant Count $^{\mathrm{a}}$ \\
\hline $\mathrm{PEOU} \rightarrow$ PU & 12 \\
$\mathrm{PU} \rightarrow$ ATT & 11 \\
$\mathrm{PEOU} \rightarrow$ ATT & 10 \\
$\mathrm{ATT} \rightarrow$ BI & 8 \\
$\mathrm{PU} \rightarrow$ BI & 5 \\
\hline
\end{tabular}

Note: $\mathrm{N}=12$ from TAM studies in developed countries in Table 6 .

a. The number of significant paths from studies.

Table 28 examines the 12 studies that used the original TAM model in developed countries to determine how often one or more paths were not significant, which revealed that ten of the studies had at least one non-significant path. Seven of these ten studies had only one non-significant path, two studies had two paths that were not significant, and one study had three non-significant paths. Path PU $\rightarrow$ BI was not significant in seven studies, ATT $\rightarrow$ BI in four studies, PEOU $\rightarrow$ ATT in two studies, and PU $\rightarrow$ ATT in one study. Two studies shared the same non-significant path-pair, which is PU $\rightarrow$ BI and $\mathrm{ATT} \rightarrow \mathrm{BI}$. 
Table 28: Studies with non-Significant Paths (Original TAM)

\begin{tabular}{|c|c|}
\hline Studies that contain non-significant paths & Count \\
\hline One non-significant path ${ }^{\mathrm{a}}$ & 7 studies \\
\hline $\mathrm{PU} \rightarrow \mathrm{BI}$ & 4 \\
\hline $\mathrm{PEOU} \rightarrow \mathrm{ATT}$ & 2 \\
\hline $\mathrm{ATT} \rightarrow \mathrm{BI}$ & 1 \\
\hline Two non-significant paths ${ }^{b}$ & 2 studies \\
\hline $\mathrm{PU} \rightarrow \mathrm{BI} ; \mathrm{ATT} \rightarrow \mathrm{BI}^{\mathrm{e}}$ & 2 \\
\hline Three non-significant paths ${ }^{c}$ & 1 study \\
\hline $\mathrm{PU} \rightarrow \mathrm{BI} ; \mathrm{ATT} \rightarrow \mathrm{BI} ; \mathrm{PU} \rightarrow \mathrm{ATT}$ & 1 \\
\hline Four non-significant paths & 0 studies $^{\mathrm{d}}$ \\
\hline
\end{tabular}

Note: $\mathrm{N}=12$ from TAM studies in developed countries in Table 6 .

a. All paths were significant except 1 .

b. All paths were significant except 2 .

c. All paths were significant except 3 .

d. No study had four non-significant paths.

e. The same paths were not significant in different studies.

Table 29 shows the paths that are significant the most frequently among the ten studies using the simplified version of TAM in developed countries, which were PU $\rightarrow$ $\mathrm{BI}(\mathrm{N}=9), \mathrm{PEOU} \rightarrow \mathrm{BI}(\mathrm{N}=9)$, and $\mathrm{PEOU} \rightarrow \mathrm{PU}(\mathrm{N}=7)$.

Table 29: Number of Significant and Influential Paths (Simplified TAM)

\begin{tabular}{lc}
\hline \multicolumn{1}{c}{ Path } & Significant Count $^{\mathrm{a}}$ \\
\hline $\mathrm{PU} \rightarrow \mathrm{BI}$ & 9 \\
$\mathrm{PEOU} \rightarrow \mathrm{BI}$ & 9 \\
$\mathrm{PEOU} \rightarrow \mathrm{PU}$ & 7 \\
\hline
\end{tabular}

Note: $\mathrm{N}=10$ from TAM studies in developed countries in Table 8 .

Table 30 examines the ten studies that used the simplified TAM in developed countries to determine how often one or more paths were not significant, which revealed that only four out of ten studies have at least one non-significant path. Three of these 
studies had only one non-significant path, which was PEOU $\rightarrow$ PU. The other study had two paths that were not significant, which were $\mathrm{PU} \rightarrow \mathrm{BI}$ and $\mathrm{PEOU} \rightarrow \mathrm{BI}$.

Table 30: $\quad$ Studies with non-Significant Paths (Simplified TAM)

\begin{tabular}{lc}
\hline \multicolumn{1}{c}{ Studies that contain non-significant paths } & Count \\
\hline One non-significant path ${ }^{\mathrm{a}}$ & 3 studies \\
PEOU $\rightarrow$ PU & 3 \\
& \\
\hline Two non-significant paths ${ }^{\mathrm{b}}$ & 1 study \\
$\mathrm{PU} \rightarrow \mathrm{BI}$; PEOU $\rightarrow \mathrm{BI}^{\mathrm{e}}$ & 1 \\
& \\
\hline Three non-significant paths $^{\mathrm{c}}$ & 0 studies $^{\mathrm{d}}$ \\
\hline
\end{tabular}

Note: $\mathrm{N}=10$ from TAM studies in developed countries in Table 8 .

a. All paths were significant except for one.

b. All paths were significant except for two.

c. All paths were significant except for three.

d. No study had three non-significant paths.

e. The same paths were not significant in different studies.

\section{Comparison to Kazakhstan using the Original TAM}

The first comparison looked at the number of significant paths for each TAM analysis that used participants from Kazakhstan. Table 31 shows that each path analysis for this project had paths that were not significant. Having non-significant paths does not indicate a problem because Table 28 shows that ten of the twelve studies had one or more paths that were not significant. However, it was uncommon for studies to have two or more paths that were not significant. Table 31 shows that the results for Surveys I and II are comparable to studies in developed countries, but Survey III shows a deviation. This first comparison was inconclusive.

The next assessment examined which paths were not significant. The three path analyses in Kazakhstan revealed that PEOU $\rightarrow$ ATT and PU $\rightarrow$ ATT were not significant 
two times and ATT $\rightarrow$ BI was not significant once. According to Tables 27 and 28, paths $\mathrm{PEOU} \rightarrow \mathrm{ATT}$ and PU $\rightarrow$ ATT in developed countries were significant most of the time. Only one other study listed PU $\rightarrow$ ATT as not being statistically significant, and two studies reported that PEOU $\rightarrow$ ATT was not significant. This comparison indicated that the TAM factors in Kazakhstan could differ from those in developed countries.

The conclusion of the subjective comparison using the original version of TAM is that the impact of PEOU on PU and PU on BI is consistent with studies in developed countries. All other TAM factors differ by varying degrees.

Table 31: Comparison of Significant Values (Original TAM)

\begin{tabular}{lllll}
\hline \multicolumn{1}{c}{ Path } & \multicolumn{1}{c}{$\begin{array}{c}\text { Is Significance } \\
\text { Expected? }{ }^{\text {a }}\end{array}$} & \multicolumn{1}{c}{$\begin{array}{c}\text { Survey I } \\
\text { Results }\end{array}$} & \multicolumn{1}{c}{$\begin{array}{r}\text { Survey II } \\
\text { Results }\end{array}$} & \multicolumn{1}{c}{$\begin{array}{c}\text { Survey III } \\
\text { Results }\end{array}$} \\
\hline PEOU $\rightarrow$ PU & Yes & Same & Same & Same \\
PEOU $\rightarrow$ ATT & Yes & Same & Different & Different \\
PU $\rightarrow$ ATT & Yes & Different & Same & Different \\
PU $\rightarrow$ BI & Inconclusive & Same & & Same \\
ATT $\rightarrow$ BI & Yes & Same & Same & Same \\
\hline
\end{tabular}

a. Determined significance value from Table 27.

b. Same by default since the expected significance value is inconclusive.

\section{Comparison with Kazakhstan using the Simplified TAM Model}

The first comparison using the simplified TAM model looked at the number of significant paths for each TAM analysis in Kazakhstan. Table 32 shows that one path was not significant in each analysis of Survey I, II, and III. The first comparison failed, which indicated that the TAM factors in Kazakhstan could differ from those in developed countries

The next assessment examined which paths were not significant, which revealed that the PEOU $\rightarrow$ BI path for each analysis in this project was not significant. According 
to Table 30, this path is almost always significant in developed countries except for in one study. King and He's (2006) analysis demonstrate that path PEOU $\rightarrow$ BI showed significance in 37 out of 67 studies, which indicates that the studies used in this project had a higher significance rate. The TAM results from this study show that the path was consistently insignificant. Based on the second comparison, the factors in Kazakhstan differ from those in developed countries.

The conclusion of the subjective comparison using the simplified version of TAM is that the TAM factors of PU, PEOU, and BI in Kazakhstan are different from the factors in developed countries. The analysis shows that the PEOU $\rightarrow$ BI path is the contributor to this conclusion.

\section{Table 32: $\quad$ Comparison of Significant Values (Simplified TAM)}

\begin{tabular}{|c|c|c|c|c|}
\hline Path & $\begin{array}{l}\text { Is Significance } \\
\text { Expected? }{ }^{\text {a }}\end{array}$ & Survey I Results & $\begin{array}{c}\text { Survey II } \\
\text { Results }\end{array}$ & $\begin{array}{c}\text { Survey III } \\
\text { Results }\end{array}$ \\
\hline $\mathrm{PEOU} \rightarrow \mathrm{PU}$ & Yes & Same & Same & Same \\
\hline $\mathrm{PEOU} \rightarrow \mathrm{BI}$ & Yes & Different & Different & Different \\
\hline $\mathrm{PU} \rightarrow \mathrm{BI}$ & Yes & Same & Same & Same \\
\hline
\end{tabular}

a. Determined significance value from Table 29, supported by King and He (2006)

\section{Learning Management System Objective Data}

There were two sets of analytical data. The first set was from the 47 Englishspeaking students who used Moodle as an integral part of their course. The second set was from a separate Moodle instance set up for students and other instructors to access the materials without logging on. All the other ICT instructors and students used the second server to access ICT course resources, such as online presentations, videos, and lab assignments. Mostly, these students used it to access the ICT labs because they lacked a sufficient level of English to benefit from the online resources. The course instructor's 
data were omitted from the datasets most of the time. Therefore, the data in this section are representative of the students' behavior.

These findings demonstrated that the English-speaking students were frequent users of Moodle. The students were required to use Moodle to a certain degree, but most of the students used Moodle beyond the expectation. The most substantial evidence of this is that the students chose to use Moodle outside of laboratory times, primarily in the evenings. Next, mobile users accounted for $32 \%$ of all visits and $20 \%$ of the actions. The guest-access Moodle instance showed similar results.

\section{English-Speaking Course Analytics}

The analytical data recorded that the 47 students who were enrolled in the English-speaking class opened the ICT course homepage in Moodle approximately 7,200 times during the semester. Figure 12 shows the graph of when the students visited Moodle throughout the semester. Each student opened the course page nine times per week on average, based on a 16-week semester and a week for final exams. Another course that only contained practice quizzes for the final exam was created late in the semester for other ICT students, which they opened less than 200 times. Combined, these students viewed approximately 43,500 pages within a Moodle course. The top four categories described below account for $93.2 \%$ of the visits inside of a Moodle course.

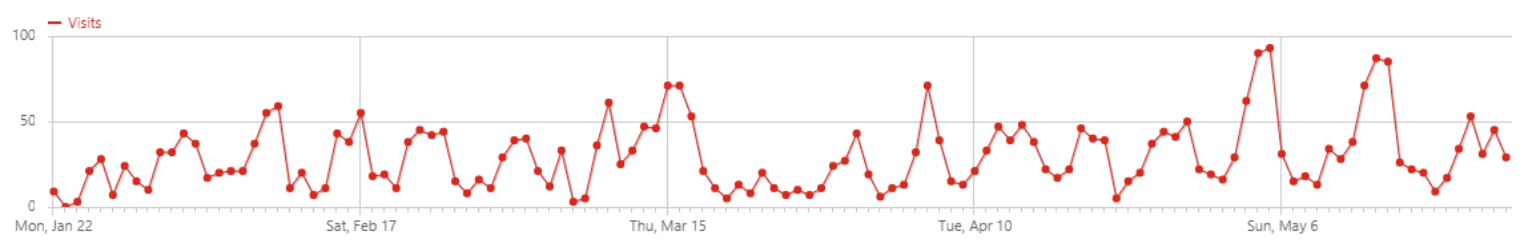

Figure 12: Visits Over Time (Researcher's students) 
The most popular category in Moodle that the students visited once logged on was online quizzes, with 28,710 views or $66.2 \%$ of the course activity. Quizzes were given online and in-class to encourage the students to view the materials before class, both of which were graded. The students who passed the quizzes in Moodle performed well on the quizzes during class time. The quizzes in Moodle were configured to accept the grade with the best attempt, so the students took them multiple times until they had their desired result. The quizzes contained the pertinent information that the students should learn to pass the course successfully.

The students visited the assignment category the second most frequently, with 6,154 views or $14.2 \%$ of the activity. The assignments were predominately the ICT labs that the students completed during the scheduled labs or on their own time.

Third, was the forum category with 4,063 views or $9.4 \%$ of the online activity. These results were surprising because the students seemed to resist engaging in forum activities. These logged visits were predominately views of existing posts because most of the forum assignments resulted in a participation rate of less than half of the students. One forum with high participation was the extra credit activity to celebrate Women's Day. The announcements forum was the fourth most popular, which was a place for the instructor to post assignments, news, changes, and so on. The view count does not accurately capture the rating because the announcements were also delivered to the students' email addresses unless they opted out. It is likely that many students chose to use only the email and not open the announcement in Moodle unless it contained links for an assignment. 
Finally, the category for web pages had 2,526 views or 5.8\%. Pages in Moodle were used to place content such as online presentations, videos with transcripts, some lab instructions, or specific content related to the module. The online presentations or videos only show up in the seventh and ninth page ranking. After removing the other top pages, the online slideshow and video pages accounted for 1,239 visits or $1.8 \%$ of the views. This number is surprisingly low, but it only partially represents the actual number. The module section of the course page contained direct links to the video playlists. The direct links to a Google Document containing the transcript, video links, and presentation links were also in the module section. The video analytics show that the videos were viewed a total 694 times, but the granularity of views was not known. The students clicked the Google-shorted links 948 times, but this number includes the links to the laboratory instructions located in Google Drive. The number of presentation or video views is unknown without an in-depth analysis of which links were clicked.

Some insightful analytics to mention that relate to Moodle's administrative features are grades and messaging. Students viewed their grades 1,977 times or 42 times on average per user, with spikes following the mid-term reports and the final exam. Moodle's messaging feature was a surprise because the students started to use it on their own. Even though they had the instructor's WhatsApp number and email address, the students naturally used messages in Moodle as the primary way to contact their instructor. This behavior was unexpected because WhatsApp is very popular and widely used. At the end of the semester, the students accessed the messages page 617 times.

The students opened the ICT course in Moodle all hours of the day. The most popular times for students to enter it were three hours between 21:00 and 00:00. The 
daytime hours between 08:00 and 15:00 showed consistent times that they logged on. However, the times when the students performed the most actions were at 09:00 (11.1\%), 00:00 (10.6\%), and then at 15:00 (8.3\%). Those three times account for 30\% of all page views in Moodle. Lab times for students were once a week between 08:00 and 09:45 and then from 12:50 to $15: 45$.

The students accessed Moodle $62 \%$ of the time with a desktop or laptop and $37 \%$ of the time using a smartphone. Each device had similar actions per visit at 25.1 for a desktop and 23 for a smartphone. The operating systems used by students were Windows (56.7\%), Android (21.7\%), iOS (10.3\%) and MacOS (2.3\%).

\section{$\underline{\text { Guest-Access Course Analytics }}$}

The other ICT instructors did not want the hassle of having student accounts in Moodle, so a separate Moodle instance was set up with guest-access so their students could access the ICT labs. The course was protected with a single password to discourage uninvited guests from accessing the content. Additionally, some instructors opted to download the labs from Google Drive and distribute the files using a flash drive. The instructors had access to the content on Google Drive, but some of them chose to view the content in Moodle. Therefore, this dataset represents ICT students, ICT instructors, and other unknown users.

In total, the course homepage was accessed 3,694 times. The visitors accessed the presentations in Google Slides 1,089 times. The video pages were accessed 177 times. The laboratory instructions were accessed 2,154 times. The popular times to access the guest-course was from 08:00 to 13:00, which were typical ICT lab times. The next popular times were at night from 19:00 to 23:00. However, 15\% of these visits were from 
outside of Kazakhstan, most likely bots spoofing browsers or unsavory search engines. The users performed 7,876 actions in the course between 10:00 and 14:00. The users performed 3,625 actions during the peak four-hour block in the evening. Figure 13 shows the graph of visits to Moodle during the semester.

The users accessed Moodle $60.8 \%$ of the time with a desktop or laptop and $36.1 \%$ of the time using a smartphone. The desktop users had 9.6 actions per visit, and the smartphone users had 5.4. The operating systems used to access Moodle were Windows (60.9\%), Android (26.4\%), and iOS (11.9\%). There were only two users with the MacOS.

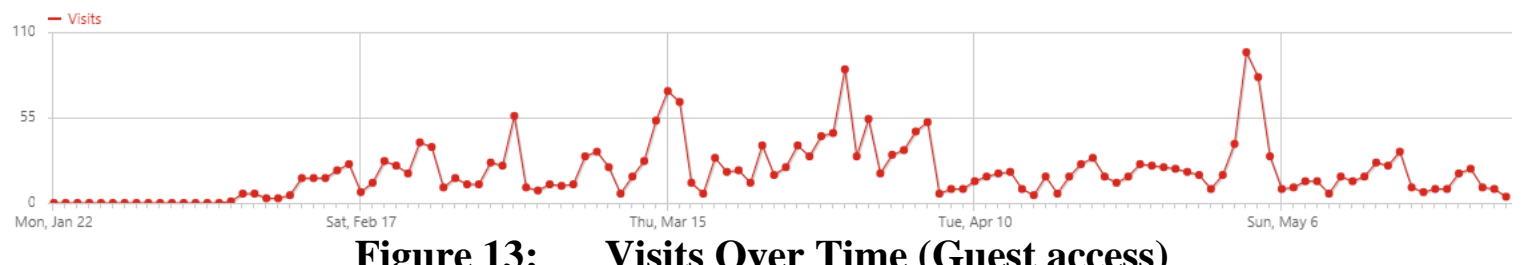

Figure 13: $\quad$ Visits Over Time (Guest access)

\section{Summary of Analysis}

The analysis of this study sought to determine if TAM is an applicable model to predict elearning usage in Kazakhstan, which included a two-part process. Part one generated a TAM profile using data from participants in Kazakhstan. Part two used a subjective comparison to determine if the significant TAM factors in Kazakhstan are the same significant factors in developed countries where researchers have tested and verified the model.

The first step used to create a TAM profile in Kazakhstan was to examine the demographics of the participants, which identified differences in the samples among the surveys. Next, a PCA analysis was performed to determine if the data were suitable for component extraction and path analysis and then to extract the principal components. 
Finally, the path analysis procedure was used to determine the significant correlations between components, path coefficients, and explained variance. Due to inconsistent TAM results, data from the three surveys were used instead of selecting the posttest.

The comparative analysis evaluated the TAM data in Kazakhstan to TAM data in developed countries from educational settings using two criteria. The outcome of this procedure was to determine if the significant factors of PU, PEOU, ATT, and BI in Kazakhstan were the same as those in developed countries. First, the number of significant paths that each TAM study contained were examined in developed countries. Then, non-significant paths were evaluated.

The next chapter interprets the results of the data analysis and answers the research questions. 


\section{CHAPTER SIX: DISCUSSION AND CONCLUSION \\ Introduction}

The purpose of this study was to determine if the technology acceptance model (TAM) is an acceptable method to predict elearning usage in Kazakhstan where the cultural dimensions are different than in North America where the model was developed. In short, both TAM variants revealed differences in Kazakhstan when compared to usages from developed countries. The original TAM model was unreliable, but the simplified version of TAM showed consistency. The original TAM includes the factors of perceived usefulness (PU), perceived ease of use (PEOU), attitude toward using (ATT), and behavioral intention to use (BI). The simplified TAM uses PU, PEOU, and BI.

This chapter evaluates and expounds on the findings in the previous chapter about TAM factors in Kazakhstan before answering the specific research questions because the data revealed unexpected results. Explaining how the various factors that were used to subjectively determine if a significant factor in Kazakhstan was the same in a developed country adds clarity to the research questions. This chapter first addresses the discrepancies among the samples. Next, it summarizes the findings of how TAM performed in Kazakhstan. Third, the chapter evaluates the TAM assumptions in developed countries and compares the suppositions to the findings in Kazakhstan. Fourth, this section examines the cultural aspects of the model and compares expected TAM outcomes based on specific cultural dimensions to the TAM results in Kazakhstan. Next, 
the two research questions are then answered, followed by the limitations and implications of the study. The research questions that this study answers are:

1. Does PU have a stronger influence than PEOU on ATT in Kazakhstan?

2. How do the significant factors of PU, PEOU, ATT, and BI in Kazakhstan compare to the significant factors in developed countries?

\section{Demographics}

The population for this study was typical for the university. The combined samples of Surveys I, II, and II showed that the participants were $52.9 \%$ male, $47.1 \%$ female, $52.9 \%$ preferred the Russian language, and $47.1 \%$ preferred the Kazakh language. The English levels of the participants were $20.7 \%$ A1, 26.4\% A2, $42.5 \%$ B1, and $10.3 \% \mathrm{~B} 2$ or better. There were initial concerns that the population might lack adequate access to the internet or technology skills. These suspicions were unfounded because the data showed that the population had the means to access web-based material and was competent operating mobile or desktop devices. Another uncertainty was how the students would respond to elearning given their bias towards traditional education. The demographics confirmed this suspicion because only $12 \%$ of the population had used an online learning portal before university, but this project's perceptional and behavioral data indicated that the students found value using a learning management system (LMS).

\section{$\underline{\text { Sample Discrepancies }}$}

The three samples illuminated the diversity of the student population, with expected differences between language preference, level of English, and gender. Something unexpected among the samples was the skewed demographic data of either language preference or gender. For example, Survey I showed more male participants, 
Russian preference, and better English while Survey II had more female students, Kazakh preference, and lower English. The lack of a consistent sample might explain the variations among the TAM results. For example, a researcher could theorize that one sample of a predominant characteristic favored PEOU but another preferred PU. Instead of focusing on a single sample or concentrating on the differences in the individual samples, this project centered on the combined result to find the commonalities that allowed the results to show a clearer and less-biased representation of TAM in Kazakhstan. The results established that the ATT construct was not reliable among the population (Table 17). Furthermore, the statistics consistently demonstrated that PEOU had a significant impact on PU and that PU significantly influenced BI (Tables 17 and 18).

\section{Evaluating TAM Factors in Kazakhstan}

\section{$\underline{\text { Summary of TAM Effects }}$}

This study revealed the uncertainty of specific TAM factors and the reliability of other constructs in Kazakhstan. Table 33 summarizes these findings after evaluating both the original and simplified versions of TAM. Firstly, PEOU had a significant direct impact on PU in every instance. Next, PU had a significant direct impact on BI. Thirdly, PU had the most substantial total effect on BI when compared to the other factors (King \& He, 2006). Conversely, PEOU had the least amount of influence on BI among the factors in all instances except one. Fifthly, the data demonstrated that PU captured most of PEOU's effect (King \& He, 2006). Next, ATT was an unreliable construct on both ingress and egress on the original model. The impact on ATT from PU or PEOU 
displayed inconsistency as did the significant effect of ATT on BI. Lastly, all results of the simplified model revealed that the direct effect of PEOU on BI lacked significance.

These findings signify that researchers should cautiously use TAM in Kazakhstan to predict usages of elearning systems. Specifically, these discoveries revealed that PEOU or ATT should not be used to determine BI in Kazakhstan. However, PEOU was highly influential on PU, which captured much of PEOU's influence (King \& He, 2006). The simplified version of TAM showed reliability in this scenario because PU is the vital predictor of BI, but PEOU is a significant influencer on PU.

\section{Table 33: $\quad$ Summary of TAM Effects}

\begin{tabular}{|c|c|c|}
\hline Notable Results & $\begin{array}{c}\text { TAM } \\
\text { Model }\end{array}$ & $\begin{array}{c}\text { Reference } \\
\text { Tables }\end{array}$ \\
\hline 1. The direct impact of PEOU on PU was significant. & Both & 17,18 \\
\hline 2. The direct impact of PU and BI was significant. & Both & 17,18 \\
\hline 3. PU had the largest total effect on BI. & Both & $21-26$ \\
\hline 4. PEOU had the smallest total effect on BI. ${ }^{a}$ & Both & $21-26$ \\
\hline 5. PU captured most of PEOU's effect. & Both & $21-26$ \\
\hline 6. ATT was not a reliable construct. & Original & 17 \\
\hline 7. The direct impact of PEOU on BI was not significant. & Simplified & 18 \\
\hline
\end{tabular}

a. The analysis of original TAM using Survey III data was an exception (Table 25).

\section{Evaluating TAM Expectations from Developed Countries}

Table 34 evaluates the expected TAM results in developed countries and shows the actual results in Kazakhstan. The first expectation was that successful TAM implementations should result in all significant paths. However, Tables 6, 7, and 28 illustrate that it is not typical for a TAM study to result in all significant paths. The results 
of TAM in Kazakhstan showed non-significant paths. Contrary to this expectation, the results in Kazakhstan are comparable to the results in developed countries using this criterion because both contexts contain paths that are not significant. Next, these findings conclusively align with King and He (2006) that PU is the stronger influencer of BI. Thirdly, the data analysis was inadequate to make an affirmative assessment that PEOU was the weakest predictor of actual usage (Turner et al., 2010) because this project lacked a statistical method to compare the perceptive data to the objective data. Theoretically, we could accept this expectation because the perception data in Kazakhstan indicated that PEOU had no statistical significance on BI. Instead, PU captured much of the influence of PEOU (King \& He, 2006). Lastly, the objective data confirmed that BI is an accurate predictor of actual usage (Turner et al., 2010). On average, the students logged onto Moodle nine times a week to take online quizzes, complete ICT labs or other assignments, participate in forums, or view instructional content. However, statistical methods were not used to correlate the behavioral data from the LMS with the TAM perceptional data. 
Table 34: Comparison of Expected TAM Results from Developed Countries

\begin{tabular}{|c|c|}
\hline Expectations in Developed Countries & Results in Kazakhstan \\
\hline $\begin{array}{l}\text { 1. All TAM paths are significant on } \\
\text { successful TAM implementations. }^{\text {a }}\end{array}$ & $\begin{array}{l}\text { False }^{\mathrm{b}} \text { : Every TAM analysis contained at } \\
\text { least one path that was not significant. }\end{array}$ \\
\hline $\begin{array}{l}\text { 2. } \mathrm{PU} \text { is the stronger influencer of } \mathrm{BI} \\
\text { (King \& He, 2006). }\end{array}$ & $\begin{array}{l}\text { True }^{c} \text { : PU had the strongest influence on BI } \\
\text { when compared to all other factors. }\end{array}$ \\
\hline $\begin{array}{l}\text { 3. PEOU is the weakest predictor of } \\
\text { actual usage (Turner et al., 2010). }\end{array}$ & 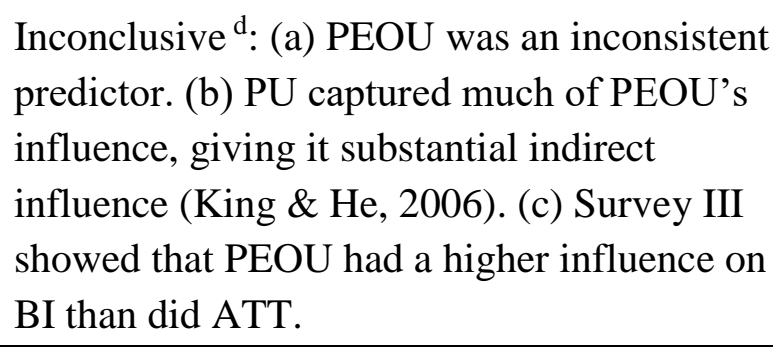 \\
\hline $\begin{array}{l}\text { 4. BI is an accurate predictor of actual } \\
\text { usage (Turner et al., 2010). }\end{array}$ & $\begin{array}{l}\text { True }^{\mathrm{d}} \text { : The objective data confirmed that } \\
\text { students used the LMS. }\end{array}$ \\
\hline
\end{tabular}

a. Data shows that this expectation is unrealistic given unknown factors. See Table 6.

b. This result is inconclusive when compared to other TAM studies. See Table 31.

c. See Tables 21-26.

d. Statistical methods should be used instead of a subjective assessment.

\section{Examining Cultural Data}

The results of the TAM analysis using the simplified model aligned with the expected significant paths given the cultural dimensions of Kazakhstan, as shown in Table 35. Kazakhstan's cultural dimensions that are pertinent to TAM are high power distance (PD), collectivism, and masculinity. Given those three cultural dimensions, the expectations held true since PEOU $\rightarrow$ PU (McCoy et al., 2007) was significant and PEOU $\rightarrow$ BI was not (Huang et al., 2003; McCoy et al., 2007). The prediction of path PU $\rightarrow$ BI was not known because research shows the path is not significant in high PD cultures (Huang et al., 2003; McCoy et al., 2007) but is significant in collectivist and masculine cultures (McCoy et al., 2007). The results showed that PU had a significant 
impact on BI in Kazakhstan. This project did not analyze specific cultural attributes in Kazakhstan that might affect one or more TAM factors.

This research contained cultural biases that could have an impact on a student's intention to use the LMS. First, the foreign ICT teacher was from a low PD country and considerably reduced the PD in the classroom as compared to local instructors. Next, the foreign instructor could have had an unintended positive bias on the students' intention to learn through technology. This learning methodology was new to the students, and they might have been more eager to try it as part of the experience of having an instructor from abroad than from a local instructor. Furthermore, the researcher's opinions would have informed the perceptions of the participants because of subjective norms in a high PD culture (Huang et al., 2003). Third, the sample was from a cross-section of first-year students, not only from the instructor-led class. As mentioned previously, this project did not seek to dissect the factors that were inconsistent across the three surveys but focused on the commonalities. Examining the TAM dimensions using samples from a Kazakh, Russian, and English-speaking class could determine if one or more of these groups have different effects on TAM. Given the limitations and biases of this study, additional research is needed to determine the impact of Kazakhstan's cultural dimensions on TAM. 
Table 35: Expected Significant Paths for Kazakhstan (McCoy et al., 2007)

\begin{tabular}{ccccccl}
\hline \multicolumn{2}{c}{$\mathrm{PU} \rightarrow \mathrm{BI}$} & \multicolumn{2}{c}{ PEOU $\rightarrow \mathrm{BI}$} & \multicolumn{2}{c}{ PEOU $\rightarrow$ PU } & Hofstede's (1980) \\
Expected & Actual & Expected & Actual & Expected & Actual & \multicolumn{1}{c}{ Dimension } \\
\hline No $^{\mathrm{a}}$ & Yes $^{\mathrm{d}}$ & No $^{\mathrm{a}}$ & $\mathrm{No}$ & Yes & Yes & High power distance \\
Yes & Yes & No $^{\mathrm{b}}$ & No & Yes & Yes & High collectivism \\
Yes & Yes & No $^{\mathrm{c}}$ & No & Yes & Yes & High masculinity \\
\hline
\end{tabular}

a. Supported by Huang, Lu, \& Wong (2003).

b. Supported by Abbasi, Tarhini, Elyas, and Shah (2015).

c. Supported by Srite \& Karahanna (2006).

d. Does not confirm that PU $\rightarrow \mathrm{BI}$ is significant in high PD cultures.

This project has shown that Kazakhstan's cultural dimensions of high PD, collectivism, and masculinity are problematic for using TAM to predict technology usage. However, these attributes do not prevent users from technology adoption but change how technology adoption happens. TAM researchers in Saudi Arabia and Jordan, which share Kazakhstan's cultural attributes of high PD and collectivism (Akour, Alshare, Miller, \& Dwairi, 2006; Al-Gahtani, Hubona, \& Wang, 2007; Baker et al., 2010), offer suggestions on technology adoption. Baker et al. (2010) argue that in these cultures, technology adoption comes from the elites of society promoting the technology rather than from the commoners. Akour et al. (2006) echo similar sentiments by suggesting that people of influence in an organization should work diligently to promote the adoption of technologies instead of letting the growth happen organically. Research Question 1: Does PU have a stronger influence than PEOU on ATT in $\underline{\text { Kazakhstan? }}$

The answer to research question one is inconclusive because the data could not prove or disprove the supposition. Table 36 shows that Survey II satisfies the question, 
but Survey I and II do not. As this project discussed previously, the ATT construct was unreliable. Further research is needed to confirm or disprove the argument that PU has a stronger influence than PEOU on ATT in Kazakhstan. This question is an indicator that TAM behaves differently in Kazakhstan, which is explored in research question two.

Table 36: $\quad$ Effects of PU and PEOU on ATT

\begin{tabular}{ccccccc}
\hline & \multicolumn{3}{c}{ Direct Effects on ATT } & \multicolumn{3}{c}{ Total Effects on ATT } \\
Survey & PU $\rightarrow$ & PEOU $\rightarrow$ & Result & PU $\rightarrow$ & PEOU $\rightarrow$ & Result \\
& ATT & ATT & & ATT & ATT & \\
\hline I $^{\mathrm{a}}$ & 0.289 & 0.334 & False & 0.289 & 0.498 & False \\
II $^{\mathrm{b}}$ & 0.441 & 0.073 & True & 0.441 & 0.223 & True \\
III $^{\mathrm{c}}$ & 0.068 & 0.294 & False & 0.068 & 0.321 & False \\
\hline
\end{tabular}

a. Table 21

b. Table 23

c. Table 25

Research Question 2: How do the significant factors of PU, PEOU, ATT, and BI in Kazakhstan compare to the significant factors in developed countries?

\section{Original TAM Perspective}

Most of the significant factors of PU, PEOU, ATT, and BI in Kazakhstan differ from the significant factors in developed countries, as shown in Table 37. Moreover, the original version of TAM in Kazakhstan lacked consistency among the three analyses. None of the analyzed data from the surveys resulted in the same TAM signature. Instead, each analysis displayed one or more non-significant paths that differed from the other results. The comparison for this research question used two criteria, which determined if the path is significant and then if the path should be significant. Only the paths of PEOU $\rightarrow$ PU and PU $\rightarrow$ BI were the same in Kazakhstan as in developed countries. Paths 
$\mathrm{PEOU} \rightarrow \mathrm{ATT}$ and PU $\rightarrow$ ATT consistently showed that they differed. Path ATT $\rightarrow$ BI was inconclusive because it lacked uniformity among the analyses.

A common element to the inconsistent results was the ATT component. When the analyses omitted this factor, TAM performed reliably. This paper did not seek to identify the problems with the ATT construct but offered suppositions instead. First, the analysis revealed discrepancies in the samples that could have produced the differing results. The samples were representative of the research population, but two samples had language and gender bias, and the third survey suffered from low-quality responses. Next, the students found the elearning system more useful if they could use it easily. This research supports the conclusion that PEOU is a significant contributor to BI, but indirectly through PU (King \& He, 2006; Lee et al., 2005). Lastly, the students' attitude about the elearning system was inconsequential regarding their intention to use it because of other factors, such as the impact of cultural differences (Zakour, 2004) on subjective norms (S. Y. Park, 2009; Venkatesh \& Davis, 2000). Research supports that student attitudes toward an elearning system do not automatically transfer to actual system use without a requirement to do so (Ngai et al., 2007). A different perspective on Ngai et al.'s findings is that a user intends to use the system regardless of attitude because of a requirement ( $\mathrm{S}$. A. Brown et al., 2002) or because of cultural influences (Huang et al., 2003; S. Y. Park, 2009; Venkatesh \& Davis, 2000).

A subtle but notable distinction in Kazakhstan's TAM results were the factors that were not significant and those that were. Most TAM studies in developed countries had at least one non-significant path, which was usually $\mathrm{PU} \rightarrow \mathrm{BI}$ or ATT $\rightarrow$ BI (Tables 27 and 28). This project's analyses showed that $\mathrm{PU} \rightarrow \mathrm{BI}$ was always significant and ATT $\rightarrow \mathrm{BI}$ 
was significant every time except once (Table 17). Contrarily, PU $\rightarrow$ ATT and PEOU $\rightarrow$ ATT were significant in developed countries frequently (Tables 6, 7, and 27). In Kazakhstan, these two paths were significant once in the three datasets (Table 17). This subtle peculiarity of which paths were or were not significant demonstrated that factors considered meaningful in Kazakhstan might not be pertinent in developed countries.

This research question comparing TAM in Kazakhstan to developed countries leaves many questions unanswered that further research should address. First, what is the TAM signature in Kazakhstan? The project resulted in three unique outcomes. Answering this question can produce an expected outcome or baseline for future TAM studies. Next, what precisely are the contributing factors that affected the TAM results? This project lacked the sample size necessary to use the TAM 2 model (Venkatesh \& Davis, 2000) or TAM 3 (Venkatesh \& Bala, 2008). Additionally, there is a lack of research on the impact of cultural dimensions using the original TAM model. Researchers have verified the effects of PU and PEOU on BI (McCoy et al., 2007) but not on ATT. Understanding how the dimensions of high PD, collectivism, and masculinity affect a user's attitude can improve the predictivity or reliability of using TAM in Kazakhstan. 
Table 37: Comparison of Paths (Original TAM)

\begin{tabular}{cccc}
\hline & \multicolumn{2}{c}{ TAM Results in Kazakhstan } & \\
\cline { 2 - 3 } Path & Significant Result $^{\mathrm{a}}$ & Is Significance Crucial? $^{\mathrm{b}}$ & Conclusion \\
\hline $\mathrm{PEOU} \rightarrow$ PU & Significant & Yes & Same \\
$\mathrm{PEOU} \rightarrow$ ATT & Not Significant & Yes & Different \\
$\mathrm{PU} \rightarrow$ ATT & Not Significant & Yes & Different \\
$\mathrm{PU} \rightarrow$ BI & Significant & No & Same \\
$\mathrm{ATT} \rightarrow$ BI & Inconclusive $^{\mathrm{c}}$ & Inconclusive & Inconclusive \\
\hline
\end{tabular}

a. Table 17.

b. Tables 27, 28 .

c. Lacked significance in one result; no result had a significance level of $p<0.01$.

\section{$\underline{\text { Simplified TAM Perspective }}$}

The significant factors of PU, PEOU, and BI in Kazakhstan had one difference from the significant factors in developed countries, as shown in Table 38. Unlike the original version, the simplified variant of TAM in Kazakhstan showed consistency among the analyses. All three analyses resulted in the same TAM signature. Specifically, paths of PEOU $\rightarrow$ PU and PU $\rightarrow$ BI were significant, but PEOU $\rightarrow$ BI was not. These results were expected given the cultural dimensions in Kazakhstan (Table 35), as the paper has previously discussed.

This project did not seek to understand the importance of a user's attitude in using an elearning system in Kazakhstan. Consequently, additional research is needed to determine how ATT influences BI. Furthermore, research needs to confirm if the results of the simplified TAM model from this study are consistent. 
Table 38: Comparison of Paths (Simplified TAM)

\begin{tabular}{|c|c|c|c|}
\hline \multirow[b]{2}{*}{ Path } & \multicolumn{2}{|c|}{ TAM Results in Kazakhstan } & \multirow[b]{2}{*}{ Conclusion } \\
\hline & Significant Result ${ }^{\mathrm{a}}$ & Is Significance Crucial? ${ }^{\mathrm{b}}$ & \\
\hline $\mathrm{PEOU} \rightarrow \mathrm{PU}$ & Significant & Yes & Same \\
\hline $\mathrm{PEOU} \rightarrow \mathrm{BI}$ & Not Significant $^{c}$ & Yes & Different \\
\hline $\mathrm{PU} \rightarrow \mathrm{BI}$ & Significant & Yes & Same \\
\hline
\end{tabular}

a. Table 18.

b. Tables 29, 30 .

c. Lacked significance in all analyses.

\section{Limitations and Further Research}

The conclusion of this analysis resulted in an answer of the same, different, or inconclusive for each TAM factor but offered no concrete explanation to identify unusual results. The findings of this project have a degree of uncertainty because the data were not consistent among the pre- and posttests. The overarching limitation of this study is the lack of detail required to give insight or clarity to the findings. Consequently, the findings cannot infer anything beyond that differences exist between TAM results in developed countries and the results in Kazakhstan. The differences listed in Tables 37 and 38 need verification in a broader context, and then future research can address the conflicting data. This section examines specific limitations that require additional research.

First, the context of this research was challenging. The variance in the samples contained students who used the LMS differently. The objective data revealed that the students in the researcher's class found additional value in the LMS beyond the required components whereas the other students primarily used it to access laboratory assignments. This project did not have a large enough sample to capture the data required 
to differentiate the results from the English-speaking ICT students to the ICT students studying in other cohorts. The English-speaking students in the researcher's class used Moodle as an integral part of the course whereas the other students used the LMS as a resource or as a gateway to other web resources. Consequently, the study does not represent either group but provides a cross-section of the first-year students' ICT class. On the one hand, the results accurately represent the population. However, there is speculation that the population has deviating perceptions. Therefore, additional research is needed to separately analyze the students who use the LMS as an integral part of the course and those who use it as a resource. Analyzing each group can create accurate TAM profiles of elearning, which can illuminate discrepancies when analyzing all LMS users.

Secondly, the demographics lacked a suitable analysis that could explain the different results from the variations in the population. The surveys for this project treated the population as a single unit and did not seek to analyze specific characteristics. This project collected essential demographic attributes for reporting purposes, but other important aspects were left out that could provide insight. For example, a correlation might form between mobile-first users and those from rural areas, or the data could reveal that external factors affect the perceptions of elearning differently. Therefore, further research is needed to analyze characteristics of the population to reveal insights about the students that could affect their perceptions of elearning.

Thirdly, culture played a significant role in the study, but this analysis did not seek to make conclusions about the cultural dimensions in Kazakhstan. For example, Huang et al. (2003) confirm McCoy et al. (2007) that PU is not a predictable influencer 
on $\mathrm{BI}$ in a high PD culture using the simplified version of TAM. Contrary to their findings, this study reported that PU significantly influenced BI in every instance. McCoy et al. (2007) also revealed that PU $\rightarrow$ BI should be significant in high collectivist and masculine cultures. Since Kazakhstan portrays all three of these attributes, presumably the most robust characteristics determined the significant value of $\mathrm{PU} \rightarrow \mathrm{BI}$, suggesting that PD is less influential. However, this result could be from the impact of an instructor from a lower PD country. The project did not collect the data necessary to determine if $\mathrm{PU} \rightarrow \mathrm{BI}$ should or should not be significant in high PD cultures. This study can only definitively conclude that PU has a significant impact on BI in the local context of this study. Therefore, additional research is needed in Kazakhstan to verify that PU $\rightarrow$ BI is consistently significant and to determine the effects of high PD, collectivism, and masculinity on the impact of PU on BI.

Fourthly, there is speculation that nonresponse bias (Sax, Gilmartin, \& Bryant, 2003) was introduced using an electronic instrument to collect perception data about an elearning system. The survey administrators did not verify if the students completed the survey, and the participants were not offered any incentive to contribute, which is similar to other perception-based studies in educational contexts (Al-Gahtani, 2016; Roca et al., 2006; Tarhini et al., 2014; Yang \& Yoo, 2004). Instead, a link was placed on the LMS with a message asking the students to fill out the survey. The supposition is that students with favorable attitudes towards elearning and ICT were more likely to complete the electronic survey than those with negative opinions or those lacking confident technology skills. The participation rate in the researcher-led class for Survey II was $87 \%$, and Survey III was 72\%, which is larger than Baruch and Holtom's (2008) study that reported 
an average response rate of $52.7 \%$ for individuals who complete voluntary surveys.

Future studies should ensure a higher participation rate to limit nonresponse bias.

Fifthly, a statistical analysis was not performed on the external TAM factors to determine their impact on perceived usefulness because of the sample size being at the lower bounds and the inconsistencies of TAM in Kazakhstan. By performing a latent variable analysis, additional insights could be learned from the observable external components that would help to interpret the perception data from the surveys and add clarity to the project. Researchers should seek to evaluate how specific external factors affect a user's perception of elearning in Kazakhstan.

Finally, this project did not seek to determine the degree to which learner attitudes impact intentions to use an elearning system in Kazakhstan. Instead, research question number two discussed the suppositions to the findings that ATT is an unreliable construct. Therefore, additional research is needed to determine the factors that affect the ATT construct and how ATT impacts BI in Kazakhstan. Understanding these reasons will guide the interpretations of future research using the original TAM model and alleviate the uncertainty that this project encountered. If future studies confirm that ATT is not a valuable construct, then researchers can use the simplified TAM model to predict system use.

\section{Culture Discussion and Implications}

The challenge of researching perceptions in Kazakhstan is locating the commonalities between the different cultures represented. Cultural bias is difficult to avoid due to sample constraints. For example, different geographical locations in 
Kazakhstan have a different percentage of specific ethnicities ("Ethnic demography of Kazakhstan,” 2017) as do specific contexts that contain the research samples.

The existing literature in Kazakhstan regarding Hofstede's (1980) dimensions is contradictory (Ismail \& Ford, 2010; Mukazhanova, 2012) or lacking verification (Chung \& Holdsworth, 2012; Vasile \& Nicolescu, 2016). Some researchers accurately portray the multi-cultural aspect of Kazakhstan yet direct the research to a particular ethnicity. For example, Nezhina and Ibrayeva (2013) build on the diverse cultural influences in Kazakhstan but then use survey questions, such as the "Kazakh people prefer stable and enforceable law system" (2013, p. 354). Their exclusivity of focusing on Kazakhs for the survey questions build a profile for the Kazakh people, but it does not represent the country of Kazakhstan. Other studies include a non-diverse or bias sample while building a country-wide profile. For example, Karibayeva and Kunanbayeva (2017) focused "on measuring Hofstede's PD index for Kazakh culture" using a sample from the Kazakh business community where they "were able to obtain a PD index of 58 for Kazakhstan" (2017, p. 8). Studies that focus on the majority Kazakh ethnicity ignore $34.5 \%$ of the ethnic diversity in Kazakhstan, which the Slavic minorities represent almost $25 \%$ of the population ("Ethnic demography of Kazakhstan," 2017). These two groups are different based on their history, language, and traditions, but a shared culture exists between them. Trying to build a social profile can result in contradictory information based on the diversity of the sample.

Similarly, the data from this project had ethnicity and gender bias. Building profiles for each ethnicity could give insight into creating a simplified cultural profile that represents commonalities of the population instead of focusing on the specifics. For 
example, the various ethnicities in Kazakhstan might treat the ATT construct differently, which could lead to conflicting data. Excluding the contradictory data might provide a TAM profile that is representative of most of the cultures. For culture studies to accurately represent the population in Kazakhstan, researchers should focus either on the specific aspects of each people group or on a broader set of characteristics that the represented sides have in common.

\section{Implications of the Results}

This study has three areas of impact, which are scientific merit, broader impacts, and local impact. The objective of the scientific merit was to determine if TAM is an acceptable method of predicting usage of elearning systems in Kazakhstan. The findings demonstrated that the original version of TAM was unpredictable in Kazakhstan and displayed unexpected results. The results also demonstrated that the simplified version of TAM was a consistent predictor of BI using the PU construct, which absorbs much of PEOU's influence (King \& He, 2006; Lee et al., 2005). This project provides empirical data about student perceptions of an elearning system in Kazakhstan that gives future researchers a starting or comparison point. Furthermore, this study listed limitations that future researchers can consider before conducting similar studies.

The broader impact provides researchers with TAM data from a developing country that has the cultural dimensions of high PD, high masculinity, and high collectivism. This research builds on past studies that evaluated the cultural effects of TAM, which include McCoy et al. (2007), Straub et al. (1997), Huang et al. (2003), Abbasi et al. (2015), and Srite \& Karahanna (2006). This project confirmed the findings of McCoy et al. (2007) that PEOU $\rightarrow$ BI should not have a significant impact and that 
$\mathrm{PEOU} \rightarrow$ PU expects significance. Prior research has not tested the path PU $\rightarrow$ BI with Kazakhstan's cultural dimensions, which resulted in the path being statistically significant. Furthermore, this study calls into question the validity of TAM studies in other developing countries that did not consider the impact of culture. This study confirmed other research that TAM behaves differently depending on cultural values (Abbasi et al., 2015; Huang et al., 2003; McCoy et al., 2007; Sánchez-Franco et al., 2009; Srite \& Karahanna, 2006; Straub et al., 1997).

Finally, this study contributed to the local body of knowledge at a university in East Kazakhstan concerning what their students think about elearning. This data provides pertinent information to the university as they build up their elearning initiative, especially in their LMS usage. This study demonstrated that students found elearning beneficial and actively used an LMS. It revealed which aspects of the LMS the students used frequently and which parts they rarely used. Additionally, the project demonstrated to the administration and faculty how elearning could work at the university and showed evidence of a successful elearning implementation.

Moreover, this project provided the university with a start to their program that is based on empirical data. Following this analysis, others should perform follow-up studies using larger sample sizes to validate this initial study. Furthermore, follow-up studies are needed to determine what precisely the students like or do not like about the LMS. The core part of this study was only able to provide an overall perception of the LMS, without giving much attention to the details of the student perceptions. The LMS behavioral data only showed how the students used the LMS, but the project lacked the qualitative data from the students. 
In conclusion, this study provides data and insights on how TAM performed in the developing country of Kazakhstan that has the cultural dimension of high PD, masculinity, and collectivism. The data revealed that the ATT construct was not dependable, but TAM was reliable using the factors of PEOU, PU, and BI. In Kazakhstan, the effects were not consistent with TAM results in developed countries. Instead, PU was the primary direct influencer of BI with PEOU being highly influential, but indirectly through PU. These insights can guide future TAM research in developing countries. 


\section{REFERENCES}

Abbasi, M. S., Tarhini, A., Elyas, T., \& Shah, F. (2015). Impact of individualism and collectivism over the individual's technology acceptance behaviour: A multigroup analysis between Pakistan and Turkey. Journal of Enterprise Information Management, 28(6), 747-768.

Abdi, H., \& Williams, L. J. (2010). Principal component analysis. Wiley Interdisciplinary Reviews: Computational Statistics, 2(4), 433-459.

Abdiraiymova, G. S., Burkhanova, D. K., \& Kenzhakimova, G. A. (2012). Higher education in Kazakhstan: New opportunities and problems of crystallization of the middle strata status. In WASET international conference proceedings (Vol. 71).

Agarwal, R., \& Karahanna, E. (2000). Time flies when you're having fun: Cognitive absorption and beliefs about information technology usage. MIS Quarterly, 665694.

Agarwal, R., \& Prasad, J. (1999). Are individual differences germane to the acceptance of new information technologies? Decision Sciences, 30(2), 361-391.

Agbo, I. S. (2015). Factors influencing the use of information and communication technology (ICT) in teaching and learning computer studies in Ohaukwu local government area of Ebonyi State-Nigeria. Journal of Education and Practice, $6(7), 71-86$. 
Aggarwal, D. (2009). Role of E-Learning in a developing country like India. In 3rd National Conference (pp. 26-27).

Aixia, D., \& Wang, D. (2011). Factors influencing learner attitudes toward E-learning and development of E-learning environment based on the integrated E-learning platform. International Journal of E-Education, e-Business, e-Management and eLearning, 1(3), 264-68.

Ajzen, I., \& Fishbein, M. (1975). Belief, attitude, intention and behavior: An introduction to theory and research. Reading, MA: Addison-Wesley.

Ajzen, I., \& Fishbein, M. (1980). Understanding attitudes and predicting social behavior. Englewood Cliffs, NJ: Prentice-Hall.

Ajzen, I., \& Fishbein, M. (2005). The influence of attitudes on behavior. In D. Albarracin, B. T. Johnson, \& M. P. Zanna (Eds.), The handbook of attitudes (pp. 173-221). New York, NY: Psychology Press.

Akour, I., Alshare, K., Miller, D., \& Dwairi, M. (2006). An exploratory analysis of culture, perceived ease of use, perceived usefulness, and internet acceptance: The case of Jordan. Journal of Internet Commerce, 5(3), 83-108.

Al-Adwan, Ahmad, \& Smedley, J. (2012). Implementing e-learning in the Jordanian higher education system: Factors affecting impact. International Journal of Education and Development Using Information and Communication Technology, $8(1), 121$.

Al-Adwan, Amer, Al-Adwan, A., \& Smedley, J. (2013). Exploring students acceptance of e-learning using Technology Acceptance Model in Jordanian universities. 
International Journal of Education and Development Using Information and Communication Technology, 9(2), 4.

Al-Ajlan, A., \& Zedan, H. (2008). Why Moodle. In Proceedings of the 12th IEEE international workshop on future trends of distributed computing system (FTDCS'08) (pp. 58-64). Los Alamitos, CA: IEEE.

Alexander, S. (2001). E-learning developments and experiences. Education \& Training, 43(4/5), 240-248.

Al-Gahtani, S. S. (2001). The applicability of TAM outside North America: An empirical test in the United Kingdom. Information Resources Management Journal, 14(3), 37.

Al-Gahtani, S. S. (2016). Empirical investigation of e-learning acceptance and assimilation: A structural equation model. Applied Computing and Informatics, 12(1), 27-50.

Al-Gahtani, S. S., Hubona, G. S., \& Wang, J. (2007). Information technology (IT) in Saudi Arabia: Culture and the acceptance and use of IT. Information \& Management, 44(8), 681-691.

Alharbi, S., \& Drew, S. (2014). Using the technology acceptance model in understanding academics' behavioural intention to use learning management systems. International Journal of Advanced Computer Science and Applications (IJACSA), $5(1)$.

Alier, M. F., Guerrero, M. J. C., Gonzalez, M. A. C., Penalvo, F. J. G., \& Severance, C. (2010). Interoperability for LMS: The missing piece to become the common place 
for e-learning innovation. International Journal of Knowledge and Learning, 6(23), 130-141.

Ambient Insight. (2014). Ambient Insight regional report: The 2013-2018 Asia self-paced elearning market. Ambient Insight.

Anderson, T. (2004). Towards a theory of online learning. Theory and Practice of Online Learning, 2, 109-119.

Ardichvili, A., \& Kuchinke, K. P. (2002). Leadership styles and cultural values among managers and subordinates: A comparative study of four countries of the former Soviet Union, Germany, and the US. Human Resource Development International, 5(1), 99-117.

Aydin, C. H., \& Tasci, D. (2005). Measuring readiness for e-learning: reflections from an emerging country. Journal of Educational Technology \& Society, 8(4).

Baker, E. W., Al-Gahtani, S. S., \& Hubona, G. S. (2010). Cultural impacts on acceptance and adoption of information technology in a developing country. Journal of Global Information Management (JGIM), 18(3), 35-58.

Baruch, Y., \& Holtom, B. C. (2008). Survey response rate levels and trends in organizational research. Human Relations, 61(8), 1139-1160.

Bentler, P. M., \& Chou, C.-P. (1987). Practical issues in structural modeling. Sociological Methods \& Research, 16(1), 78-117.

Bessadok, A., \& Abdulsalam, A. (2016). Mining student's belief based on e-learning system readiness. International Journal of Information and Education Technology, 10, 6-13. 
Bhuasiri, W., Xaymoungkhoun, O., Zo, H., Rho, J. J., \& Ciganek, A. P. (2012). Critical success factors for e-learning in developing countries: A comparative analysis between ICT experts and faculty. Computers \& Education, 58(2), 843-855.

Blackboard Inc. (2006, March 15). Retrieved June 4, 2017, from http://archive.li/s35zs

Borotis, S., \& Poulymenakou, A. (2004). E-learning readiness components: Key issues to consider before adopting e-learning interventions. In E-Learn: World Conference on E-Learning in Corporate, Government, Healthcare, and Higher Education (pp. 1622-1629). Association for the Advancement of Computing in Education (AACE).

Bro, R., Kjeldahl, K., Smilde, A. K., \& Kiers, H. A. L. (2008). Cross-validation of component models: A critical look at current methods. Analytical and Bioanalytical Chemistry, 390(5), 1241-1251.

Brown, I. T. (2002). Individual and technological factors affecting perceived ease of use of web-based learning technologies in a developing country. The Electronic Journal of Information Systems in Developing Countries, 9.

Brown, S. A., Massey, A. P., Montoya-Weiss, M. M., \& Burkman, J. R. (2002). Do I really have to? User acceptance of mandated technology. European Journal of Information Systems, 11(4), 283-295.

Cavus, N. (2013). Selecting a learning management system (LMS) in developing countries: Instructors' evaluation. Interactive Learning Environments, 21(5), 419437.

Chapnick, S. (2000). Are you ready for e-learning? 
Chen, H., Rong, W., Ma, X., Qu, Y., \& Xiong, Z. (2017). An extended technology acceptance model for mobile social gaming service popularity analysis. Mobile Information Systems, 2017.

Cheng, B., Wang, M., Mørch, A. I., Chen, N.-S., \& Spector, J. M. (2014). Research on elearning in the workplace 2000-2012: A bibliometric analysis of the literature. Educational Research Review, 11, 56-72.

Choi, B., \& Baek, Y. (2011). Exploring factors of media characteristic influencing flow in learning through virtual worlds. Computers \& Education, 57(4), 2382-2394.

Christensen, E. (2013). Technology acceptance model. In E. Kessler (Ed.), Encyclopedia of Management Theory (pp. 829-831). Thousand Oaks, CA, United States: SAGE Publications, Inc.

Chung, K.-C., \& Holdsworth, D. K. (2012). Culture and behavioural intent to adopt mobile commerce among the Y Generation: comparative analyses between Kazakhstan, Morocco and Singapore. Young Consumers, 13(3), 224-241.

Chuttur, M. Y. (2009). Overview of the technology acceptance model: Origins, developments and future directions. Working Papers on Information Systems, 9(37), 9-37.

Coates, H., James, R., \& Baldwin, G. (2005). A critical examination of the effects of learning management systems on university teaching and learning. Tertiary Education and Management, 11, 19-36.

Conde, M. Á., García-Peñalvo, F. J., Rodríguez-Conde, M. J., Alier, M., Casany, M. J., \& Piguillem, J. (2014). An evolving learning management system for new 
educational environments using 2.0 tools. Interactive Learning Environments, $22(2), 188-204$.

Costello, A. B., \& Osborne, J. W. (2005). Best practices in exploratory factor analysis: Four recommendations for getting the most from your analysis. Practical Assessment Research \& Evaluation, 10(7).

Creswell, J. W. (2014). Educational research: Planning, conducting, and evaluating quantitative and qualitative research (5th ed.). Upper Saddle River, NJ: Pearson.

Dalayeva, T. (2013). The e-learning trends of higher education in Kazakhstan. ProcediaSocial and Behavioral Sciences, 93, 1791-1794.

Davis, F. D. (1989). Perceived usefulness, perceived ease of use, and user acceptance of information technology. MIS Quarterly, 319-340.

Davis, F. D. (1993). User acceptance of information technology: System characteristics, user perceptions and behavioral impacts. International Journal of Man-Machine Studies, 38(3), 475-487.

Davis, F. D., Bagozzi, R. P., \& Warshaw, P. R. (1989). User acceptance of computer technology: A comparison of two theoretical models. Management Science, 35(8), 982-1003.

Davis Jr, F. D. (1986). A technology acceptance model for empirically testing new enduser information systems: Theory and results. Massachusetts Institute of Technology. 
de la Sablonnière, R., Taylor, D. M., \& Sadykova, N. (2009). Challenges of applying a student-centered approach to learning in the context of education in Kyrgyzstan. International Journal of Educational Development, 29(6), 628-634.

Dias, S. B., \& Diniz, J. A. (2014). Towards an enhanced learning management system for blended learning in higher education incorporating distinct learners' profiles. Educational Technology \& Society, 17(1), 307-319.

Dishaw, M. T., \& Strong, D. M. (1999). Extending the technology acceptance model with task-technology fit constructs. Information \& Management, 36(1), 9-21.

Docebo. (2016). Elearning market trends and forecast 2017-2021. Retrieved from https://www.docebo.com/resource/elearning-market-trends-and-forecast-20172021/

Downes, S. (2005). E-learning 2.0. Elearn Magazine, 2005(10), 1.

Du, Z., Fu, X., Zhao, C., Liu, Q., \& Liu, T. (2013). Interactive and collaborative elearning platform with integrated social software and learning management system. In W. Lu, G. Cai, W. Liu, \& W. Xing (Eds.), Proceedings of the 2012 International Conference on Information Technology and Software Engineering (pp. 11-18). Heidelberg: Springer-Verlag Berlin Heidelberg.

Duncan, O. D. (1966). Path analysis: Sociological examples. American Journal of Sociology, 72(1), 1-16.

Edutechnica. (2017, March 12). Retrieved June 4, 2017, from http://edutechnica.com/2017/03/12/lms-data-spring-2017-updates/ 
Efron, B. (1982). The jackknife, the bootstrap and other resampling plans. Philadelphia, PA: SIAM.

e-Learning. (2013). Retrieved October 24, 2017, from https://e.edu.kz/en/

Ethnic demography of Kazakhstan. (2017). In Wikipedia. Retrieved from https://en.wikipedia.org/wiki/Ethnic_demography_of_Kazakhstan

Fink, A. (2016). How to conduct surveys: A step-by-step guide (6th ed.). Thousand Oaks, CA: SAGE Publications.

Ford, J. K., MacCallum, R. C., \& Tait, M. (1986). The application of exploratory factor analysis in applied psychology: A critical review and analysis. Personnel Psychology, 39(2), 291-314.

Friedman, B. (1996). Value-sensitive design. Interactions, 3(6), 16-23.

Friesen, N. (2009). Re-thinking e-learning research: Foundations, methods, and practices (Vol. 333). New York, NY: Peter Lang.

García-Peñalvo, F. J., Conde, M. Á., Alier, M., \& Casany, M. J. (2011). Opening learning management systems to personal learning environments. Journal of Universal Computer Science, 17(9), 1222-1240.

Garrison, D. R., \& Kanuka, H. (2004). Blended learning: Uncovering its transformative potential in higher education. The Internet and Higher Education, 7(2), 95-105.

Garson, G. D. (2008). Path analysis. Statnotes: Topics in Multivariate Analysis. Retrieved from http://www.academia.edu/download/39627014/Garson_2008_PathAnalysis.pdf 
Georgouli, K., Skalkidis, I., \& Guerreiro, P. (2008). A framework for adopting LMS to introduce e-learning in a traditional course. Educational Technology \& Society, 11(2), 227-240.

Gilbert, J., Morton, S., \& Rowley, J. (2007). e-Learning: The student experience. British Journal of Educational Technology, 38(4), 560-573.

Greenberg, L. (2002). LMS and LCMS: What's the difference. Learning Circuits, 31(2).

Gulati, S. (2008). Technology-enhanced learning in developing nations: A review. The International Review of Research in Open and Distributed Learning, 9(1), 1-16.

Gunga, S. O., \& Ricketts, I. W. (2007). Facing the challenges of e-learning initiatives in African universities. British Journal of Educational Technology, 38(5), 896-906.

Han, I., \& Shin, W. S. (2016). The use of a mobile learning management system and academic achievement of online students. Computers \& Education, 102, 79-89.

Hartwick, J., \& Barki, H. (1994). Explaining the role of user participation in information system use. Management Science, 40(4), 440-465.

Hashim, H., \& Tasir, Z. (2014). E-learning readiness: A literature review. In Teaching and Learning in Computing and Engineering (LaTiCE), 2014 International Conference on (pp. 267-271). IEEE.

Hofstede, G. (1980). Culture's consequences: International differences in work-related values. Beverly Hills, CA: Sage.

Hofstede, G. (1984). Culture's consequences: International differences in work-related values. Thousand Oaks, CA: Sage. 
Hofstede, G. (1991). Cultures and organizations: Software of the mind. London: McGraw-Hill.

Hofstede, G. (1997). Cultures and organizations: Software of the mind. London: McGraw-Hill.

Hofstede, G. (2011). Dimensionalizing cultures: The Hofstede model in context. Online Readings in Psychology and Culture, 2(1), 8.

Holsapple, C. W., \& Lee-Post, A. (2006). Defining, assessing, and promoting e-learning success: An information systems perspective. Decision Sciences Journal of Innovative Education, 4(1), 67-85.

Horvat, A., Dobrota, M., Krsmanovic, M., \& Cudanov, M. (2015). Student perception of Moodle learning management system: A satisfaction and significance analysis. Interactive Learning Environments, 23(4), 515-527.

House, R. J., Hanges, P. J., Javidan, M., Dorfman, P. W., \& Gupta, V. (2004). Culture, leadership, and organizations: The GLOBE study of 62 societies. Sage publications.

Hrastinski, S. (2008). Asynchronous and synchronous e-learning. Educause Quarterly, $31(4), 51-55$.

Hsu, C.-L., \& Lu, H.-P. (2004). Why do people play on-line games? An extended TAM with social influences and flow experience. Information \& Management, 41(7), $853-868$. 
Huang, L., Lu, M.-T., \& Wong, B. K. (2003). The impact of power distance on email acceptance: Evidence from the PRC. Journal of Computer Information Systems, 44(1), 93-101.

Ismail, K. M., \& Ford, D. L. (2010). Organizational leadership in Central Asia and the Caucasus: Research considerations and directions. Asia Pacific Journal of Management, 27(2), 321-340.

Johnson, R. D., Hornik, S., \& Salas, E. (2008). An empirical examination of factors contributing to the creation of successful e-learning environments. International Journal of Human-Computer Studies, 66(5), 356-369.

Jolliffe, I. T. (2002). Principal component analysis (2nd.). New York, NY: Springer.

Jolliffe, I. T., \& Cadima, J. (2016). Principal component analysis: A review and recent developments. Phil. Trans. R. Soc. A, 374(2065), 20150202.

Josse, J., \& Husson, F. (2012). Selecting the number of components in principal component analysis using cross-validation approximations. Computational Statistics \& Data Analysis, 56(6), 1869-1879.

Kaiser, H. F. (1974). An index of factorial simplicity. Psychometrika, 39(1), 31-36.

Kaiser, H. F., \& Rice, J. (1974). Little jiffy, mark IV. Educational and Psychological Measurement, 34(1), 111-117.

Kamba, M. A. (2009). Problems, challenges and benefits of Implementing e-learning in Nigerian Universities: An empirical study. International Journal of Engineering and Technology, 4(1), 66-69. 
Karibayeva, B., \& Kunanbayeva, S. S. (2017). Power distance and verbal index in Kazakh business discourse. International Journal of Applied Linguistics and English Literature, 6(2), 9-13.

Kazakhstan. (2018). In Wikipedia. Retrieved from https://en.wikipedia.org/wiki/Kazakhstan

Keith, T. Z. (2014). Multiple regression and beyond: An introduction to multiple regression and structural equation modeling (2nd.). New York, NY: Routledge.

Kelly, H. (2014). A path analysis of educator perceptions of open educational resources using the technology acceptance model. The International Review of Research in Open and Distributed Learning, 15(2).

Keung, J., Jeffery, R., \& Kitchenham, B. (2004). The challenge of introducing a new software cost estimation technology into a small software organisation. In Software Engineering Conference, 2004. Proceedings. 2004 Australian (pp. $52-$ 59). IEEE.

King, W. R., \& He, J. (2006). A meta-analysis of the technology acceptance model. Information \& Management, 43(6), 740-755.

Kline, R. B. (2015). Principles and practice of structural equation modeling. New York, NY: Guilford Publications.

Klopping, I. M., \& McKinney, E. (2004). Extending the technology acceptance model and the task-technology fit model to consumer e-commerce. Information Technology, Learning, and Performance Journal, 22(1), 35. 
Kop, R., Fournier, H., \& Mak, J. S. F. (2011). A pedagogy of abundance or a pedagogy to support human beings? Participant support on massive open online courses. The International Review of Research in Open and Distributed Learning, 12(7), 74 93.

Lee, M. K., Cheung, C. M., \& Chen, Z. (2005). Acceptance of Internet-based learning medium: The role of extrinsic and intrinsic motivation. Information \& Management, 42(8), 1095-1104.

Legris, P., Ingham, J., \& Collerette, P. (2003). Why do people use information technology? A critical review of the technology acceptance model. Information \& Management, 40(3), 191-204.

Liaw, S.-S., Chen, G.-D., \& Huang, H.-M. (2008). Users' attitudes toward web-based collaborative learning systems for knowledge management. Computers \& Education, 50(3), 950-961.

Liaw, S.-S., Huang, H.-M., \& Chen, G.-D. (2007). Surveying instructor and learner attitudes toward e-learning. Computers \& Education, 49(4), 1066-1080.

Lin, J. C.-C., \& Lu, H. (2000). Towards an understanding of the behavioural intention to use a web site. International Journal of Information Management, 20(3), 197208.

Lonn, S., \& Teasley, S. D. (2009). Saving time or innovating practice: Investigating perceptions and uses of Learning Management Systems. Computers \& Education, $53(3), 686-694$. 
Lopes, C. T. (2007). Evaluating e-learning readiness in a health sciences higher education institution. In IADIS International Conference ELearning. Citeseer.

Luna, J. M., Castro, C., \& Romero, C. (2017). MDM tool: A data mining framework integrated into Moodle. Computer Applications in Engineering Education, 25(1), $90-102$.

Macfadyen, L. P., \& Dawson, S. (2010). Mining LMS data to develop an "early warning system" for educators: A proof of concept. Computers \& Education, 54(2), 588599.

Mathieson, K. (1991). Predicting user intentions: Comparing the technology acceptance model with the theory of planned behavior. Information Systems Research, 2(3), $173-191$.

Mathieson, K., Peacock, E., \& Chin, W. W. (2001). Extending the technology acceptance model: The influence of perceived user resources. ACM SigMIS Database, 32(3), $86-112$.

McCoy, S., Galletta, D. F., \& King, W. R. (2007). Applying TAM across cultures: The need for caution. European Journal of Information Systems, 16(1), 81-90.

McGill, T. J., \& Klobas, J. E. (2009). A task-technology fit view of learning management system impact. Computers \& Education, 52(2), 496-508.

McGill, T. J., Klobas, J. E., \& Renzi, S. (2014). Critical success factors for the continuation of e-learning initiatives. The Internet and Higher Education, 22, 2436. 
McKay, R. B., Breslow, M. J., Sangster, R. L., Gabbard, S. M., Reynolds, R. W., Nakamoto, J. M., \& Tarnai, J. (1996). Translating survey questionnaires: Lessons learned. New Directions for Evaluation, 1996(70), 93-104.

Means, B., Toyama, Y., Murphy, R., Bakia, M., \& Jones, K. (2009). Evaluation of evidence-based practices in online learning: A meta-analysis and review of online learning studies. US Department of Education.

Montano, D. E., \& Kasprzyk, D. (2008). Theory of reasoned action, theory of planned behavior, and the integrated behavioral model. In K. Glanz, B. K. Rimer, \& K. Viswanath (Eds.), Health behavior and health education: Theory, research, and practice (pp. 67-96). San Francisco, CA: John Wiley \& Sons.

Mosa, A. A., Naz'ri bin Mahrin, M., \& Ibrrahim, R. (2016). Technological aspects of elearning readiness in higher education: A review of the literature. Computer and Information Science, 9(1), 113.

Mott, J. (2010). Envisioning the post-LMS era: The open learning network. Educause Quarterly, 33(1), 1-9.

Mouyabi, J. S. M. (2011). Higher education in the wake of new ICT: Reaping benefits or creating more problems through e-learning? South African Journal of Higher Education, 25(6), 1178-1189.

Mukazhanova, K. (2012). A cross-cultural comparison of leadership choices: Commonalities and differences among female leaders in the United States, Kazakhstan and Sweden (Master of Arts thesis). University of Oregon. Retrieved from https://scholarsbank.uoregon.edu/xmlui/handle/1794/12398 
Mumtaz, S. (2000). Factors affecting teachers' use of information and communications technology: A review of the literature. Journal of Information Technology for Teacher Education, 9(3), 319-342.

Mun, Y. Y., \& Hwang, Y. (2003). Predicting the use of web-based information systems: Self-efficacy, enjoyment, learning goal orientation, and the technology acceptance model. International Journal of Human-Computer Studies, 59(4), 431-449.

Nawaz, A. (2013). Using e-learning as a tool for education for all in developing states. International Journal of Science and Technology Education Research, 4(3), 3846.

Nazarbayev University Graduate School of Education [NUGSE]. (2014). Development of strategic directions for education reforms in Kazakhstan for 2015-2020. Diagnostic report. Astana: Indigo print. Retrieved from https://nur.nu.edu.kz/bitstream/handle/123456789/335/DIAGNOSTIC\%20REPO RT.pdf

Nezhina, T. G., \& Ibrayeva, A. R. (2013). Explaining the role of culture and traditions in functioning of civil society organizations in Kazakhstan. Voluntas: International Journal of Voluntary and Nonprofit Organizations, 24(2), 335-358.

Ngai, E. W., Poon, J. K. L., \& Chan, Y. H. C. (2007). Empirical examination of the adoption of WebCT using TAM. Computers \& Education, 48(2), 250-267.

Ngampornchai, A., \& Adams, J. (2016). Students' acceptance and readiness for Elearning in Northeastern Thailand. International Journal of Educational Technology in Higher Education, 13(1), 34. 
O'Flaherty, J., \& Phillips, C. (2015). The use of flipped classrooms in higher education: A scoping review. The Internet and Higher Education, 25, 85-95.

Oliveira, P. C. de, Cunha, C. J. C. de A., \& Nakayama, M. K. (2016). Learning management systems (LMS) and e-learning management: An integrative review and research agenda. JISTEM-Journal of Information Systems and Technology Management, 13(2), 157-180.

O’Rourke, N., \& Hatcher, L. (2013). A step-by-step approach to using SAS for factor analysis and structural equation modeling. Cary, NC: SAS Institute.

Osborne, J. W., \& Costello, A. B. (2004). Sample size and subject to item ratio in principal components analysis. Practical Assessment, Research \& Evaluation, $9(11), 8$.

Padilla-MeléNdez, A., Del Aguila-Obra, A. R., \& Garrido-Moreno, A. (2013). Perceived playfulness, gender differences and technology acceptance model in a blended learning scenario. Computers \& Education, 63, 306-317.

Paola Torres Maldonado, U., Feroz Khan, G., Moon, J., \& Jeung Rho, J. (2011). Elearning motivation and educational portal acceptance in developing countries. Online Information Review, 35(1), 66-85.

Park, N., Roman, R., Lee, S., \& Chung, J. E. (2009). User acceptance of a digital library system in developing countries: An application of the Technology Acceptance Model. International Journal of Information Management, 29(3), 196-209. 
Park, S. Y. (2009). An analysis of the technology acceptance model in understanding university students' behavioral intention to use e-learning. Educational Technology \& Society, 12(3), 150-162.

Parkes, M., Stein, S., \& Reading, C. (2015). Student preparedness for university elearning environments. The Internet and Higher Education, 25, 1-10.

Perraton, H. (2005). Open and distance learning in the developing world. Routledge.

Psycharis, S. (2005). Presumptions and actions affecting an e-learning adoption by the educational system-Implementation using virtual private networks. European Journal of Open, Distance and E-Learning, 8(2).

Rawstorne, P., Jayasuriya, R., \& Caputi, P. (2000). Issues in predicting and explaining usage behaviors with the technology acceptance model and the theory of planned behavior when usage is mandatory. In Proceedings of the twenty first international conference on Information systems (pp. 35-44). Association for Information Systems.

Roca, J. C., Chiu, C.-M., \& Martínez, F. J. (2006). Understanding e-learning continuance intention: An extension of the Technology Acceptance Model. International Journal of Human-Computer Studies, 64(8), 683-696.

Romero, C., Cerezo, R., Bogarín, A., \& Sánchez-Santillán, M. (2016). Educational process mining: A tutorial and case study using Moodle data sets. In S. ElAtia, D. Ipperciel, \& O. R. Zaiane (Eds.), Data mining and learning analytics: Applications in educational research (1st., pp. 3-28). Hoboken, NJ: John Wiley \& Sons, Inc. 
Romero, C., Espejo, P. G., Zafra, A., Romero, J. R., \& Ventura, S. (2013). Web usage mining for predicting final marks of students that use Moodle courses. Computer Applications in Engineering Education, 21(1), 135-146.

Romero, C., \& Ventura, S. (2010). Educational data mining: A review of the state of the art. IEEE Transactions on Systems, Man, and Cybernetics, Part C (Applications and Reviews), 40(6), 601-618.

Romero, C., \& Ventura, S. (2013). Data mining in education. Wiley Interdisciplinary Reviews: Data Mining and Knowledge Discovery, 3(1), 12-27.

Romero, C., Ventura, S., \& García, E. (2008). Data mining in course management systems: Moodle case study and tutorial. Computers \& Education, 51(1), 368384.

Ruel, E., Wagner III, W. E., \& Gillespie, B. J. (2015). The practice of survey research: Theory and applications. Los Angeles, CA: SAGE Publications.

Saadé, R. G., Nebebe, F., \& Tan, W. (2007). Viability of the "Technology Acceptance Model" in multimedia learning environments: A comparative study. Interdisciplinary Journal of Knowledge and Learning Objects, 3(1), 175-184.

Sánchez-Franco, M. J., Martínez-López, F. J., \& Martín-Velicia, F. A. (2009). Exploring the impact of individualism and uncertainty avoidance in Web-based electronic learning: An empirical analysis in European higher education. Computers \& Education, 52(3), 588-598. 
Sapargaliyev, D. (2011). The organization of mobile learning in higher education of Kazakhstan. Enhancing Learning Through Technology. Education Unplugged: Mobile Technologies and Web 2.0, 63-70.

Sapargaliyev, D. (2012). Development of distance education in Kazakhstan.

Sax, L. J., Gilmartin, S. K., \& Bryant, A. N. (2003). Assessing response rates and nonresponse bias in web and paper surveys. Research in Higher Education, 44(4), 409-432.

Schoonenboom, J. (2014). Using an adapted, task-level technology acceptance model to explain why instructors in higher education intend to use some learning management system tools more than others. Computers \& Education, 71, 247256.

Selim, H. M. (2007). Critical success factors for e-learning acceptance: Confirmatory factor models. Computers \& Education, 49(2), 396-413.

Shroff, R. H., Deneen, C. C., \& Ng, E. M. (2011). Analysis of the technology acceptance model in examining students' behavioural intention to use an e-portfolio system. Australasian Journal of Educational Technology, 27(4).

Sife, A., \& Lwoga, E. (2007). New technologies for teaching and learning: Challenges for higher learning institutions in developing countries. International Journal of Education and Development Using ICT, 3(2).

Singh, H. (2003). Building effective blended learning programs. Educational Technology, 43(6), 51-54. 
Smith, L. I. (2002). A tutorial on principal components analysis. Retrieved from http://www.cs.otago.ac.nz/cosc453/student_tutorials/principal_components.pdf

Srite, M., \& Karahanna, E. (2006). The role of espoused national cultural values in technology acceptance. MIS Quarterly, 679-704.

Stantchev, V., Colomo-Palacios, R., Soto-Acosta, P., \& Misra, S. (2014). Learning management systems and cloud file hosting services: A study on students' acceptance. Computers in Human Behavior, 31, 612-619.

Straub, D., Keil, M., \& Brenner, W. (1997). Testing the technology acceptance model across cultures: A three country study. Information \& Management, 33(1), 1-11.

Suhr, D. (2008). Step your way through path analysis. In Western Users of SAS Software Conference Proceedings.

Suhr, D. D. (2005). Principal component analysis vs. exploratory factor analysis. In SUGI 30 proceedings.

Šumak, B., Heričko, M., Pušnik, M., \& Polančič, G. (2011). Factors affecting acceptance and use of Moodle: An empirical study based on TAM. Informatica, 35(1), 91100.

Tabachnick, B. G., \& Fidell, L. S. (2007). Using multivariate statistics. Boston, MA: Pearson Education Inc.

Tarhini, A., Hone, K., \& Liu, X. (2013). Extending the TAM model to empirically investigate the students' behavioural intention to use e-learning in developing countries. In Science and Information Conference (SAI), 2013 (pp. 732-737). IEEE. 
Tarhini, A., Hone, K., \& Liu, X. (2014). The effects of individual differences on elearning users' behaviour in developing countries: A structural equation model. Computers in Human Behavior, 41, 153-163.

Tarhini, A., Hone, K., Liu, X., \& Tarhini, T. (2017). Examining the moderating effect of individual-level cultural values on users' acceptance of E-learning in developing countries: A structural equation modeling of an extended technology acceptance model. Interactive Learning Environments, 25(3), 306-328.

Teo, T. (2010). A path analysis of pre-service teachers' attitudes to computer use: Applying and extending the technology acceptance model in an educational context. Interactive Learning Environments, 18(1), 65-79.

Teo, T. (2011). Factors influencing teachers' intention to use technology: Model development and test. Computers \& Education, 57(4), 2432-2440.

Teo, T. S., Lim, V. K., \& Lai, R. Y. (1999). Intrinsic and extrinsic motivation in Internet usage. Omega, 27(1), 25-37.

Tselios, N. K., Daskalakis, S., \& Papadopoulou, M. (2011). Assessing the acceptance of a blended learning university course. Educational Technology \& Society, 14(2), 224-235.

Tucker, B. (2012). The flipped classroom: Online instruction at home frees class time for learning. Education Next, 12(1), 82-83.

Turner, M., Kitchenham, B., Brereton, P., Charters, S., \& Budgen, D. (2010). Does the technology acceptance model predict actual use? A systematic literature review. Information and Software Technology, 52(5), 463-479. 
UN/DESA. (2014). World economic situation and prospects 2014: Country classification. Retrieved from http://www.un.org/en/development/desa/policy/wesp/wesp_current/2014wesp_co untry_classification.pdf

United Nations Development Programme. (2016). Human development report 2016: Human development for everyone. Retrieved from http://hdr.undp.org/sites/default/files/2016_human_development_report.pdf

Urdan, T. A., \& Weggen, C. C. (2000). Corporate elearning: Exploring a new frontier.

Vasile, A. C., \& Nicolescu, L. (2016). Hofstede's cultural dimensions and management in corporations. Cross Cultural Management Journal, (9), 35-46.

Venkatesh, V., \& Bala, H. (2008). Technology acceptance model 3 and a research agenda on interventions. Decision Sciences, 39(2), 273-315.

Venkatesh, V., \& Davis, F. D. (1996). A model of the antecedents of perceived ease of use: Development and test. Decision Sciences, 27(3), 451-481.

Venkatesh, V., \& Davis, F. D. (2000). A theoretical extension of the technology acceptance model: Four longitudinal field studies. Management Science, 46(2), $186-204$.

Verbert, K., Duval, E., Klerkx, J., Govaerts, S., \& Santos, J. L. (2013). Learning analytics dashboard applications. American Behavioral Scientist, 57(10), 1500-1509.

Verbert, K., Govaerts, S., Duval, E., Santos, J. L., Van Assche, F., Parra, G., \& Klerkx, J. (2014). Learning dashboards: An overview and future research opportunities. Personal and Ubiquitous Computing, 18(6), 1499-1514. 
Vyas, S., \& Kumaranayake, L. (2006). Constructing socio-economic status indices: How to use principal components analysis. Health Policy and Planning, 21(6), 459468.

Watson, W. R., \& Watson, S. L. (2007). What are learning management systems, what are they not, and what should they become. TechTrends, 51(2), 29.

Westland, J. C. (2010). Lower bounds on sample size in structural equation modeling. Electronic Commerce Research and Applications, 9(6), 476-487.

Williams, B., Onsman, A., \& Brown, T. (2010). Exploratory factor analysis: A five-step guide for novices. Australasian Journal of Paramedicine, 8(3).

Wold, S. (1978). Cross-validatory estimation of the number of components in factor and principal components models. Technometrics, 20(4), 397-405.

Wold, S., Esbensen, K., \& Geladi, P. (1987). Principal component analysis. Chemometrics and Intelligent Laboratory Systems, 2(1-3), 37-52.

Wu, J.-H., Tennyson, R. D., \& Hsia, T.-L. (2010). A study of student satisfaction in a blended e-learning system environment. Computers \& Education, 55(1), 155-164.

Yang, H., \& Yoo, Y. (2004). It's all about attitude: revisiting the technology acceptance model. Decision Support Systems, 38(1), 19-31.

Zakour, A. B. (2004). Cultural differences and information technology acceptance. In Proceedings of the 7th annual conference of the Southern association for information systems (pp. 156-161). 
APPENDIX A

Referenced TAM Questions 


\section{$\underline{\text { Perceived Usefulness of elearning (PU) }}$}

1. PU1: Using the elearning system helps me learn my course content (PadillaMeléNdez et al., 2013; S. Y. Park, 2009; Roca et al., 2006)

2. PU2: Using the elearning system makes it easier to study the course content (Alharbi \& Drew, 2014; S. Y. Park, 2009)

3. PU3: Using the elearning system makes it easier to learn in university (Agarwal \& Prasad, 1999; Ngai et al., 2007)

4. PU4: Using the elearning system helps me organize my learning

5. PU5: Using the elearning system is useful to my learning (Chen et al., 2017; Legris et al., 2003; Padilla-MeléNdez et al., 2013)

6. PU6: Overall, I think that using the elearning system is useful in my studies (McGill \& Klobas, 2009)

\section{$\underline{\text { Perceived Ease of Use of elearning (PEOU) }}$}

1. PEOU1: It is easy for me to become skillful using the elearning system (S. Y. Park, 2009; T. Teo, 2011)

2. PEOU2: I find navigating the elearning system easy (T. Teo, 2011)

3. PEOU3: Overall, the elearning system is easy to use (Ngai et al., 2007)

4. PEOU4: Using the elearning system is easy for me (Roca et al., 2006; T. S. Teo et al., 1999)

5. PEOU5: I find the elearning system easy to use (S. Y. Park, 2009; T. S. Teo et al., 1999; Turner et al., 2010)

6. PEOU6: Learning how to use an elearning system is easy for me (S. Y. Park, 2009)

\section{Attitude Toward Using elearning (ATT)}

1. ATT1: I like to study using an elearning system (Agarwal \& Prasad, 1999; Chen et al., 2017; Padilla-MeléNdez et al., 2013)

2. ATT2: I like the idea of using an elearning system (Alharbi \& Drew, 2014; Šumak et al., 2011)

3. ATT3: I think using an elearning system makes studying more interesting (Šumak et al., 2011)

4. ATT4: Studying through an elearning system is a good idea (Ngai et al., 2007; S. Y. Park, 2009)

5. ATT5: I am positive toward using technology for learning (S. Y. Park, 2009)

6. ATT6: Overall, I like working with the elearning system (Ngai et al., 2007; Šmak et al., 2011; T. Teo, 2011) 


\section{Survey I - English}

Demographics (DEM)

DEM10-DEM12 | Multi-Point Scale: 1=expert, 7=novice

- DEM1: Gender

○ Multiple Choice: M/F

- DEM2: Current class (year in university)

- Multiple Choice: 1st, 2nd, 3rd, 4th, other

- DEM3: Study major

- Short Answer:

- DEM4: Level of English

○ Multiple Choice: A1, A2, B1, B2, C1, C2, other

- DEM5: My mobile phone is connected to the internet of the time

○ Multi-Point Scale: $1=100 \%, 4=50 \% 7=0 \%$

- DEM6: I prefer to browse the web using:

- Multiple Choice: A mobile device or, A computer (laptop or desktop), Both equally

- DEM7: I have the following computers at home

○ Multiple selection: desktop, laptop, tablet, none

- DEM8: My internet connection at home is

- Multiple Choice: fiber optics, DSL, dial-up, 3G/4G modem, other, do not know

- DEM9: How fast do you consider your internet connection at home?

- Multi-Point Scale: 1=very fast, $7=$ very slow

- DEM10: My experience level at using the internet

- DEM11: My experience using a computer (desktop or laptop)

- DEM12: My experience using a smartphone

Previous Elearning Experience (PEX)

PEX1-PEX2 | Multiple Choice: yes, no, do not know

PEX3-PEX7 | Multi-Point Scale: 1=always, 7=never

- PEX1: My high school had a computer lab for the students to use:

- PEX2: My high school had an online learning portal

- PEX3: I used an online learning portal at my high school

- PEX4: I submitted assignments through an online learning portal at my high school 
- PEX5: I accessed course material through an online learning portal at my high school

- PEX6: My teacher posted assignments to an online learning portal at my high school

- PEX7: My high school teacher communicated with us through an online learning portal

Perceived Usefulness of elearning (PU)

PU1-PU6 | Multi-Point Scale: 1=strongly agree, 7=strongly disagree

- PU1: Using the elearning system helps me learn my course content

- PU2: Using the elearning system makes it easier to study the course content

- PU3: Using the elearning system makes it easier to learn in university

- PU4: Using the elearning system helps me organize my learning

- PU5: Using the elearning system is useful to my learning

- PU6: Overall, I think that using the elearning system is useful in my studies

Perceived Ease of Use of elearning (PEOU)

PEOU1-PEOU6 | Multi-Point Scale: 1=strongly agree, 7=strongly disagree

- PEOU1: It is easy for me to become skillful using the elearning system

- PEOU2: I find navigating the elearning system easy

- PEOU3: Overall, the elearning system is easy to use

- PEOU4: Using the elearning system is easy for me

- PEOU5: I find elearning system easy to use

- PEOU6: Learning how to use an elearning system is easy for me

Attitude Toward Using elearning (ATT)

ATT1-ATT6 | Multi-Point Scale: 1=strongly agree, 7=strongly disagree

- ATT1: I like to study using an elearning system

- ATT2: I like the idea of using an elearning system

- ATT3: I think using an elearning system makes studying more interesting

- ATT4: Studying through an elearning system is a good idea

- ATT5: I am positive toward using technology for learning

- ATT6: Overall, I like working with the elearning system 
$\underline{\text { Behavioral Intention towards using elearning (BI) }}$

BI1-BI6 | Multi-Point Scale: 1=strongly agree, 7=strongly disagree

- BI1: I would use an elearning system in my class if my teacher offered it

- BI2: I would want to use an elearning system if my other classes offered it

- BI3: I hope that other courses will use an elearning system

- BI4: I would like to use an elearning system in the future

- BI5: If my other courses had an elearning system, I would use it

- BI6: If an elearning system were not required for this course, I would still want to use it

\section{Survey I - Russian}

\section{Демографические данные (DEM)}

DEM10-DEM12 | Многоуровневая шкала: 1=эксперт, 7= новичок

- DEM1: Пол

○ Варианты ответа: М/Ж, предпочитаю не отвечать

- DEM2: курс (год обучения в университете)

○ Варианты ответа: 1, 2, 3, 4, другой

- DEM3: Профилирующая дисциплина обучения

○ Краткий ответ:

- DEM4: Уровень владения английским языком

○ Варианты ответа: A1, A2, B1, B2, C1, C2, другой

- DEM5: Мой мобильный подсоединен к интернету времени

○ Многоуровневая шкала: $1=100 \%, 4=50 \% 7=0 \%$

- DEM6: Я предпочитаю выходить в интернет через:

○ Варианты ответа: Мобильное устройство или, компьютер (ноутбук или ПК), оба варианта

- DEM7: У меня дома есть такие виды компьютера как

○ Возможны несколько вариантов: ПК, ноутбук, планшет, нет

- DEM8: Интернет соединение у меня дома это

○ Варианты ответа: оптоволокно, DSL, соединение по телефонной линии, 3G/4G модем, другое, не знаю

- DEM9: Насколько быстрое, вы считаете, интернет соединение у вас дома

○ Многоуровневая шкала: 1=очень быстрое, $7=$ =чень медленное

- DEM10: Мой уровень компетентности в использовании интернета

- DEM11: Мой уровень компетентности в использовании компьютера (ПК или ноутбука) 
- DEM12: Мой уровень компетентности в использовании смартфона Предыдущий опыт интернет обучения (PEX)

PEX1-PEX2 | Варианты ответа: да, нет, не знаю

PEX3-РЕХ7 | Многоуровневая шкала: 1=всегда, 7=никогда

- PEX1: В старших классах моей школы ученики могли пользоваться компьютерным классом

- PЕХ2: В старших классах моей школы был образовательный портал

- РЕХЗ: Я пользовался образовательным порталом в старших классах

- PЕX4: Я сдавал домашние задания через образовательный портал в старших классах

- PEX5: Я получал доступ к учебным материалам через образовательный портал в старших классах

- PЕХ6: Мой учитель отправлял мне задания через образовательный портал в старших классах

- PEX7: Мой учитель общался с нами через образовательный портал в старших классах

Оцениваемая практичность интернет обучения (PU)

PU1-PU6 | Многоуровневая шкала: 1=полностью согласен 7=полностью не согласен

- PU1: Использование системы электронного обучения помогает мне изучать содержание учебного курса

- PU2: Использование системы электронного обучения облегчает изучение содержания учебного курса

- PU3: Использование системы электронного обучения облегчает учебу в университете

- PU4: Использование системы электронного обучения помогает мне организовать мое обучение

- PU5: Использование системы электронного обучения полезно для моего обучения

- PU6: В целом, я считаю что использование системы электронного обучения полезно для моей учебы 
Оцениваемая простота использования системы интернет обучения (PEOU)

PEOU1-PEOU6 | Многоуровневая шкала: 1=полностью согласен

$7=$ полностью не согласен

- PEOU1: Для меня просто стать экспертом по использованию системы электронного обучения

- PEOU2: Мне просто ориентироваться в системе электронного обучения

- PEOU3: В целом, система электронного обучения проста в использовании

- PEOU4: Мне просто пользоваться системой электронного обучения

- PEOU5: Я считаю система электронного обучения проста в использовании

- PEOU6: Изучение того, как пользоваться системой электронного обучения просто для меня

Отношение к использованию интернет обучения (АТT)

ATT1-АТТ6 | Многоуровневая шкала: 1=полностью согласен 7=полностью

не согласен

- ATT1: Мне нравится пользоваться системой электронного обучения

- АТT2: Мне нравится идея использования системы электронного обучения

- ATT3: Я считаю, что система электронного обучения делает учебу более интересной

- ATT4: Учебный процесс через систему электронного обучения это хорошая идея

- АТТ5: Я положительно отношусь к использовании технологий в обучении

- ATT6: В целом мне нравиться работать с системой электронного обучения

Намерение использовать электронное обучение (BI)

BI1-BI6 | Многоуровневая шкала: 1=полностью согласен 7=полностью не согласен

- BI1: Я бы использовал систему электронного обучения, если бы мне предложил преподаватель 
- ВІ2: Я бы хотел использовать систему электронного обучения, если бы мне предложили это на занятии

- BI3: Я надеюсь что на других дисциплинах будет использоваться система электронного обучения

- BI4: Я бы хотел использовать систему электронного обучения в будущем

- BI5: Если бы по другим дисциплинам мне предложили использовать систему электронного обучения, я бы согласился

- BI6: Если бы система электронного обучения не была необходимой для данного курса, я бы все равно хотел бы ей пользова

\section{Survey II - English}

\section{Demographics (DEM)}

- DEM1: Gender

- Multiple Choice: M/F, Prefer not to answer

- DEM2: Group

○ Short Answer:

- DEM3: Mr. Hetrick is my instructor.

- Multiple Choice: Y/N, Prefer not to answer

- DEM4: Level of English

- Multiple Choice: A1, A2, B1, B2, C1, C2, other

\section{Technology (TEC)}

TEC1: My mobile phone is connected to the internet

Multi-Point Scale: $1=$ Always, $5=$ never

- TEC2: I prefer to browse the web using:

- Multiple Choice: A mobile device or, A computer (laptop or desktop), Both equally

- TEC3: I have the following computers at home

- Multiple selection: desktop, laptop, tablet, none

- TEC4: My internet connection at home is

- Multiple Choice: fiber optics, DSL, dial-up, 3G/4G modem, other, do not know, I do not have home internet at home

- TEC5: How fast do you consider your internet connection at home?

- Multi-Point Scale: $1=$ very fast, $7=$ very slow

- ETC6: In your opinion, how easy or difficult is for you to use the internet?

- Multi-Point Scale: 1=Extremely easy, $7=$ Extremely difficult 
- TEC7: In your opinion, how easy or difficult is for you to use a computer (desktop or laptop)?

- Multi-Point Scale: 1=Extremely easy, 7=Extremely difficult

- TEC8: In your opinion, how easy or difficult is for you to use a smartphone?

○ Multi-Point Scale: 1=Extremely easy, 7=Extremely difficult

$\underline{\text { Technology Readiness (TR) }}$

TR1-TR7 | Multi-Point Scale: 1=strongly agree, 7=strongly disagree

- TR1: Using a web browser is easy for me

- TR2: I can find what I want on the internet with ease

- TR3: Navigating websites is easy for me

- TR4: I can easily share pictures on social media, such as Instagram, Facebook, OK.ru, or Vk

- TR5: It is easy for me to take a picture and post it to a social media site

- TR6: It is easy for me to create new accounts on websites

- TR7: Playing and controlling media in a web browser or mobile app is easy for me

Learner Readiness (LR)

LR1-LR6 | Multi-Point Scale: 1=strongly agree, 7=strongly disagree

- LR1: I would like to study with the assistance of technology

- LR2: I like the idea of studying online

- LR3: Having online resources makes my studying more interesting

- LR4: I think technology can improve my learning

- LR5: Using technology can help me study

- LR6: To do an assignment, I would rather use an electronic resource (a website or digital journal) than a textbook

\section{Content Readiness (CR)}

CR1-CR6 | Multi-Point Scale: 1=strongly agree, 7=strongly disagree

- CR1: I feel prepared to study using the internet

- CR2: I could learn equally well by listening to a live lecture in a classroom or watching an online video at home

- CR3: I would want to learn both from classroom lectures and from online presentations 
- CR4: I think I could learn more online than listening to lectures in the classroom

- CR5: I think education should include both learning in the classroom and learning online

- CR6: I would prefer if my instructor gave me assignments to do that required the internet

\section{Previous Elearning Experience (PEX)}

PEX1-PEX2 | Multiple Choice: yes, no, do not know

PEX3-PEX7 | Multi-Point Scale: 1=always, 7=never

- PEX1: My high school had a computer lab for the students to use:

- PEX2: My high school had an online learning portal

- PEX3: I used an online learning portal at my high school

- PEX4: I submitted assignments through an online learning portal at my high school

- PEX5: I accessed course material through an online learning portal at my high school

- PEX6: My teacher posted assignments to an online learning portal at my high school

- PEX7: My high school teacher communicated with us through an online learning portal

Perceived Usefulness of elearning (PU)

PU1-PU4 | Multi-Point Scale: 1=strongly agree, 7=strongly disagree

- PU1: Using the elearning system helps me learn my course content

- PU2: Using the elearning system helps me organize my learning

- PU3: Using the elearning system makes it easier to study the course content

- PU4: Using the elearning system is useful to my learning

\section{Perceived Ease of Use of elearning (PEOU)}

PEOU1-PEOU5 | Multi-Point Scale: 1=strongly agree, 7=strongly disagree

- PEOU1: Moodle loads quickly

- PEOU2: Navigating the elearning system is easy

- PEOU3: Overall, Moodle is easy to use

- PEOU4: Learning how to use Moodle is easy for me 
- PEOU5: I encounter minimal technical problems when using Moodle

Attitude Toward Using elearning (ATT)

ATT1-ATT4 | Multi-Point Scale: 1=strongly agree, 7=strongly disagree

- ATT1: I like the idea of using an elearning system

- ATT2: I think using an elearning system makes studying more interesting

- ATT3: Studying through an elearning system is a good idea

- ATT4: Overall, I like working with the elearning system

$\underline{\text { Behavioral Intention towards using elearning (BI) }}$

BI1-BI3 | Multi-Point Scale: 1=strongly agree, 7=strongly disagree

- BI1: I would want to use Moodle in my other classes

- BI2: If my other courses had an elearning system, I would use it

- BI3: If Moodle were not required for this course, I would still want to use it

\section{External Factors (only PU)}

$\underline{\mathrm{PU} \text { of labs (PU-LAB) }}$

- PU-LAB1-3 | Multi-Point Scale: 1=strongly agree, $7=$ strongly disagree

- PU-LAB1: Accessing the labs in Moodle makes it easier to complete them.

- PU-LAB2: I prefer to access the labs through the elearning system instead of through a USB drive.

- PU-LAB3: I like having access to the labs through Moodle.

$\underline{\mathrm{PU} \text { of video resources (PU-VR) }}$

PU-VR1-3 | Multi-Point Scale: 1=strongly agree, 7=strongly disagree

- PU-VR1: The lecture videos in Moodle help me understand the course topics.

- PU-VR2: The lecture videos in the elearning system make it easier to study the course content.

- PU-VR3: The lecture videos in Moodle improve my understanding of the ICT topics.

$\underline{\mathrm{PU} \text { of online presentations (PU-PR) }}$

PU-PR1-3 | Multi-Point Scale: 1=strongly agree, 7=strongly disagree 
- PU-PR1: The online presentations in Moodle help me understand the course topics.

- PU-PR2: The online presentations in the elearning system make it easier to study course content.

- PU-PR3: The online presentations in Moodle improve my understanding of the ICT topics.

$\underline{\text { PU of online grades (PU-OG) }}$

PU-OG1-3 | Multi-Point Scale: 1=strongly agree, 7=strongly disagree

- PU-OG1: The online grades in Moodle motivate me to complete my assignments.

- PU-OG2: The online grade in the elearning system help me track my coursework.

- PU-OG3: I find that the online grades in Moodle are a useful feature.

$\underline{\text { PU of announcements (PU-AN) }}$

PU-AN1-3 | Multi-Point Scale: 1=strongly agree, 7=strongly disagree

- PU-AN1: The announcements in Moodle keep me informed of class activities.

- PU-AN2: I read the announcements in the elearning system or in the email to know the upcoming assignments.

- PU-AN3: The announcements in Moodle keep me engaged in the course.

\section{Survey II - Russian}

\section{Демографические данные (DEM)}

- DEM1: Пол

○ Варианты ответа: М/Ж, предпочитаю не отвечать

- DEM2: Группа

○ Краткий ответ:

- DEM3: Мистер Хетрик - мой преподаватель

○ Варианты ответа: да/нет, предпочитаю не отвечать

- DEM4: Уровень владения английским языком

○ Варианты ответа: A1, A2, B1, B2, C1, C2, другой

\section{$\underline{\text { Технология (TEC) }}$}

- TEC1: Мой мобильный подсоединен к интернету

○ Многоуровневая шкала: 1 - всегда, 5 - никогда 
- ТЕС2: Я предпочитаю выходить в интернет через

○ Варианты ответа: Мобильное устройство, компьютер (ноутбук или ПК), оба варианта

- TEC3: У меня дома есть такие виды компьютера как

○ Возможны несколько вариантов: ПК, ноутбук, планшет, нет

- TEC4: Интернет соединение у меня дома это

○ Варианты ответа: оптоволокно, цифровая абонентская линия, соединение по телефонной линии, 3G/4G модем, другое, не знаю

- TEC5: Насколько быстрое, вы считаете, интернет соединение у вас дома

○ Многоуровневая шкала: $1=$ очень быстрое, $7=$ =чень медленное

- TEC6: По вашему мнению, насколько вам сложно или просто пользоваться интернетом?

○ Многоуровневая шкала: 1=очень просто, $7=$ очень сложно

- TEC7: По вашему мнению, насколько вам сложно или просто пользоваться компьютером (ноутбуком)?

○ Многоуровневая шкала: $1=$ очень просто, $7=$ очень сложно

- TEC8: По вашему мнению, насколько вам сложно или просто пользоваться смартфоном?

○ Многоуровневая шкала: $1=$ очень просто, $7=$ очень сложно

\section{Готовность технологии (TR)}

TR1-TR7 | Многоуровневая шкала: 1=полностью согласен 7=полностью не согласен

- TR1: Пользоваться браузером легко для меня

- TR2: Я с легкостью могу найти в Интернете то, что я хочу

- TR3: Мне легко пользоваться навигацией внутри веб-сайтов

- TR4: я могу легко поделиться с друзьями и семьей изображением (фото) с помощью социальных сетей, таких как Instagram, Facebook, OK.ru или $\mathrm{Vk}$

- TR5: я могу с лёгкостью сделать снимок и разместить его в социальных сетях

- TR6: я легко могу создавать новые учетные записи на сайтах

- TR7: пользоваться кнопками медиа плеера в веб-браузере или мобильном приложении очень легко для меня 
Готовность ученика (LR)

LR1-LR6 | Многоуровневая шкала: 1=полностью согласен 7=полностью не согласен

- LR1: я хотел бы учиться с помощью веб-технологий

- LR2: мне нравится идея обучения в режиме онлайн

- LR3: наличие онлайн ресурсов делает моё обучение более интересным

- LR4: я думаю, что веб-технологии могут улучшить мое обучение

- LR5: использование веб-технологий может помочь мне учиться

- LR6: для выполнения задания, я бы скорее использовал электронный ресурс (веб-сайт или электронный журнал) чем книгу/учебник

\section{Содержание готовности (CR)}

CR1-CR6 | Многоуровневая шкала: 1=полностью согласен 7=полностью не согласен

- CR1: я чувствую, что я готов учиться, используя Интернет

- CR2: я могу учиться одинаково хорошо - и слушая лекцию в аудитории, и просматривая онлайн видео дома

- CR3: я хотел бы обучаться и на лекции в аудитории, и при помощи презентации в Интернете

- CR4: я думаю, я мог бы узнать больше через онлайн ресурсы, чем слушая лекции в аудитории

- CR5: я думаю, что образование должно включать как обучение в классе, так и обучение онлайн.

- CR6: я бы предпочел, чтобы мой преподаватель давал мне задания, для выполнения которых требуется Интернет

Предыдущий опыт интернет обучения (PEX)

PEX1-PEX2 | Варианты ответа: да, нет, не знаю

PEX3-РЕX7 | Многоуровневая шкала: 1=всегда, 7=никогда

- PEX1: В старших классах моей школы ученики могли пользоваться компьютерным классом

- PЕХ2: В старших классах моей школы был образовательный портал

- РЕХЗ: Я пользовался образовательным порталом в старших классах 
- PEX4: Я сдавал домашние задания через образовательный портал в старших классах

- PEX5: Я получал доступ к учебным материалам через образовательный портал в старших классах

- PEX6: Мой учитель отправлял мне задания через образовательный портал в старших классах

- PEX7: Мой учитель общался с нами через образовательный портал в старших классах

Оцениваемая практичность интернет обучения (PU)

PU1-PU6 | Многоуровневая шкала: 1=полностью согласен 7=полностью не согласен

- PU1: Использование системы электронного обучения помогает мне изучать содержание учебного курса

- PU2: Использование системы электронного обучения помогает мне организовать мое обучение

- PU3: Использование системы электронного обучения облегчает изучение содержания учебного курса

- PU4: Использование системы электронного обучения полезно для моего обучения

Оцениваемая простота использования системы интернет обучения (PEOU)

PEOU1-PEOU6 | Многоуровневая шкала: 1=полностью согласен

$7=$ полностью не согласен

- PEOU1: Moodle сайт быстро загружается

- PEOU2: Навигация в системе электронного обучения проста

- PEOU3: В целом, сайтом Moodle легко пользоваться

- PEOU4: Мне просто научиться пользоваться сайтом Moodle

- PEOU5: Я почти не сталкиваюсь с техническими проблемами, когда пользуюсь сайтом Moodle

Отношение к использованию интернет обучения (АТT)

ATT1-АТT6 | Многоуровневая шкала: 1=полностью согласен 7=полностью не согласен 
- ATT1: Мне нравится идея использования системы электронного обучения

- ATT2: Я считаю, что система электронного обучения делает учебу более интересной

- ATT3: Учебный процесс через систему электронного обучения это хорошая идея

- ATT4: В целом мне нравиться работать с системой электронного обучения

Намерение использовать электронное обучение (BI)

BI1-BI6 | Многоуровневая шкала: 1=полностью согласен 7=полностью не согласен

- BI1: Я бы хотел(а) пользоваться Moodle на других моих занятиях

- ВI2: Если бы по другим дисциплинам была система электронного обучения, я бы ей пользовался (-лась)

- BI3: Если бы Moodle не был предложен для данного курса, я бы все равно хотел (а) им пользоваться.

Внешние факторы (только оцениваемая практичность электронного обучения PU)

Практическая значимость лабораторных занятий (PU-LAB)

PU-LAB1-3 Многоуровневая шкала: 1=полностью согласен, 7=совершенно не согласен

- PU-LAB1: Доступ к лабораторным занятиям в Moodle упрощает их выполнение

- PU-LAB2: Я предпочитаю получать доступ к лабораторным занятиям через электронную систему, чем через USB устройство

- PU-LAB3: Мне нравится иметь доступ к лабораторным занятиям через Moodle

Практическая значимость видео ресурсов (PU-VR)

PU-VR 1-3 | Многоуровневая шкала: 1=полностью согласен, 7=совершенно не согласен

- PU-VR1: Видео лекции в Moodle помогают мне понять темы курса 
- PU-VR2: Видео лекции в электронной системе упрощают изучение содержания курса

- PU-VR3: Видео лекции в Moodle улучшают понимание тем по ИКТ

Практическая значимость онлайн презентаций (PU-PR)

PU-PR1-3 | Многоуровневая шкала: 1=полностью согласен, 7=совершенно

не согласен

- PU-PR1: Онлайн презентации в Moodle помогают мне понять темы курса

- PU-PR2: Онлайн презентации в электронной системе упрощают изучение содержания курса

- PU-PR3: Онлайн презентации в Moodle улучшают понимание тем по ИКТ

Практическая значимость онлайн оценок (PU-OG)

PU-OG1-3 | Многоуровневая шкала: 1=полностью согласен, 7=совершенно не согласен

- PU-OG1: Онлайн оценки в Moodle мотивируют меня к выполнению заданий

- PU-OG2: Онлайн оценки в электронной системе обучения помогают мне отслеживать мою успеваемость по курсу

- PU-OG3: Я считаю, что онлайн оценки в Moodle являются полезным элементом

Практическая значимость объявлений (PU-AN)

PU-AN1-3 | Многоуровневая шкала: 1=полностью согласен, 7=совершенно не согласен

- PU-AN1: Объявления в Moodle обеспечивают мою информированность об учебной деятельности

- PU-AN2: Я читаю объявления в системе электронного обучения или по электронной почте, чтобы узнавать о предстоящих заданиях

- PU-AN3: Объявления в Moodle вовлекают меня в учебную деятельность 


\section{Survey III - English}

- Demographics (DEM)

○ DEM1: Gender

- Multiple Choice: M/F, Prefer not to answer

- DEM2: Group

○ Short Answer:

- DEM3: Mr. Hetrick is my instructor.

- Multiple Choice: Y/N, Prefer not to answer

- DEM4: Level of English

o Multiple Choice: A1, A2, B1, B2, C1, C2, other

\section{$\underline{\text { Technology (TEC) }}$}

- TEC1: My mobile phone is connected to the internet

○ Multi-Point Scale: 1=Always, $5=$ never

- TEC2: I prefer to browse the web using:

- Multiple Choice: A mobile device or, A computer (laptop or desktop), Both equally

- TEC3: I have the following computers at home

○ Multiple selection: desktop, laptop, tablet, none

- TEC4: My internet connection at home is

○ Multiple Choice: fiber optics, DSL, dial-up, 3G/4G modem, other, do not know, I do not have home internet at home

- TEC5: How fast do you consider your internet connection at home?

- Multi-Point Scale: $1=$ very fast, $7=$ very slow

- TEC6: In your opinion, how easy or difficult is for you to use the internet?

○ Multi-Point Scale: 1= 1=Extremely easy $2=$ Moderately easy $3=$ Slightly easy $4=$ Neither easy nor difficult $5=$ Slightly difficult 6=Moderately difficult $7=$ Extremely difficult

- TEC7: In your opinion, how easy or difficult is for you to use a computer (desktop or laptop)?

○ Multi-Point Scale: $1=$ Extremely easy $2=$ Moderately easy $3=$ Slightly easy $4=$ Neither easy nor difficult $5=$ Slightly difficult $6=$ Moderately difficult $7=$ Extremely difficult

- TEC8: In your opinion, how easy or difficult is for you to use a smartphone?

○ Multi-Point Scale: $1=$ Extremely easy $2=$ Moderately easy $3=$ Slightly easy $4=$ Neither easy nor difficult $5=$ Slightly difficult $6=$ Moderately difficult $7=$ Extremely difficult 


\section{$\underline{\text { Technology Readiness (TR) }}$}

TR1-TR7 | Multi-Point Scale: 1=strongly agree, 7=strongly disagree

- TR1: Using a web browser is easy for me

- TR2: I can find what I want on the internet with ease

- TR3: Navigating websites is easy for me

- TR4: It is easy for me to take a picture and post it on a social media site

- TR5: It is easy for me to create new accounts on websites

\section{Learner Readiness (LR)}

LR1-LR6 | Multi-Point Scale: 1=strongly agree, 7=strongly disagree

- LR1: I would like to study with the assistance of technology

- LR2: I like the idea of studying online

- LR3: I think technology can improve my learning

- LR4: Using technology can help me study

\section{Content Readiness (CR)}

CR1-CR6 | Multi-Point Scale: 1=strongly agree, 7=strongly disagree

- CR1: I feel prepared to study using the internet

- CR2: I could learn equally well by listening to a live lecture in a classroom or watching an online video at home

- CR3: I would want to learn both from classroom lectures and from online presentations

- CR4: I think education should include both learning in the classroom and learning online

- CR5: I would prefer if my instructor gave me assignments to do that required the internet

\section{Previous Elearning Experience (PEX)}

PEX1-PEX2 | Multiple Choice: yes, no, do not know

PEX3-PEX7 | Multi-Point Scale: 1=always, 7=never

- PEX1: My high school had a computer lab for the students to use:

- PEX2: My high school had an online learning portal

- PEX3: I used an online learning portal at my high school 
- PEX4: I submitted assignments through an online learning portal at my high school

- PEX5: I accessed course material through an online learning portal at my high school

- PEX6: My teacher posted assignments to an online learning portal at my high school

- PEX7: My high school teacher communicated with us through an online learning portal

Perceived Usefulness of elearning (PU)

PU1-PU4 | Multi-Point Scale: 1=strongly agree, 7=strongly disagree

- PU1: Using the elearning system helps me learn my course content

- PU2: Using the elearning system helps me organize my learning

- PU3: Using the elearning system makes it easier to study the course content

- PU4: Using the elearning system is useful to my learning

Perceived Ease of Use of elearning (PEOU)

PEOU1-PEOU3 | Multi-Point Scale: 1=strongly agree, 7=strongly disagree

- PEOU1: Navigating the elearning system is easy

- PEOU2: Overall, Moodle is easy to use

- PEOU3: Learning how to use Moodle is easy for me

$\underline{\text { Attitude Toward Using elearning (ATT) }}$

ATT1-ATT4 | Multi-Point Scale: 1=strongly agree, 7=strongly disagree

- ATT1: I like the idea of using an elearning system

- ATT2: I think using an elearning system makes studying more interesting

- ATT3: Studying through an elearning system is a good idea

- ATT4: Overall, I like working with the elearning system

Behavioral Intention towards using elearning (BI)

BI1-BI3 | Multi-Point Scale: 1=strongly agree, 7=strongly disagree

- BI1: I would want to use Moodle in my other classes

- BI2: If my other courses had an elearning system, I would use it

- BI3: If Moodle were not required for this course, I would still want to use it 
- BI4: I would like to use an elearning system in the future

\section{External factors (only PU)}

\section{$\underline{\mathrm{PU} \text { of labs (PU-LAB) }}$}

PU-LAB1-3 | Multi-Point Scale: 1=strongly agree, 7=strongly disagree

- PU-LAB1: Accessing the labs in Moodle makes it easier to complete them.

- PU-LAB2: I prefer to access the labs through the elearning system instead of through a USB drive.

- PU-LAB3: I like having access to the labs through Moodle.

$\underline{\text { PU of video resources (PU-VR) }}$

PU-VR1-3 | Multi-Point Scale: 1=strongly agree, 7=strongly disagree

- PU-VR1: The lecture videos in Moodle help me understand the course topics.

- PU-VR2: The lecture videos in the elearning system make it easier to study the course content.

- PU-VR3: The lecture videos in Moodle improve my understanding of the ICT topics.

\section{$\underline{\mathrm{PU} \text { of online presentations (PU-PR) }}$}

PU-PR1-3 | Multi-Point Scale: 1=strongly agree, 7=strongly disagree

- PU-PR1: The online presentations in Moodle help me understand the course topics.

- PU-PR2: The online presentations in the elearning system make it easier to study course content.

- PU-PR3: The online presentations in Moodle improve my understanding of the ICT topics.

$\underline{\text { PU of online grades (PU-OG) }}$

PU-OG1-3 | Multi-Point Scale: 1=strongly agree, 7=strongly disagree

- PU-OG1: The online grades in Moodle motivate me to complete my assignments.

- PU-OG2: The online grade in the elearning system help me track my coursework.

- PU-OG3: I find that the online grades in Moodle are a useful feature. 


\section{$\underline{\text { PU of announcements (PU-AN) }}$}

PU-AN1-3 | Multi-Point Scale: 1=strongly agree, 7=strongly disagree

- PU-AN1: The announcements in Moodle keep me informed of class activities.

- PU-AN2: I read the announcements in the elearning system or in the email to know the upcoming assignments.

- PU-AN3: The announcements in Moodle keep me engaged in the course.

\section{Survey III - Russian}

\section{Демографические данные (DEM)}

- DEM1: Пол

○ Варианты ответа: М/Ж, предпочитаю не отвечать

- DEM2: Группа

○ Краткий ответ:

- DEM3: Мистер Хетрик - мой преподаватель

○ Варианты ответа: да/нет, предпочитаю не отвечать

- DEM4: Уровень владения английским языком

○ Варианты ответа: A1, A2, B1, B2, C1, C2, другой

\section{Технология (ТЕС)}

- TEC1: Мой мобильный подсоединен к интернету

○ Многоуровневая шкала: 1 - всегда, 5 - никогда

- TEC2: Я предпочитаю выходить в интернет через

○ Варианты ответа: Мобильное устройство, компьютер (ноутбук или ПК), оба варианта

- $\quad$ TEC3: У меня дома есть такие виды компьютера как

○ Возможны несколько вариантов: ПК, ноутбук, планшет, нет

- TEC4: Интернет соединение у меня дома это

○ Варианты ответа: оптоволокно, цифровая абонентская линия, соединение по телефонной линии, 3G/4G модем, другое, не знаю

- TEC5: Насколько быстрое, вы считаете, интернет соединение у вас дома

○ Многоуровневая шкала: $1=$ очень быстрое, $7=$ очень медленное

- TEC6: По вашему мнению, насколько вам сложно или просто пользоваться интернетом?

○ Многоуровневая шкала: 1=очень просто 2=относительно просто $3=$ =остаточно просто 4= ни просто и ни сложно $5=$ =немного сложно 6=относительно сложно $7=$ очень сложно 
- TEC7: По вашему мнению, насколько вам сложно или просто пользоваться компьютером (ноутбуком)?

○ Многоуровневая шкала: $1=$ очень просто $2=$ относительно просто $3=$ достаточно просто 4= ни просто и ни сложно 5= немного сложно 6=относительно сложно 7= очень сложно

- TEC8: По вашему мнению, насколько вам сложно или просто пользоваться смартфоном?

○ Многоуровневая шкала: 1=очень просто 2=относительно просто $3=$ достаточно просто 4= ни просто и ни сложно 5= немного сложно 6=относительно сложно 7= очень сложно

Готовность технологии (TR)

TR1-TR7 | Многоуровневая шкала: 1=полностью согласен 7=полностью не согласен

- TR1: Пользоваться браузером легко для меня

- TR2: Я с легкостью могу найти в Интернете то, что я хочу

- TR3: Мне легко пользоваться навигацией внутри веб-сайтов

- TR4: я могу с лёгкостью сделать снимок и разместить его в социальных сетях

- TR5: я легко могу создавать новые учетные записи на сайтах

Готовность ученика (LR)

LR1-LR6 | Многоуровневая шкала: 1=пплностью согласен 7=полностью не согласен

- LR1: я хотел бы учиться с помощью веб-технологий

- LR2: мне нравится идея обучения в режиме онлайн

- LR3: я думаю, что веб-технологии могут улучшить мое обучение

- LR4: использование веб-технологий может помочь мне учиться

\section{$\underline{\text { Содержание готовности (CR) }}$}

CR1-CR6 | Многоуровневая шкала: 1=полностью согласен 7=полностью не согласен

- $\mathrm{CR} 1$ : я чувствую, что я готов учиться, используя Интернет 
- CR2: я могу учиться одинаково хорошо - и слушая лекцию в аудитории, и просматривая онлайн видео дома

- CR3: я хотел бы обучаться и на лекции в аудитории, и при помощи презентации в Интернете

- CR4: я думаю, что образование должно включать как обучение в классе, так и обучение онлайн.

- CR5: я бы предпочел, чтобы мой преподаватель давал мне задания, для выполнения которых требуется Интернет

\section{Предыдущий опыт интернет обучения (PEX)}

PEX1-PEX2 | Варианты ответа: да, нет, не знаю

PEX3-PEX7 | Многоуровневая шкала: 1=всегда, 7=никогда

- PEX1: В старших классах моей школы ученики могли пользоваться компьютерным классом

- PEX2: В старших классах моей школы был образовательный портал

- PЕХ3: Я пользовался образовательным порталом в старших классах

- $\mathrm{PEX4:} \mathrm{Я} \mathrm{сдавал} \mathrm{домашние} \mathrm{задания} \mathrm{через} \mathrm{образовательный} \mathrm{портал} \mathrm{в}$ старших классах

- $\mathrm{PEX5:} \mathrm{Я} \mathrm{получал} \mathrm{доступ} \mathrm{к} \mathrm{учебным} \mathrm{материалам} \mathrm{через} \mathrm{образовательный}$ портал в старших классах

- РЕХ6: Мой учитель отправлял мне задания через образовательный портал в старших классах

- PЕX7: Мой учитель общался с нами через образовательный портал в старших классах

Оцениваемая практичность интернет обучения (PU)

PU1-PU6 | Многоуровневая шкала: 1=полностью согласен 7=полностью не

согласен

- PU1: Использование системы электронного обучения помогает мне изучать содержание учебного курса

- PU2: Использование системы электронного обучения помогает мне организовать мое обучение

- PU3: Использование системы электронного обучения облегчает изучение содержания учебного курса

- PU4: Использование системы электронного обучения полезно для моего обучения 
Оцениваемая простота использования системы интернет обучения (PEOU)

PEOU1-PEOU6 | Многоуровневая шкала: 1=полностью согласен

7=полностью не согласен

- PEOU1: Навигация в системе электронного обучения проста

- PEOU2: В целом, сайтом Moodle легко пользоваться

- PEOU3: Мне просто научиться пользоваться сайтом Moodle

Отношение к использованию интернет обучения (АТT)

ATT1-ATT6 | Многоуровневая шкала: 1=полностью согласен 7=полностью не согласен

- ATT1: Мне нравится идея использования системы электронного обучения

- ATT2: Я считаю, что система электронного обучения делает учебу более интересной

- ATT3: Учебный процесс через систему электронного обучения это хорошая идея

- ATT4: В целом мне нравиться работать с системой электронного обучения

Намерение использовать электронное обучение (BI)

BI1-BI6 | Многоуровневая шкала: 1=полностью согласен 7=полностью не согласен

- BI1: Я бы хотел(а) пользоваться Moodle на других моих занятиях

- BI2: Если бы по другим дисциплинам была система электронного обучения, я бы ей пользовался (-лась)

- BI3: Если бы Moodle не был предложен для данного курса, я бы все равно хотел (а) им пользоваться.

Внешние факторы (только оцениваемая практичность электронного обучения PU)

Практическая значимость лабораторных занятий (PU-LAB)

PU-LAB1-3 Многоуровневая шкала: 1=полностью согласен, 7=совершенно не согласен 
- PU-LAB1: Доступ к лабораторным занятиям в Moodle упрощает их выполнение

- PU-LAB2: Я предпочитаю получать доступ к лабораторным занятиям через электронную систему, чем через USB устройство

- PU-LAB3: Мне нравится иметь доступ к лабораторным занятиям через Moodle

Практическая значимость видео ресурсов (PU-VR)

PU-VR1-3 | Многоуровневая шкала: 1=полностью согласен, 7=совершенно не согласен

- PU-VR1: Видео лекции в Moodle помогают мне понять темы курса

- PU-VR2: Видео лекции в электронной системе упрощают изучение содержания курса

- PU-VR3: Видео лекции в Moodle улучшают понимание тем по ИКТ

Практическая значимость онлайн презентаций (PU-PR)

PU-PR1-3 | Многоуровневая шкала: 1=полностью согласен, 7=совершенно не согласен

- PU-PR1: Онлайн презентации в Moodle помогают мне понять темы курса

- PU-PR2: Онлайн презентации в электронной системе упрощают изучение содержания курса

- PU-PR3: Онлайн презентации в Moodle улучшают понимание тем по ИКТ

Практическая значимость онлайн оценок (PU-OG)

PU-OG1-3 | Многоуровневая шкала: 1=полностью согласен, 7=совершенно не согласен

- PU-OG1: Онлайн оценки в Moodle мотивируют меня к выполнению заданий

- PU-OG2: Онлайн оценки в электронной системе обучения помогают мне отслеживать мою успеваемость по курсу

- PU-OG3: Я считаю, что онлайн оценки в Moodle являются полезным элементом 
Практическая значимость объявлений (PU-AN)

PU-AN1-3 | Многоуровневая шкала: 1=полностью согласен, 7=совершенно не согласен

- PU-AN1: Объявления в Moodle обеспечивают мою информированность об учебной деятельности

- PU-AN2: Я читаю объявления в системе электронного обучения или по электронной почте, чтобы узнавать о предстоящих заданиях

- PU-AN3: Объявления в Moodle вовлекают меня в учебную деятельность 
APPENDIX B

PCA Analysis Tables: Survey I 


\section{Original TAM: Survey I PCA}

\section{Table B1: Survey I, Original TAM PCA: Correlation Matrix ${ }^{\text {a }}$}

\begin{tabular}{|c|c|c|c|c|c|c|c|c|c|c|c|c|c|c|c|}
\hline & & & & & PEO & PEO & PEO & PEO & ATT & ATT & ATT & & & & \\
\hline & PU1 & PU3 & PU5 & PU6 & U1 & $\mathrm{U} 2$ & U5 & U6 & & & 6 & BI2 & BI3 & BI4 & BI5 \\
\hline PU1 & 1.000 & & & & & & & & & & & & & & \\
\hline PU3 & .677 & 1.000 & & & & & & & & & & & & & \\
\hline PU5 & .728 & .778 & 1.000 & & & & & & & & & & & & \\
\hline PU6 & .569 & .731 & .620 & 1.000 & & & & & & & & & & & \\
\hline PEOU1 & .474 & .459 & .560 & .557 & 1.000 & & & & & & & & & & \\
\hline PEOU2 & .440 & .482 & .508 & .479 & .597 & 1.000 & & & & & & & & & \\
\hline PEOU5 & .556 & .662 & .594 & .518 & .676 & .766 & 1.000 & & & & & & & & \\
\hline PEOU6 & .567 & .643 & .533 & .551 & .651 & .684 & .787 & 1.000 & & & & & & & \\
\hline ATT4 & .724 & .688 & .673 & .653 & .573 & .475 & .558 & .648 & 1.000 & & & & & & \\
\hline ATT5 & .532 & .446 & .459 & .437 & .496 & .358 & .419 & .446 & .620 & 1.000 & & & & & \\
\hline ATT6 & .692 & .606 & .652 & .547 & .503 & .546 & .546 & .586 & .728 & .649 & 1.000 & & & & \\
\hline BI2 & .602 & .625 & .723 & .565 & .627 & .436 & .596 & .560 & .712 & .609 & .643 & 1.000 & & & \\
\hline BI3 & .479 & .494 & .659 & .503 & .453 & .311 & .343 & .358 & .559 & .519 & .429 & .683 & 1.000 & & \\
\hline BI4 & .591 & .579 & .576 & .633 & .520 & .482 & .490 & .559 & .715 & .509 & .620 & .748 & .517 & 1.000 & \\
\hline BI5 & .398 & .374 & .499 & .392 & .339 & .220 & .249 & .308 & .625 & .341 & .447 & .633 & .676 & .558 & 1.000 \\
\hline
\end{tabular}

a. Determinant $=1.477 \mathrm{E}-6$ 
Table B2: Survey I, Original TAM PCA: Anti-image Correlation

\begin{tabular}{|c|c|c|c|c|c|c|c|c|c|c|c|c|c|c|c|}
\hline & & & & & PEO & PEO & PEO & PEO & ATT & ATT & ATT & & & & \\
\hline & PU1 & PU3 & PU5 & PU6 & $\mathrm{U} 1$ & $\mathrm{U} 2$ & U5 & U6 & 4 & 5 & 6 & BI2 & BI3 & BI4 & BI5 \\
\hline PU1 & $.936^{\mathrm{a}}$ & & & & & & & & & & & & & & \\
\hline PU3 & .021 & $.856^{\mathrm{a}}$ & & & & & & & & & & & & & \\
\hline PU5 & -.340 & -.472 & $.871^{\mathrm{a}}$ & & & & & & & & & & & & \\
\hline PU6 & -.005 & -.473 & .102 & $.902^{\mathrm{a}}$ & & & & & & & & & & & \\
\hline PEOU1 & .085 & .388 & -.219 & -.311 & $.893^{\mathrm{a}}$ & & & & & & & & & & \\
\hline PEOU2 & .161 & .230 & -.189 & -.098 & -.047 & $.844^{\mathrm{a}}$ & & & & & & & & & \\
\hline PEOU5 & -.138 & -.333 & .074 & .131 & -.246 & -.531 & $.857^{\mathrm{a}}$ & & & & & & & & \\
\hline PEOU6 & -.056 & -.219 & .221 & .076 & -.223 & -.160 & -.315 & $.932^{\mathrm{a}}$ & & & & & & & \\
\hline ATT4 & -.253 & -.152 & .008 & -.100 & -.104 & .043 & .069 & -.193 & $.931^{\mathrm{a}}$ & & & & & & \\
\hline ATT5 & -.070 & -.027 & .273 & .064 & -.138 & .034 & .010 & .073 & -.232 & $.854^{\mathrm{a}}$ & & & & & \\
\hline ATT6 & -.198 & .016 & -.197 & -.032 & .099 & -.251 & .114 & -.080 & -.131 & -.392 & $.915^{\mathrm{a}}$ & & & & \\
\hline BI2 & .125 & .005 & -.242 & .117 & -.178 & .296 & -.311 & .042 & .025 & -.195 & -.101 & $.897^{a}$ & & & \\
\hline $\mathrm{BI} 3$ & -.023 & .021 & -.331 & -.152 & -.002 & -.106 & .148 & -.045 & .130 & -.371 & .286 & -.181 & $.845^{\mathrm{a}}$ & & \\
\hline BI4 & -.134 & -.015 & .142 & -.244 & .050 & -.238 & .195 & -.076 & -.137 & .035 & -.013 & -.462 & .092 & $.913^{\mathrm{a}}$ & \\
\hline BI5 & .117 & .111 & .042 & .062 & .072 & .044 & .030 & .039 & -.414 & .311 & -.136 & -.202 & -.474 & -.101 & $.827^{a}$ \\
\hline
\end{tabular}

a. Measures of Sampling Adequacy (MSA)

Table B3: Survey I, Original TAM PCA: KMO and Bartlett's Test

Kaiser-Meyer-Olkin Measure of Sampling Adequacy.

.888

Bartlett's Test of

Approx. Chi-Square

592.954

Sphericity

df

105

Sig.

.000 
Table B4: Survey I, Original TAM PCA: Communalities

\begin{tabular}{lrr}
\hline & \multicolumn{1}{c}{ Initial } & Extraction \\
\hline PU1 & 1.000 & .780 \\
PU3 & 1.000 & .875 \\
PU5 & 1.000 & .796 \\
PU6 & 1.000 & .681 \\
PEOU1 & 1.000 & .754 \\
PEOU2 & 1.000 & .781 \\
PEOU5 & 1.000 & .861 \\
PEOU6 & 1.000 & .784 \\
ATT4 & 1.000 & .799 \\
ATT5 & 1.000 & .850 \\
ATT6 & 1.000 & .797 \\
BI2 & 1.000 & .810 \\
BI3 & 1.000 & .781 \\
BI4 & 1.000 & .659 \\
BI5 & 1.000 & .811 \\
\hline
\end{tabular}

Extraction Method: Principal

Component Analysis. 
Table B5: $\quad$ Survey I, Original TAM PCA: Total Variance Explained

\begin{tabular}{|c|c|c|c|c|c|c|c|}
\hline \multirow[b]{2}{*}{$\begin{array}{l}\text { Comp } \\
\text { onent }\end{array}$} & \multicolumn{3}{|c|}{ Initial Eigenvalues } & \multicolumn{3}{|c|}{$\begin{array}{c}\text { Extraction Sums of Squared } \\
\text { Loadings }\end{array}$} & \multirow{2}{*}{$\begin{array}{c}\text { Rotation } \\
\text { Sums of } \\
\text { Squared } \\
\text { Loadings a } \\
\text { Total } \\
\end{array}$} \\
\hline & Total & $\begin{array}{c}\% \text { of } \\
\text { Variance }\end{array}$ & $\begin{array}{c}\text { Cumulative } \\
\%\end{array}$ & Total & $\begin{array}{c}\% \text { of } \\
\text { Variance }\end{array}$ & $\begin{array}{c}\text { Cumulative } \\
\% \\
\end{array}$ & \\
\hline 1 & 8.832 & 58.880 & 58.880 & 8.832 & 58.880 & 58.880 & 6.215 \\
\hline 2 & 1.451 & 9.673 & 68.553 & 1.451 & 9.673 & 68.553 & 5.249 \\
\hline 3 & .789 & 5.257 & 73.810 & .789 & 5.257 & 73.810 & 6.271 \\
\hline 4 & .747 & 4.979 & 78.789 & .747 & 4.979 & 78.789 & 5.353 \\
\hline 5 & .564 & 3.763 & 82.552 & & & & \\
\hline 6 & .518 & 3.455 & 86.007 & & & & \\
\hline 7 & .388 & 2.588 & 88.595 & & & & \\
\hline 8 & .365 & 2.430 & 91.025 & & & & \\
\hline 9 & .350 & 2.334 & 93.360 & & & & \\
\hline 10 & .282 & 1.877 & 95.236 & & & & \\
\hline 11 & .204 & 1.357 & 96.594 & & & & \\
\hline 12 & .177 & 1.177 & 97.771 & & & & \\
\hline 13 & .131 & .871 & 98.642 & & & & \\
\hline 14 & .115 & .764 & 99.406 & & & & \\
\hline 15 & .089 & .594 & 100.000 & & & & \\
\hline
\end{tabular}

Extraction Method: Principal Component Analysis.

a. When components are correlated, sums of squared loadings cannot be added to obtain a total variance. 
Table B6: Survey I, Original TAM PCA: Structure Matrix

\begin{tabular}{lcccc}
\hline & \multicolumn{4}{c}{ Component } \\
& 1 & 2 & 3 & 4 \\
\hline PU3 & .929 & & & \\
PU5 & .835 & & & \\
PU1 & .816 & & & .685 \\
PU6 & .793 & & & \\
BI5 & & .898 & & \\
BI3 & & .880 & & \\
BI2 & & .806 & & \\
BI4 & & .678 & & \\
PEOU5 & & & .917 & \\
PEOU2 & & & .880 & \\
PEOU6 & & & .869 & \\
PEOU1 & & & .831 & \\
ATT5 & & & & .917 \\
ATT6 & & & & .838 \\
ATT4 & .727 & & & .750 \\
\hline
\end{tabular}

Extraction Method: Principal Component Analysis.

Rotation Method: Oblimin with Kaiser Normalization.

Suppressed coefficients $<.65$ for readability.

Table B7: $\quad$ Survey I, Original TAM PCA: Component Correlation Matrix

\begin{tabular}{lrrrr}
\hline Component & \multicolumn{1}{c}{1} & \multicolumn{1}{c}{2} & \multicolumn{1}{c}{3} & \multicolumn{1}{c}{4} \\
\hline 1 & 1.000 & & & \\
2 & .455 & 1.000 & & \\
3 & .566 & .390 & 1.000 & \\
4 & .478 & .484 & .498 & 1.000 \\
\hline
\end{tabular}

Extraction Method: Principal Component Analysis.

Rotation Method: Oblimin with Kaiser

Normalization. 
Simplified TAM: Survey I PCA

Table B8: $\quad$ Survey I, Simplified TAM PCA: Correlation Matrix ${ }^{\text {a }}$

\begin{tabular}{llllllllllllllll}
\hline & & & & & \multicolumn{1}{c}{ PEO } & PEO & PEO & PEO & PEO & & & & \\
& PU1 & PU2 & PU3 & PU5 & PU6 & U1 & U2 & U4 & U5 & U6 & BI2 & BI3 & BI4 & BI5 \\
\hline PU1 & 1.000 & & & & & & & & & & & & & \\
PU2 & .663 & 1.000 & & & & & & & & & & & & \\
PU3 & .677 & .693 & 1.000 & & & & & & & & & & & \\
PU5 & .728 & .662 & .778 & 1.000 & & & & & & & & & & \\
PU6 & .569 & .665 & .731 & .620 & 1.000 & & & & & & & & & \\
PEOU1 & .474 & .595 & .459 & .560 & .557 & 1.000 & & & & & & & & \\
PEOU2 & .440 & .448 & .482 & .508 & .479 & .597 & 1.000 & & & & & & & \\
PEOU4 & .547 & .475 & .448 & .494 & .425 & .501 & .549 & 1.000 & & & & & & \\
PEOU5 & .556 & .539 & .662 & .594 & .518 & .676 & .766 & .560 & 1.000 & & & & & \\
PEOU6 & .567 & .534 & .643 & .533 & .551 & .651 & .684 & .465 & .787 & 1.000 & & & & \\
BI2 & .602 & .681 & .625 & .723 & .565 & .627 & .436 & .502 & .596 & .560 & 1.000 & & & \\
BI3 & .479 & .604 & .494 & .659 & .503 & .453 & .311 & .355 & .343 & .358 & .683 & 1.000 & & \\
BI4 & .591 & .585 & .579 & .576 & .633 & .520 & .482 & .511 & .490 & .559 & .748 & .517 & 1.000 & \\
BI5 & .398 & .464 & .374 & .499 & .392 & .339 & .220 & .219 & .249 & .308 & .633 & .676 & .558 & 1.000 \\
\hline
\end{tabular}

a. Determinant $=7.861 \mathrm{E}-6$

Table B9: Survey I, Simplified TAM PCA: KMO and Bartlett's Test

\begin{tabular}{|c|c|c|}
\hline \multicolumn{2}{|c|}{$\begin{array}{l}\text { Kaiser-Meyer-Olkin Measure of Sampling } \\
\text { Adequacy. }\end{array}$} & .886 \\
\hline Bartlett's Test of & Approx. Chi-Square & 523.038 \\
\hline Sphericity & $\mathrm{df}$ & 91 \\
\hline & Sig. & .000 \\
\hline
\end{tabular}


Table B10: Survey I, Simplified TAM PCA: Communalities

\begin{tabular}{lrr}
\hline & Initial & Extraction \\
\hline PU1 & 1.000 & .720 \\
PU2 & 1.000 & .716 \\
PU3 & 1.000 & .860 \\
PU5 & 1.000 & .776 \\
PU6 & 1.000 & .679 \\
PEOU1 & 1.000 & .709 \\
PEOU2 & 1.000 & .783 \\
PEOU4 & 1.000 & .507 \\
PEOU5 & 1.000 & .841 \\
PEOU6 & 1.000 & .748 \\
BI2 & 1.000 & .812 \\
BI3 & 1.000 & .764 \\
BI4 & 1.000 & .656 \\
BI5 & 1.000 & .819 \\
\hline Extaction
\end{tabular}

Extraction Method: Principal

Component Analysis. 
Table B11: Survey I, Simplified TAM PCA: Total Variance Explained

\begin{tabular}{|c|c|c|c|c|c|c|c|}
\hline \multirow[b]{2}{*}{$\begin{array}{l}\text { Compo } \\
\text { nent }\end{array}$} & \multicolumn{3}{|c|}{ Initial Eigenvalues } & \multicolumn{3}{|c|}{$\begin{array}{c}\text { Extraction Sums of Squared } \\
\text { Loadings }\end{array}$} & \multirow{2}{*}{$\begin{array}{c}\text { Rotation } \\
\text { Sums of } \\
\text { Squared } \\
\text { Loadings a } \\
\\
\text { Total }\end{array}$} \\
\hline & Total & $\begin{array}{c}\% \text { of } \\
\text { Variance }\end{array}$ & $\begin{array}{c}\text { Cumulative } \\
\%\end{array}$ & Total & $\begin{array}{c}\% \text { of } \\
\text { Variance }\end{array}$ & $\begin{array}{c}\text { Cumulative } \\
\%\end{array}$ & \\
\hline 1 & 8.125 & 58.039 & 58.039 & 8.125 & 58.039 & 58.039 & 6.945 \\
\hline 2 & 1.474 & 10.529 & 68.568 & 1.474 & 10.529 & 68.568 & 4.692 \\
\hline 3 & .790 & 5.643 & 74.211 & .790 & 5.643 & 74.211 & 6.305 \\
\hline 4 & .645 & 4.611 & 78.822 & & & & \\
\hline 5 & .529 & 3.779 & 82.601 & & & & \\
\hline 6 & .500 & 3.570 & 86.171 & & & & \\
\hline 7 & .414 & 2.954 & 89.125 & & & & \\
\hline 8 & .330 & 2.359 & 91.484 & & & & \\
\hline 9 & .296 & 2.116 & 93.599 & & & & \\
\hline 10 & .272 & 1.946 & 95.545 & & & & \\
\hline 11 & .245 & 1.751 & 97.296 & & & & \\
\hline 12 & .167 & 1.192 & 98.488 & & & & \\
\hline 13 & .126 & .900 & 99.388 & & & & \\
\hline 14 & .086 & .612 & 100.000 & & & & \\
\hline
\end{tabular}

Extraction Method: Principal Component Analysis.

a. When components are correlated, sums of squared loadings cannot be added to obtain a total variance. 
Table B12: Survey I, Simplified TAM PCA: Structure Matrix

\begin{tabular}{lccc}
\hline \multicolumn{4}{c}{ Component } \\
& 1 & 2 & 3 \\
\hline PU3 & .923 & & \\
PU5 & .861 & & \\
PU1 & .848 & & \\
PU2 & .826 & & \\
PU6 & .822 & & \\
BI5 & & .902 & \\
BI3 & & .860 & \\
BI2 & .717 & .814 & \\
BI4 & .679 & .693 & \\
PEOU5 & .662 & & .909 \\
PEOU2 & & & .878 \\
PEOU6 & .656 & & .857 \\
PEOU1 & & & .815 \\
PEOU4 & & & .702 \\
\hline
\end{tabular}

Extraction Method: Principal

Component Analysis.

Rotation Method: Oblimin with Kaiser

Normalization.

Suppressed coefficients $<.65$ for readability.

Table B13: Survey I, Simplified TAM PCA: Component Correlation Matrix

\begin{tabular}{lrrr}
\hline Component & \multicolumn{1}{c}{1} & \multicolumn{1}{c}{2} & 3 \\
\hline 1 & 1.000 & & \\
2 & .642 & 1.000 & \\
3 & .593 & .363 & 1.000 \\
\hline
\end{tabular}

Extraction Method: Principal Component

Analysis.

Rotation Method: Oblimin with Kaiser

Normalization. 
APPENDIX C

PCA Analysis Tables: Survey II 
Original TAM: Survey II PCA

Table C1: Survey II, Original TAM PCA: Correlation Matrix ${ }^{\text {a }}$

\begin{tabular}{lrrrrrrrr}
\hline & \multicolumn{1}{c}{ PU3 } & PU4 & PEOU1 & PEOU5 & ATT1 & ATT2 & BI1 & BI2 \\
\hline PU3 & 1.000 & & & & & & & \\
PU4 & .794 & 1.000 & & & & & & \\
PEOU1 & .409 & .380 & 1.000 & & & & & \\
PEOU5 & .313 & .245 & .483 & 1.000 & & & & \\
ATT1 & .602 & .528 & .406 & .236 & 1.000 & & & \\
ATT2 & .461 & .458 & .338 & .124 & .589 & 1.000 & & \\
BI1 & .444 & .270 & .187 & .016 & .445 & .383 & 1.000 & \\
BI2 & .430 & .286 & .283 & .154 & .480 & .391 & .682 & 1.000 \\
\hline
\end{tabular}

a. Determinant $=.028$

Table C2: Survey II, Original TAM PCA: Anti-image Matrices

\begin{tabular}{lcccccccc}
\hline & PU3 & PU4 & PEOU1 & PEOU5 & ATT1 & ATT2 & BI1 & BI2 \\
\hline PU3 & $.752^{\mathrm{a}}$ & & & & & & & \\
PU4 & -.695 & $.730^{\mathrm{a}}$ & & & & & & \\
PEOU1 & -.027 & -.081 & $.821^{\mathrm{a}}$ & & & & & \\
PEOU5 & -.174 & .063 & -.405 & $.666^{\mathrm{a}}$ & & & & \\
ATT1 & -.192 & -.067 & -.117 & -.034 & $.887^{\mathrm{a}}$ & & & \\
ATT2 & .029 & -.160 & -.114 & .080 & -.354 & $.867^{\mathrm{a}}$ & & \\
BI1 & -.239 & .144 & .027 & .175 & -.075 & -.091 & $.716^{\mathrm{a}}$ & \\
BI2 & -.050 & .053 & -.073 & -.082 & -.144 & -.062 & -.564 & $.772^{\mathrm{a}}$ \\
\hline
\end{tabular}

a. Measures of Sampling Adequacy (MSA) 
Table C3: Survey II, Original TAM PCA: KMO and Bartlett's Test

\begin{tabular}{llr}
\hline Kaiser-Meyer-Olkin Measure of Sampling Adequacy. & .780 \\
Bartlett's Test of & Approx. Chi-Square & 270.298 \\
Sphericity & df & 28 \\
& Sig. & .000 \\
\hline
\end{tabular}

Table C4: Survey II, Original TAM PCA: Communalities

\begin{tabular}{lcr}
\hline & Initial & Extraction \\
\hline PU3 & 1.000 & .903 \\
PU4 & 1.000 & .908 \\
PEOU1 & 1.000 & .743 \\
PEOU5 & 1.000 & .832 \\
ATT1 & 1.000 & .717 \\
ATT2 & 1.000 & .874 \\
BI1 & 1.000 & .850 \\
BI2 & 1.000 & .843 \\
\hline
\end{tabular}

Extraction Method: Principal

Component Analysis. 
Table C5: Survey II, Original TAM PCA: Total Variance Explained

\begin{tabular}{|c|c|c|c|c|c|c|c|}
\hline \multirow[b]{2}{*}{$\begin{array}{l}\text { Compo } \\
\text { nent }\end{array}$} & \multicolumn{3}{|c|}{ Initial Eigenvalues } & \multicolumn{3}{|c|}{$\begin{array}{c}\text { Extraction Sums of Squared } \\
\text { Loadings }\end{array}$} & \multirow{2}{*}{$\begin{array}{c}\text { Rotation } \\
\text { Sums of } \\
\text { Squared } \\
\text { Loadings a } \\
\\
\text { Total }\end{array}$} \\
\hline & Total & $\begin{array}{c}\% \text { of } \\
\text { Variance }\end{array}$ & $\begin{array}{c}\text { Cumulative } \\
\%\end{array}$ & Total & $\begin{array}{c}\% \text { of } \\
\text { Variance }\end{array}$ & $\begin{array}{c}\text { Cumulative } \\
\% \\
\end{array}$ & \\
\hline 1 & 3.806 & 47.576 & 47.576 & 3.806 & 47.576 & 47.576 & 2.851 \\
\hline 2 & 1.302 & 16.279 & 63.855 & 1.302 & 16.279 & 63.855 & 1.915 \\
\hline 3 & .904 & 11.303 & 75.158 & .904 & 11.303 & 75.158 & 2.504 \\
\hline 4 & .659 & 8.234 & 83.391 & 659 & 8.234 & 83.391 & 2.391 \\
\hline 5 & .470 & 5.879 & 89.270 & & & & \\
\hline 6 & .380 & 4.752 & 94.022 & & & & \\
\hline 7 & .305 & 3.816 & 97.837 & & & & \\
\hline 8 & .173 & 2.163 & 100.000 & & & & \\
\hline
\end{tabular}

Extraction Method: Principal Component Analysis.

a. When components are correlated, sums of squared loadings cannot be added to obtain a total variance.

Table C6: $\quad$ Survey II, Original TAM PCA: Structure Matrix

\begin{tabular}{lcccc}
\hline & \multicolumn{4}{c}{ Component } \\
& 1 & 2 & 3 & 4 \\
\hline PU4 & .947 & & & \\
PU3 & .938 & & & \\
PEOU5 & & .897 & & \\
PEOU1 & & .811 & & \\
BI1 & & & .916 & \\
BI2 & & & .912 & \\
ATT2 & & & & .934 \\
ATT1 & & & & .767 \\
\hline
\end{tabular}

Extraction Method: Principal Component Analysis.

Rotation Method: Oblimin with Kaiser

Normalization.

Suppressed coefficients $<.65$ for readability 
Table C7: Survey II, Original TAM PCA: Component Correlation Matrix

\begin{tabular}{lrrrr}
\hline Component & \multicolumn{1}{l}{1} & 2 & 3 & \multicolumn{1}{l}{4} \\
\hline 1 & 1.000 & & & \\
2 & .341 & 1.000 & & \\
3 & .383 & .165 & 1.000 & \\
4 & .466 & .223 & .409 & 1.000 \\
\hline
\end{tabular}

Extraction Method: Principal Component Analysis.

Rotation Method: Oblimin with Kaiser Normalization.

Simplified TAM: Survey II PCA

Table C8: $\quad$ Survey II, Simplified TAM PCA: Correlation Matrix a

\begin{tabular}{lrrrrrrrr}
\hline & \multicolumn{1}{c}{ PU1 } & \multicolumn{1}{c}{ PU3 } & PU4 & PEOU1 & PEOU4 & PEOU5 & BI1 & BI2 \\
\hline PU1 & 1.000 & & & & & & & \\
PU3 & .570 & 1.000 & & & & & & \\
PU4 & .626 & .794 & 1.000 & & & & & \\
PEOU1 & .264 & .409 & .380 & 1.000 & & & & \\
PEOU4 & .425 & .205 & .208 & .506 & 1.000 & & & \\
PEOU5 & .266 & .313 & .245 & .483 & .515 & 1.000 & & \\
BI1 & .273 & .444 & .270 & .187 & .140 & .016 & 1.000 & \\
BI2 & .307 & .430 & .286 & .283 & .193 & .154 & .682 & 1.000 \\
\hline
\end{tabular}

a. Determinant $=.026$

Table C9: $\quad$ Survey II, Simplified TAM PCA: KMO and Bartlett's Test

\begin{tabular}{llr}
\hline Kaiser-Meyer-Olkin Measure of Sampling Adequacy. & .711 \\
Bartlett's Test of & Approx. Chi-Square & 274.839 \\
Sphericity & df & 28 \\
& Sig. & .000 \\
\hline
\end{tabular}


Table C10: Survey II, Simplified TAM PCA: Communalities

\begin{tabular}{lcr}
\hline & Initial & Extraction \\
\hline PU1 & 1.000 & .657 \\
PU3 & 1.000 & .831 \\
PU4 & 1.000 & .880 \\
PEOU1 & 1.000 & .631 \\
PEOU4 & 1.000 & .715 \\
PEOU5 & 1.000 & .686 \\
BI1 & 1.000 & .851 \\
B12 & 1.000 & .832 \\
\hline
\end{tabular}

Extraction Method: Principal

Component Analysis.

Table C11: Survey II, Simplified TAM PCA: Total Variance Explained

\begin{tabular}{|c|c|c|c|c|c|c|c|}
\hline \multirow[b]{2}{*}{$\begin{array}{l}\text { Compo } \\
\text { nent }\end{array}$} & \multicolumn{3}{|c|}{ Initial Eigenvalues } & \multicolumn{3}{|c|}{$\begin{array}{c}\text { Extraction Sums of Squared } \\
\text { Loadings }\end{array}$} & \multirow{2}{*}{$\begin{array}{c}\text { Rotation } \\
\text { Sums of } \\
\text { Squared } \\
\text { Loadings } \\
\\
\text { Total }\end{array}$} \\
\hline & Total & $\begin{array}{c}\% \text { of } \\
\text { Variance }\end{array}$ & $\begin{array}{c}\text { Cumulative } \\
\% \\
\end{array}$ & Total & $\begin{array}{c}\% \text { of } \\
\text { Variance }\end{array}$ & $\begin{array}{c}\text { Cumulative } \\
\%\end{array}$ & \\
\hline$\overline{1}$ & 3.526 & 44.075 & 44.075 & 3.526 & 44.075 & 44.075 & 2.929 \\
\hline 2 & 1.483 & 18.537 & 62.612 & 1.483 & 18.537 & 62.612 & 2.451 \\
\hline 3 & 1.074 & 13.431 & 76.043 & 1.074 & 13.431 & 76.043 & 2.181 \\
\hline 4 & .666 & 8.330 & 84.372 & & & & \\
\hline 5 & .501 & 6.267 & 90.640 & & & & \\
\hline 6 & .327 & 4.092 & 94.732 & & & & \\
\hline 7 & .252 & 3.148 & 97.881 & & & & \\
\hline 8 & .170 & 2.119 & 100.000 & & & & \\
\hline
\end{tabular}

Extraction Method: Principal Component Analysis.

a. When components are correlated, sums of squared loadings cannot be added to obtain a total variance. 
Table C12: $\quad$ Survey II, Simplified TAM PCA: Structure Matrix

\begin{tabular}{lccc}
\hline & \multicolumn{3}{c}{ Component } \\
& 1 & 2 & 3 \\
\hline PU4 & .934 & & \\
PU3 & .898 & & \\
PU1 & .804 & & \\
PEOU4 & & .844 & \\
PEOU5 & & .822 & \\
PEOU1 & & .779 & \\
BI1 & & & .920 \\
BI2 & & & .908 \\
\hline
\end{tabular}

Extraction Method: Principal

Component Analysis.

Rotation Method: Oblimin with Kaiser

Normalization.

Suppressed coefficients $<.65$ for

readability.

Table C13: Survey II, Simplified TAM PCA: Component Correlation Matrix

\begin{tabular}{lrrr}
\hline Component & \multicolumn{1}{c}{1} & \multicolumn{1}{l}{2} & \multicolumn{1}{l}{3} \\
\hline 1 & 1.000 & & \\
2 & .389 & 1.000 & \\
3 & .379 & .187 & 1.000 \\
\hline
\end{tabular}

Extraction Method: Principal Component

Analysis.

Rotation Method: Oblimin with Kaiser

Normalization. 
APPENDIX D

PCA Analysis Tables: Survey III 
Original TAM: Survey III PCA

Table D1: Survey III, Original TAM PCA: Correlation Matrix ${ }^{\text {a }}$

\begin{tabular}{|c|c|c|c|c|c|c|c|c|c|c|c|}
\hline & PU1 & PU2 & PU3 & $\begin{array}{c}\text { PEOU } \\
1 \\
\end{array}$ & $\begin{array}{c}\text { PEOU } \\
2 \\
\end{array}$ & $\begin{array}{c}\text { PEOU } \\
3 \\
\end{array}$ & ATT2 & ATT3 & $\mathrm{BI} 2$ & $\mathrm{BI} 3$ & BI4 \\
\hline PU1 & 1.000 & & & & & & & & & & \\
\hline PU2 & .738 & 1.000 & & & & & & & & & \\
\hline PU3 & .590 & .659 & 1.000 & & & & & & & & \\
\hline PEOU1 & .441 & .375 & .563 & 1.000 & & & & & & & \\
\hline PEOU2 & .211 & .294 & .269 & .518 & 1.000 & & & & & & \\
\hline PEOU3 & .495 & .530 & .495 & .561 & .433 & 1.000 & & & & & \\
\hline ATT2 & .360 & .456 & .276 & .368 & .549 & .371 & 1.000 & & & & \\
\hline ATT3 & .626 & .546 & .522 & .611 & .592 & .586 & .743 & 1.000 & & & \\
\hline BI2 & .633 & .600 & .580 & .506 & .364 & .627 & .549 & .721 & 1.000 & & \\
\hline $\mathrm{BI} 3$ & .335 & .208 & .300 & .471 & .222 & .324 & .410 & .455 & .537 & 1.000 & \\
\hline BI4 & .423 & .441 & .442 & .356 & .190 & .520 & .301 & .323 & .636 & .479 & 1.000 \\
\hline
\end{tabular}

a. Determinant $=.001$ 
Table D2: Survey III, Original TAM PCA: Anti-image Correlation

\begin{tabular}{|c|c|c|c|c|c|c|c|c|c|c|c|}
\hline & PU1 & PU2 & PU3 & $\begin{array}{c}\text { PEOU } \\
1 \\
\end{array}$ & $\begin{array}{c}\text { PEOU } \\
2 \\
\end{array}$ & $\begin{array}{c}\text { PEOU } \\
3 \\
\end{array}$ & ATT2 & ATT3 & BI2 & BI3 & BI4 \\
\hline PU1 & $.805^{\mathrm{a}}$ & & & & & & & & & & \\
\hline PU2 & -.550 & $.774^{\mathrm{a}}$ & & & & & & & & & \\
\hline PU3 & .042 & -.422 & $.853^{\mathrm{a}}$ & & & & & & & & \\
\hline PEOU1 & -.057 & .125 & -.338 & $.849^{\mathrm{a}}$ & & & & & & & \\
\hline PEOU2 & .211 & -.067 & .072 & -.303 & $.842^{\mathrm{a}}$ & & & & & & \\
\hline PEOU3 & .075 & -.192 & .082 & -.226 & -.116 & $.894^{\mathrm{a}}$ & & & & & \\
\hline ATT2 & .294 & -.356 & .236 & .128 & -.201 & .189 & $.739^{\mathrm{a}}$ & & & & \\
\hline ATT3 & -.409 & .208 & -.152 & -.173 & -.244 & -.203 & -.575 & $.781^{\mathrm{a}}$ & & & \\
\hline BI2 & -.069 & -.070 & -.114 & .102 & .084 & -.155 & -.017 & -.354 & $.898^{\mathrm{a}}$ & & \\
\hline BI3 & -.072 & .209 & .035 & -.294 & .139 & .110 & -.183 & -.029 & -.192 & $.831^{\mathrm{a}}$ & \\
\hline BI4 & -.084 & -.021 & -.111 & .008 & -.041 & -.269 & -.135 & .366 & -.400 & -.261 & $.794^{\mathrm{a}}$ \\
\hline
\end{tabular}

a. Measures of Sampling Adequacy (MSA)

Table D3: Survey III, Original TAM PCA: KMO and Bartlett's Test

Kaiser-Meyer-Olkin Measure of Sampling Adequacy.

.822

Bartlett's Test of Sphericity Approx. Chi-Square

273.075

df

Sig.

.000 
Table D4: $\quad$ Survey III, Original TAM PCA: Communalities

\begin{tabular}{lrr}
\hline & Initial & Extraction \\
\hline PU1 & 1.000 & .778 \\
PU2 & 1.000 & .854 \\
PU3 & 1.000 & .748 \\
PEOU1 & 1.000 & .828 \\
PEOU2 & 1.000 & .809 \\
PEOU3 & 1.000 & .656 \\
ATT2 & 1.000 & .890 \\
ATT3 & 1.000 & .862 \\
BI2 & 1.000 & .805 \\
BI3 & 1.000 & .813 \\
BI4 & 1.000 & .729 \\
\hline
\end{tabular}

Extraction Method: Principal

Component Analysis. 
Table D5: $\quad$ Survey III, Original TAM PCA: Total Variance Explained

\begin{tabular}{|c|c|c|c|c|c|c|c|}
\hline \multirow[b]{2}{*}{$\begin{array}{l}\text { Comp } \\
\text { onent }\end{array}$} & \multicolumn{3}{|c|}{ Initial Eigenvalues } & \multicolumn{3}{|c|}{$\begin{array}{c}\text { Extraction Sums of Squared } \\
\text { Loadings }\end{array}$} & \multirow{2}{*}{$\begin{array}{c}\text { Rotation } \\
\text { Sums of } \\
\text { Squared } \\
\text { Loadings a } \\
\\
\text { Total }\end{array}$} \\
\hline & Total & $\begin{array}{c}\% \text { of } \\
\text { Variance }\end{array}$ & $\begin{array}{c}\text { Cumulative } \\
\%\end{array}$ & Total & $\begin{array}{c}\% \text { of } \\
\text { Variance }\end{array}$ & $\begin{array}{c}\text { Cumulative } \\
\%\end{array}$ & \\
\hline 1 & 5.762 & 52.380 & 52.380 & 5.762 & 52.380 & 52.380 & 4.153 \\
\hline 2 & 1.246 & 11.326 & 63.706 & 1.246 & 11.326 & 63.706 & 2.335 \\
\hline 3 & .988 & 8.981 & 72.687 & .988 & 8.981 & 72.687 & 3.243 \\
\hline 4 & .776 & 7.055 & 79.743 & .776 & 7.055 & 79.743 & 3.473 \\
\hline 5 & .631 & 5.732 & 85.475 & & & & \\
\hline 6 & .436 & 3.963 & 89.438 & & & & \\
\hline 7 & .335 & 3.044 & 92.482 & & & & \\
\hline 8 & .286 & 2.597 & 95.079 & & & & \\
\hline 9 & .258 & 2.345 & 97.424 & & & & \\
\hline 10 & .182 & 1.657 & 99.081 & & & & \\
\hline 11 & .101 & .919 & 100.000 & & & & \\
\hline
\end{tabular}

Extraction Method: Principal Component Analysis.

a. When components are correlated, sums of squared loadings cannot be added to obtain a total variance. 
Table D6: Survey III, Original TAM PCA: Structure Matrix

\begin{tabular}{lcccc}
\hline \multicolumn{5}{c}{ Component } \\
& 1 & 2 & 3 & 4 \\
\hline PU2 & .911 & & & \\
PU1 & .873 & & & \\
PU3 & .789 & & & \\
ATT2 & & .905 & & \\
ATT3 & & .735 & & \\
BI3 & & & .867 & \\
BI4 & & & .816 & \\
BI2 & & & & .733 \\
PEOU1 & .714 & & & .764 \\
PEOU2 & & .654 & & \\
PEOU3 & & & & \\
\hline
\end{tabular}

Extraction Method: Principal Component Analysis.

Rotation Method: Oblimin with Kaiser Normalization.

Suppressed coefficients $<.65$ for readability.

Table D7: Survey III, Original TAM PCA: Component Correlation Matrix

\begin{tabular}{lrrrr}
\hline Component & \multicolumn{1}{l}{1} & \multicolumn{1}{l}{2} & 3 & \multicolumn{1}{l}{4} \\
\hline 1 & 1.000 & & & \\
2 & .183 & 1.000 & & \\
3 & .407 & .192 & 1.000 & \\
4 & .392 & .320 & .345 & 1.000 \\
\hline
\end{tabular}

Extraction Method: Principal Component Analysis.

Rotation Method: Oblimin with Kaiser

Normalization. 
Simplified TAM: Survey III PCA

Table D8: Survey III, Simplified TAM PCA: Correlation Matrix ${ }^{\text {a }}$

\begin{tabular}{lrrrrrrrrrrr}
\hline & & \multicolumn{1}{c}{ PEOU } & PEOU & PEOU & & & \\
& PU1 & PU2 & PU3 & PU4 & 1 & 2 & 3 & BI1 & BI2 & BI3 & BI4 \\
\hline PU1 & 1.000 & & & & & & & & & & \\
PU2 & .738 & 1.000 & & & & & & & & & \\
PU3 & .590 & .659 & 1.000 & & & & & & & & \\
PU4 & .592 & .581 & .781 & 1.000 & & & & & & & \\
PEOU1 & .441 & .375 & .563 & .522 & 1.000 & & & & & & \\
PEOU2 & .211 & .294 & .269 & .149 & .518 & 1.000 & & & & & \\
PEOU3 & .495 & .530 & .495 & .347 & .561 & .433 & 1.000 & & & & \\
BI1 & .456 & .428 & .367 & .336 & .497 & .600 & .491 & 1.000 & & & \\
BI2 & .633 & .600 & .580 & .428 & .506 & .364 & .627 & .788 & 1.000 & & \\
BI3 & .335 & .208 & .300 & .370 & .471 & .222 & .324 & .585 & .537 & 1.000 & \\
BI4 & .423 & .441 & .442 & .364 & .356 & .190 & .520 & .587 & .636 & .479 & 1.000 \\
\hline
\end{tabular}

a. Determinant $=.000$

Table D9: Survey III, Simplified TAM PCA: KMO and Bartlett's Test

\begin{tabular}{llr}
\hline Kaiser-Meyer-Olkin Measure of Sampling Adequacy. & .800 \\
Bartlett's Test of & Approx. Chi-Square & 287.591 \\
Sphericity & df & 55 \\
& Sig. & .000 \\
\hline
\end{tabular}


Table D10: Survey III, Simplified TAM PCA: Communalities

\begin{tabular}{lrr}
\hline & Initial & Extraction \\
\hline PU1 & 1.000 & .702 \\
PU2 & 1.000 & .727 \\
PU3 & 1.000 & .798 \\
PU4 & 1.000 & .732 \\
PEOU1 & 1.000 & .652 \\
PEOU2 & 1.000 & .887 \\
PEOU3 & 1.000 & .585 \\
BI1 & 1.000 & .829 \\
BI2 & 1.000 & .801 \\
BI3 & 1.000 & .663 \\
BI4 & 1.000 & .703 \\
\hline
\end{tabular}

Extraction Method: Principal Component Analysis.

Table D11: Survey III, Simplified TAM PCA: Total Variance Explained

\begin{tabular}{|c|c|c|c|c|c|c|c|}
\hline \multirow[b]{2}{*}{$\begin{array}{l}\text { Compo } \\
\text { nent }\end{array}$} & \multicolumn{3}{|c|}{ Initial Eigenvalues } & \multicolumn{3}{|c|}{$\begin{array}{c}\text { Extraction Sums of Squared } \\
\text { Loadings }\end{array}$} & \multirow{2}{*}{$\begin{array}{c}\text { Rotation } \\
\text { Sums of } \\
\text { Squared } \\
\text { Loadings a } \\
\text { Total } \\
\end{array}$} \\
\hline & Total & $\begin{array}{c}\% \text { of } \\
\text { Variance }\end{array}$ & $\begin{array}{c}\text { Cumulative } \\
\% \\
\end{array}$ & Total & $\begin{array}{c}\% \text { of } \\
\text { Variance }\end{array}$ & $\begin{array}{c}\text { Cumulative } \\
\% \\
\end{array}$ & \\
\hline 1 & 5.739 & 52.170 & 52.170 & 5.739 & 52.170 & 52.170 & 4.630 \\
\hline 2 & 1.366 & 12.421 & 64.592 & 1.366 & 12.421 & 64.592 & 4.157 \\
\hline 3 & .974 & 8.853 & 73.445 & .974 & 8.853 & 73.445 & 2.930 \\
\hline 4 & .822 & 7.473 & 80.919 & & & & \\
\hline 5 & .572 & 5.198 & 86.117 & & & & \\
\hline 6 & .456 & 4.149 & 90.267 & & & & \\
\hline 7 & .303 & 2.756 & 93.023 & & & & \\
\hline 8 & .284 & 2.579 & 95.602 & & & & \\
\hline 9 & .218 & 1.985 & 97.587 & & & & \\
\hline 10 & .182 & 1.655 & 99.242 & & & & \\
\hline 11 & .083 & .758 & 100.000 & & & & \\
\hline
\end{tabular}

Extraction Method: Principal Component Analysis.

a. When components are correlated, sums of squared loadings cannot be added to obtain a total variance. 
Table D12: Survey III, Simplified TAM PCA: Structure Matrix

\begin{tabular}{lccc}
\hline \multicolumn{4}{c}{ Component } \\
& 1 & 2 & 3 \\
\hline PU3 & .891 & & \\
PU4 & .851 & & \\
PU2 & .851 & & \\
PU1 & .828 & & \\
BI2 & .654 & .844 & \\
BI1 & & .835 & .657 \\
BI4 & & .822 & \\
BI3 & & .808 & \\
PEOU2 & & & .937 \\
PEOU1 & & & .718 \\
PEOU3 & & & .616 \\
\hline
\end{tabular}

Extraction Method: Principal

Component Analysis.

Rotation Method: Oblimin with Kaiser

Normalization.

Suppressed coefficients $<.65$ for readability.

Table D13: Survey III, Simplified TAM PCA: Component Correlation Matrix

\begin{tabular}{lrrr}
\hline Component & \multicolumn{1}{c}{1} & \multicolumn{1}{l}{2} & 3 \\
\hline 1 & 1.000 & & \\
2 & .495 & 1.000 & \\
3 & .338 & .392 & 1.000 \\
\hline
\end{tabular}

Extraction Method: Principal Component

Analysis.

Rotation Method: Oblimin with Kaiser

Normalization. 
APPENDIX E

Path Analysis Tables: Survey I 
Original TAM: Survey I Path Analysis

Table E1: Survey I, Original TAM PA: PEOU and PU Correlations

\begin{tabular}{llrr}
\hline & & PU & \multicolumn{2}{c}{ PEOU } \\
\hline PU & Pearson Correlation & 1 & $.566^{* *}$ \\
& Sig. (2-tailed) & & .000 \\
& $\mathrm{~N}$ & 51 & 51 \\
\hline PEOU & Pearson Correlation & $.566^{* * *}$ & 1 \\
& Sig. (2-tailed) & .000 & \\
& $\mathrm{~N}$ & 51 & 51 \\
\hline
\end{tabular}

**. Correlation is significant at the 0.01 level (2-tailed).

Table E2: $\quad$ Survey I, Original TAM PA: PEOU $\rightarrow$ PU Model Summary

\begin{tabular}{llrrr}
\hline Model & $\mathrm{R}$ & R Square & $\begin{array}{c}\text { Adjusted } \mathrm{R} \\
\text { Square }\end{array}$ & $\begin{array}{c}\text { Std. Error of the } \\
\text { Estimate }\end{array}$ \\
\hline 1 & $.566^{\mathrm{a}}$ & .320 & .306 & .83294885 \\
\hline
\end{tabular}

a. Predictors: (Constant), PEOU

Table E3: $\quad$ Survey I, Original TAM PA: PEOU $\rightarrow$ PU Coefficients a

\begin{tabular}{rrrrrrr}
\hline & & \multicolumn{2}{c}{$\begin{array}{c}\text { Unstandardized } \\
\text { Coefficients }\end{array}$} & \multicolumn{2}{c}{$\begin{array}{c}\text { Standardized } \\
\text { Coefficients }\end{array}$} & \\
\multicolumn{1}{c}{ Model } & \multicolumn{1}{c}{ B } & Std. Error & Beta & \multicolumn{1}{c}{ t } & \multicolumn{1}{c}{ Sig. } \\
\hline 1 & (Constant) & $1.185 \mathrm{E}-16$ & .117 & & .000 & 1.000 \\
& PEOU & .566 & .118 & .566 & 4.803 & .000 \\
\hline
\end{tabular}

a. Dependent Variable: PU 
Table E4: $\quad$ Survey I, Original TAM PA: PU, PEOU, and ATT Correlations

\begin{tabular}{llrrr}
\hline & & PU & PEOU & ATT \\
\hline PU & Pearson Correlation & 1 & $.566^{* *}$ & $.478^{* *}$ \\
& Sig. (2-tailed) & & .000 & .000 \\
& $\mathrm{~N}$ & 51 & 51 & 51 \\
\hline PEOU Pearson Correlation & $.566^{* *}$ & 1 & $.498^{* *}$ \\
& Sig. (2-tailed) & .000 & & .000 \\
& $\mathrm{~N}$ & 51 & 51 & 51 \\
\hline ATT & Pearson Correlation & $.478^{* *}$ & $.498^{* *}$ & 1 \\
& Sig. (2-tailed) & .000 & .000 & \\
& $\mathrm{~N}$ & 51 & 51 & 51 \\
\hline **. Correlation is significant at the 0.01 level (2-tailed). &
\end{tabular}

Table E5: $\quad$ Survey I, Original TAM PA: PEOU and PU $\rightarrow$ ATT Model Summary

\begin{tabular}{llrrr}
\hline Model & $\mathrm{R}$ & $\begin{array}{c}\mathrm{R} \\
\text { Square }\end{array}$ & $\begin{array}{c}\text { Adjusted R } \\
\text { Square }\end{array}$ & $\begin{array}{c}\text { Std. Error of the } \\
\text { Estimate }\end{array}$ \\
\hline 1 & $.552^{\mathrm{a}}$ & .305 & .276 & .85115675 \\
\hline
\end{tabular}

a. Predictors: (Constant), PEOU, PU

Table E6: $\quad$ Survey I, Original TAM PA: PU and ATT $\rightarrow$ BI Coefficients a

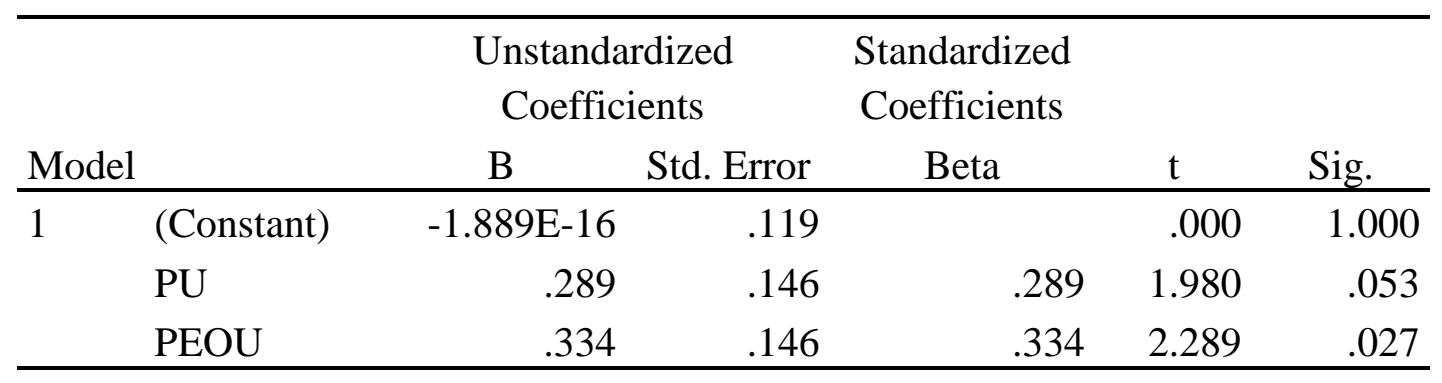

a. Dependent Variable: ATT 
Table E7: $\quad$ Survey I, Original TAM PA: PU, ATT, and BI Correlations

\begin{tabular}{llrrr}
\hline & & PU & ATT & BI \\
\hline PU & Pearson Correlation & 1 & $.478^{* *}$ & $.455^{* *}$ \\
& Sig. (2-tailed) & & .000 & .001 \\
& $\mathrm{~N}$ & 51 & 51 & 51 \\
\hline ATT & Pearson Correlation & $.478^{* *}$ & 1 & $.484^{* *}$ \\
& Sig. (2-tailed) & .000 & & .000 \\
& N & 51 & 51 & 51 \\
\hline BI & Pearson Correlation & $.455^{* *}$ & $.484^{* *}$ & 1 \\
& Sig. (2-tailed) & .001 & .000 & \\
& N & 51 & 51 & 51 \\
\hline$* *$ Correlation is significant at the 0.01 level (2-tailed). &
\end{tabular}

Table E8: $\quad$ Survey I, Original TAM PA: PU and ATT $\rightarrow$ BI Model Summary

\begin{tabular}{lrrrr}
\hline Model & R & R Square & Adjusted R Square & Std. Error of the Estimate \\
\hline 1 & $.546^{\mathrm{a}}$ & .298 & .269 & .85484657 \\
\hline
\end{tabular}

a. Predictors: (Constant), ATT, PU

Table E9: $\quad$ Survey I, Original TAM PA: PU and ATT $\rightarrow$ BI Coefficients ${ }^{\text {a }}$

\begin{tabular}{rlrrrrr}
\hline & \multicolumn{2}{c}{$\begin{array}{c}\text { Unstandardized } \\
\text { Coefficients }\end{array}$} & \multicolumn{2}{c}{ Standardized } \\
Coefficients & & \\
Model & \multicolumn{2}{c}{ B } & Std. Error & Beta & \multicolumn{1}{c}{ t } & \multicolumn{1}{c}{ Sig. } \\
\hline 1 & (Constant) & $1.527 \mathrm{E}-16$ & .120 & & .000 & 1.000 \\
& PU & .290 & .138 & .290 & 2.104 & .041 \\
& ATT & .345 & .138 & .345 & 2.507 & .016 \\
\hline
\end{tabular}

a. Dependent Variable: BI 
Simplified TAM: Survey I Path Analysis

Table E10: Survey I, Simplified TAM PA: PU, PEOU, and BI Correlations

\begin{tabular}{llrrr}
\hline & & PU & BI & \multicolumn{2}{c}{ PEOU } \\
\hline PU & Pearson Correlation & 1 & $.544^{* * *}$ & $.669^{* *}$ \\
& Sig. (2-tailed) & & .000 & .000 \\
& $\mathrm{~N}$ & 51 & 51 & 51 \\
\hline BI & Pearson Correlation & $.544^{* *}$ & 1 & $.385^{* *}$ \\
& Sig. (2-tailed) & .000 & & .005 \\
& $\mathrm{~N}$ & 51 & 51 & 51 \\
\hline PEOU & Pearson Correlation & $.669^{* *}$ & $.385^{* *}$ & 1 \\
& Sig. (2-tailed) & .000 & .005 & \\
& $\mathrm{~N}$ & 51 & 51 & 51 \\
\hline
\end{tabular}

**. Correlation is significant at the 0.01 level (2-tailed).

Table E11: $\quad$ Survey I, Simplified TAM PA: PEOU $\rightarrow$ PU Model Summary

\begin{tabular}{lrrrr}
\hline Model & R & R Square & Adjusted R Square & Std. Error of the Estimate \\
\hline 1 & $.669^{\mathrm{a}}$ & .448 & .437 & .75051619 \\
\hline
\end{tabular}

a. Predictors: (Constant), PEOU

Table E12: $\quad$ urvey I, Simplified TAM PA: PEOU $\rightarrow$ PU Coefficients ${ }^{\text {a }}$

\begin{tabular}{|c|c|c|c|c|c|c|}
\hline \multirow{2}{*}{\multicolumn{2}{|c|}{ Model }} & \multicolumn{2}{|c|}{$\begin{array}{c}\text { Unstandardized } \\
\text { Coefficients }\end{array}$} & \multirow{2}{*}{$\begin{array}{c}\text { Standardized } \\
\text { Coefficients } \\
\text { Beta } \\
\end{array}$} & \multirow[b]{2}{*}{$\mathrm{t}$} & \multirow[b]{2}{*}{ Sig. } \\
\hline & & $\mathrm{B}$ & Std. Error & & & \\
\hline \multirow[t]{2}{*}{1} & (Constant) & $8.290 \mathrm{E}-17$ & .105 & & .000 & 1.000 \\
\hline & PEOU & .669 & .106 & .669 & 6.306 & .000 \\
\hline
\end{tabular}

a. Dependent Variable: PU 
Table E13: Survey I, Simplified TAM PA: PU and PEOU $\rightarrow$ BI Model Summary

\begin{tabular}{llrrr}
\hline Model & R & R Square & $\begin{array}{c}\text { Adjusted R } \\
\text { Square }\end{array}$ & $\begin{array}{c}\text { Std. Error of the } \\
\text { Estimate }\end{array}$ \\
\hline 1 & $.545^{\mathrm{a}}$ & .297 & .268 & .85581006 \\
\hline
\end{tabular}

a. Predictors: (Constant), PU, PEOU

Table E14: Survey I, Simplified TAM PA: PU and PEOU $\rightarrow$ BI Coefficients ${ }^{\text {a }}$

\begin{tabular}{|c|c|c|c|c|c|c|}
\hline \multirow{2}{*}{\multicolumn{2}{|c|}{ Model }} & \multicolumn{2}{|c|}{$\begin{array}{c}\text { Unstandardized } \\
\text { Coefficients }\end{array}$} & \multirow{2}{*}{$\begin{array}{c}\text { Standardized } \\
\text { Coefficients } \\
\text { Beta } \\
\end{array}$} & \multirow[b]{2}{*}{$\mathrm{t}$} & \multirow[b]{2}{*}{ Sig. } \\
\hline & & $\mathrm{B}$ & Std. Error & & & \\
\hline \multirow[t]{3}{*}{1} & (Constant) & $9.092 \mathrm{E}-17$ & .120 & & .000 & 1.000 \\
\hline & PEOU & .037 & .163 & .037 & .228 & .821 \\
\hline & $\mathrm{PU}$ & .519 & .163 & .519 & 3.188 & .003 \\
\hline
\end{tabular}

a. Dependent Variable: BI 
APPENDIX F

Path Analysis Tables: Survey II 
Original TAM: Survey II Path Analysis

Table F1: $\quad$ Survey II, Original TAM PA: PEOU and PU Correlations

\begin{tabular}{llrr}
\hline & & PU & PEOU \\
\hline PU & Pearson Correlation & 1 & $.341^{* *}$ \\
& Sig. (2-tailed) & & .002 \\
& $\mathrm{~N}$ & 80 & 80 \\
\hline PEOU & Pearson Correlation & $.341^{* *}$ & 1 \\
& Sig. (2-tailed) & .002 & 80 \\
& $\mathrm{~N}$ & 80 & \\
& & & \\
\end{tabular}

**. Correlation is significant at the 0.01 level (2-tailed).

Table F2: $\quad$ Survey II, Original TAM PA: PEOU $\rightarrow$ PU Model Summary

\begin{tabular}{lcccc}
\hline & & \multicolumn{2}{c}{$\begin{array}{c}\text { Adjusted R } \\
\text { Square }\end{array}$} & $\begin{array}{c}\text { Std. Error of the } \\
\text { Estimate }\end{array}$ \\
\hline 1 & $\mathrm{R}$ & $\mathrm{R}$ Square & \multicolumn{2}{c}{ Squal } \\
\hline a. Predictors: (Constant), PEOU
\end{tabular}

Table F3: $\quad$ Survey II, Original TAM PA: PEOU $\rightarrow$ PU Coefficients ${ }^{\text {a }}$

\begin{tabular}{rlrrrrr}
\hline & \multicolumn{2}{c}{ Unstandardized } & \multicolumn{2}{c}{ Standardized } \\
Coefficients & \multicolumn{2}{c}{ Coefficients } & & \\
\multicolumn{1}{c}{ Model } & \multicolumn{2}{c}{ B } & Std. Error & Beta & \multicolumn{1}{c}{ t } & \multicolumn{1}{c}{ Sig. } \\
\hline 1 & (Constant) & $2.647 \mathrm{E}-17$ & .106 & & .000 & 1.000 \\
& PEOU & .341 & .106 & .341 & 3.205 & .002 \\
\hline
\end{tabular}

a. Dependent Variable: PU

Table F4: $\quad$ Survey II, Original TAM PA: PU, PEOU, and ATT Correlations

\begin{tabular}{llrrr}
\hline & & PU & PEOU & \multicolumn{1}{c}{ ATT } \\
\hline PU & Pearson Correlation & 1 & $.341^{* *}$ & $.466^{* *}$ \\
& Sig. (2-tailed) & & .002 & .000 \\
& $\mathrm{~N}$ & 80 & 80 & 80 \\
\hline PEOU & Pearson Correlation & $.341^{* *}$ & 1 & $.223^{*}$
\end{tabular}




\begin{tabular}{llrrr} 
& Sig. (2-tailed) & .002 & & .046 \\
& $\mathrm{~N}$ & 80 & 80 & 80 \\
\hline \multirow{2}{*}{ ATT } & Pearson Correlation & $.466^{* *}$ & $.223^{*}$ & 1 \\
& Sig. (2-tailed) & .000 & .046 & \\
& $\mathrm{~N}$ & 80 & 80 & 80 \\
\hline
\end{tabular}

**. Correlation is significant at the 0.01 level (2-tailed).

*. Correlation is significant at the 0.05 level (2-tailed).

Table F5: $\quad$ Survey II, Original TAM PA: PEOU and PU $\rightarrow$ ATT Model Summary

\begin{tabular}{llrrr}
\hline Model & R & R Square & $\begin{array}{c}\text { Adjusted R } \\
\text { Square }\end{array}$ & $\begin{array}{l}\text { Std. Error of } \\
\text { the Estimate }\end{array}$ \\
\hline 1 & $.471^{\mathrm{a}}$ & .221 & .201 & .89374642 \\
\hline
\end{tabular}

a. Predictors: (Constant), PEOU, PU

Table F6: $\quad$ Survey II, Original TAM PA: PEOU and PU $\rightarrow$ ATT Coefficients ${ }^{\text {a }}$

\begin{tabular}{rlrrrrr}
\hline & \multicolumn{2}{c}{$\begin{array}{c}\text { Unstandardized } \\
\text { Coefficients }\end{array}$} & \multicolumn{2}{c}{ Standardized } \\
Coefficients & & \\
Model & \multicolumn{2}{c}{ B } & Std. Error & Beta & \multicolumn{1}{c}{ t } & \multicolumn{1}{c}{ Sig. } \\
\hline 1 & (Constant) & $-1.814 \mathrm{E}-16$ & .100 & & .000 & 1.000 \\
& PU & .441 & .107 & .441 & 4.119 & .000 \\
& PEOU & .073 & .107 & .073 & .683 & .496 \\
\hline
\end{tabular}

a. Dependent Variable: ATT

Table F7: $\quad$ Survey II, Original TAM PA: PU, ATT, and BI Correlations

\begin{tabular}{llrrr}
\hline & & PU & \multicolumn{1}{c}{ BI } & \multicolumn{1}{c}{ ATT } \\
\hline PU & Pearson Correlation & 1 & $.383^{* *}$ & $.466^{* *}$ \\
& Sig. (2-tailed) & & .000 & .000 \\
& $\mathrm{~N}$ & 80 & 80 & 80 \\
\hline BI & Pearson Correlation & $.383^{* *}$ & 1 & $.409^{* *}$
\end{tabular}




\begin{tabular}{llrrr} 
& Sig. (2-tailed) & .000 & & .000 \\
& $\mathrm{~N}$ & 80 & 80 & 80 \\
\hline \multirow{2}{*}{ ATT } & Pearson Correlation & $.466^{* *}$ & $.409^{* *}$ & 1 \\
& Sig. (2-tailed) & .000 & .000 & \\
& $\mathrm{~N}$ & 80 & 80 & 80 \\
\hline
\end{tabular}

**. Correlation is significant at the 0.01 level (2-tailed).

Table F8: $\quad$ Survey II, Original TAM PA: PU and ATT $\rightarrow$ BI Model Summary

\begin{tabular}{|c|c|c|c|c|}
\hline Model & $\mathrm{R}$ & R Square & $\begin{array}{l}\text { Adjusted R } \\
\text { Square }\end{array}$ & $\begin{array}{l}\text { Std. Error of the } \\
\text { Estimate }\end{array}$ \\
\hline 1 & $.464^{\mathrm{a}}$ & .215 & .195 & .89741279 \\
\hline
\end{tabular}

a. Predictors: (Constant), ATT, PU

Table F9: $\quad$ Survey II, Original TAM PA: PU and ATT $\rightarrow$ BI Coefficients ${ }^{\text {a }}$

\begin{tabular}{rlrrrrr}
\hline & \multicolumn{2}{c}{$\begin{array}{c}\text { Unstandardized } \\
\text { Coefficients }\end{array}$} & \multicolumn{2}{c}{$\begin{array}{c}\text { Standardized } \\
\text { Coefficients }\end{array}$} \\
Model & \multicolumn{2}{c}{ B } & Std. Error & Beta & \multicolumn{1}{c}{ t } & \multicolumn{1}{c}{ Sig. } \\
\hline 1 & (Constant) & $2.007 \mathrm{E}-16$ & .100 & & .000 & 1.000 \\
& PU & .246 & .114 & .246 & 2.159 & .034 \\
& ATT & .295 & .114 & .295 & 2.583 & .012 \\
\hline
\end{tabular}

a. Dependent Variable: BI 
Simplified TAM: Survey II Path Analysis

Table F10: Survey II, Simplified TAM PA: PU, PEOU, and BI Correlations

\begin{tabular}{|c|c|c|c|c|}
\hline & & $\mathrm{PU}$ & PEOU & BI \\
\hline \multirow[t]{3}{*}{$\mathrm{PU}$} & Pearson Correlation & 1 & $.389^{* * *}$ & $.379^{* *}$ \\
\hline & Sig. (2-tailed) & & .000 & .001 \\
\hline & $\mathrm{N}$ & 80 & 80 & 80 \\
\hline \multirow[t]{3}{*}{ PEOU } & Pearson Correlation & $.389^{* * *}$ & 1 & .187 \\
\hline & Sig. (2-tailed) & .000 & & .098 \\
\hline & $\mathrm{N}$ & 80 & 80 & 80 \\
\hline \multirow[t]{3}{*}{$\mathrm{BI}$} & Pearson Correlation & $.379^{* *}$ & .187 & 1 \\
\hline & Sig. (2-tailed) & .001 & .098 & \\
\hline & $\mathrm{N}$ & 80 & 80 & 80 \\
\hline
\end{tabular}

**. Correlation is significant at the 0.01 level (2-tailed).

Table F11: $\quad$ Survey II, Simplified TAM PA: PEOU $\rightarrow$ PU Model Summary

\begin{tabular}{llrrr}
\hline Model & R & R Square & Adjusted R Square & Std. Error of the Estimate \\
\hline 1 & $.389^{\mathrm{a}}$ & .152 & .141 & .92697922 \\
\hline
\end{tabular}

a. Predictors: (Constant), PEOU

Table F12: $\quad$ Survey II, Simplified TAM PA: PEOU $\rightarrow$ PU Coefficients ${ }^{\text {a }}$

\begin{tabular}{rlrrrrr}
\hline & \multicolumn{2}{c}{$\begin{array}{l}\text { Unstandardized } \\
\text { Coefficients }\end{array}$} & \multicolumn{2}{c}{$\begin{array}{c}\text { Standardized } \\
\text { Coefficients }\end{array}$} & \\
\multicolumn{1}{c}{ Model } & \multicolumn{1}{c}{ B } & Std. Error & Beta & \multicolumn{1}{c}{ t } & \multicolumn{1}{c}{ Sig. } \\
\hline 1 & (Constant) & $4.225 \mathrm{E}-18$ & .104 & & .000 & 1.000 \\
& PEOU & .389 & .104 & .389 & 3.733 & .000 \\
\hline
\end{tabular}

a. Dependent Variable: PU 
Table F13 Survey II, Simplified TAM PA: PU and PEOU $\rightarrow$ BI Model Summary

\begin{tabular}{llrrr}
\hline Model & R & R Square & $\begin{array}{c}\text { Adjusted R } \\
\text { Square }\end{array}$ & $\begin{array}{l}\text { Std. Error of } \\
\text { the Estimate }\end{array}$ \\
\hline 1 & $.381^{\mathrm{a}}$ & .145 & .123 & .93642532 \\
\hline
\end{tabular}

a. Predictors: (Constant), PEOU, PU

Table F14: Survey II, Simplified TAM PA: PU and PEOU $\rightarrow$ BI Coefficients a

\begin{tabular}{rlrrrrr}
\hline & & \multicolumn{2}{c}{$\begin{array}{l}\text { Unstandardized } \\
\text { Coefficients }\end{array}$} & \multicolumn{2}{c}{$\begin{array}{c}\text { Standardized } \\
\text { Coefficients }\end{array}$} \\
Model & \multicolumn{1}{c}{ B } & Std. Error & Beta & \multicolumn{1}{c}{ t } & \multicolumn{1}{c}{ Sig. } \\
\hline 1 & (Constant) & $1.396 \mathrm{E}-16$ & .105 & & .000 & 1.000 \\
& PU & .361 & .114 & .361 & 3.155 & .002 \\
& PEOU & .046 & .114 & .046 & .402 & .689 \\
\hline
\end{tabular}

a. Dependent Variable: BI 
APPENDIX G

\section{Path Analysis Tables: Survey III}


Original TAM: Survey III Path Analysis

Table G1: $\quad$ Survey III, Original TAM PA: PEOU and PU Correlations

\begin{tabular}{llrr}
\hline & \multicolumn{2}{c}{ PU } & $.392^{* *}$ \\
\hline PU & Pearson Correlation & 1 & .009 \\
& Sig. (2-tailed) & & 43 \\
\hline $\mathrm{N}$ & 43 & 1 \\
\hline PEOU & Pearson Correlation & $.392^{* *}$ & \\
& Sig. (2-tailed) & .009 & 43 \\
& $\mathrm{~N}$ & 43 & \\
\hline
\end{tabular}

**. Correlation is significant at the 0.01 level (2-tailed).

Table G2: $\quad$ Survey III, Original TAM PA: PEOU $\rightarrow$ PU Model Summary

\begin{tabular}{|c|c|c|c|c|}
\hline Model & $\mathrm{R}$ & R Square & $\begin{array}{c}\text { Adjusted R } \\
\text { Square }\end{array}$ & $\begin{array}{l}\text { Std. Error of the } \\
\text { Estimate }\end{array}$ \\
\hline$\overline{1}$ & $.392^{\mathrm{a}}$ & .154 & .133 & .93099029 \\
\hline
\end{tabular}

a. Predictors: (Constant), PEOU

Table G3: $\quad$ Survey III, Original TAM PA: PEOU $\rightarrow$ PU Coefficients ${ }^{\text {a }}$

\begin{tabular}{|c|c|c|c|c|c|c|}
\hline \multirow[b]{2}{*}{ Model } & & \multicolumn{2}{|c|}{$\begin{array}{c}\text { Unstandardized } \\
\text { Coefficients }\end{array}$} & \multirow{2}{*}{$\begin{array}{c}\text { Standardized } \\
\text { Coefficients } \\
\text { Beta }\end{array}$} & \multirow[b]{2}{*}{$\mathrm{t}$} & \multirow[b]{2}{*}{ Sig. } \\
\hline & & $\mathrm{B}$ & Std. Error & & & \\
\hline \multirow[t]{2}{*}{1} & (Constant) & $2.078 \mathrm{E}-16$ & .142 & & .000 & 1.000 \\
\hline & PEOU & .392 & .144 & .392 & 2.731 & .009 \\
\hline
\end{tabular}

a. Dependent Variable: PU 
Table G4: $\quad$ Survey III, Original TAM PA: PU, PEOU, and ATT Correlations

\begin{tabular}{|c|c|c|c|c|}
\hline & & $\mathrm{PU}$ & PEOU & ATT \\
\hline \multirow[t]{3}{*}{$\mathrm{PU}$} & Pearson Correlation & 1 & $.392^{* * *}$ & .183 \\
\hline & Sig. (2-tailed) & & .009 & .240 \\
\hline & $\mathrm{N}$ & 43 & 43 & 43 \\
\hline \multirow[t]{3}{*}{ PEOU } & Pearson Correlation & $.392^{* * *}$ & 1 & $.320^{*}$ \\
\hline & Sig. (2-tailed) & .009 & & .036 \\
\hline & $\mathrm{N}$ & 43 & 43 & 43 \\
\hline \multirow[t]{3}{*}{$\overline{\text { ATT }}$} & Pearson Correlation & .183 & $.320^{*}$ & 1 \\
\hline & Sig. (2-tailed) & .240 & .036 & \\
\hline & $\mathrm{N}$ & 43 & 43 & 43 \\
\hline
\end{tabular}

**. Correlation is significant at the 0.01 level (2-tailed).

*. Correlation is significant at the 0.05 level (2-tailed).

Table G5: Survey III, Original TAM PA: PEOU and PU ATT Model Summary

\begin{tabular}{lcrrr}
\hline Model & R & R Square & $\begin{array}{c}\text { Adjusted R } \\
\text { Square }\end{array}$ & $\begin{array}{l}\text { Std. Error of } \\
\text { the Estimate }\end{array}$ \\
\hline 1 & $.326^{\mathrm{a}}$ & .106 & .062 & .96861587 \\
\hline
\end{tabular}

a. Predictors: (Constant), PU, PEOU

Table G6: $\quad$ Survey III, Original TAM PA: PEOU and PU $\rightarrow$ ATT Coefficients ${ }^{\text {a }}$

\begin{tabular}{rlrrrrr}
\hline & \multicolumn{2}{c}{ Unstandardized } & \multicolumn{2}{c}{ Standardized } \\
Coefficients & \multicolumn{2}{c}{ Coefficients } & & \\
Model & \multicolumn{1}{c}{ B } & Std. Error & Beta & \multicolumn{1}{c}{ t } & \multicolumn{1}{c}{ Sig. } \\
\hline 1 & (Constant) & $-2.469 E-17$ & .148 & & .000 & 1.000 \\
& PEOU & .294 & .162 & .294 & 1.806 & .078 \\
& PU & .068 & .162 & .068 & .419 & .678 \\
\hline
\end{tabular}

a. Dependent Variable: ATT 
Table G7: $\quad$ Survey III, Original TAM PA: PU, ATT, and BI Correlations

\begin{tabular}{llrrr}
\hline & & \multicolumn{2}{c}{ PUT } & \multicolumn{1}{c}{ BI } \\
\hline PU & Pearson Correlation & 1 & .183 & $.407^{* *}$ \\
& Sig. (2-tailed) & & .240 & .007 \\
& N & 43 & 43 & 43 \\
\hline \multirow{2}{*}{ ATT } & Pearson Correlation & .183 & 1 & .192 \\
& Sig. (2-tailed) & .240 & & .217 \\
& N & 43 & 43 & 43 \\
\hline BI & Pearson Correlation & $.407^{* *}$ & .192 & 1 \\
& Sig. (2-tailed) & .007 & .217 & \\
& N & 43 & 43 & 43 \\
\hline$* *$ Correlation is significant at the 0.01 level (2-tailed). &
\end{tabular}

**. Correlation is significant at the 0.01 level (2-tailed).

Table G8: $\quad$ Survey III, Original TAM PA: PU and ATT $\rightarrow$ BI Model Summary

\begin{tabular}{lrrrr}
\hline Model & R & R Square & $\begin{array}{c}\text { Adjusted R } \\
\text { Square }\end{array}$ & $\begin{array}{l}\text { Std. Error of } \\
\text { the Estimate }\end{array}$ \\
\hline 1 & $.424^{\mathrm{a}}$ & .180 & .139 & .92796699 \\
\hline
\end{tabular}

a. Predictors: (Constant), ATT, PU

Table G9: $\quad$ Survey III, Original TAM PA: PU and ATT $\rightarrow$ BI Coefficients ${ }^{\text {a }}$

\begin{tabular}{rlrrrrr}
\hline & \multicolumn{2}{c}{$\begin{array}{c}\text { Unstandardized } \\
\text { Coefficients }\end{array}$} & \multicolumn{2}{c}{$\begin{array}{c}\text { Standardized } \\
\text { Coefficients }\end{array}$} & & \\
Model & \multicolumn{1}{c}{ B } & Std. Error & Beta & \multicolumn{1}{c}{ t } & \multicolumn{1}{c}{ Sig. } \\
\hline 1 & (Constant) & $-1.311 \mathrm{E}-17$ & .142 & & .000 & 1.000 \\
& PU & .385 & .146 & .385 & 2.641 & .012 \\
& ATT & .122 & .146 & .122 & .834 & .409 \\
\hline
\end{tabular}

a. Dependent Variable: BI 
Simplified TAM: Survey III Path Analysis

Table G10: Survey III, Simplified TAM PA: PU, PEOU, and BI Correlations

\begin{tabular}{|c|c|c|c|c|}
\hline & & $\mathrm{PU}$ & $\mathrm{BI}$ & PEOU \\
\hline \multirow[t]{3}{*}{$\mathrm{PU}$} & Pearson Correlation & 1 & $.495^{* *}$ & .338 \\
\hline & Sig. (2-tailed) & & .001 & .027 \\
\hline & $\mathrm{N}$ & 43 & 43 & 43 \\
\hline \multirow[t]{3}{*}{$\mathrm{BI}$} & Pearson Correlation & $.495^{* *}$ & 1 & $.392^{* *}$ \\
\hline & Sig. (2-tailed) & .001 & & .009 \\
\hline & $\mathrm{N}$ & 43 & 43 & 43 \\
\hline \multirow[t]{3}{*}{$\overline{\mathrm{PEOU}}$} & Pearson Correlation & $.338^{*}$ & $.392^{* * *}$ & 1 \\
\hline & Sig. (2-tailed) & .027 & .009 & \\
\hline & $\mathrm{N}$ & 43 & 43 & 43 \\
\hline
\end{tabular}

**. Correlation is significant at the 0.01 level (2-tailed).

*. Correlation is significant at the 0.05 level (2-tailed).

Table G11: Survey III, Simplified TAM PA: PEOU $\rightarrow$ PU Model Summary

\begin{tabular}{lrrrr}
\hline Model & R & R Square & $\begin{array}{c}\text { Adjusted R } \\
\text { Square }\end{array}$ & $\begin{array}{l}\text { Std. Error of } \\
\text { the Estimate }\end{array}$ \\
\hline 1 & $.338^{\mathrm{a}}$ & .114 & .092 & .95272497 \\
\hline
\end{tabular}

a. Predictors: (Constant), PEOU

Table G12: Survey III, Simplified TAM PA: PEOU $\rightarrow$ PU Coefficients a

\begin{tabular}{|c|c|c|c|c|c|c|}
\hline \multirow{2}{*}{\multicolumn{2}{|c|}{ Model }} & \multicolumn{2}{|c|}{$\begin{array}{l}\text { Unstandardized } \\
\text { Coefficients }\end{array}$} & \multirow{2}{*}{$\begin{array}{c}\text { Standardized } \\
\text { Coefficients } \\
\text { Beta } \\
\end{array}$} & \multirow[b]{2}{*}{ t } & \multirow[b]{2}{*}{ Sig. } \\
\hline & & $\mathrm{B}$ & Std. Error & & & \\
\hline \multirow[t]{2}{*}{1} & (Constant) & $1.213 \mathrm{E}-16$ & .145 & & .000 & 1.000 \\
\hline & PEOU & .338 & .147 & .338 & 2.296 & .027 \\
\hline
\end{tabular}

a. Dependent Variable: PU 
Table G13: Survey III, Simplified TAM PA: PU and PEOU $\rightarrow$ BI Model Summary

\begin{tabular}{llrrr}
\hline Model & R & R Square & $\begin{array}{c}\text { Adjusted R } \\
\text { Square }\end{array}$ & $\begin{array}{c}\text { Std. Error of the } \\
\text { Estimate }\end{array}$ \\
\hline 1 & $.550^{\mathrm{a}}$ & .303 & .268 & .85576635 \\
\hline
\end{tabular}

a. Predictors: (Constant), PU, PEOU

Table G14: Survey III, Simplified TAM PA: PU and PEOU $\rightarrow$ BI Coefficients a

\begin{tabular}{|c|c|c|c|c|c|c|}
\hline \multirow{2}{*}{\multicolumn{2}{|c|}{ Model }} & \multicolumn{2}{|c|}{$\begin{array}{l}\text { Unstandardized } \\
\text { Coefficients }\end{array}$} & \multirow{2}{*}{$\begin{array}{c}\text { Standardized } \\
\text { Coefficients } \\
\text { Beta } \\
\end{array}$} & \multirow[b]{2}{*}{$\mathrm{t}$} & \multirow[b]{2}{*}{ Sig. } \\
\hline & & $\mathrm{B}$ & Std. Error & & & \\
\hline \multirow[t]{3}{*}{1} & (Constant) & $3.528 \mathrm{E}-18$ & .131 & & .000 & 1.000 \\
\hline & PEOU & .254 & .140 & .254 & 1.809 & .078 \\
\hline & PU & .410 & .140 & .410 & 2.921 & .006 \\
\hline
\end{tabular}

a. Dependent Variable: BI 